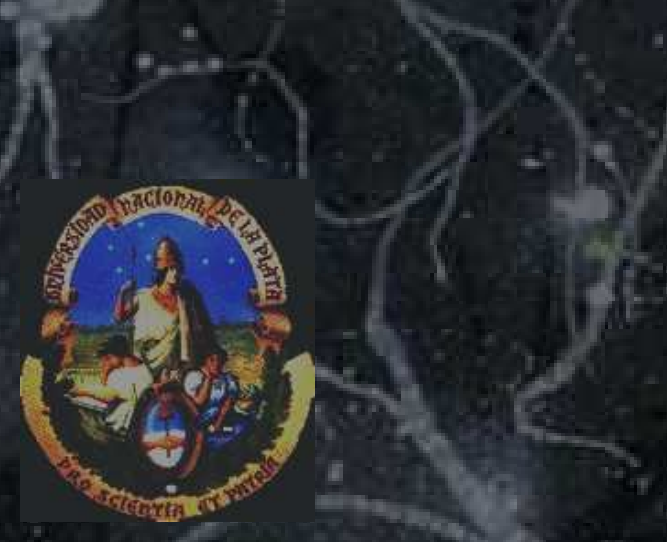

Facultad de Ciencias Astronómicas y Geofísicas Universidad Nacional de La Plata

\title{
Propiedades químicas de galaxias y sus componentes en un universo jerárquico
}

\section{Trabajo de tesis elaborado por el Lic. \\ Ignacio Daniel Gargiulo}

Directora

Dr. Nelson D. Padilla

Dra. Sofía A. Cora

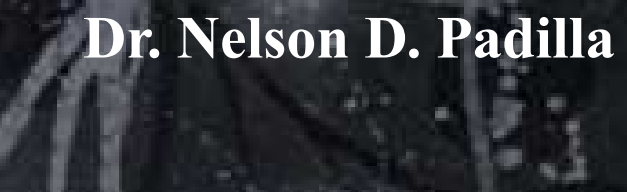





\section{Resumen}

Utilizamos el modelo semianalítico de formación y evolución de galaxias SAG para estudiar las propiedades químicas de las galaxias elípticas y la componente de bulbo de galaxias tipo Vía Láctea en el marco del paradigma cosmológico $\Lambda \mathrm{CDM}$. En primera instancia, se investigó el origen de la correlación observada entre el cociente de abundancias $[\alpha / \mathrm{Fe}]$ y la masa estelar de galaxias elípticas. Esta pendiente es positiva, siendo las galaxias de mayor masa las que contienen una mayor abundancia de elementos $\alpha$ respecto al hierro que las de menor masa, lo cual suele ser señalado como un problema del paradigma jerárquico. Mostramos que mediante la implementación de una nueva función inicial de masa integrada galáctica con exceso en estrellas de alta masa (Top Heavy Integrated Initial Mass Function, TH-IGIMF) dependiente de la tasa de formación estelar (SFR) en el modelo se logra reproducir la tendencia observada, lo cual no ocurre con una función inicial de masa (IMF) universal como la de Salpeter. Las galaxias más masivas alcanzan cocientes $[\alpha / \mathrm{Fe}]$ más altos porque están caracterizadas por pendientes más planas en la IMF como resultado de sus mayores SFR. Estudiar las propiedades químicas de galaxias elípticas tuvo como objetivo secundario profundizar el estudio de los mecanismos de formación de esferoides para llevar adelante la segunda parte del trabajo.

Estudiamos entonces las propiedades químicas de los bulbos de galaxias tipo Vía Láctea (VL) a través de sus poblaciones estelares (PEs) con el objetivo de evaluar la importancia relativa de los diferentes mecanismos de formación de bulbos en este caso en particular. Incluimos las modificaciones necesarias en el modelo SAG para lograr resolver PEs en las galaxias. Luego, promediamos las propiedades de las PEs de las galaxias tipo VL para lograr un mayor rango dinámico. Se siguieron y se compararon dos diferentes criterios para seleccionar galaxias tipo VL, uno basado en las propiedades fotométricas de las galaxias y otro basado en el origen y contexto cosmológico del grupo local de galaxias (LG, por su sigla en inglés) ampliando la perspectiva de nuestro análisis. Comparamos las propiedades químicas de las PEs con estimaciones observacionales de abundancias de estrellas del bulbo. De nuevo, encontramos que la implementación de una TH-IGIMF ayuda a reproducir de manera más natural las cantidades observadas y concluimos que las inestabilidades de disco juegan un rol preponderante en la formación de bulbos del tipo de la Vía Láctea. 



\section{Índice general}

Resumen

1. Introducción 1

1. Evolución química de galaxias y sus componentes . . . . . . . . . . 1

2. Marco Teórico 5

1. Paradigma $\Lambda \mathrm{CDM}$ de formación de estructura en el Universo $\ldots . . .5$

1.1. Historia del modelo $\Lambda \mathrm{CDM} \ldots \ldots \ldots \ldots$

1.2. Formación de halos de MO . . . . . . . . . . . . . . 15

1.3. Simulaciones cosmológicas de $N$-cuerpos . . . . . . . 26

3. Modelo semianalítico SAG 31

1. Tipos de galaxias y fusiones . . . . . . . . . . . . . 32

2. Enfriamiento radiativo del gas caliente . . . . . . . . . . . . 34

3. Formación Estelar . . . . . . . . . . . . . . . . . . . . . 35

4. Formación de discos . . . . . . . . . . . . . . . . 36

5. Formación de esferoides: bulbos y galaxias elípticas . . . . . . . . . 36

6. Retroalimentación por supernovas . . . . . . . . . . . . . . 38

7. Retroalimentación por núcleos de galaxias activos . . . . . . . . . 38

8. Incorporaciones al modelo . . . . . . . . . . . . . . . . . . . . 39

8.1. Modelado químico, nuevos yields . . . . . . . . . 40

8.2. Una nueva teoría de IMF Variable . . . . . . . . . . . . . . . 41

9. Brotes extendidos en SAG . . . . . . . . . . . . . . . . . 45

10. Propiedades Espectro-Fotométricas con la TH-IGIMF . . . . . . . . . 46

11. Calibración del modelo . . . . . . . . . . . . . . . . . . . . . 48 
4. Downsizing quemo-arqueológico 49

1. Introducción . . . . . . . . . . . . . . . . . . . . . . 49

2. Construcción de la relación $[\alpha / \mathrm{Fe}]$-masa estelar $\ldots \ldots \ldots \ldots \ldots$

3. Calibraciones ...................... 54

3.1. Restricciones observacionales . . . . . . . . . . . . 54

3.2. Resultados de las calibraciones . . . . . . . . . . . . . . 56

4. Relación $[\alpha / \mathrm{Fe}]$-masa: Impacto de una TH-IGIMF $\ldots \ldots \ldots \ldots$

4.1. Impacto de una TH-IGIMF . . . . . . . . . . . . . . . . 63

5. Brotes extendidos y escala temporal de explosión de SNs _ . . . . . . 69

5.1. Brotes extendidos: diferentes suposiciones sobre la duración inicial del brote . . . . . . . . . . . . . . . . . . 69

5.2. Escala temporal de explosión de SNs . . . . . . . . . 75

5.3. Otras pruebas: cocientes $\mathrm{M} / \mathrm{L}$ y funciones luminosidad . . . . 78

6. $[\alpha / \mathrm{Fe}]-m a s a$ estelar: influencia de las escalas de tiempo de formación . 79

7. Discusión . . . . . . . . . . . . . . . . . . . 90

5. PEs del bulbo de galaxias tipo VL 95

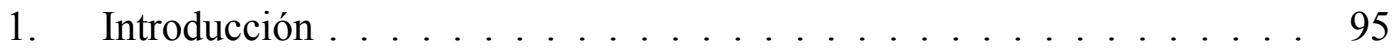

2. Selección de galaxias tipo $\mathrm{VL} \ldots \ldots \ldots$

3. Poblaciones estelares en un modelo semianalítico . . . . . . . . . . 104

4. Resultados . . . . . . . . . . . . . . . . . . 105

4.1. Distribución de metalicidades de PEs del bulbo . . . . . . . . 105

4.2. Abundancias de elementos- $\alpha$ en las PEs del bulbo . . . . . . . 119

4.3. Historias de formación y evolución de la metalicidad . . . . . . 124

4.4. Discusión . . . . . . . . . . . . . . . . . . 128

$\begin{array}{lr}\text { 6. Conclusiones } & 131\end{array}$ 
Dedicada a mi mamá y hermanos, a Olgui y a los que ya no están 



\title{
Capítulo 1
}

\section{Introducción}

\section{Evolución química de galaxias y sus componentes}

\author{
"if you wish to make an apple pie [galaxy] from scratch, \\ you must first invent the universe"
}

-Carl Sagan, Cosmos.

Las galaxias son los bloques constitutivos del Universo. Conocer en detalle los procesos que suscitan su formación y su posterior evolución es uno de los problemas fundamentales de la astronomía moderna. Una de las herramientas utilizadas para contribuir al armado de este complejo rompecabezas es el estudio de las propiedades químicas de las galaxias y sus componentes.

Los diferentes metales ${ }^{1}$ que componen la materia bariónica del Universo se forman en las estrellas por nucleosíntesis. Estos elementos químicos son eyectados al medio interestelar por vientos y explosiones de supernova ( $\mathrm{SN}$ ), se diluyen, y posteriormente son incorporados a las sucesivas generaciones de estrellas y planetas que se forman a partir del gas contaminado. Las abundancias iniciales de elementos químicos de las estrellas, definidas por las abundancias de este gas del cual se forman, permanecen como fósiles durante la mayor parte de su tiempo de vida y pueden ser medidas a través de los índices espectrales, calculados usando las mediciones de líneas en los espectros obtenidos

\footnotetext{
${ }^{1}$ Un metal es todo elemento químico con número atómico mayor que 2. Quedan excluidos el $\mathrm{H}$, el He y el Li.
} 
a partir de la luz que emiten las estrellas. Cuando se trata de sistemas estelares, como las galaxias, podemos observar su luz integrada e inferir las abundancias químicas del conjunto de estrellas que las forman.

Las propiedades químicas de las galaxias tienen una importancia fundamental para la teoría de formación y evolución de las galaxias. Por un lado, son una herramienta poderosa a la hora de reconstruir su historia de formación. Las galaxias son sistemas estelares de una gran complejidad, compuestas por múltiples poblaciones estelares, cada una formada en instantes de tiempo y en entornos diferentes y con su propia huella química. A partir de las metalicidades integradas de las galaxias, gradientes de metalicidad en función de la distancia galactocéntrica o estudios químicos de sus componentes, es posible reconstruir detalles sobre su historia de formación.

Por otro lado, muchos de los procesos que intervienen en la formación de las galaxias, como el enfriamiento del gas y la consecuente formación estelar, son dependientes de la metalicidad. Incluso, las propiedades espectrofotométricas de las galaxias no sólo dependen de la edad de las estrellas sino también del contenido de metales de las mismas.

Las propiedas químicas observadas en las galaxias establecen restricciones que guían el modelado de los procesos físicos que intervienen en su enriquecimiento químico y, por lo tanto, en su formación y evolución. El objetivo de un modelo de formación y evolución de galaxias es reproducir la mayor cantidad de propiedades observadas, permitiendo evaluar el impacto de distintos aspectos del modelo en los resultados obtenidos. La implementación en tal modelo de un esquema que describa el enriquecimiento químico de las galaxias permite analizar el escenario de formación y evolución de las mismas desde un punto de vista específico, contribuyendo a la comprensión general de este complejo fenómeno.

Los ingredientes básicos de cualquier modelo de enriquecimiento químico de galaxias son:

\section{- Las condiciones iniciales:}

El gas a partir del cual se forman las galaxias tiene una composición química inicial. La cosmología moderna tiene entre sus temas de estudio la nucleosínte- 
sis primordial y da una respuesta a este interrogante. La composición química comúnmente aceptada es de aproximadamente $74 \%$ de hidrógeno y $26 \%$ de helio más porcentajes mínimos de deuterio, litio y algunos isótopos.

\section{- Formación estelar:}

Las recetas de formación estelar establecen la cantidad de estrellas formadas en diferentes momentos y contextos y determinan la historia de contaminación química de las estrellas. Los modelos analíticos de evolución química recurren a diferentes recetas de formación estelar en el tiempo. En los modelos semi-analíticos, como el que utilizamos en el presente trabajo de tesis, la formación estelar ocurre en un contexto cosmológico por diferentes procesos. Sigue una evolución estocástica y no suave.

\section{- Yields estelares:}

La teoría de la evolución estelar es una de las ramas de la astronomía de mayor desarrollo. Existen modelos de evolución de estrellas en todo el intervalo de masas, desde estrellas de masa menor que la del Sol, hasta estrellas supermasivas que terminan su vida como SNs o de estrellas de población III. Aunque el campo sigue siendo de una actividad intensa, hoy en día existen en la literatura muchos conjuntos de datos sobre la cantidad de metales que eliminan al medio interestelar los distintos tipos de estrellas durante su vida y luego de finalizarla, cantidad conocida como yields estelares.

\section{- Función inicial de masa:}

Una vez establecido qué cantidad de metales eyecta cada tipo de estrella y qué cantidad de estrellas se forman por unidad de tiempo, es necesario conocer la función inicial de masa (IMF, por su sigla en inglés), la cual establece la distribución inicial de masas de las estrellas que surgen de un evento de formación estelar. De este modo, es posible estimar el enriquecimiento químico que sufre una galaxia a lo largo de su vida. La IMF en cúmulos estelares es bien conocida. Cuando se trata de sistemas estelares compuestos como las galaxias la discusión está abierta y se acumulan evidencias año a año que parecen indicar que esta distribución fundamental no es la misma de galaxia a galaxia.

En el presente trabajo de tesis utilizaremos el modelado químico contenido en un 
modelo semi-analítico de formación y evolución de galaxias en el marco del paradigma cosmológico actual, introducidos en los capítulos 2 y 3 , para investigar los patrones de abundancias de las galaxias elípticas, y de los bulbos de las galaxias semejantes a nuestra Vía Láctea. Lo primero nos ayudará a restringir los mecanismos de formación de esferoides y analizar los efectos de distintas alternativas para la representación de la función inicial de masa integrada para las galaxias. En el camino contestaremos algunos interrogantes sobre las abundancias de elementos $\alpha$ en este tipo de galaxias. Estos elementos, llamados de esta manera porque sus núcleos contienen múltiplos de partículas $\alpha$ (núcleos completamente ionizados de helio), son producidos casi exclusivamente en SNs tipo II (SNs II) y son utilizados para determinar escalas de tiempo de formación, como será detallado en el capítulo 4. En el capítulo 5, nos concentraremos en los bulbos de galaxias tipo Vía Láctea, e intentaremos ahondar en los posibles escenarios de formación del bulbo de nuestra galaxia a través de sus propiedades químicas. Finalmente, se presentarán las conclusiones. 


\title{
Capítulo 2
}

\section{Marco Teórico: Formación de estructura a gran escala}

\author{
'Cold Dark Matter theory has now reached the point at \\ which it should be admitted as a Candidate Member to \\ the Academy of Established Theories, so that it can sit \\ alongside the established theories of Maxwell, Einstein, \\ and Heisenberg and be used as a standard tool in the \\ construction of new theories'
}

-James Binney, 2003.

En este capítulo se realiza una descripción del marco teórico utilizado para la concreción del presente trabajo. Presento un breve recorrido histórico por los avances que dieron lugar al paradigma actual de formación de estructura a gran escala y se describe la simulación de $N$-cuerpos utilizada a lo largo del trabajo.

\section{Paradigma $\Lambda \mathrm{CDM}$ de formación de estructura en el Universo}

La tesis se enmarca dentro del paradigma actual de formación de estructuras a gran escala del Universo, conocido como modelo cosmológico de concordancia de materia 
oscura fría con constante cosmológica, $\left(\Lambda \mathrm{CDM}\right.$, por sus siglas en inglés $\left.{ }^{1}\right)$. El modelo $\Lambda \mathrm{CDM}$ se apoya sobre mediciones muy precisas del fondo cósmico de radiación (Tegmark et al., 2004; Dunkley et al., 2009; Planck Collaboration et al., 2014) que permiten calcular los parámetros importantes presentes en la teoría. A partir de los últimos parámetros cosmológicos presentes en la literatura, se desprende que el 68,3\% de la densidad de materia-energía del Universo se encuentra dominada por la energía oscura y un $31,7 \%$ por materia. Este porcentaje se desdobla en un $26,8 \%$ correspondiente a la materia oscura (MO), y un 4,9\% restante correspondente a material bariónico.

En este modelo, se supone que durante el período inflacionario se producen fluctuaciones cuánticas que generan pequeñas sobredensidades en la materia, que se encontraba uniformemente distribuida en el Universo primigenio. Las fluctuaciones en el campo de densidad crecen luego por inestabilidad gravitacional agregando materia circundante. Eventualmente, cuando sus amplitudes son lo suficientemente grandes, se desprenden del proceso de expansión del Universo, colapsan y forman estructuras en cuasi equilibrio virial. De este modo, se forman sistemas densos, a los que se denomina halos de materia oscura (HMO). Estos halos constituyen el sitio de formación de galaxias individuales. A su vez, los HMO sufren un proceso de agregación jerárquica. En el modelo $\Lambda \mathrm{CDM}$, las estructuras más pequeñas se fusionan para dar lugar a estructuras más grandes. De esta manera, se forman en el tiempo halos de tamaño cada vez mayor, donde habitan los grupos y cúmulos de galaxias. Sin embargo, la formación y evolución de estos HMO resultan en un proceso complejo y altamente no lineal, donde coexisten distintas escalas de amplitud de las sobredensidades a lo largo de la evolución del Universo. Por ejemplo, los cúmulos de galaxias son los objetos que más recientemente han alcanzado la relajación gravitacional, pero sin embargo son los que más temprano han empezado su formación como objeto de escala subgaláctica; además los últimos eventos de fusión sufridos por un cúmulo de galaxias fueron probablemente acreciones de halos pequeños. El gas presente en la distribución de materia original colapsa siguiendo la dinámica dada por las estructuras de materia oscura y experimenta calentamiento por choque al caer en los pozos de potencial determinados por los HMO. Posteriormente, el gas sufre diferentes procesos de enfriamiento radiativo y colapsa conservando su momento angular, dando lugar a la formación de discos fríos con la densidad suficiente para producir frag-

\footnotetext{
${ }^{1}$ A lo largo del trabajo de tesis se tomá la convención de utilizar siglas en inglés para los casos en que está muy extendido su uso en la bibliografía.
} 
mentaciones que conducen a eventos de formación estelar. Las fusiones entre HMO, con sus respectivas galaxias, y diversos mecanismos físicos dan lugar al amplio "zoológico" de galaxias observadas. La riqueza y complejidad de estos fenómenos y las enormes escalas temporales y espaciales involucradas en la formación y evolución de las galaxias hacen que este campo de investigación se relacione con muchos otros campos de la astronomía y sea uno de las más activos de la astronomía actual. A continuación, se realizará un breve recorrido por las ideas que forjaron el paradigma actual de formación y evolución del Universo.

\subsection{Historia del modelo $\Lambda \mathrm{CDM}$}

En 1933, Fritz Zwicky escribió un famoso artículo en el cual postula por primera vez la existencia de una gran cantidad de materia no visible en el cúmulo de galaxias Coma (Zwicky, 1933). El trabajo de investigación está dedicado a la medición de las propiedades de una gran parte de las nubes extragalácticas, como se le llamaba a las galaxias en esa época, conocidas hasta el momento. Zwicky midió y analizó distancias y velocidades radiales. En una de las secciones aplicó el teorema del virial a las 8 galaxias con velocidades medidas pertenecientes al cúmulo de Coma y encontró que la densidad media del sistema, y por lo tanto su masa, debía ser 400 veces mayor para explicar la dispersión de velocidades observada, de alrededor de $1000 \mathrm{~km} \mathrm{~s}^{-1}$. Los valores actuales de la dispersión de velocidades de las galaxias en Coma son similares a los obtenidos por Zwicky (Colless \& Dunn, 1996). Sobre el final del cálculo, Zwicky concluye Falls sich dies bewahrheiten sollte, würde sich also das überraschende Resultat ergeben, dass dunkle Materie in sehr viel grösserer Dichte vorhanden ist als leuchtende Materie. ${ }^{2}$ El valor de 400 veces la densidad media resulta exagerado para los cocientes masa-luminosidad (M/L) medidos en la actualidad, $M / L \approx 50$. La razón de esta discrepancia radica en el valor de la constante de Hubble utilizado por Zwicky, $H_{0}=558 \mathrm{~km} \mathrm{~s}^{-1} \mathrm{Mpc}^{-1}$, que se correspondía con el valor calculado por el mismo Edwin Hubble utilizando las mediciones de velocidad de recesión de galaxias observadas y que nadie se atrevía a poner en duda, dada la reputación del astrónomo del momento, aún cuando estos resultados de densidad total del cúmulo parecieran extravagantes.

\footnotetext{
${ }^{2} \mathrm{Si}$ esto [la sobredensidad] es verificado, se daría el sorprendente resultado en el que la materia oscura está presente con mucha mayor densidad que la materia luminosa.
} 
En un artículo posterior publicado en The Astrophysical Journal, Zwicky se dedica de manera exclusiva a investigar el problema de las masas de las galaxias y los cúmulos de galaxias (Zwicky, 1937b). A pesar de la crisis económica reinante en Estados Unidos y en gran parte del mundo luego de la gran depresión, con parte del dinero de la familia de la primera mujer de Zwicky (Dorothy Vernon Gates) fue posible montar el telescopio Schmidt en el monte Palomar, con el cual se observaron las galaxias del cúmulo de Coma con una claridad sin precedentes. En este trabajo discute varios métodos para la determinación de masas de galaxias y menciona también, por primera vez, la posibilidad de utilizar lentes gravitacionales para este fin. Ese mismo año publicó una letter en el Physical Review donde describe con más detalle la utilización de lentes gravitacionales para la determinación de masas de galaxias (Zwicky, 1937a).

Sin embargo, recién en 1979 se descubrieron las primeras lentes gravitacionales que Einstein había predicho que serían muy difíciles de detectar directamente. Walsh et al. (1979) observaron el quasar $0957+561$ A, B, duplicado por la lente gravitacional provocada por un objeto masivo que se interponía con la visual, confirmando las predicciones realizadas por Zwicky en cuanto a la cierta probabilidad de observar este fenónemo cuando se interponía una galaxia masiva o un cúmulo de galaxias ante un objeto. Hoy en día, las lentes gravitacionales representan el mayor obstáculo de las teorías alternativas a la materia oscura para explicar el exceso aparente de materia invisible. Un ejemplo de estas teorías es la dinámica de newton modificada (MOND, por sus siglas en inglés), que logra explicar la dinámica de galaxias en cúmulos y las velocidades de rotación de los discos galácticos, pero no puede resolver la existencia de lentes gravitacionales, ni la órbita de Mercurio. Una clásica evidencia mencionada comúnmente sobre la existencia de materia oscura en galaxias está relacionada con la velocidad de rotación de objetos en regiones alejadas del centro galáctico. Fue Babcock (1939) el primero en encontrar una curva de rotación galáctica que no decrecía con el radio, como es de esperar en un comportamiento newtoniano clásico. El autor estudió la velocidad de rotación de la galaxia de Andrómeda utilizando espectroscopía de nubes moleculares a diferentes distancias del centro galáctico, encontrando una velocidad circular casi constante hasta radios muy grandes. El cálculo del cociente $\mathrm{M} / \mathrm{L}$ que realizó daba como resultado un valor de 50, muy por encima de los valores medidos en la vecindad solar. Si bien la evidencia de la existencia de materia oscura tiene muchas décadas, el concepto tal cual hoy lo conocemos es bastante más moderno. Zwicky mencionaba la posibilidad de una 
alta absorción en los cúmulos que no permitía observar la totalidad de la materia involucrada. Babcock pensó que la inusual velocidad en el disco exterior de Andrómeda era consecuencia de la existencia de materia cuya luz era absorbida en el exterior del disco de la galaxia. De la misma manera, Kahn \& Woltjer (1959) descubrieron que Andrómeda estaba orbitando nuestra galaxia y, realizando estimaciones cinemáticas, calcularon una masa para el sistema muy superior a la observada, concluyendo que la mayor parte de la masa del sistema estaría en alguna forma no visible, como gas caliente. En definitiva, se aceptaba la existencia de materia no visible alrededor de galaxias y cúmulos de galaxias, pero todavía se confiaba en que fuera simplemente materia que no brillaba lo suficiente para ser detectada. De hecho, tampoco se asociaban estas distintas observaciones a un mismo fenómeno (ni Babcock ni Khan \& Woltjer citaron a Zwicky o se citaron entre ellos). Iban a pasar muchos años hasta que se asociara la existencia de materia oscura a un nuevo tipo de materia, que no interacciona con la materia bariónica electromagnéticamente.

El acuñamiento del término materia oscura fría se lo adjudica normalmente a Bond \& Szalay (1983). Los autores de este trabajo estudian la materia oscura bajo la suposición de que es algún tipo de materia no-colisional, sujeta a las restricciones que imponía la nucleosíntesis de elementos ligeros a la existencia de materia oscura en forma de gas o planetas masivos, según ellos mismos habían calculado (Carr et al., 1982), y las observaciones de las curvas de rotación que indicaban que el cociente $\mathrm{M} / \mathrm{L}$ crecía con el radio. A diferencia de la materia no-colisional, el gas bariónico choca, se calienta, emite radiación y se enfría dinámicamente. Luego, se concentra hacia el centro del pozo de potencial. Esto genera que la concentración de materia oscura a mayor distancia del centro de las galaxias sea relativamente mayor que la de material bariónico. En el trabajo se discute la naturaleza de las partículas de materia oscura y se proponen como candidatos un tipo de neutrino masivo, para los cuales los cálculos estimaban que podrían acumularse en escalas grandes, $\mathrm{y}$ una partícula que llamaron $\mathrm{X}$ que podía aglomerarse a menores escalas espaciales dada su menor dispersión de velocidades. Los autores señalan: We may take all particles which decouple when they are norelativistic as being cold, and ignore all velocity dispersion effects. ${ }^{3}$

Ésta es la primera alusión a materia oscura fría en el sentido en que la conocemos

\footnotetext{
${ }^{3}$ Podemos considerar a todas las partículas que se desacoplan cuando son no-relativistas como frías, e ignorar todos los efectos de dispersión de velocidades.
} 
hoy, materia no-colisional de origen no bariónico con velocidades relativamente bajas y partículas relativamente masivas. Así, este trabajo es uno de los primeros en ordenar los conocimientos que se habían adquirido en décadas pasadas y clasificar a las partículas candidatas a formar la materia oscura en categorías de acuerdo al efecto que ocasionan en el espectro de fluctuaciones.

Los diferentes candidatos dan lugar así a 3 tipos de materia oscura (fría, tibia o caliente) de acuerdo a la velocidad a la que se movían por movimientos aleatorios en el Universo temprano, hasta cuando se desacoplaron de la radiación. La distancia recorrida en este intervalo de tiempo se conoce como longitud de corriente libre (free streaming) y su importancia radica en que las fluctuaciones primordiales cuya escala es menor que esta longitud son borradas y la materia no se aglomera en torno a ellas. De esta manera, mientras más "fría" es la materia oscura, más pequeña es la longitud de free streaming y, por lo tanto, más pequeña es la escala de aglomeración de la materia.

Las implicancias cosmológicas de la naturaleza de las partículas de materia oscura son enormes ya que determina cómo evoluciona la aglomeración de materia oscura durante la expansión del Universo desde pequeñas escalas hasta las grandes escalas. Esta discusión no pasó desapercibida en el siglo XX, en el tiempo anterior al trabajo de Bond y durante algo más de una década se debatió sobre dos modelos de crecimiento de las estructuras a gran escala. Einasto (2010) resume, en el marco de una conferencia para celebrar el cumpleaños 95avo. de Yákov Borísovich Zeldovich, uno de los primeros pasos que se dieron en la discusión sobre la importancia de la materia oscura en la cosmología. En 1975, se realizó la primer conferencia dedicada íntegramente a la materia oscura en Tallin, Estonia. En esta primer conferencia histórica se discutió con mayor énfasis que nunca hasta ese momento sobre su naturaleza, y se evidenció que los modelos basados en materia gaseosa o estelar mostraban muchos problemas. La materia gaseosa, por ejemplo, era necesaria para explicar la proporción de galaxias con poco gas cerca del centro de los cúmulos frente a la mayor proporción de galaxias ricas en gas en las afueras (Einasto et al., 1974), pero era varios órdenes de magnitud insuficiente para explicar la dispersión de velocidades de las galaxias. La materia oscura en las teorías cosmológicas ya ocupaba a esta altura un rol preponderante y se debatían dos modelos diferentes sobre la formación de la estructura a escala cosmológica. Por un lado, estaba el modelo de panqueques concebido y defendido por Zeldovich (Zel'dovich, 1970), 
en el cual una gran estructura colapsaba en una dimensión, formando un panqueque, y luego se fraccionaba dando lugar a porciones de materia oscura más chicas. Por otro lado, existía el modelo de crecimiento en sentido creciente (bottom up), defendido por Peebles, en el cual las estructuras de pequeño tamaño colapsaban primero, en las 3 dimensiones, para luego aglomerarse en estructuras más grandes (Peebles, 1971).

En 1977 se realizó la primera conferencia dedicada a investigaciones sobre la estructura a gran escala del Universo en donde se discutieron resultados de las actualizaciones del catálogo de galaxias de Shapley-Adams, que incluía galaxias locales de hasta magnitud 13.5 (Sandage \& Tammann, 1981; de Vaucouleurs et al., 1976). La extensión del relevamiento y su visualización en diagramas permitió vislumbrar por primera vez la morfología característica de la distribución de galaxias en el Universo con filamentos, nodos de aglomeración y vacíos considerables. En la Fig. 2.1 se puede ver la distribución de partículas en la simulación realizada por el grupo de Zeldovich en 1975 y la distribución de galaxias en un intervalo de declinaciones del catálogo extendido de Shapley-Adams (Joeveer \& Einasto, 1978).

Otro evento histórico que se dio a partir de la conferencia fue la presentación del reporte de investigacón de Parijskij (1978) en el que buscaba fluctuaciones en la densidad (y temperatura) en el Universo temprano utilizando el mayor radiotelescopio de la época, RATAN-600. El instrumento tenía como límite superior para la detección de perturbaciones en la temperatura un valor de $10^{-4}$ y no halló fluctuaciones en ese orden. Zeldovich había calculado que las amplitudes de las fluctuaciones debían ser de al menos $10^{-3}$ teniendo en cuenta material bariónico, caso contrario la inestabilidad gravitacional no era suficiente para contrarrestar la expansión del Universo.

Esto llevo a los científicos a preguntarse sobre la naturaleza no bariónica de la materia oscura. Ya fue mencionado la propuesta de una partícula X y la hipótesis de los neutrinos como constituyentes de la MO. Sin embargo, a partir de estos datos, el tema tomó especial relevancia. Diferentes autores propusieron que la materia oscura estaba formada por neutrinos, que para ese entonces se sabía tenían una cierta masa por los experimentos de Lyubimov. El crecimiento de perturbaciones en un medio dominado por neutrinos podía comenzar mucho antes que la época de la recombinación dando tiempo a las perturbaciones a crecer lo suficiente como para favorecer la formación de estructuras. Con motivo de esta nueva línea de investigación se realizó una nueva conferencia en 

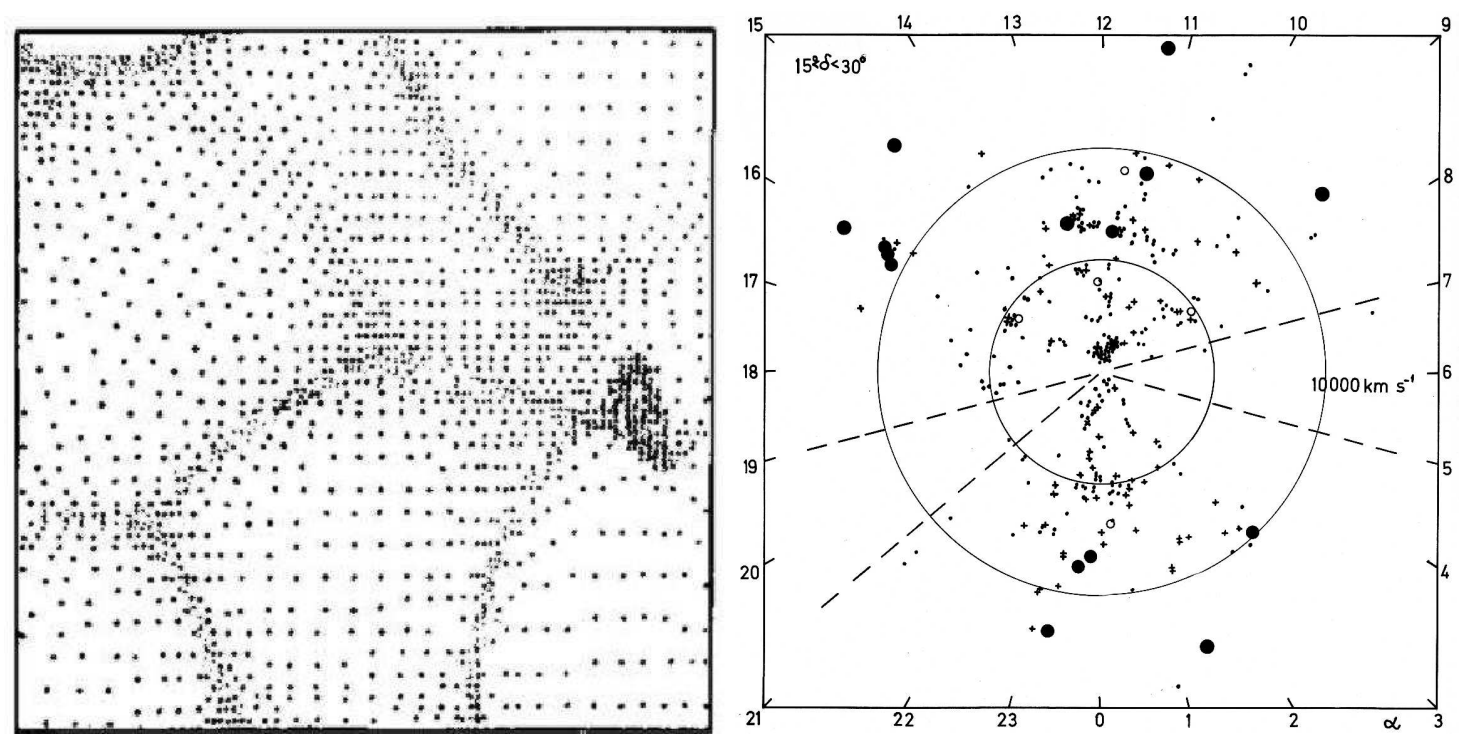

Figura 2.1: Izquierda: Distribución de partículas utilizando la aproximación de Zeldovich realizada por el grupo de investigación liderado por él mismo en 1975. Derecha: Diagrama con las posiciones de las galaxias del catálogo de galaxias extendido de Shapley-Adams. Los círculos llenos y abiertos indican cúmulos y grupos de galaxias, respectivamente, mientras que los puntos y cruces corresponden a galaxias y galaxias tipo Markarian.

Tallin, Estonia, en 1981, en donde Zeldovich dio un discurso en el que dijo: Observers work hard in sleepless nights to collect data; theorists interpret observations, are often in error, correct their errors and try again; and there are only very rare moments of clarification. Today it is one of such rare moments when we have a holy feeling of understanding the secrets of Nature. ${ }^{4}$ Las palabras retratan el momento de excitación al ser parte de la generación que se dio cuenta de que materia no bariónica es necesaria para comenzar la formación de estructura lo suficientemente temprano. Sin embargo, la materia oscura formada por neutrinos presentó sus problemas también. Las perturbaciones de pequeña escala son atenuadas (damped), lo que previene la formación de estructura fina como los filamentos y HMO del tamaño de galaxias. Además, las densidades muy

\footnotetext{
${ }^{4}$ Los observadores trabajan duro y sin dormir durante las noches para reunir datos; los teóricos interpretan las observaciones, se equivocan usualmente, corrigen sus errores y lo intentan nuevamente; y son muy poco frecuentes los momentos de clarificación. Hoy es uno de esos raros momentos cuando tenemos un sentimiento sagrado de entendimiento de los secretos de la naturaleza.
} 
altas de MO en galaxias enanas esferoidales en conjunción con el principio de exclusión de Pauli también imponían restricciones fuertes a los modelos de neutrinos (Tremaine \& Gunn, 1979). Estos problemas motivaron la formulación de nuevos escenarios de materia oscura en los cuales se proponían todo tipo de partículas como gravitinos, axiones, fotinos. Como fue mencionado antes en el capítulo, a estos nuevos modelos de materia oscura se los llamó de MO fría (CDM por sus siglas en inglés). Los modelos CDM fueron comparados con los de materia oscura caliente (HDM por sus siglas en inglés) en simulaciones realizadas por White et al. (1983, 1987). La comparación arrojaba que los modelos CDM, en ese momento con densidad de materia $\Omega_{m}=1^{5}$, reproducían mejor una serie de características de la distribución de materia a gran escala, con los filamentos, vacíos y cúmulos como se observaba.

Paralelamente al desarrollo de las ideas del paradigma CDM y el contenido de materia del Universo, se desarrollaba el estudio de la geometría del Universo como un todo. Albert Einstein había planteado la existencia de una constante en su conjunto de ecuaciónes tensoriales que relaciona la geometría con el contenido de materia del Universo por una cuestión de elegancia matemática. Más tarde se iba a arrepentir de su inclusión y considerar el hecho como el mayor error de su carrera. Durante buena parte del principio del siglo XX prevaleció la idea de un tipo de Universo llamado de Einstein-de Sitter, debido a que de Sitter fue el primero en resolver las ecuaciones de Einstein para un Universo de este tipo. Por la época, hasta los años 20, estaba en discusión, además, si las nebulosas espirales, ahora correctamente llamadas galaxias espirales, estaban contenidas en nuestra galaxia o eran sistemas estelares extragalácticos. El descubrimiento de estrellas cefeidas en M31 y M33 (Hubble, 1925) y de una relación lineal de la velocidad de recesión de las galaxias en el Universo local con su distancia (Hubble, 1929; Hubble \& Humason, 1931) llevaron a la comunidad científica a reconocer que el Universo era más vasto de lo que se creía y que estaba en expansión. Esta concepción del Universo implicaba que el espacio-tiempo se expandía desde un momento en particular en el que toda la materia se viera concentrada en un mismo lugar, una singularidad en las ecuaciones de Einstein. Esta particularidad llevó a Fred Hoyle, uno de los mayores detractores de este modelo, a ridiculizarlo nombrando al primer instante de expansión como big

\footnotetext{
${ }^{5}$ La densidad de materia es normalizada por la densidad crítica del Universo para la cual la geometría es plana, $\Omega_{m}=\rho_{m} / \rho_{c}$. La densidad crítica total del Universo, es la suma de la densidad de materia $\Omega_{m}$, la densidad de energía oscura $\Omega_{\Lambda}$ y la densidad de radiación $\Omega_{R}$.
} 
bang, una gran explosión, denominación que tiene hasta la actualidad. La discusión sobre la naturaleza de la expansión del Universo siguió presente hasta los trabajos de dos grupos de investigación independientes sobre supernovas tipo Ia (SNIa) que valieron un premio Nobel de física a Saul Perlmutter, Brian P. Schmidt y Adam G. Riess en 2011. En esta serie de trabajos (e.g. Perlmutter et al., 1999; Schmidt et al., 1998), se observó que la luminosidad de las SNIa decrecía para aquellas más lejanas. La luminosidad de las SNIa, que es la explosión de una enana blanca que acreta materia de su compañera, es intrínsecamente la misma. Lo observado podía ser explicado si el Universo se estaba expandiendo aceleradamente, descartando que algún proceso físico pudiera reducir la luminosidad de las SN Ia de alto redshift (corrimiento al rojo, utilizado comúnmente como medida de distancia, dada la relación que existe entre el corrimiento al extremo rojo de la radiación, y como medida del instante en la vida del Universo de algún evento particular, ver e.g. Hogg, 1999). Esta posibilidad ya había sido contemplada en los modelos de formación de estructura. Argumentos a favor de un modelo con constante cosmológica ya habían sido sugeridos por Gunn \& Tinsley (1975) y los modelos con esta constante se mostraron superiores, ya que el crecimiento de las fluctuaciones no debía cortarse de manera artificial como en los modelos con $\Omega_{m}=1$. Otros modelos propuestos como los de materia tibia, compuestos por parte de materia oscura fría y otro porcentaje por partículas más energéticas, también fueron descartados en la década del 90 porque formaban estructuras demasiado tarde, ya que con observaciones a alto redshift se constata la existencia de cúmulos y grupos de galaxias en etapas demasiado tempranas del Universo para los parámetros de estos modelos. Los modelos basados en una cosmología $\Lambda \mathrm{CDM}$ funcionan bien en muchos aspectos y combinan las características esenciales de los modelos de formación de estructura de panqueques y del modelo de agregación jerárquica.

Hoy en día se han realizado grandes relevamientos de galaxias para mapear la distribución de materia en el Universo, como es el caso del Sloan Digital Sky Survey (SDSS, York et al., 2000) que involucra casi un cuarto del total del cielo y relevó la posición y datos fotométricos de galaxias en 5 bandas hasta una magnitud $\mathrm{r}=23$, y los espectros y redshifts de galaxias hasta magnitud $\mathrm{r}=17.77$. Por otro lado se ha logrado simular la formación de estructura a gran escala en grandes volúmenes que utilizan algunas de las computadoras con mayor poder de cálculo que (e.g. la simulación Bolshoi y Millenium 

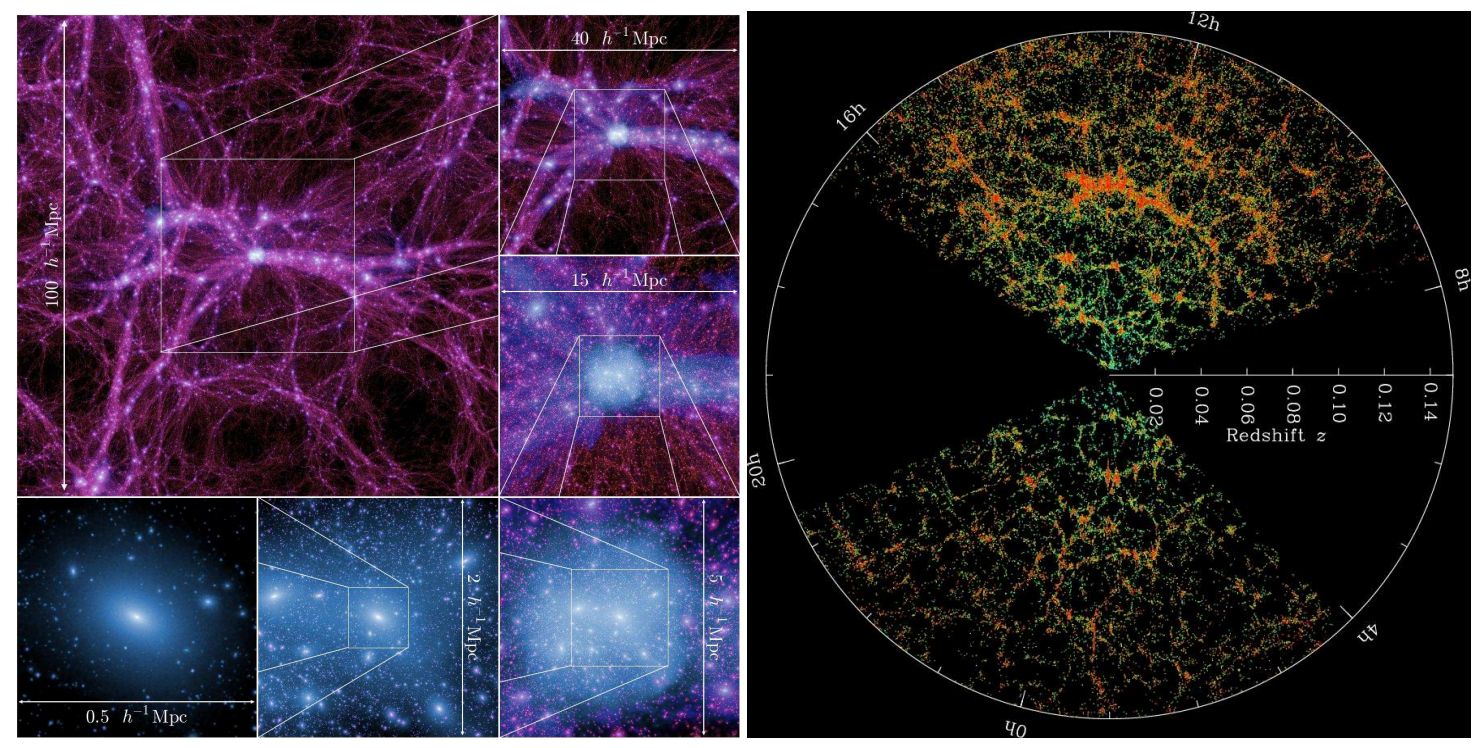

Figura 2.2: Izquierda: Distribución de partículas de simulación de $N$-cuerpos realizada por el consorcio Millenium II. Los diferentes cuadros muestran distintas escalas de la caja simulada. Derecha: Diagrama que muestra la posición y redshift de las galaxias observadas en el relevamiento SDSS. Imagen tomada del sitio oficial del relevamiento, basada en la última liberación pública de datos. Cada punto es una galaxia. La escala de colores indica la densidad de puntos observada.

II, Klypin et al., 2011; Boylan-Kolchin et al., 2009). En la Fig. 2.2 se pueden ver los resultados del SDSS y de las últimas simulaciones disponibles (Millenium II). El nivel de detalle de los resultados mostrados en la Fig. 2.2 ha permitido un avance considerable en el desarrollo de la teoría de formación de galaxias en el marco del paradigma $\Lambda \mathrm{CDM}$.

\subsection{Formación de halos de MO}

En esta sección se hace mención a los conceptos básicos relacionados con la formación de halos de materia oscura. Algunas de las secciones contienen resúmenes de partes del capítulo 7 del libro "Galaxy Formation and Evolution" de Mo, Van den Bosh $\&$ White (2010) y representan una selección de los aspectos más relevantes en la formación de HMO según el criterio del autor de la tesis. 


\section{Crecimiento lineal de las perturbaciones cosmológicas}

El principio cosmológico reza que el Universo es homogéneo e isotrópico. Puede corroborarse que este enunciado es correcto con gran precisión a través del estudio de relevamientos de galaxias en escalas del orden o mayores a $70 \mathrm{~h}^{-1}$ Mpc (Sarkar et al., 2009), donde h es el factor de Hubble, definido como $\mathrm{h}=\frac{H_{0}}{100 \mathrm{kms}^{-1} \mathrm{Mpc}^{-1}}$

Sin embargo, a escalas pequeñas la materia se aglomera en galaxias, las cuales se aglomeran en grupos o cúmulos mientras que otras regiones del Universo permanecen "vacías", con las galaxias apartándose de ellas. Estas estructuras crecen por la atracción gravitacional a partir de pequeñas fluctuaciones iniciales. Se cree que estas fluctuaciones tienen un origen cuántico y que luego son amplificadas por la inflación hasta el orden de $10^{-5}$, medidas en la temperatura del fondo cósmico de microondas. Como las perturbaciones inicialmente son pequeñas, al menos durante un período de tiempo, el crecimiento en un Universo en expansión se puede tratar como lineal y utilizando un formalismo clásico, no relativista. Cuando las perturbaciones crecen más allá de un límite en densidad $\left(\frac{\delta \rho}{\rho} \approx 1\right)$, su crecimiento se vuelve no lineal y es necesario recurrir a integraciones numéricas para estudiar la dinámica de la materia oscura.

El tratamiento usual es el tratamiento perturbativo de un fluido autogravitante con un término de expansión del Universo. Para ello se resuelven las ecuaciones de continuidad, de Euler, de la conservación de la energía y de Poisson. En formulación Euleriana:

$$
\begin{gathered}
\frac{\partial \rho}{\partial t}+\nabla(\rho \mathbf{v})=0, \\
\frac{\partial(\mathbf{v})}{\partial t}+(\mathbf{v} \cdot \nabla) \mathbf{v}=-\frac{1}{\rho} \nabla p-\nabla \Phi, \\
\rho \frac{\partial}{\partial t} u+\rho(\mathbf{v} \cdot \nabla) u=-p \nabla \mathbf{v}, \\
\nabla^{2} \Phi=4 \pi G \rho,
\end{gathered}
$$

donde $u$ es la energía por unidad de masa, $u=p /(\gamma-1) \rho, v$ es la velocidad del fluido, p su presión, $\rho$ la densidad y $\Phi$ el potencial. El fluido en este caso debe considerar la expansión del Universo, para lo cual se pueden escribir la posición y la velocidad como 
perturbaciones sobre una expansión homogénea. Para ello se introduce la posición comóvil $\mathbf{x}$ y la velocidad peculiar $\mathbf{v}_{p}$ :

$$
\begin{gathered}
\mathbf{r}=a(t) \mathbf{x}, \\
\mathbf{v}=\dot{r}=\dot{a} \mathbf{x}+a \dot{\mathbf{x}} \equiv \dot{a} \mathbf{x}+\mathbf{v}_{p},
\end{gathered}
$$

donde $a(t)$ es el factor de escala del modelo cosmológico en expansión. La velocidad $\mathbf{v}$ es la suma de la velocidad de Hubble $\dot{a} \mathbf{x}=H \mathbf{r}$ y una velocidad peculiar. Luego se reemplaza $\mathbf{r}$ por las coordenadas co-móviles $\mathbf{x}$ en las ecuaciones y se escribe la densidad como:

$$
\rho(\mathbf{x}, t)=\rho_{b}(t)(1+\delta(\mathbf{x}, t))
$$

donde $\rho_{b}(t)$ describe la evolución temporal del Universo sin perturbar y $\delta$ la desviación de la solución homogénea. Haciendo álgebra con las mismas y usando que el factor de escala satisface las ecuaciones de Friedmann, llegamos a un conjunto de ecuaciones para un fluido autogravitante en un Universo en expansión que luego de linealizarlas tienen la forma:

$$
\begin{gathered}
\dot{\boldsymbol{\delta}}+\nabla \dot{\mathbf{x}}=0, \\
\ddot{\mathbf{x}}+2 H \dot{\mathbf{x}}=-\frac{1}{a^{2}} \nabla \Psi-\frac{v_{2}^{2}}{a^{2}} \nabla \delta, \\
\nabla^{2} \Psi=4 \pi G \rho_{b} \delta a^{2} .
\end{gathered}
$$

Tomando la derivada temporal de la primera, y la divergencia de la segunda, se simplifica a

$$
\ddot{\delta}+2 H \dot{\delta}-\frac{v_{s}^{2}}{a^{2}} \nabla^{2} \delta=4 \pi G \rho_{b} \delta .
$$

Para un fluido auto-gravitante sin expansión las soluciones son de tipo exponencial, ya que $H=0$. Con la dependencia explícita de $H$ con el tiempo la situación es distinta. Un ejemplo típico es el Universo de Einstein-De Sitter, en el cual $a \propto t^{2 / 3}$ de manera que:

$$
a=a_{0}\left(\frac{t}{t_{0}}\right)^{2 / 3}
$$




$$
\begin{gathered}
H=\frac{2}{3 t}, \\
H=\frac{8 \pi G}{3} \rho_{b}=\frac{4}{9 t^{2}},
\end{gathered}
$$

en donde también se usó la segunda ecuación de Friedmann para $k=\Lambda=0$, donde k es la constante de curvatura y $\Lambda$ la constante cosmológica. Reemplazando $H$ en la ecuación 2.11 se encuentran dos soluciones: $\delta \propto t^{2 / 3}$ y $\delta \propto t^{-1}$, y por lo tanto la solución general resulta:

$$
\delta=A t^{2 / 3}+B t^{-1}=A a+B a^{-3 / 2}
$$

El primer término crece con el tiempo, mientras que el segundo decrece. La solución se comporta como una ley de potencias. La importancia de esto radica en que las perturbaciones inestables crecen "sólo" como una ley de potencias, mucho más lento que en el caso de una perturbación en un fluido sin expansión. En el caso general, el modo creciente se debe obtener numéricamente. Se demuestra que, teniendo una solución, la otra se puede obtener a través de una ecuación diferencial de primer orden:

$$
\delta_{2} \dot{\delta}_{1}-\delta_{1} \dot{\delta}_{2} \propto a^{-2}
$$

donde $\delta_{1}$ y $\delta_{2}$ son dos soluciones a la Ec. 2.11. Entonces, teniendo el modo creciente fácilmente se obtiene el decreciente. Para encontrar la solución general del modo creciente hay que recurrir al cálculo numérico, pero una buena aproximación fue obtenida por Carroll et al. (1992):

$$
\delta_{+} \propto D(z) \propto g(z) /(1+z)
$$

donde

$$
g(z)=\frac{5}{2} \Omega_{m}(z)\left\{\Omega_{m}^{4 / 7}(z)-\Omega_{\Lambda}(z)+\left[1+\Omega_{m}(z) / 2\right]\left[1+\Omega_{\Lambda}(z) / 70\right]\right\}^{-1}
$$

$\mathrm{D}(\mathrm{z})$ es conocida como la tasa de crecimiento lineal. Las perturbaciones tienen que colapsar en contra del movimiento de expansión del Universo. Este crecimiento lineal es válido sólo para $\delta<<1$. Cuando las perturbaciones alcanzan una densidad crítica 
en el instante de turn around (dar la vuelta en inglés, se refiere al instante en que un conjunto de partículas de MO logra una masa y densidad suficiente para contrarrestar la expansión del Universo), colapsan para formar HMO. Las perturbaciones lineales crecen más rápido en un universo de Einstein-De Sitter que en un universo con $\Omega_{m, 0}<1$. La razón física de esto es que en un universo abierto, o con constante cosmológica distinta de 0 , la tasa de expansión es más grande que un universo EdS y el crecimiento de las perturbaciones se ve reducido por el "arrastre" de la expansión.

El anterior desarrollo (incompleto) es parte de la teoría Newtoniana de pequeñas perturbaciones. Esta teoría es válida siempre y cuando (i) la escala de longitudes de las perturbaciones sean mucho más chicas que el tamaño del horizonte al momento en cuestión (así la causalidad puede ser considerada instantánea) y (ii) el contenido de materia del Universo se pueda tratar como un fluido no relativista. Si las perturbaciones fueran creadas a un tiempo temprano $t_{i}$, entonces todas las perturbaciones con tamaños $\lambda>c t_{i}$ habrían fallado en cumplir con la primer condición. La segunda condición no es cumplida para perturbaciones en partículas relativistas como los fotones y los neutrinos sin masa. Un desarrollo completo de la teoría relativista de perturbaciones se encuentra en el libro de Mo et al. (2010).

\section{Colapso gravitatorio del campo de densidad cósmico y aglomeración jerárquica}

Las perturbaciones del campo de densidad cósmico evolucionan y eventualmente sucede un colapso gravitacional de las fluctuaciones. Para saber qué tipo de estructuras pueden esperarse de esos colapsos, se pueden realizar simulaciones de $N$-cuerpos, pero con algunas consideraciones analíticas también podemos darnos una idea. Las perturbaciones de densidad crecen como $\delta(\mathbf{x}, t) \propto D(t)$ y entonces la varianza $\sigma^{2}(r ; t) \propto D^{2}(t)$, donde $\sigma^{2}(r ; t)$ es la varianza de la distribución de galaxias muestreadas con esferas de radio $\mathrm{R}$ aleatoriamente ubicadas, $\mathrm{y}$ se relaciona con el espectro de potencias. El espectro de potencias del campo de densidad inicial suele suponerse como una ley de potencias $P(k) \propto k^{n}$ y luego $\sigma^{2}(r) \propto r^{-(n+3)}$, de manera que podemos escribir:

$$
\sigma^{2}(r ; t)=\left[\frac{r}{r *(t)}\right]^{-(n+3)}=\left[\frac{M}{M *(t)}\right]^{-(n+3) / 3}
$$

donde 


$$
M^{*}(t) \propto[D(t)]^{6 /(n+3)}, r^{*}(t) \propto[D(t)]^{2 /(n+3)},
$$

son los valores cuando $\sigma=1$ a tiempo $t$. La sobredensidad crítica de colapso gravitatorio es $\approx 1,68$ de acuerdo al modelo de colapso esférico. Es de esperarse que estructuras con masas $\approx M$ se terminen de formar cuando el valor lineal de $\sigma(M ; t)$ alcance 1.68. Por eso, la dependencia temporal de $M^{*}$ se puede usar para entender cómo las estructuras no lineales se desarrollan con el tiempo para un dado espectro de potencias. $D(t)$ crece con el tiempo, entonces la masa de escala de no-linearidad crece con $t$ para $n>-3$ y la formación de estructura se produce de una manera "bottom-up", es decir, las estructuras pequeñas se forman antes que las grandes. Los modelos CDM tienen índices espectrales efectivos mayores a -3 para todas las escalas de longitud. En cambio, en los modelos HDM, en los cuales el índice espectral efectivo es $n<-3$ en escalas menores a la longitud de free streaming, las primeras estructuras en colapsar son panqueques con tamaños comparables a la longitud de free-streaming. De esta manera, las estructuras pequeñas evolucionarían a partir de las mayores, dando lugar a un escenario "top down"de formación de estructura.

Basados en estas consideraciones simples podemos dividir la evolución del campo de densidades cosmológico en 3 regímenes distintos. El primero, en escalas de masa $M>>M^{*}$, las fluctuaciones de densidad todavía están en el régimen lineal y su crecimiento está dominado por la teoría de las perturbaciones lineales. El segundo, en escalas de masa $M<<M^{*}$, las fluctuaciones han colapsado para formar objetos virializados cuyos perfiles de densidad están determinados por sus condiciones iniciales y por varios procesos de relajación. Finalmente, en las escalas intermedias $\left(M \approx M^{*}\right)$ las fluctuaciones están en un régimen cuasi lineal y la estructura estaría dominada por panqueques y filamentos. De esta manera se ve cómo a un dado tiempo, el campo de densidad cósmico es una complicada red formada por halos virializados, redes de filamentos y panqueques y grandes vacíos. Todas estas características se observan en las simulaciones autoconsistentes de materia oscura, como es el caso de la simulación Millenium II (Fig. 2.3).

\section{Picos de densidad}

En las secciones anteriores vimos que la evolución de las sobredensidades en el campo de densidad inicial tiene una etapa de evolución lineal en la cual el campo de 

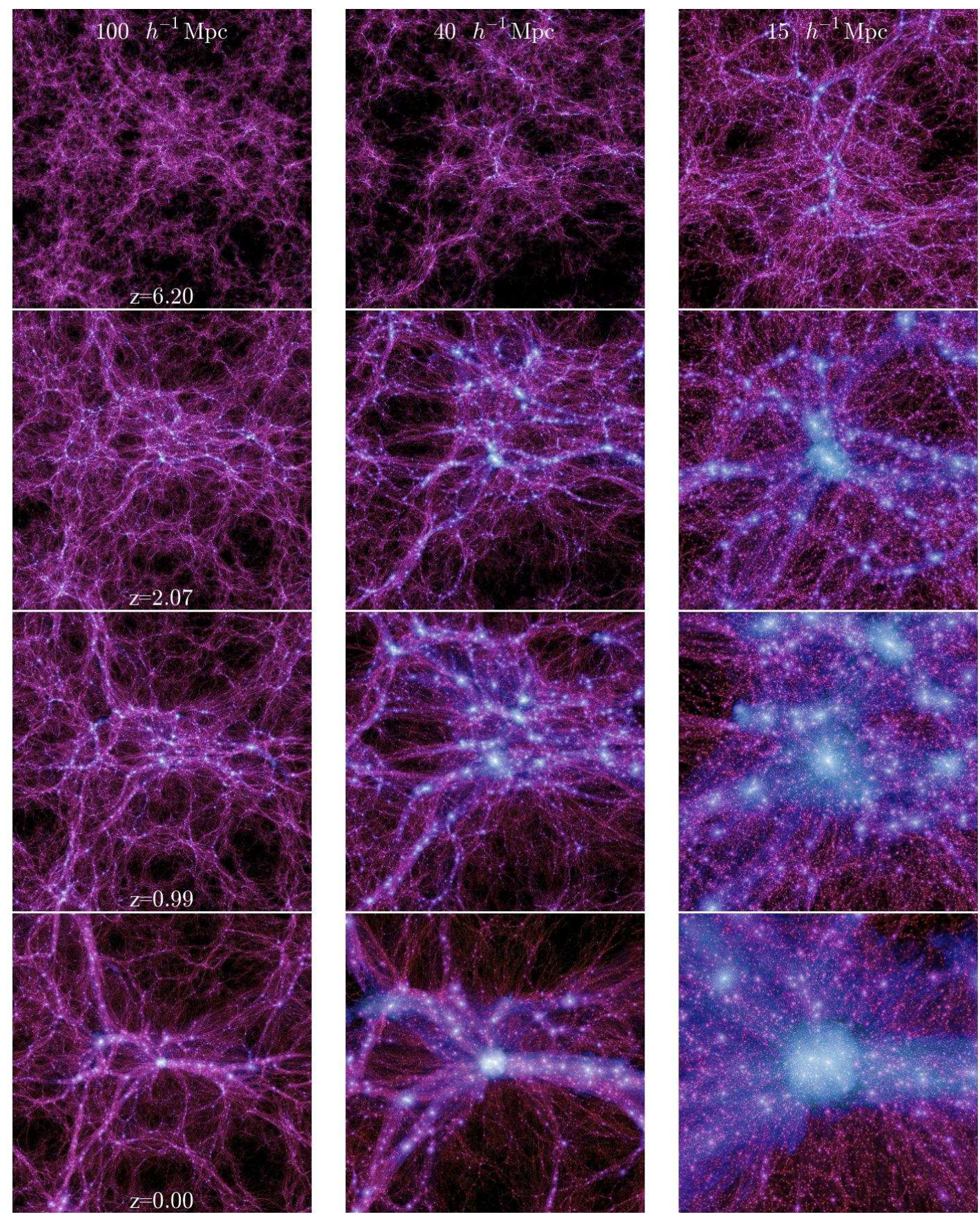

Figura 2.3: Salidas de la simulación Millenium II a diferentes redshifts, de arriba hacia abajo decreciendo en valor de z, y escalas de tamaño, de izquierda a derecha, $100 \mathrm{Mpc}$, $40 \mathrm{Mpc}$ y $15 \mathrm{Mpc}$. Se pueden apreciar los tres regímenes mencionados en la sección 1.2 , cúmulos, panqueques y filamentos y vacíos 
sobredensidad crece como $\delta(x, t) \propto D(t)$, siendo $D(t)$ la tasa de crecimiento lineal. Para una dada cosmología, que gobierna el comportamiento de $D(t)$, podemos determinar las propiedades del campo de densidad a un dado tiempo con respecto a aquéllas en un tiempo temprano $t_{i}$. Sin embargo, el crecimiento de los HMO no es lineal, sino que luego de un tiempo se vuelve altamente no lineal. Una de las opciones para estudiar la distribución de masas sin necesidad de seguir la evolución dinámica en detalle es el formalismo de picos. En el formalismo de los picos de densidad se realiza una suposición fundamental que consiste en considerar que los máximos en el campo de densidad inicial (picos de densidad), coinciden con el lugar "euleriano" de colapso de los halos de materia oscura. El formalismo parte de un campo de densidad suavizado,

$$
\delta(\mathbf{x} ; R) \equiv \int \delta\left(\mathbf{x}^{\prime}\right) W\left(\mathbf{x}+\mathbf{x}^{\prime} ; R\right) d^{3} \mathbf{x}^{\prime}
$$

donde $W(\mathbf{x} ; R)$ es una función ventana de radio característico R. El campo $\delta(\mathbf{x})$ contiene muchos picos(máximos locales) y valles (mínimos locales). La distribución espacial de los picos puede escribirse formalmente como:

$$
n_{p k}(\mathbf{x})=\sum_{p} \boldsymbol{\delta}^{(D)}\left(\mathbf{x}-\mathbf{x}_{\mathbf{p}}\right)
$$

donde $x_{p}$ son las posiciones de los picos. A partir de estas consideraciones simples y realizando una cantidad considerable de álgebra podemos llegar a expresiones para la densidad numérica de picos media en el campo de densidades y su distribución espacial, todo en función de la altura de los picos. También podemos estudiar como los picos se correlacionan espacialmente y hasta su forma, lo cual arroja que picos de mayor amplitud suelen ser más esféricamente simétricos. Sin embargo, no es posible calcular una función de masa de objetos virializados a partir de este formalismo, ya que está afectado por un problema conocido como 'cloud-in-cloud' (nube dentro de la nube), en el que es imposible identificar una sobredensidad dentro de otra de mayor radio, que convive en el campo de densidad cósmico. Para calcular las funciones de masas y las historias de agregación de los halos de MO se utiliza otro tipo de formalismo, menos riguroso y no demostrado, pero que funciona, conocido como formalismo de Press-Schechter (PS). Más tarde, con el desarrollo teórico de "random walks" detallado en Bond et al. (1991), se obtuvo una mejor justificación teórica. 


\section{Estructura interna de halos de MO}

Las simulaciones en computadoras permiten seguir la evolución no lineal de las perturbaciones en el campo de densidades, a partir de condiciones iniciales cosmológicas bien restringidas y realistas. Las estructuras finales de equilibrio cuasi-virial son los HMO que se observa que albergan las galaxias y cúmulos de galaxias. Dubinski \& Carlberg (1991) realizaron la primer simulación de HMO individuales con un número de partículas y resolución suficientes para estudiar su estructura interna (perfiles de densidad y forma). Les llevó un año entero correr en esa época un conjunto de simulaciones con una cantidad de $3 \times 10^{4}$ hasta $3 \times 10^{5}$ partículas.

Desde un punto de vista teórico y considerando un colapso esférico, ya que los HMO en principio tienen simetría esférica, uno esperaría un perfil de densidades de esfera isoterma truncado de la forma $\rho(r) \propto r^{-2}$ para $r<r_{h}$, donde $r_{h}$ es el radio límite del HMO, dentro del cual la densidad media es $\rho_{h}=\Delta_{h} \rho_{c r i t} \Omega_{m}$. Sin embargo, varios efectos modifican este perfil esperado. Por ejemplo, el colapso puede no llegar a un estado de equilibrio en las regiones exteriores del halo. También puede suceder que existan movimientos no radiales y/o que las fusiones asociadas al modelo jerárquico pueden volver al modelo de colapso esférico inviable. Es por esto que se utilizaron simulaciones numéricas para tratar de ajustar el perfil más adecuado para la densidad radial de los HMO.

Navarro et al. (1995) propusieron un perfil simple de densidad radial (perfil de NFW) para los HMO correspondientes a cúmulos que emitían en rayos $\mathrm{X}$,

$$
\rho_{\mathrm{NFW}}(r)=\frac{\rho_{\text {char }}}{\left(r / r_{S}\right)\left(1+r / r_{S}\right)^{2}}
$$

donde $\rho_{\text {char }}$ es una sobredensidad característica, y $r_{s}$ es el radio de escala. La pendiente logarítmica del perfil de NFW cambia gradualmente de -1 cerca del centro a -3 a radios grandes y sólo se parece al de una esfera isoterma en radios $r \approx r_{S}$. En dos artículos posteriores (Navarro et al., 1996, 1997), los autores se encargan de demostrar que este perfil de densided ajusta muy bien a los datos observados para todas las masas de halos de materia oscura y en todas las cosmogonías CDM. De esta manera quedó mostrado que los halos formados por agregación jerárquica sin disipación parecen tener un perfil de densidad universal. La masa encerrada del perfil de NFW está dada por 


$$
M(r)=4 \pi \bar{\rho} \delta_{\text {char }} r_{s}^{3}\left[\ln (1+c x)-\frac{c x}{1+c x}\right],
$$

donde $x=r / r_{h}$ y $c=r_{h} / r_{s}$ es el parámetro de concentración del halo. La masa total se calcula igualando $\mathrm{x}=1$, con lo cual la masa depende de la definición de $r_{h}$. Combinando las ecuaciones 2.23 y 2.24 se puede obtener una relación entre la sobredensidad característica y el parámetro de concentración,

$$
\delta_{\text {char }}=\frac{\Delta_{h}}{3} \frac{c^{3}}{\ln (1+c)-c /(1+c)} .
$$

Así, para una dada cosmología, el perfil de NFW está completamente caracterizado por su masa, $M$, y su parámetro de concentración, $c$, o equivalentemente, por $r_{s}$ y $\delta_{\text {char }}$. Navarro et al. (1997) mostraron que existía una relación entre tiempo de formación del halo y la sobredensidad característica. Los halos que se forman antes son más concentrados. Varios trabajos en los años siguientes estudiaron con más detalle esta correlación entre concentración e historia de formación (Wechsler et al., 2002; Zhao et al., 2003). Encontraron que los halos que sufren una fusión mayor recientemente, típicamente tienen bajas concentraciones $(c \approx 4)$, mientras que los halos que tuvieron un período de crecimiento tranquilo prolongado tienen mayores concentraciones. El escenario actual indica que lo que ocurre físicamente es lo siguiente. La estructura central de los HMO parece ser establecida a través de relajación violenta durante una fase de fusiones rápidas, que conduce a un perfil de NFW universal con $c \approx 4$. Las fusiones posteriores incrementan la masa y el tamaño del halo sin sumar mucho material a sus regiones centrales, incrementando así $r_{h}$ y dejando $r_{s}$ casi sin modificar. Una vez que el especro inicial de potencias y la cosmología son elegidas, la historia de acreción de los halos depende principalmente de su masa relativa a la masa característica $M^{*}$. En particular los halos más masivos se ensamblan más tarde y por lo tanto tienen su última fusión mayor más recientemente. Consecuentemente, la concentración debería ser una función decreciente de la masa de halo. Esto fue visto en las simulaciones de $\mathrm{N}$-cuerpos (Navarro et al., 1997; Bullock et al., 2001). Se observa también que para una dada masa de halo, hay una dispersión considerable en concentraciones, que puede ser modelada por una distribución log-normal con una varianza de aproximadamente $\sigma \approx 0,12 \pm 0,02$ (e.g. Jing, 2000; Wechsler et al., 2002). En el trabajo de Correa et al. (2014), se muestra analíti- 
camente que que la dispersión en la concentración está correlacionada con el tiempo de formación de los halos y que la historia de acreción de masa de los halos se puede relacionar directamente con la tasa de crecimiento de las fluctuaciones iniciales.

Aunque el perfil de NFW es ampliamente utilizado, simulaciones relativamente recientes de alta resolución (Navarro et al., 2004; Hayashi \& White, 2008; Springel et al., 2008) mostraron que los perfiles de densidad promediados esféricamente de halos de materia oscura muestran pequeñas desviaciones del perfil de NFW, y son en promedio, mejor descriptos por un perfil de Einasto (1965):

$$
\rho(r)=\rho_{-2} \exp \left[\frac{-2}{\alpha}\left\{\left(\frac{r}{r_{-2}}\right)^{\alpha}-1\right\},\right]
$$

con $r_{-2}$ el radio al cual la pendiente logarítmica de la distribución de densidad es igual a -2 y $\rho_{-2}=\rho\left(r_{-2}\right)$. El valor de mejor ajuste para el índice $\alpha$ típicamente se mueve en el intervalo $0,12<\alpha<0,25$ y se incrementa sistemáticamente mientras crece la masa (Hayashi \& White, 2008). Si bien el perfil de Einasto tiene 3 parámetros libres y, por lo tanto, es natural que sea más fácil de ajustar en diferentes HMO, en realidad los ajustes resultantes son mejores que los obtenidos con el perfil de NFW aún cuando se fija el valor de uno de los parámetros $(\alpha=0,17)$. El hecho de que $\alpha$ varíe sistemáticamente con la masa demuestra que existe una pequeña desviación de cualquier perfil de densidad de un perfil universal. Un detalle notable del perfil de Einasto es que su pendiente logarítmica es una ley de potencia con dependencia del radio:

$$
\frac{\mathrm{d} \ln \rho}{\mathrm{d} \ln r}=-2\left(\frac{r}{r_{-2}}\right)^{\alpha} .
$$

Entonces, contrario al perfil de NFW, que tiene un pico central $r^{-1}$, la pendiente logarítmica del perfil de Einasto se vuelve cada vez más plana cuando $r$ tiende a 0 . Sin embargo, el perfil de Einasto puede llegar a ser visto como un perfil picudo de todos modos, ya que el radio para el cual $d \ln \rho / d \ln r=-0,5$ es en general menor que $10^{-3} r_{-2}$. En relación a este tema hoy en dia existe todavía una gran controversia sobre la forma del perfil de densidad en radios chicos. A esta discrepancia se la suele conocer como el problema "core-cusp". Las simulaciones cosmológicas indican que en las regiones más densas del Universo, los halos de materia oscura deberían incrementar su densidad hacia el centro, formando un pico (cusp) de densidad. Sin embargo, modelos dinámicos 
obtenidos a partir de observaciones en las regiones centrales de galaxias indican que la densidad de los HMO no parece tener esa forma picuda, sino que muchos autores señalan que los halos tienen cerca del centro un núcleo (core).

Cabe señalar que a pesar del éxito del modelo de concordancia de materia oscura fría con constante cosmológica $\Lambda \mathrm{CDM}$, la distribución de $\mathrm{MO}$ en las galaxias no se deriva de primeros principios, sino que son ajustes obtenidos de simulaciones de partículas de materia oscura solamente, con lo cual la discusión respecto a este aspecto de la teoría no está zanjada. Una de los efectos mencionados como posibles distribuidores de la materia oscura fría, que "desarmaría" el pico de densidad, transformando el pico en un núcleo es el feedback de SN y núcleo galáctico activo (Ogiya \& Mori, 2014). Otro modelo que modifica la forma del HMO es la contracción adiabática por la formación de un disco de materia bariónica en el centro del halo (Blumenthal et al., 1986; Abadi et al., 2010).

Hay muchos aspectos a analizar en la formación de HMO que quedan sin abordar en el presente capítulo, desde las subestructuras en los halos de MO, hasta el estudio de su forma, y quizás más importante, la naturaleza de las partículas de MO. La incertidumbre en este último punto hace que el nivel de especulación sea grande actualmente. El material bibliográfico es extenso y a veces divergente en cuanto a las conclusiones. Abarcar el abanico de teorías y modelos diferentes que existen en la actualidad para explicar la naturaleza de las partículas sería el material para un trabajo en sí mismo.

\subsection{Simulaciones cosmológicas de $N$-cuerpos}

El estudio de la formación y evolución de las galaxias debe realizarse en un contexto cosmológico. Es imposible escindir la evolución de la estructura a gran escala en el Universo de la formación de las galaxias individuales, ya que las galaxias se forman y residen dentro de halos de materia oscura. Hoy en día los acercamientos más comunes al problema son las simulaciones hidrodinámicas auto-consistentes de materia oscura y gas y el modelado semianalítico de formación de galaxias. En el primer caso, la evolución de la materia oscura es trazada dinámicamente, mientras que el gas es tratado

o bien como partículas que interactúan de acuerdo a los principios hidrodinámicos, o bien a través del método Euleriano, en el que se considera una malla de celdas donde se evalúan las propiedades del fluido en el tiempo. Luego, se aplican recetas para distintos 
procesos físicos, como la formación estelar, retroalimentación (o feedback) de supernovas (SNs). En el caso de los modelos semianalíticos se utilizan datos de árboles de fusión ${ }^{6}$ y catálogos de halos de MO como partida y luego se aplican recetas "simples", aunque físicamente motivadas, para modelar la componente bariónica de las galaxias. Los catálogos de halos pueden ser obtenidos analíticamente, a través del formalismo de Press-Schecter extendido o, gracias a los avances en el campo computacional, utilizando simulaciones de $N$-cuerpos que dejan como resultado un catálogo de subhalos de MO que sirve de base para la aplicación del modelo semianalítico de formación y evolución de galaxias. Las ideas originales de este método de estudio fueron concebidas por White \& Rees (1978a), y dieron lugar a un campo de investigación que se fue refinando a lo largo de los años por el trabajo de varios grupos (Cole, 1991; White \& Frenk, 1991; Somerville \& Primack, 1999; De Lucia et al., 2006; Cora, 2006; Guo et al., 2011, por nombrar sólo algunos).

Como fue mencionado, existen varias opciones a la hora de obtener un catálogo de HMO para estudiar la formación y evolución de las galaxias que se forman en su interior. Los métodos analíticos como el formalismo de PSE descripto en este capítulo se presentan como una solución accesible. Otro método utilizado para construir catálogos de HMO son las simulaciones de $\mathrm{N}$-cuerpos, mediante las cuales se realizan simulaciones dinámicas de partículas de materia oscura de una masa mínima dada, a partir de condiciones inciales cosmológicas. Sin embargo ambos métodos no arrojan resultados idénticos. Un resultado directo de estos catálogos es la función de masa de HMO, esto es, el número de HMO por intervalo de masa. El método de PSE predice un número excesivo de HMO de masas menores a la masa característica $M^{\star}$ y subestima la cantidad de HMO de masas mayores a ésta (Somerville et al., 2001). Los modelos basados en refinamientos del mismo (e.g. Sheth \& Tormen, 1999) disminuyen la magnitud de este comportamiento, pero siguen reproduciéndolo. Además, ha sido demostrado que los árboles de fusión obtenidos de métodos tipo EPS predicen tiempos de formación que son sistemáticamente más largos que aquellos obtenidos de simulaciones de $\mathrm{N}$-cuerpos (Wechsler et al., 2002).

En este trabajo de tesis se utiliza una técnica numérica basada en la combinación de una simulación cosmológica de $\mathrm{N}$-cuerpos de $\mathrm{MO}$ y un modelo semianalítico de forma-

\footnotetext{
${ }^{6} \mathrm{Se}$ define como un árbol de fusiones a la historia de fusiones sufridas por un halo de materia oscura y sus progenitores
} 
ción y evolución de galaxias con el cual simulamos los procesos bariónicos involucrados en la formación y evolución de las galaxias. Las características de la simulación se detallan a continuación, mientras que el Capítulo 3 está dedicado a la descripción del modelo semianalítico.

\section{Simulación cosmológica de $N$-cuerpos Stand}

A lo largo del presente trabajo se hace uso de las salidas de una simulación de $\mathrm{N}$ cuerpos corrida en el cluster de computo Geryon dispuesto por el instituto de Astrofísica de la Pontificia Universidad Católica de Chile; la misma fue denominada Stand. En lo que sigue describiremos las características principales de la simulación. La simulación se corrió con condiciones iniciales acordes al escenario $\Lambda \mathrm{CDM}$ y fue caracterizada con los parámetros cosmológicos $\Omega_{\mathrm{m}}=0,28, \Omega_{\mathrm{b}}=0,046, \Omega_{\Lambda}=0,72, h=0,7, n=0,96$, $\sigma_{8}=0,82$, de acuerdo a la cosmología WMAP7 (Jarosik et al., 2011). Si bien estos parámetros difieren de los últimos parámetros obtenidos por Planck Collaboration et al. (2014), un trabajo de Guo et al. (2013) mostró que no existen diferencias notables en los resultados de un modelo semianalítico de formación de galaxias cuando se utilizan los parámetros de WMAP1 y WMAP7 en la simulación subyacente. Consideramos que pequeñas variaciones en los parámetros cosmológicos no traen grandes cambios en los resultados del modelo semianalítico. Nuestra simulación fue corrida con el código de $N$-cuerpos GADGET-2 (Springel, 2005) usando $640^{3}$ partículas en una caja cúbica de longitud de lado comóvil de $L=150 \mathrm{~h}^{-1} \mathrm{Mpc}$. Las condiciones iniciales fueron generadas usando GRAFIC2 (Bertschinger, 2001). La simulación fue evolucionada desde $z_{\text {ini }}=61,2$ a la época presente, guardando 100 salidas igualmente espaciadas en $\log _{10}(a)$ entre $z=20$ y $z=0$ (Benson et al., 2012).

Los halos de DM fueron identificados usando el algoritmo friends-of-friends (FOF). Luego, la aplicación del algoritmo SUBFIND (Springel et al., 2001) permite la selección de subestructuras ligadas (sub-halos) que tienen al menos 10 partículas con $1 \times$ $10^{9} h^{-1} \mathrm{M}_{\odot}$ cada una. Los halos de DM y los subhalos tienen masas en el intervalo $1 \times 10^{10}-7,8 \times 10^{14} h^{-1} \mathrm{M}_{\odot}$. La partícula más ligada en cada subhalo es usada por el modelo semianalítico para trazar las posiciones y velocidades de las galaxias dentro de un halo FOF. 


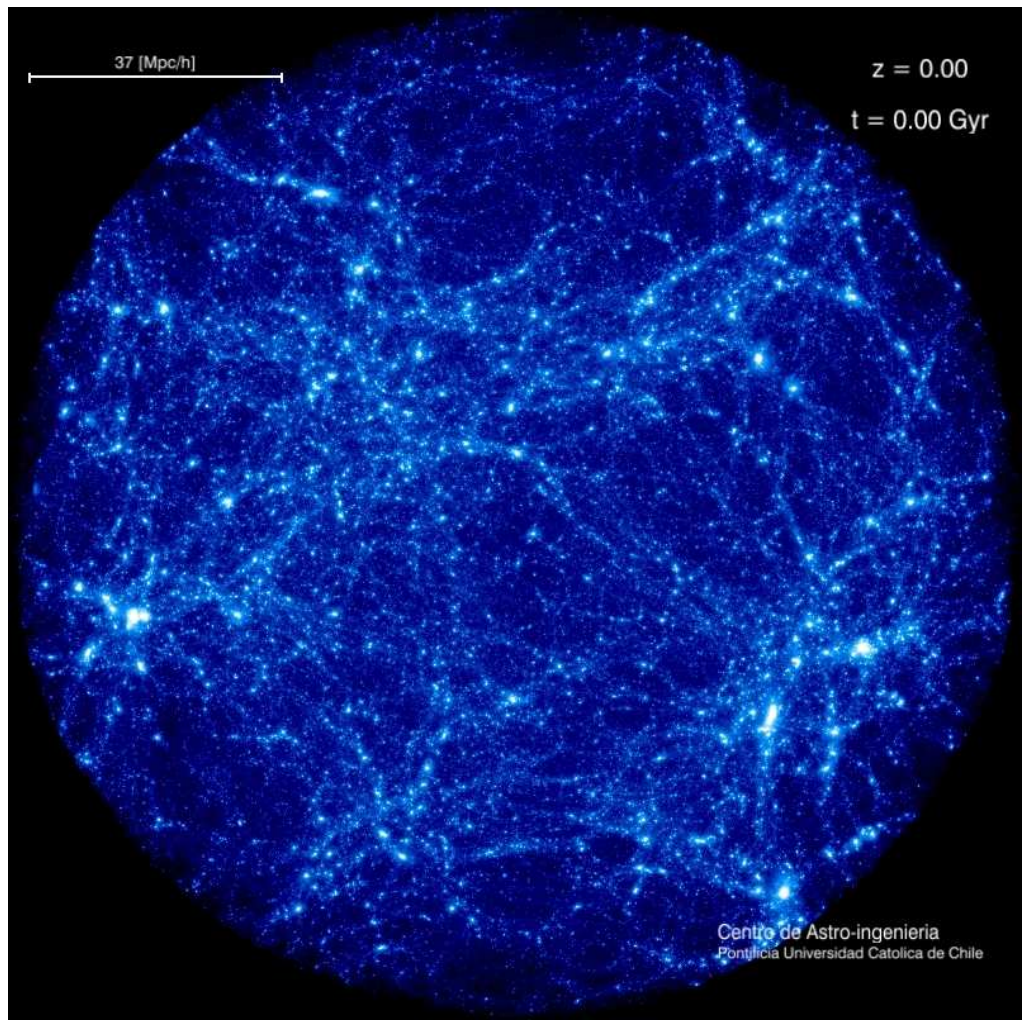

Figura 2.4: Imagen de un recorte esférico de la caja de la simulación Stand utilizada durante el desarrollo de esta tesis. Los créditos de la imagen corresponden a Álvaro Orsi, miembro del grupo de investigación. 



\section{Capítulo 3}

\section{Modelo semianalítico de formación y evolución de galaxias: SAG}

El modelo semianalítico utilizado para llevar a cabo la presente tesis es llamado SAG (acrónimo de Semi-Analitic Galaxies). El mismo se basa en la versión descrita por Cora (2006, C06 de ahora en adelante). Posteriormente fue mejorada mediante la inclusión del efecto de retroalimentación de núcleos galácticos activos y de inestabilidades de disco (Lagos et al., 2008a) y con la inclusión de efectos de ambiente como la presión de barrido (ram pressure) y una refinación en el modelado del tamaño de los discos galácticos (Tecce et al., 2010). Sobre la base de este código, en los últimos años se han desarrollado nuevas herramientas para el análisis de las poblaciones de galaxias simuladas y se han incluido nuevas recetas para describir los procesos involucrados en la formación y evolución de las galaxias. Orsi et al. (2014) agregan el modelado de líneas de emisión del gas en galaxias. Padilla et al. (2013) consideran el criterio de inestabilidad de disco para establecer la presencia de brotes estelares en una galaxia remanente luego de una fusión ${ }^{1}$; esta condición está inspirada en el hecho de que el material acretado sobre el disco galáctico durante una fusión tiene momento angular desalineado debido a los cambios repentinos de momento angular de los halos de $\mathrm{MO}$ que contienen a las galaxias que se fusionan, lo cual inhibe el crecimiento del momento angular del disco de la galaxia remanente. Finalmente, Gargiulo et al. (2014) incluyen la posibilidad de elegir

\footnotetext{
${ }^{1}$ El modelo utilizado en este trabajo no tiene en cuenta este tratamiento; el criterio que define la existencia de brotes estelares durante una fusíon depende de la relación de masas de las galaxias que intervienen y de sus respectivos contenidos de gas frío.
} 
diferentes funciones iniciales de masa estelar y una receta para simular los brotes de formación estelar extendidos en el tiempo. Esto mejora la suposición original presente en el modelo, en la que los brotes de formación ocurrían de manera instantánea. Además, los productos de la nucleosíntesis estelar (yields estelares) fueron actualizados. Los últimos avances mencionados permitieron estudiar en detalle y más consistentemente las propiedades químicas de las galaxias y sus componentes, y son parte del presente trabajo de tesis. De esta manera, se dispone de un código que permite recuperar numerosas relaciones observacionales que involucran las masas, colores y metalicidades de las galaxias y sus componentes, constituyendo una excelente base para llevar a cabo la investigación propuesta. El buen acuerdo con las observaciones se logra mediante la calibración del modelo, que resulta de ajustar los parámetros libres involucrados en cada uno de los procesos físicos considerados de modo que las propiedades de las galaxias modeladas reproduzcan un cierto conjunto de propiedades observadas. Estos procesos son modelados mediante ecuaciones diferenciales simples, las cuales son resueltas en pasos de tiempo de tamaño $\Delta t=\Delta T / N_{\text {steps }}$, donde $\Delta T$ es el intervalo de tiempo entre salidas consecutivas de la simulación subyacente, y $N_{\text {steps }}=25$. Si bien el modelo SAG ajusta razonablemente un amplio rango de estadísticas observacionales, éste no es el objetivo último de un modelo semianalítico de formación de galaxias, si no el de investigar el efecto de nuevos procesos (o distintos procesos) sobre las propiedades de las galaxias. El presente trabajo de tesis se enfoca principalmente en investigar el impacto de diferentes funciones iniciales de masa estelar, incluyendo en el análisis otro tipo de estadísticas que incluyen propiedades fotométricas y químicas de las poblaciones estelares.

En este capítulo se presentan las recetas incluidas en el código SAG para modelar cada proceso físico involucrado en la formación y evolución de las galaxias, y mostrar resultados de las calibraciones llevadas a cabo para realizar las investigaciones detalladas en los siguientes capítulos.

\section{Tipos de galaxias y fusiones}

De acuerdo al tratamiento tradicional hecho en modelos semianalticos, el mayor subhalo de un grupo FOF alberga la galaxia central del grupo, y su posición está dada por la de la partícula más ligada de ese subhalo. Las galaxias centrales de otros subhalos menores contenidos en el grupo FOF son galaxias satélites con subhalo y nos referimos 
a ellas como galaxias tipo 1. Los subhalos de estas galaxias satélites se mantienen intactos luego de caer en estructuras mayores. El tercer grupo de galaxias comprende a las galaxias satélites huérfanas que provienen de la fusión de dos subhalos, donde la galaxia residente en el subhalo menor se vuelve satélite de la galaxia central residente en el subhalo remanente; las mismas son denominadas galaxias satlites tipo 2. En el modelo semianalítico, se supone una órbita circular para estas galaxias satélites que han perdido su subestructura, con una velocidad y distancia radial inicial dadas, respectivamente, por la velocidad y radio viriales del subhalo remanente al que pertenecen. Estas galaxias se fusionan con la galaxia central de su subhalo en una escala de tiempo definida por el proceso de fricción dinámica (Binney \& Tremaine 1987),

$$
T_{\mathrm{df}}=\alpha \frac{f(\varepsilon)}{2 \mathrm{C}} \frac{V_{\mathrm{C}} r_{\mathrm{c}}^{2}}{\mathrm{G} \ln \Lambda M_{\mathrm{sat}}}
$$

donde $M_{\text {sat }}$ es la masa del satélite orbitando a un radio $r_{\mathrm{c}}$ dentro de un halo isotérmo de velocidad circular $V_{\mathrm{C}}$, y $\alpha=1,17$. La función $f(\varepsilon)$ describe la dependencia con la excentricidad de la órbita del satélite (Springel et al. 2001) y $C=0,43 . \operatorname{Ln}(\Lambda)$ es el logaritmo Coulombiano.

Teniendo definidas estas tres clases de galaxias, se inicializa la población con un conjunto de galaxias centrales, una para cada subhalo, con masa de gas frío, masa estelar y luminosidad en cero. Las propiedades físicas de estas galaxias evolucionan según las recetas del código semianalítico que es combinado con la historia de fusiones de los subhalos de materia oscura. Siguiendo este procedimiento de salida en salida de la simulación cosmológica subyacente, se obtiene la población de galaxias a tiempo presente, además de guardarse la información en épocas intermedias. Los procesos físicos que se modelan mediante el código semianalítico son: enfriamiento radiativo de gas caliente transformación de gas frío en estrellas, recalentamiento de gas frío por retroalimentación energética (feedback) de supernovas y AGN, decaimiento orbital de los satélites y la consecuente fusión con las galaxias centrales, inestabilidades de disco, brotes estelares durante los eventos de fusión y de inestabilidades de disco, y evolución química de las distintas componentes bariónicas (gas caliente, gas frío y estrellas). 


\section{Enfriamiento radiativo del gas caliente}

El gas, a diferencia de la MO, es de naturaleza disipativa. Los HMO que son formados a partir de sobredensidades primordiales forman pozos de potencial y atraen en su colapso al material bariónico gaseoso que es calentado por los choques que se producen. La cantidad de gas presente inicialmente es la fracción bariónica cosmológica supuesta en el conjunto de datos cosmológicos utilizados, calculada en función de la masa del halo y se supone que tiene una distribución de esfera isoterma,

$$
\rho_{g}(r)=\frac{M_{\mathrm{hot}}}{4 \pi R_{\mathrm{vir}} r^{2}}
$$

Los HMO crecen a medida que transcurre el tiempo en la simulación por acreción de halos más pequeños y arrastra una fracción de gas igual a la fracción bariónica. En el modelo SAG, la masa de gas acretada es sumada al reservorio de gas caliente, ya que se supone que es calentado por choques. Además, debe tenerse en cuenta la cantidad de gas que se enfría y que luego da lugar a formación estelar, y el crecimiento de los agujeros negros, que son alimentados por material bariónico. Así, el contenido de gas caliente durante de la evolución de la galaxia, se calcula en el modelo como:

$$
M_{\mathrm{hot}}=f_{\mathrm{b}} M_{\mathrm{vir}}-\sum_{\mathrm{i}=1}^{N_{\mathrm{FOF}}}\left(M_{\mathrm{stellar}}+M_{\mathrm{cold}, \mathrm{i}}+M_{\mathrm{BH}, \mathrm{i}}\right) \text {. }
$$

donde $f_{\mathrm{b}}$ es la fracción bariónica, $M_{\text {stellar }}$ la masa de la galaxia en estrellas, $M_{\text {cold }}$ la masa en gas frío y $M_{\mathrm{BH}}$ la masa del agujero negro central de la galaxia. La masa virial está dada por $M_{\mathrm{vir}}=100 H^{2} R_{\mathrm{vir}}^{3} / G$. La suma se realiza para todos los subhalos contenidos en el halo FOF, $N_{\text {FOF. }}$. Es importante notar que esto representa el caso estándar del modelo, en la que a las galaxias satélites (tipo 1 y tipo 2) no retienen su halo de gas caliente al convertirse en satélites, sufriendo un proceso conocido como "estrangulación"(Larson et al., 1980; Balogh et al., 2000). Este proceso es una forma simplificada de tener en cuenta los efectos de ambiente. En una versión mejorada del código esta condición se relaja y, si bien las galaxias satélites pueden perder gas caliente por desprendimiento por efecto de mareas y de presión de barrido, queda disponible gas caliente en las galaxias satélites, que se consume gradualmente a través de enfriamiento radiativo, tal como ocurre en las galaxias centrales. Sin embargo, la versión utilizada en el presente trabajo no considera esta opción, debido a que se encontraba en desarrollo durante la realización 
del mismo.

El gas caliente presente en los HMO llega a temperaturas superiores a $T=10^{4}$ ${ }^{\circ} K$, suficiente para ionizar el gas (White \& Rees, 1978a). Los electrones libres generan radiación de frenado, o Brehmstrahlung. Ésto, sumado a recombinaciones y emisión de líneas por colisiones, produce una tasa de enfriamiento $\Lambda(T, Z) n_{\mathrm{e}} n_{\mathrm{H}}$ que depende tanto de la temperatura como de la metalicidad del gas. Para estimar esta tasa, el modelo SAG utiliza las potencias radiadas por elemento químico publicadas por Foster et al. (2012). La masa de gas frío se ve modificada en el tiempo como:

$$
\frac{\mathrm{d} M_{\text {cool }}}{\mathrm{d} t}=4 \pi \rho_{\mathrm{g}} r_{\mathrm{cool}}^{2} \frac{\mathrm{d} r_{\text {cool }}}{\mathrm{d} t}
$$

donde $\rho_{\mathrm{g}}$ es la densidad del gas y $r_{\text {cool }}$ el radio de enfriamiento, definido como el radio para el cual el tiempo de enfriamiento (dado por el cociente entre el contenido de energía térmica específica del gas y la tasa de enfriamiento por unidad de volumen) es igual al tiempo en que el halo es capaz de enfriarse quasi-estáticamente; este último es aproximado por el tiempo dinámico del halo, $R_{\mathrm{vir}} / V_{\mathrm{vir}}$.

\section{Formación Estelar}

El gas frío disponible en las galaxias a partir del enfriamiento radiativo es consumido a través del proceso de formación estelar. Se supone que el momento angular en la disipación se conserva, formando un disco en el que se produce la formación estelar según una tasa dada por:

$$
\frac{d M_{\star}}{d t}=\alpha \frac{M_{\mathrm{cold}}-M_{\mathrm{cold}, \mathrm{crit}}}{t_{\mathrm{dyn}}},
$$

con

$$
M_{\text {cold }, \text { crit }}=3,8 \times 10^{9}\left(\frac{V_{\text {vir }}}{200 \mathrm{~km} \mathrm{~s}^{-1}}\right)\left(\frac{3 R_{\text {disc }}}{10 \mathrm{kpc}}\right) \mathrm{M}_{\odot},
$$

donde $t_{\text {dyn }}=V_{\text {vir }} / 3 R_{\text {disc }}$ es el tiempo dinámico de la galaxia, $V_{\text {vir la velocidad circular }}$ en el radio virial, y $R_{\text {disc }}$ la longitud de escala de un disco exponencial con el cual se representa la distribución de masa del disco (Tecce et al., 2010). Si la masa de gas frío excede $M_{\text {cold,crit, }}$ ocurre la formación estelar, siguiendo a Croton et al. (2006). Nos referimos a este modo de formación estelar en el disco como modo quiescent de formación 
estelar.

\section{Formación de discos}

El modelo SAG considera la formación de discos galácticos mediante un cálculo detallado que involucra el parámetro de spin $\lambda$ (Peebles, 1969) y sigue los lineamientos generales planteados por Mo et al. (1998). La masa del disco resulta una fracción de la masa del halo anfitrión, el momento angular del disco es una fracción del momento angular del halo, el disco es una estructura delgada en equilibrio de rotación con un perfil de densidad exponencial. Sólo aquellos sistemas dinámicamente estables pueden corresponder a discos galácticos reales. Basándose en estas suposiciones, se generaron tablas con los valores del radio de escala correspondientes a distintos valores de concentraciones de halo de $\mathrm{MO}$, masas de bulbo, masas de disco y parámetros de spin, a las que se accede a través de interpolaciones a medida que corre el modelo, logrando obtener radios de escala para cada galaxia en cada instante de la simulación. Se refiere al lector a Tecce et al. (2010) para más detalles de esta aspecto particular del modelo y de su implementación en el código SAG.

\section{Formación de esferoides: bulbos y galaxias elípticas}

Cuando ocurre una fusión de galaxias, se evalúa el cociente de masas entre la galaxia satélite y la galaxia central $M_{\text {sat }} / M_{\text {cen }}$. Si $M_{\text {sat }} / M_{\text {cen }}>0,3$, entonces la fusión es considerada una fusión mayor. En este caso, todo el gas en la galaxia remanente es consumido en un brote estelar contribuyendo a la formación del bulbo, y el disco estelar se relaja completamente y es transferido al bulbo. El desencadenamiento de un brote de formación estelar en una fusión menor $\left(M_{\mathrm{sat}} / M_{\mathrm{cen}} \leq 0,3\right)$ dependerá de la fracción de gas frío presente en el disco de la galaxia central, tal cual se implementó en Lagos et al. (2008a) siguiendo el trabajo de Malbon et al. (2007). Si el cociente entre el gas frío y la masa de disco de la galaxia central, $M_{\text {cold,cen }} / M_{\text {disc,cen, es mayor que un parámetro fijo }}$ $f_{\text {burst }}=0,6$, entonces la perturbación introducida por el satélite que se fusiona conduce todo el gas frío de ambas galaxias hacia la componente bulbo, donde es consumido en un brote de formación estelar; el disco estelar de cada galaxia es transferido al bulbo de 
la galaxia remanente. Sin embargo, si la galaxia satélite es mucho menos masiva que la central, ( $\left.M_{\mathrm{sat}} / M_{\mathrm{cen}} \leq 0.05\right)$, no ocurre ningún brote. En fusiones menores, sólo las estrellas de los satélites en fusión son transferidas a la componente de bulbo de la galaxia central.

El otro canal que contribuye a la formación de la componente de bulbo es la inestabilidad de disco global. Algunas configuraciones de discos galácticos no permanecen estables con el tiempo. Cuando un disco galáctico es lo suficientemente masivo como para que su propia fuerza de gravedad sea dominante, el disco se vuelve inestable. Esta condición es expresada en el modelo a través del criterio de Efstathiou-Lake-Negroponte (Efstathiou et al., 1982), que dice que la estabilidad a la formación de una barra se pierde cuando

$$
\varepsilon_{\mathrm{d}} \equiv \frac{V_{\mathrm{disc}}}{\left(G M_{\mathrm{disc}} / R_{\mathrm{disc}}\right)^{1 / 2}} \leq \varepsilon_{\mathrm{thresh}}
$$

donde $M_{\text {disc }}$ es la masa del disco (gas frío más estrellas), $R_{\text {disc }}$ es el radio de escala del disco, y $V_{\text {disc }}$ es la velocidad circular del disco. Para expresar la última cantidad, usamos la velocidad a la cual la curva de rotación se vuelve plana, la cual aproximamos con la velocidad calculada a $\sim 3 R_{\text {disc }}$ (ver Tecce et al. (2010) o Sección 4 para detalles concernientes a formación de discos en el modelo SAG). El parámetro libre $\varepsilon_{\text {thresh }}$ tiene valores cercanos a la unidad; en nuestro modelo, adoptamos $\varepsilon_{\text {thresh }}=1$. Consideramos un parámetro libre adicional que tiene en cuenta la influencia de las galaxias que cumplen el rol de perturbar y provocar efectivamente la inestabilidad de disco; esto es modelado calculando la separación media entre galaxias en un grupo FOF. Cuando la separación media es menor que $f_{\text {pert }} R_{\text {disc }}$, siendo $f_{\text {pert }}$ un parámetro libre, consideramos que una galaxia vecina perturba el disco inestable. Como consecuencia de esto, todas las estrellas y el gas frío en ese disco inestable son transferidas a la componente de bulbo, $\mathrm{y}$ todo el gas presente es consumido en un brote estelar.

La clasificación morfológica de galaxias generadas por el modelo está basada en el cociente entre la luminosidad en la banda- $r$ del bulbo y la total, B/T. Las galaxias elípticas son aquéllas caracterizadas por un cociente $\mathrm{B} / \mathrm{T}>(\mathrm{B} / \mathrm{T})_{\text {thresh }}$, donde el parámetro $(\mathrm{B} / \mathrm{T})_{\text {thresh }}$ puede adoptar valores en el intervalo $\sim 0,7-0,85$. Así, la formación de galaxias elípticas está directamente conectada con la formación de bulbos, la cual en SAG ocurre a través de inestabilidades de disco globales y los dos tipos de fusiones de 
galaxias mencionados.

\section{Retroalimentación por supernovas}

La formación de estrellas provocada por el enfriamiento del gas genera que una parte de ese gas vuelva a ser recalentado. Esto ocurre porque la fracción de estrellas que terminan sus vidas como $\mathrm{SN}$ de colapso de núcleo, de masa inicial mayor a $8 M_{\odot}$, como las SNII o tipo Ib/c, inyectan energía en el medio a partir de su explosión. La cantidad de gas frío recalentado producido por un evento de formación estelar que genera una población estelar de masa $\Delta M_{\star}$ es:

$$
\Delta M_{\text {reheated }}=\frac{4}{3} \varepsilon \frac{\eta E}{V_{\text {vir }}^{2}} \Delta M_{\star}
$$

donde $E=10^{51} \mathrm{erg} \mathrm{s}^{-1}$ es la energía liberada por una SN, y $\eta$ es el número de SNs que depende de la función IMF adoptada, y se estima como

$$
\eta=\frac{\int_{8}^{\infty} \phi(m) m \mathrm{~d} m}{\int_{0}^{\infty} \phi(m) \mathrm{d} m},
$$

donde $\phi(m)$ es la IMF adoptada y $m$ la masa estelar. Esta cantidad es constante para una IMF de Salpeter, por ejemplo, y varía de acuerdo a la pendiente adoptada por una IMF variable. A partir de este trabajo de tesis se modificó en el modelo SAG el tratamiento de retroalimentación para el caso de los bulbos y los discos, que se tratan ahora como componentes separadas. La eficiencia $\varepsilon$ de la ec. 3.8 corresponde a estrellas nacidas en el disco. Un nuevo parámetro es introducido para regular la retroalimentación en el bulbo, al cual nos referimos como $\varepsilon_{\text {bulge }}$. En ambos casos la cantidad de masa recalentada está dada por la ecuación 3.8, pero con diferentes valores de eficiencia según la calibración adoptada.

\section{Retroalimentación por núcleos de galaxias activos}

Existe una estrecha relación entre los núcleos de galaxias activos (AGN, por las siglas en inglés de Active Galactic Nuclei ) y la evolución de las galaxias en las que habitan. Una correlación típica que se evidencia es aquella entre la masa del agujero 
negro central, y la masa del bulbo (Häring \& Rix, 2004) . Los jets producidos por AGN son capaces de transportar grandes cantidades de energía al gas que rodea a la galaxia, regulando en gran medida la cantidad de gas que se enfría y disminuyendo la formación estelar de la galaxia que los alberga. En general, esto ocurre para las galaxias más masivas (Lagos et al., 2008b, LCP08). En el modelo SAG, el crecimiento del agujero negro tiene lugar a través de dos mecanismos diferentes. Por un lado, la caída de gas hacia el centro galáctico por eventos de fusión o inestabilidad de disco hace crecer al agujero negro. Este modo de crecimiento es llamado modo quasar y la masa de gas acretada por el AGN está dada por:

$$
\Delta M_{\mathrm{BH}}=f_{\mathrm{BH}} \frac{M_{\text {sat }}}{M_{\text {cen }}} \frac{M_{\text {cold,sat }}+M_{\text {cold,cen }}}{\left(1+280 \mathrm{~km} \mathrm{~s}^{-1} / V_{\text {vir }}\right)^{2}}
$$

donde $f_{\mathrm{BH}}$ es la fracción de gas frío del remanente de la fusión, $M_{\mathrm{cen}} \mathrm{y} M_{\mathrm{sat}}$ son las masas de la galaxia central y satélite que se fusionan, y $M_{\text {cold,cen } \mathrm{y}} M_{\text {cold,sat }}$ sus correspondientes masas de gas frío. Cuando se trata de una inestabilidad de disco, sólo está involucrado el gas frío de la galaxia inestable. En segundo lugar, los agujeros negros crecen por la acreción de gas caliente durante la fase de enfriamiento del gas que ocurre en las galaxias centrales como:

$$
\frac{d M_{\mathrm{BH}}}{d t}=\kappa_{\mathrm{AGN}} \frac{M_{\mathrm{BH}}}{10^{8} \mathrm{M}_{\odot}} \frac{f_{\mathrm{hot}}}{0,1}\left(\frac{V_{\mathrm{vir}}}{200 \mathrm{~km} \mathrm{~s}^{-1}}\right)^{2}
$$

donde $f_{\text {hot }}=M_{\text {hot }} / M_{\text {vir }}$. El parámetro libre $\kappa_{\mathrm{AGN}}$ es la eficiencia de la acreción. Este modo de crecimiento es conocido como el modo de radio. Para mayor detalle de esta implementación en el modelo SAG se refiere al lector a Lagos et al. (2008b).

\section{Incorporaciones al modelo}

La investigación desarrollada en el trabajo de tesis requirió la incorporación de recetas nuevas en el modelado semianalítico. Las nuevas implementaciones, como los brotes estelares extendidos o una nueva función inicial de masa estelar variable, resultan necesarias para estudiar de manera más consistente los procesos físicos involucrados en el enriquecimiento químico de las galaxias. En esta sección se describe la implementación de estas modificaciones y de algunos aspectos ya incorporados previamente, pero que 
necesitan ser explicados en detalle para una mejor comprensión de los resultados.

\subsection{Modelado químico, nuevos yields}

La componente gaseosa primordial en el modelo tiene una abundancia inicial de $76 \%$ de hidrógeno y $24 \%$ de helio, siguiendo los valores estándar de la nucleosíntesis primordial y obviando el litio, ya que no es individualizado en el modelado. En nuestro modelo de enriquecimiento químico, seguimos la producción de diez elementos químicos $\left(\mathrm{H},{ }^{4} \mathrm{He},{ }^{12} \mathrm{C},{ }^{14} \mathrm{~N},{ }^{16} \mathrm{O},{ }^{20} \mathrm{Ne},{ }^{24} \mathrm{Mg},{ }^{28} \mathrm{Si},{ }^{32} \mathrm{~S},{ }^{40} \mathrm{Ca}\right)$ generados por estrellas en diferentes intervalos de masa, desde estrellas de masa baja e intermedia a estrellas quasi-masivas y masivas. La versión del modelo SAG utilizada para el estudio del presente trabajo está caracterizada por un nuevo conjunto de yields estelares. Usamos la mejor combinación de yields comunicada por Romano et al. (2010), seleccionada para estar en acuerdo con un gran número de restricciones observacionales conocidas para la Vía Láctea. Para estrellas de masa baja e intermedia, en el intervalo de masa $1-8 \mathrm{M}_{\odot}$, usamos los yields de Karakas (2010). Para la pérdida de masa de estrellas pre-supernova (He and CNO elements), usamos los yields calculados por el grupo de Génova (Hirschi et al., 2005), y para los yields de nucleosíntesis explosiva debido a SN II, usamos los resultados de Kobayashi et al. (2006). En todos los casos adoptamos la masa eyectada total de modelos de estrellas con metalicidad solar, para los cuales la composición solar se supone que es $Z_{\odot}=0,02$, y las abundancias iniciales son tomadas de Anders \& Grevesse (1989).

Las eyecciones de SN Ia también son incluidas. Éstas están caracterizadas por una alta producción de hierro, de alrededor de $\sim 0,6 \mathrm{M}_{\odot}$. Consideramos las recetas de nucleosíntesis del modelo W7 actualizado por Iwamoto et al. (1999). Las tasas de SNIa son estimadas usando el modelo de degeneración simple, en el cual una SNIa ocurre por deflagración del carbono en enanas blancas de C-O, en sistemas binarios cuyas componentes tienen masas entre 0,8 y $8 \mathrm{M}_{\odot}$ (Greggio \& Renzini, 1983); implementamos el formalismo presentado por Lia et al. (2002) (ver su sección 4.2.2). La fracción de estos sistemas binarios, $A_{\text {bin, }}$ es uno de los parámetros libres de SAG. Tomamos en cuenta la escala de tiempo de retorno de pérdida de masa y eyecciones de todas las fuentes consideradas. Para este propósito, usamos los tiempos de vida estelares dados por Padovani \& Matteucci (1993), que usa resultados de Matteucci \& Greggio (1986) para masas sobre 
6,6 $\mathrm{M}_{\odot} \mathrm{y}$ de Renzini \& Buzzoni (1986) para masas por debajo de este límite. Este aspecto se vuelve especialmente relevante para este trabajo debido a los diferentes tiempos de retardo que caracterizan a diferentes tipos de SNs, los cuales afectan a la abundancia relativa de elementos $\alpha$ relativa al hierro, tema central en los capítulos siguientes. Para una descripción detallada de la implementación en SAG del enriquecimiento químico de las diferentes componentes bariónicas, referimos al lector a Cora (2006).

\subsection{Una nueva teoría de IMF Variable}

La IMF estelar tiene un gran impacto en el enriquecimiento químico de las galaxias, ya que define la cantidad de estrellas formadas en cada intervalo de masa para cada evento de formación estelar $\mathrm{y}$, consecuentemente, impacta en la cantidad de metales devueltos al medio interestelar, donde nuevas estrellas serán formadas eventualmente. La IMF también determina el número de SNII involucradas en la estimación de la masa recalentada que es transferida de la fase fría a la fase caliente durante el proceso de retroalimentación de SN. Hoy en día existe algún grado de consenso en que en una población estelar simple la pendiente de la IMF para masas estelares mayores que $1 \mathrm{M}_{\odot}$ no es estrictamente diferente de la IMF de Salpeter $(\alpha \equiv 1+x=2,35)$; estrellas con masas por encima de $1 \mathrm{M}_{\odot}$ son las mayores contribuyentes al enriquecimiento químico (Chiosi et al., 1998). Varios intentos se han realizado para medir esta importante función de distribución en la vecindad solar (Salpeter, 1955; Chabrier, 2003) o directamente en cúmulos estelares (Kroupa, 2001; Kroupa \& Boily, 2002). Esta última está representada por una IMF tipo ley de potencias multi-componente dado por

$$
\xi(m)=k \begin{cases}k^{\prime}\left(\frac{m}{m_{\mathrm{H}}}\right)^{-\alpha_{0}} & , m_{\mathrm{low}} \leq m / M_{\odot}<m_{\mathrm{H}} \\ \left(\frac{m}{m_{\mathrm{H}}}\right)^{-\alpha_{1}} & , m_{\mathrm{H}} \leq m / M_{\odot}<m_{0}, \\ \left(\frac{m_{0}}{m_{\mathrm{H}}}\right)^{-\alpha_{1}}\left(\frac{m}{m_{0}}\right)^{-\alpha_{2}} & , m_{0} \leq m / M_{\odot}<m_{1}, \\ \left(\frac{m_{0}}{m_{\mathrm{H}}}\right)^{-\alpha_{1}}\left(\frac{m_{1}}{m_{0}}\right)^{-\alpha_{2}}\left(\frac{m}{m_{1}}\right)^{-\alpha_{3}} & , m_{1} \leq m / M_{\odot} \leq m_{\max },\end{cases}
$$

con $m_{\text {low }}=0,01, m_{\mathrm{H}}=0,08, m_{0}=0,5 \mathrm{y} m_{1}=1,0$, y exponentes $\alpha_{0}=0,3, \alpha_{1}=1,3$, $\alpha_{2}=\alpha_{3}=2,35$. La constante de normalización $k$ contiene la normalización elegida para la IMF (por ejemplo, en función de la masa total del sistema). Notar que las enanas 
marrones son una población separada y contribuyen algo así como el 1.5 por ciento de la masa sólamente. Por eso, es necesario un factor de normalización diferente, $k^{\prime}$, en la IMF $\left(k^{\prime} \sim 1 / 3\right.$, Thies \& Kroupa, 2007, 2008).

En el caso de sistemas estelares con poblaciones estelares compuestas, otros aspectos deben ser considerados. Una IMF de toda la galaxia puede ser representada por una función inicial de masa integrada galáctica (IGIMF, por sus siglas en inglés), propuesta por Kroupa \& Weidner (2003), cuya teoría fue desarrollada en la última década (ver Kroupa et al., 2013, para un artículo de revisión). Este formalismo establece que la formación estelar se lleva a cabo exclusivamente en cúmulos estelares (Lada \& Lada, 2003). De esta manera, las poblaciones estelares de cada galaxia están compuestas por estrellas en cúmulos estelares sobrevivientes y disueltos. Dentro de cada cúmulo estelar, las estrellas se forman con la IMF estelar dada por la ec. 3.12. Los cúmulos estelares inmersos en la galaxia también siguen una distribución inicial de masas de la forma $\xi_{\mathrm{ecl}}\left(M_{\mathrm{ecl}}\right) d M_{\mathrm{ecl}} \propto M_{\mathrm{ecl}}^{-\beta} d M_{\mathrm{ecl}}$, donde $M_{\mathrm{ecl}}$ es la masa del cúmulo inmerso. Además, la estrella más masiva en un cúmulo, $m_{\max }$, está relacionada de una manera no-trivial con la masa del cúmulo(Weidner et al., 2013); la masa de la estrella más masiva es mayor en cúmulos más masivos. Inclusive, las observaciones indican que mayores tasas de formación estelar (SFR, por sus siglas en inglés) llevan a la formación de cúmulos más brillantes (Larsen, 2002). Weidner et al. (2004) encuentran que esta correlación empírica se puede transformar en una relación entre la SFR de la galaxia y la masa máxima de cúmulo inmerso de la forma:

$$
M_{\mathrm{ecl}}^{\max }(S F R)=8,5 \times 10^{4} S F R^{0,75} \mathrm{M}_{\odot}
$$

La IGIMF es la suma de todas las estrellas recién nacidas en todos los cúmulos estelares considerando los ingredientes arriba mencionados, esto es,

$$
\xi_{\mathrm{IGIMF}}(m, t)=\int_{M_{\mathrm{ecl}}^{\min }}^{M_{\mathrm{ecl}}^{\max (\operatorname{SFR}(\mathrm{t}))}} \xi\left(m \leq m_{\max \left(\mathrm{M}_{\mathrm{ecl}}\right)}\right) \xi_{\mathrm{ecl}}\left(M_{\mathrm{ecl}}\right) d M_{\mathrm{ecl}},
$$

donde $\xi\left(m \leq m_{\max \left(\mathrm{M}_{\mathrm{ecl}}\right)}\right)$ es la IMF estelar dada por la ec. 3.12, y $M_{\mathrm{ecl}}^{\mathrm{min}}$ define la masa mínima de cúmulo que puede ser formada en una galaxia, la cual es un parámetro libre del modelo de IGIMF. Ahora, para esta formulación de la IGIMF, se supone que 
la formación estelar en todos los cúmulos estelares a toda época es producida con una IMF canónica. Sin embargo, las condiciones de formación estelar violenta pueden conducir a efectos de amontonamiento en cúmulos estelares masivos (Elmegreen, 2004; Shadmehri, 2004), y la formación de estrellas masivas puede ser favorecida bajo tales condiciones, dado que el límite de baja masa de formación estelar sería más alto. Siguiendo el trabajo preliminar de Marks et al. (2012), Weidner et al. (2011, WKP11 de aquí en más) consideran que, para cúmulos inmersos con $M_{\mathrm{ecl}}<2 \times 10^{5} \mathrm{M}_{\odot}$, la pendiente de la IMF canónica (eq. 3.12) para estrellas más masivas que $1,3 \mathrm{M}_{\odot}$ es $\alpha_{3}=2,35$. Para masas de cúmulo mayores, la dependencia de la pendiente de la IMF estelar para estrellas en este intervalo de masas está dada por

$$
\alpha_{3}\left(M_{\mathrm{ecl}}\right)=-1,67 \times \log _{10}\left(\frac{M_{\mathrm{ecl}}}{10^{6} \mathrm{M}_{\odot}}\right)+1,05 .
$$

El efecto de esta suposición en la IGIMF es analizado en WKP11. El extremo de altas masas de la IGIMF es, en general, más empinado que la IMF estelar, ya que la formación de estrellas de baja masa resulta favorecida. Sin embargo, si uno incluye el efecto de amontonamiento en cúmulos masivos, como hemos visto, la pendiente de la IMF canónica para estrellas masivas se puede volver más top-heavy (es decir, ms plana a altas masas) que la IMF de Salpeter para estos cúmulos. Cuando ocurre un evento tipo brote estelar con alta SFR en una galaxia, la formación de cúmulos masivos se ve favorecida y la formación de estrellas masivas es fuertemente aumentada, de manera que la IMF se puede volver también más top-heavy (con más estrellas a masas estelares altas, es decir, más plana) que Salpeter para estrellas de alta masa. Esta formulación ampliada es llamada: top heavy IGIMF (TH-IGIMF; WKP11). La pendiente de esta TH-IGIMF por sobre $1,3 \mathrm{M}_{\odot}$ puede entonces ser calculada por ajustes de mínimos cuadrados. Así, la TH-IGIMF puede ser traducida a una ley de potencias de una forma similar a la IMF estelar canónica usada para derivar la IGIMF, pero con un exponente $\alpha_{\mathrm{TH}}$ para estrellas de alta masa que depende de la SFR, esto es,

$$
\xi_{\mathrm{TH}}(m)=k \begin{cases}\left(\frac{m}{m_{\mathrm{H}}}\right)^{-\alpha_{1}} & , m_{\mathrm{H}} \leq \frac{m}{M_{\odot}}<m_{0}, \\ \left(\frac{m_{0}}{m_{\mathrm{H}}}\right)^{-\alpha_{1}}\left(\frac{m}{m_{0}}\right)^{-\alpha_{2}} & , m_{0} \leq \frac{m}{M_{\odot}}<m_{1}, \\ \left(\frac{m_{0}}{m_{\mathrm{H}}}\right)^{-\alpha_{1}}\left(\frac{m_{1}}{m_{0}}\right)^{-\alpha_{2}}\left(\frac{m}{m_{1}}\right)^{-\alpha_{\mathrm{TH}}} & , m_{1} \leq \frac{m}{M_{\odot}} \leq m_{\max } .\end{cases}
$$


donde $m_{\mathrm{H}}=0,1, m_{0}=0,5$ y $m_{1}=1,3$.

Luego de haber introducido los aspectos básicos de la teoría de la TH-IGIMF, describiremos la manera en la cual es implementada en SAG. Para cada evento de formación estelar, producido ya sea en modo quiescent o en brote estelar, le asignamos la TH-IGIMF dada por la ec. 3.16, de acuerdo a la correspondiente SFR. Es importante notar que, en nuestro modelo, consideramos $m_{\mathrm{H}}=0,1 M_{\odot}$ para el límite inferior en la TH-IGIMF, que es el límite adoptado en nuestra implementación química. Consideramos funciones de masa de cúmulos inmersos caracterizadas por valores distintos del exponente $\beta$, y diferentes masas mínimas de cúmulo inmerso $M_{\mathrm{ecl}}^{\mathrm{min}}$. Exploramos aquellos casos caracterizados por (i) $\beta=2 \mathrm{y}_{\text {ecl }}^{\min }=5 \mathrm{M}_{\odot}$, (ii) $\beta=2,1 \mathrm{y} M_{\mathrm{ecl}}^{\min }=5 \mathrm{M}_{\odot}, \mathrm{y}$ (iii) $\beta=2$ y $M_{\mathrm{ecl}}^{\mathrm{min}}=100 \mathrm{M}_{\odot}$. El modelo considera pequeñas variaciones de $\log (S F R)$ de manera que la pendiente de la TH-IGIMF esté bineada en pasos de 0,05. Es importante mencionar que algunas asociaciones estelares con masas tan bajas como $5 \mathrm{M}_{\odot}$ son encontradas en la galaxia (e.g. Taurus-Auriga star-forming regions, Kirk \& Myers, 2011), favoreciendo así el valor más bajo de $M_{\mathrm{ecl}}^{\mathrm{min}}$ considerado aquí. Por eso, al menos en condiciones de formación estelar quiescent, la $M_{\mathrm{ecl}}^{\mathrm{min}}$ está apoyada observacionalmente. No obstante, esta restricción observacional podría no ser válida en condiciones más extremas de formación estelar.

La Fig. 3.1 muestra la pendiente $\alpha_{\mathrm{TH}}$ como función de la SFR para diferentes valores de $M_{\mathrm{ecl}}^{\mathrm{min}}$ y $\beta$. Queda claro de este gráfico que la pendiente $\alpha_{\mathrm{TH}}$ de la TH-IGIMF es mayor que la pendiente de la IMF de Salpeter (caracterizada por un valor fijo de $\alpha=2,35$ para toda SFR) en regímenes de menor formación estelar. Los valores de SFR a los cuales la TH-IGIMF se vuelven más top heavy que Salpeter dependen del valor de $\beta$ y $M_{\mathrm{ecl}}^{\mathrm{min}}$, siendo del orden de 1,10 y $1000 \mathrm{M}_{\odot} \mathrm{yr}^{-1}$ para $\beta=2$ y $M_{\mathrm{ecl}}^{\min }=100 \mathrm{M}_{\odot}, \beta=2 \mathrm{y}$ $M_{\mathrm{ecl}}^{\min }=5 \mathrm{M}_{\odot}, \mathrm{y} \beta=2,1 \mathrm{y} M_{\mathrm{ecl}}^{\min }=5 \mathrm{M}_{\odot}$, respectivamente.

Durante períodos de alta actividad de formación estelar, la formación de estrellas masivas es favorecida. Para SFRs más altas, el número de estrellas de alta masa en una TH-IGIMF se vuelve mayor que en una IMF de Salpeter, con la correspondiente reducción del número de estrellas de baja masa requeridas por la normalización de la IMF. Este aspecto es crucial para entender la construcción de la relación entre el cociente de abundancias $[\alpha / \mathrm{Fe}]$ y la masa estelar de galaxias elípticas, como veremos en el Capítulo 4. 


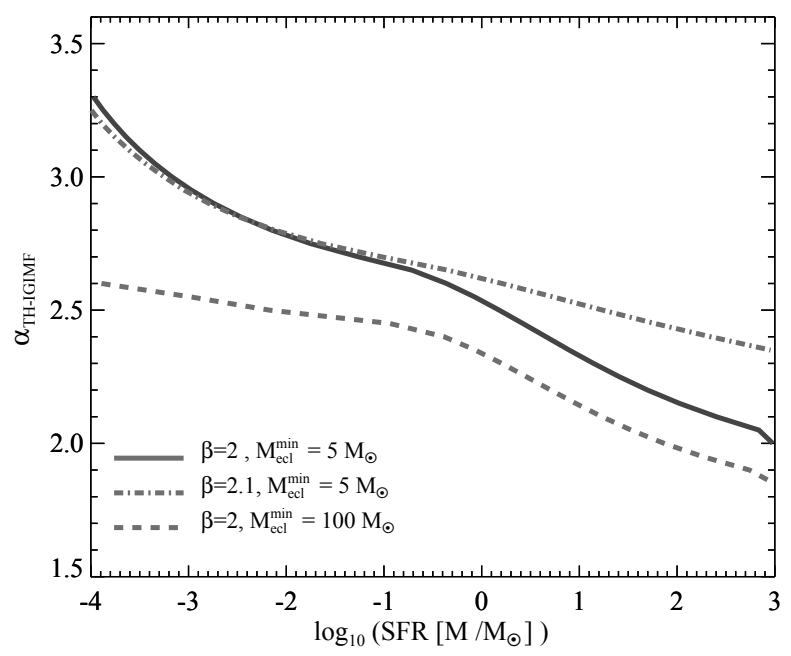

Figura 3.1: Dependencia de la pendiente de la TH-IGIMF $\left(\alpha_{\mathrm{TH}}\right)$ con la SFR para diferentes masas mínimas de ćumulo inmerso y pendiente de la función de masa de cúmulos inmersos: (i) $\beta=2, M_{\mathrm{ecl}}^{\mathrm{min}}=5 \mathrm{M}_{\odot}$ (línea sólida), (ii) $\beta=2,1, M_{\mathrm{ecl}}^{\mathrm{min}}=5 \mathrm{M}_{\odot}$ (línea de rayas y puntos), and (iii) $\beta=2, M_{\mathrm{ecl}}^{\min }=100 \mathrm{M}_{\odot}$ (línea a rayas).

\section{Brotes extendidos en SAG}

En versiones previas del modelo, la formación de estrellas en el bulbo ocurre a partir de brotes estelares que consumen el gas frío disponible en un paso único ${ }^{2}$, es decir, en forma instantánea. Ahora consideramos que los brotes estelares están caracterizados por una escala de tiempo dada, durante la cual el gas frío llevado al centro galáctico es consumido gradualmente en varios pasos. Así, este gas frío tiene la posibilidad de ser progresivamente contaminado por las estrellas formadas en el bulbo. De esta manera, el cociente de abundancias de $[\alpha / \mathrm{Fe}]$ de generaciones sucesivas de estrellas en el bulbo no queda sólo determinado por las abundancias del gas frío del disco al momento preciso en el cual un brote estelar es desencadenado, sino que también es modificado por la contribución relativa de diferentes tipos de SNs que logran contaminar el medio interestelar o no, dependiendo de la relación entre la escala de tiempo de la duración del brote y el tiempo de vida de los progenitores de SNs. El gas frío que eventualmente será convertido en estrellas del bulbo es denominado como gas frío del bulbo, con el objetivo

\footnotetext{
${ }^{2}$ Las ecuaciones diferenciales en SAG son integradas en pasos de tiempo de igual tamaño usados para subdividir los intervalos entre las salidas de las simulaciones de materia oscura.
} 
de diferenciarlo del gas frío del disco. La cantidad de gas frío del bulbo disponible nos permite realizar una primera estimación de la masa en estrellas que va a ser formada en el bulbo. Entonces, calculamos la escala de tiempo en la cual éstas se formarían, que es determinada como el tiempo dinámico considerado en la formación estelar quiescent como primera aproximación. Esto es, aquel definido como el cociente entre el radio de escala del perfil exponencial que caracteriza al disco galáctico y su velocidad circular. Esta elección parece razonable, ya que un brote normalmente ocurre conducido a través de una barra inmersa en el disco galáctico. Así, del cociente de la masa de gas frío del bulbo y la escala de tiempo estimada para la duración del brote estelar, conocemos la masa en estrellas que será formada en cada paso de tiempo. Por simplicidad, la formación estelar a partir de brotes extendidos ocurre a una tasa constante. Otros trabajos en la literatura que han tenido en cuenta las escalas de tiempo de los brotes, consideran que la tasa de formación estelar durante un brote decae exponencialmente con el tiempo luego de que el brote es disparado (Granato et al., 2000; Lacey et al., 2008). Justificamos nuestra suposición considerando que la barra formada durante la inestabilidad, accionada tanto por una fusión galáctica o una inestabilidad de disco, favorece la alimentación gradual del reservorio de gas frío del bulbo por el gas frío del disco que está siendo conducido hacia el centro galáctico (e.g. Kormendy \& Kennicutt, 2004), manteniendo constantes las condiciones que dan lugar a la formación estelar. Notar que la presencia de una barra durante el proceso de formación del bulbo está sólamente implícita en la suposición de la tasa de formación estelar constante durante los brotes de formación. Modelar la formación de las barras no es una tarea fácil (see Kormendy \& Kennicutt, 2004; Athanassoula, 2013), y está más allá del alcance de esta tesis. En el capítulo 4 se realiza un anális detallado del efecto de la implementación de los brotes extendidos en el enriquecimiento químico de las galaxias elípticas.

\section{Propiedades Espectro-Fotométricas con la TH-IGIMF}

El modelo semianalítico SAG calcula las propiedades físicas de las galaxias. Las salidas del modelo incluyen las masas estelares de las galaxias y sus componentes la masa de gas frío y caliente, y las respectivas masas en elementos químicos, por nombrar algunos ejemplos. Con el objetivo de poder comparar las salidas del modelo con cantidades observadas, el modelo incluye el cálculo de luminosidad y magnitudes inte- 
gradas de las galaxias en diferentes bandas fotométricas. Las magnitudes son calculadas a partir de modelos de síntesis de poblaciones que dependen de la metalicidad inicial del gas del cual se forman las estrellas y de la IMF. Usando estos modelos se puede conocer cómo contribuye una población estelar (normalizada por su masa) a la distribución espectral de energía. Cada vez que se forman estrellas en el modelo, dada una IMF y una tabla adecuada de tiempos de vida estelar, se calcula la proporción emitida en cada banda desde el instante de formación hasta el final de la vida de esas estrellas, y luego se integra sobre todas las poblaciones formadas. SAG calcula de esta manera magnitudes absolutas en las bandas U, B, V, K, R, I del sistema de Johnson-Cousins (Bessell, 1990) y las bandas u, g, r, i y z del SDSS (Fukugita et al., 1996).

Los modelos de síntesis de poblaciones utilizados corresponden a los modelos de Bruzual \& Charlot (2003) y Bruzual (2007). Como estos modelos tienen una dependencia explícita con la IMF adoptada, fueron generados en conjunto con Gustavo Bruzual, para todas las pendientes posibles de la TH-IGIMF de interés en este trabajo. Los modelos sin publicar de 2007 incluyen la receta final de Marigo \& Girardi (2007) y Marigo et al. (2008) para la evolución de estrellas de pulsaciones térmicas de la rama asintótica de las gigantes (TP-AGB por sus siglas en inglés). Esto produce colores en el IR-cercano significativamente más rojos, menores edades y masas más bajas para poblaciones estelares jóvenes y de edades intermedias, que sus contrapartes en 2003; ver Bruzual (2007) para más detalles.

El panorama no está completo si no se incluye la atenuación por polvo. Los granos de polvo presentes en el medio intestelar de las galaxias absorben la radiación estelar energética y vuelven a emitirla en frecuencias menos energéticas, enrojeciendo los colores de las galaxias. El modelo SAG utiliza el modelo motivado observacionalmente y presentado por Kauffmann et al. (1999). Ellos hacen uso de una correlación observada por Wang \& Heckman (1996), quienes encontraron una vinculación entre la luminosidad de la galaxia y la profundida óptica del polvo en una galaxia de disco vista de frente. La expresión en banda B de la correlación toma la forma

$$
\tau_{\mathrm{B}}=(0,8 \pm 0,3)\left(\frac{L_{\mathrm{B}}}{L_{\mathrm{B} \star}}\right),
$$

donde $L_{\mathrm{B}}$ es la luminosidad intrínseca y $L_{\mathrm{B} \star}$ es la luminosidad observada de una galaxia de magnitud $M_{\mathrm{B}}=-19,6+5 \log (\mathrm{h})$. Luego se utiliza el cociente $\tau_{\lambda} / \tau_{\mathrm{B}}$ para calcular la 
extinción en otras bandas fotométricas, aplicando una curva de extinción de la galaxia dada por Cardelli et al. (1989). Sumado a esto se supone una dependencia de la atenuación por polvo con el ángulo de la visual, $\theta$. El modelo SAG asigna de manera aleatoria a cada galaxia el ángulo de inclinación. La extinción efectiva de la fuente expresada en magnitudes resulta

$$
A_{\lambda}=-2,5 \log \left(\frac{1-\exp \left(-\tau_{\lambda} \sec \theta\right)}{\tau_{\lambda} \sec \theta}\right)
$$

\section{Calibración del modelo}

El modelado semianalítico involucra el sintonizado de los parámetros libres a través de su calibración frente a un conjunto de observaciones. Para este propósito, usamos la técnica de optimización de enjambres de partículas (Kennedy \& Eberhart 1995) aplicada a nuestro modelo semianalítico SAG. La descripción detallada del método está dada en Ruiz et al. (2014). Este procedimiento involucra la variación de un conjunto seleccionado de parámetros libres del modelo dentro de un dado intervalo de valores. Con este método novedoso, podemos explorar un gran espacio de parámetros y encontrar el mejor máximo global de la superficie de probabilidades. Los parámetros libres que son sintonizados están asociados al modelado de los principales procesos físicos incluidos en el código, desarrollados en las diferentes secciones de este capítulo. Estos son la eficiencia de formación estelar $(\alpha)$, la eficiencia de retroalimentación de $\mathrm{SN}$ asociada a la formación estelar que ocurre en el disco $(\varepsilon)$ y en el bulbo $\left(\varepsilon_{\text {bulge }}\right)$, los parámetros relacionados al crecimiento del agujero negro central supermasivo $\left(f_{\mathrm{BH}}\right)$ y la eficiencia de retroalimentación de AGN $\left(\kappa_{\mathrm{AGN}}\right)$, el parámetro involucrado en el accionamiento de las inestabilidades de disco $\left(f_{\text {pert }}\right)$, y la fracción de estrellas binarias $\left(A_{\text {bin }}\right)$.

En el capítulo siguiente describimos los resultados de la aplicación de este método de calibración a distintas variantes del modelo SAG, caracterizadas por distintas IMFs, que dan lugar a distintas versiones del modelo que serán usados luego para llevar a cabo el trabajo de investigación propuesto. 


\title{
Capítulo 4
}

\section{Downsizing quemo-arqueológico en un universo jerárquico: impacto de una función de masa inicial galáctica \\ integrada}

\begin{abstract}
'I wished to find out whether a similar bright line could be seen in the spectrum of sunlight as in the spectrum of lamplight, and I found, with the telescope, instead of this, an almost countless number of strong and feeble vertical lines which, however, were darker than the other parts of the spectrum, some appearing to be almost perfectly black.'
\end{abstract}

-Joseph Von Fraunhofer, Gesammelte Schriften (1888).

\section{Introducción}

Las propiedades químicas impresas en las poblaciones estelares son características esenciales para comprender la formación y evolución de las galaxias. La abundancia de elementos $\alpha$ relativa al hierro ha sido reconocida desde hace tiempo como un indicador 
de las escalas de tiempo de formación de poblaciones estelares. Es un hecho bien conocido que los elementos $\alpha$ son producidos por el proceso de captura de partículas $\alpha$ exclusivamente en supernovas con colapso de núcleo (SN II) cuyos progenitores tienen tiempos de vida de decenas de millones de años (Myr, por sus siglas en inglés), mientras que el hierro y los elementos cercanos al pico del hierro son principalmente yields de supornovas tipo Ia (SN Ia), cuyos progenitores son de vida larga (0,5 - 3 Gyr, Greggio, 2005). Dada una población estelar compuesta con un exceso de elementos $\alpha$ con respecto al hierro se supone, por lo tanto, que se forma en un período corto de tiempo ( $\lesssim 1 \mathrm{Gyr}$ ) (Matteucci, 1994), dado que la mayoría de las estrellas en el sistema se forma antes de que el medio interestelar (ISM, por sus siglas en inglés) sea contaminado con los yields de SN Ia. Los primeros trabajos de Tinsley $(1976,1979)$ ya consideraron a este cociente de abundancias para explicar las escalas de tiempo de formación de las estrellas del vecindario solar y el halo estelar de la Vía Láctea. Este argumento también ha sido considerado para explicar la tendencia observada entre las galaxias elípticas, en la cual las elípticas más masivas (con mayor dispersión de velocidad) tienen sistemáticamente mayores cocientes entre elementos $\alpha$ y hierro (e.g. Worthey et al., 1992). Faber (1973) observaron por primera vez que algunos rasgos de absorción en los espectros, relacionados a la abundancia de magnesio, crecen monótonamente con el incremento de la luminosidad. Desde aquel entonces, una gran cantidad de trabajo se ha realizado midiendo las abundancias de elementos $\alpha$ para estudiar esta tendencia en todo tipo ambientes (e.g. Worthey et al., 1992; Trager et al., 2000; Kuntschner, 2000; Bernardi et al., 2006; Sánchez-Blázquez et al., 2006; Spolaor et al., 2010, T10). En el contexto del paradigma $\Lambda \mathrm{CDM}$, la tendencia observada de $[\alpha / \mathrm{Fe}]$ como función de la masa podría ser considerada problemática. En este marco, el crecimiento de la estructura es jerárquico. Los halos de DM se forman vía colapso gravitatorio sin disipación (White \& Rees, 1978b) y crecen de menor a mayor tamaño, de manera que los halos de mayor tamaño tardan más tiempo en acretar su masa. Si consideramos que las galaxias se forman en los centros de los halos de DM, a priori no se espera que las galaxias más masivas tengan abundancias de elementos $\alpha$ relativas al hierro más altas. Sin embargo, la existencia de procesos bariónicos como el enfriamiento del gas, la formación estelar, el feedback de SNs y de los núcleos galácticos activos pueden apartar la formación de galaxias real del ensamblado del halo de MO que la contiene. Este comportamiento "antijerárquico", usualmente llamado downsizing, se manifiesta de diferentes maneras en las galaxias. La 
masa característica de las galaxias con niveles de formación estelar alta, se incrementa con el redshift (e.g. Cowie \& Barger, 2008), la evolución de las funciones de masa estelar parecen indicar que las galaxias menos masivas ensamblan su masa a tiempos más tardíos (e.g. Pérez-González et al., 2008), las galaxias más masivas tienen poblaciones estelares más viejas que las menos masivas (e.g. Gallazzi et al., 2005). Fontanot et al. (2009) clasifica las diferentes manifestaciones observacionales del downsizing sugiriendo la posibilidad de que no todos estos fenómenos estén relacionados al mismo proceso físico subyacente. En particular, ellos acuñaron el término chemo-archaeological downsizing para referirse a la tendencia de cocientes de abundancia en relación a la masa mostrada por las galaxias elípticas.

La tensión señalada entre las observaciones y el paradigma jerárquico ha sido abordada por diferentes grupos usando modelos analíticos y semianalíticos de formación de galaxias. Thomas (1999) fue el primero en modelar el enriquecimiento químico de galaxias elípticas dentro del marco de la formación galáctica jerárquica, pero considerando un modelo de caja cerrada para cada galaxia e ignorando la historia de fusiones de las galaxias al usar historias de formación estelar precalculadas. El autor falla en reproducir la tendencia observada de $[\alpha / \mathrm{Fe}]$ en función de la dispersión de velocidades. Nagashima et al. (2005) estudian el enriquecimiento en metales de galaxias elípticas usando un modelo semianalítico con árboles de fusiones. Ellos encuentran que una función inicial de masa más top-heavy en brotes de formación estelar es necesaria para reproducir el cociente de abundancia $[\alpha / \mathrm{Fe}]$ en elípticas con luminosidades $L_{\star}\left(L_{\star}\right.$ es la luminosidad característica en una función de Schechter en una banda dada), pero ninguno de sus modelos reproduce la tendencia observada de este cociente con la dispersión de velocidades. Pipino et al. (2009) se concentran en la relación $[\alpha / \mathrm{Fe}]$-masa estelar en galaxias elípticas incluyendo el enriquecimiento de SNs tipo Ia y II, y de estrellas de masa baja e intermedia, como también los tiempos de retardo de explosión de SN Ia, en el modelo semianalítico GalICs. Ellos encuentran un mejor acuerdo con la relación $[\alpha / \mathrm{Fe}]-\sigma$ observada cuando incluyen el feedback de AGN en sus modelos, aunque aún marginal. Calura \& Menci (2009) implementan un modelo químico sobre historias de formación estelar extraídas de un modelo semianalítico y evalúan el impacto del feedback de AGN y de una IMF variable ad-hoc, que se vuelve top-heavy en brotes estelares y encuentran que ambos cambios son necesarios para obtener una pendiente en la relación similar a la observada. Este fue el primer intento de incluir una función inicial de masa variable en 
un modelo semianalítico para reproducir con éxito la tendencia observada en la relación $[\alpha / \mathrm{Fe}]$-masa en galaxias elípticas. Una suposición similar sobre la IMF ha sido considerada por Baugh et al. (2005) para explicar el número de cuentas de galaxias submilimétricas a alto redshift. Más tarde, Calura \& Menci (2011) mencionan la importancia del harassment de galaxias satélites a alto redshift como un disparador de formación estelar en los progenitores de galaxias masivas que forman estrellas enriquecidas en elementos $\alpha$, contribuyendo a construir la relación. Arrigoni et al. (2010) también analizan la relación $[\alpha / \mathrm{Fe}]-m a s a$ usando el modelo semianalítico de Somerville et al. (2008), que incluye feedback de AGN, en el cual implementan un modelo de enriquecimiento químico que involucra yields de SN Ia y II, y los correspondientes tiempos de retardo de las explosiones de SNIa. Ellos encuentran un buen acuerdo con las observaciones combinando una pendiente plana para la IMF y una menor fracción de sistemas binarios que terminan sus vidas como SN Ia. Todos los resultados obtenidos de modelos semianalíticos de formación y evolución de galaxias sugieren que el argumento de escalas de tiempo de formación, que compara las escalas de tiempo de formación estelar y la eyección de metales por las SNs, podría ser insuficiente para explicar la construcción de la relación de $[\alpha / \mathrm{Fe}]$-masa estelar en galaxias de tipo temprano, apoyando la importancia del rol de la IMF. Están siendo publicados numerosos trabajos que muestran la necesidad de explorar funciones iniciales de masa alternativas y variables.

La IMF tiene consecuencias directas en la evolución de las galaxias. Ésta determina el cociente de estrellas masivas sobre estrellas de masa baja e intermedia, imprimiendo los cocientes de abundancias químicas de las poblaciones estelares en galaxias (Lucatello et al., 2005; Köppen et al., 2007), y estableciendo la cantidad de masa recalentada depositada en el ISM por las explociones de SNs. También determina el cociente de masa-luminosidad de las galaxias, dado que estrellas con diferente masa estelar inicial contribuyen con muy distintas cantidades de luz a lo largo de sus vidas. En la última década se ha desarrollado un formalismo para una IMF estelar para las galaxias como un todo (ver Kroupa et al., 2013, o la subsección 8.2 de esta tesis), donde la tasa de formación estelar (SFR) instantánea de las galaxias determina la forma final de esta IMF integrada. Recchi et al. (2009) evalúan la influencia de la IMF integrada galáctica (IGIMF, Kroupa \& Weidner, 2003) sobre la relación $[\alpha / F e]-\sigma$ implementando esta nueva IMF en un modelo analítico de enriquecimiento químico de galaxias. Ellos encuentran que el efecto de downsizing (menor duración de la formación estelar en ga- 
laxias más grandes) debe estar presente para reproducir la tendencia observada, aunque debe ser más debil que aquella inferida por Thomas et al. (2005) en base a la relación $[\alpha / \mathrm{Fe}]-\sigma$ observacional. En su trabajo se adoptaron varias suposiciones simplificadoras. Además, no estudian el efecto de una versión Top-heavy de la IGIMF (TH-IGIMF) (Weidner et al., 2011, 2013).

El objetivo de este capítulo es abordar la evolución química de las galaxias elípticas en el marco del paradigma $\Lambda \mathrm{CDM}$, abordando el problema del enriquecimiento de elementos $\alpha$ en las mismas. Evaluamos el impacto de la nueva teoría de TH-IGIMF, nunca considerada hasta ahora para este tema en particular. También estudiamos la influencia de la TH-IGIMF en la función luminosidad y los cocientes masa-luminosidad (M/L) de las galaxias elípticas, analizando la inclinación del plano fundamental (M/L vs $M)$. Para este propósito, usamos el modelo semianalítico SAG descripto en el Capítulo 3 que reúne los ingredientes más relevantes de la evolución química junto con la física involucrada en la formación y evolución de las galaxias (Cora 2006; Lagos, Cora \& Padilla 2008a; Tecce et al. 2010) y en el cual se implementaron los cambios referentes a los brotes estelares extendidos y a la TH-IGIMF (Gargiulo et al., 2014).

Este capítulo se organiza de la siguiente manera. En la Sección 2 detallamos la forma en que se calculan las cantidades involucradas en la relacion $[\alpha / \mathrm{Fe}]-$ masa estelar a partir de los datos dados por el modelo. En las Sección 3 mostramos los resultados de la calibración del modelo con la IMF universal de Salpeter considerando dos conjuntos de restricciones observacionales, permitiéndonos definir el conjunto a usar para calibrar el modelo con diferentes variantes de la TH-IGIMF, es decir, con diferentes parámetros de la teoría. En la Sección 4, analizamos el impacto de la TH-IGIMF en el desarrollo de la relación $[\alpha / \mathrm{Fe}]$-masa estelar. En la Sección 5 investigamos el efecto de los brotes extendidos y tiempos de retardo de SNs. Luego, discutimos la influencia de las escalas de tiempo involucradas en el modelado de la formación de galaxias elípticas en la Sección 6. En la Sección 7, mencionamos investigaciones previas en el campo, poniendo en contexto nuestros resultados. Presentamos las conclusiones en el Capítulo 6 de la tesis. 


\section{Construcción de la relación $[\alpha / F e]-m a s a$ estelar}

El objetivo de este capítulo es identificar los aspectos principales involucrados en la construcción de la relación $[\alpha / \mathrm{Fe}]$-masa estelar de galaxias elípticas. Nuestro modelo semianalítico provee la información necesaria para construir esta relación. Clasificamos una galaxia como de tipo temprano si el bulbo galáctico representa más del 80 por ciento de la masa bariónica total de la galaxia, esto es, adoptamos $(\mathrm{B} / \mathrm{T})_{\text {thresh }}=0,8$. (ver subsección 5). Con este criterio, estimamos la fracción de galaxias de tipo temprano y tardío. Las galaxias sin bulbo son consideradas como irregulares.

Una vez seleccionada la población de galaxias de tipo temprano en el modelo, estimamos el cociente de abundancias $[\alpha / \mathrm{Fe}]$ de su componente estelar. Siguiendo a Geisler et al. (2007), definimos el cociente de abundancias $[\alpha / \mathrm{Fe}]$ como

$$
[\alpha / \mathrm{Fe}]=\frac{1}{6} \sum_{i=1}^{6}[\alpha / \mathrm{Fe}]_{i}
$$

donde $[\alpha / \mathrm{Fe}]_{i}$ denota el logaritmo del cociente de abundancia respecto al hierro de cada uno de los seis elementos- $\alpha$ disponibles en nuestra implementación química. $\left({ }^{16} \mathrm{O}\right.$,

$\left.{ }^{20} \mathrm{Ne},{ }^{24} \mathrm{Mg},{ }^{28} \mathrm{Si},{ }^{32} \mathrm{~S},{ }^{40} \mathrm{Ca}\right)$. Buscando comparar con los datos observacionales considerados, referimos los cocientes de abundancia de galaxias en el modelo a las abundancias solares de Grevesse et al. (1996) como en T10 y Arrigoni et al. (2010).

\section{Calibraciones}

En la Sección 11 del Capítulo 3 introdujimos el método de calibración utilizado para sintonizar los parámetros libres del modelo semianalítico SAG. En esta sección presentamos los conjuntos de restricciones observacionales utilizados y los resultados de las calibraciones para los distintos modelos probados.

\subsection{Restricciones observacionales}

Evaluamos el comportamiento del modelo considerando dos conjuntos distintos de restricciones observacionales para calibrar los parámetros libres. El primer conjunto involucra la luminosidad a $z=0$ en la banda- $r$ (LF en banda-r), la relación entre la 
masa del agujero negro supermasivo y la masa del bulbo (relación BHB, por sus siglas en inglés) y la evolución con el redshift de las tasas de SNs Ia y SNs II. El segundo conjunto también incluye la relación $[\alpha / \mathrm{Fe}]$-masa estelar en galaxias elípticas. La LF es una restricción fundamental para modelos semianalíticos de formación de galaxias, ya que refleja la influencia de la formación estelar y los procesos de retroalimentación energética. El extremo poco brillante de la LF está asociado principalmente a la retroalimentación de SNs. Este aspecto es relevante para nuestro trabajo, porque las diferentes IMFs contribuyen con diferentes números de SNs II, siendo pocas (muchas) para IMFs con valores grandes (bajos) en sus pendientes, y los parámetros de eficiencia de retroalimentación, $\varepsilon$ y $\varepsilon_{\text {bulge }}$ deben estar debidamente sintonizados para producir resultados que no subestimen (sobrestimen) el efecto mencionado. Por otro lado, el quiebre en la LF de las galaxias y la caída en el extremo más brillante está determinado principalmente por la retroalimentación de AGN. Se cree que este proceso es responsable del apantallamiento de la formación estelar en galaxias masivas, contribuyendo al comportamiento de downsizing de las galaxias. La retroalimentación de AGN también es restringido a través de la relación $\mathrm{BHB}$, ya que el agujero negro central supermasivo es el responsable de este proceso. Restringiendo la masa en bulbos en galaxias a través de la relación BHB ayuda también a obtener una mezcla adecuada de morfologías en las galaxias.

La evolución de las tasas de SNs Ia y II son particularmente importantes en este estudio, dado que el número de diferentes tipos de SNs y sus correspondientes yields tienen un impacto directo en el cociente de abundancias $[\alpha / \mathrm{Fe}]$ de las estrellas. La evolución de la tasa de SNs II no sólo determina la cantidad de elementos $\alpha$ producidos a lo largo del tiempo de vida de las galaxias, sino que constituye un diagnóstico alternativo de la evolución de la SFR, como consecuencia de la corta vida de los progenitores de SNs II. Reproducir la SFR observada es una condición requerida para cualquiera de nuestros modelos, pero es particularmente relevante para aquéllos que involucran una TH-IGIMF por la dependencia que existe en su pendiente con la SFR. De hecho, la pendiente de la TH-IGIMF determina el número de SNs II y, consecuentemente, la retroalimentación que contribuye a regular el proceso de formación estelar. Uno de los parámetros de calibración, $A_{\text {bin }}$, regula la cantidad de sistemas binarios que explotan como SNs Ia, y por lo tanto, la cantidad de hierro reciclado en el ISM que estará disponible durante la formación de futuras generaciones de estrellas. Así, es crucial restringir su valor para evitar realizar conclusiones erróneas con respecto al cociente de abundancias $[\alpha / \mathrm{Fe}]$ en 
estrellas. Vamos a retomar el análisis de las escalas de tiempo de las SNs y el impacto del parámetro $A_{\text {bin }}$ en las escalas de tiempo de retardo (de explosión de SNs Ia) en la Sección 5.2.

Los datos observacionales que usamos para ambos conjuntos de restricciones son la LF en la banda-r de Blanton et al. (2005), la relación BHB dada por Häring \& Rix (2004) y Sani et al. (2010), y la compilación de tasas de SNs Ia y SNs II dada por Melinder et al. (2012). En el segundo conjunto de restricciones también consideramos el cociente de abundancias $[\alpha / \mathrm{Fe}]$ en función de la masa presentado en Thomas et al. (2010, T10 de aquí en más). Ellos analizan una submuestra obtenida de la liberación de datos 4 del Sloan Digital Sky Survey (SDSS) (Adelman2006, 2006, DR4), seleccionada morfológicamente por inspección visual. La submuestra es seleccionada en el intervalo de redshift $0,05<z<0,06$ para evitar bias en la velocidad de dispersiones. Obtienen los cocientes de abundancias de $[\alpha / \mathrm{Fe}]$ para cada galaxia en su muestra por medio de índices espectrales de Lick observados y modelos de sínstesis de poblaciones estelares que tienen en cuenta efectos de cocientes de abundancias de elementos. Ellos también calculan la masa dinámica de cada galaxia de la dispersión de velocidades en la línea de la visual usando la relación de escala presentada en Cappellari et al. (2006). Esta masa dinámica es considerada una buena medida de la masa bariónica de las galaxias elípticas, de tal manera que podemos comparar la relación de $[\alpha / \mathrm{Fe}]$-masa estelar obtenida de nuestro modelo con la relación $[\alpha / \mathrm{Fe}]$-masa dinámica presentada por T10. Los errores promedio de los valores observados de $[\alpha / \mathrm{Fe}]$ son del orden de 0,06 dex. También consideramos el conjunto de datos observacionales de Arrigoni et al. (2010), el cual fue obtenido por Trager et al. (2000) y re-analizado con modelos de síntesis de población descriptos en Trager et al. (2008).

\subsection{Resultados de las calibraciones}

En esta sección presentamos dos calibraciones realizadas para el modelo con una IMF de Salpeter, utilizando los dos grupos de restricciones disponibles y verificamos el impacto de las mismas en la relación $[\alpha / \mathrm{Fe}]$-masa estelar. 
Cuadro 4.1: Valores de los parámetros libres del modelo semianalítico con una IMF de Salpeter obtenidos a partir de los dos grupos de restricciones observacionales considerados, dando lugar a dos variantes del mismo modelo: SAGS-c1 y SAGS-c2.

\begin{tabular}{ccc}
\hline \hline & Salpeter IMF & Salpeter IMF \\
\hline Param & SAGS-c1 & SAGS-c2 \\
\hline$\alpha_{\mathrm{SF}}$ & 0.1271 & 0.2328 \\
$\varepsilon$ & 0.2263 & 0.2541 \\
$\varepsilon_{\text {bulge }}$ & 0.0513 & 0.0778 \\
frac $_{\mathrm{BH}}$ & 0.0139 & 0.0141 \\
$k_{\mathrm{AGN}}$ & $2.32 \times 10^{-5}$ & $4.34 \times 10^{-4}$ \\
$A_{\text {bin }}$ & 0.0341 & 0.0383 \\
$f_{\text {pert }}$ & 44.62 & 47.63 \\
\hline \hline
\end{tabular}

\section{Calibraciones para la IMF de Salpeter}

Consideramos el modelo SAG con IMF de Salpeter y lo calibramos usando los dos conjuntos de restricciones observacionales introducidas en la sección anterior, dando lugar a dos variantes del mismo modelo, referidos como SAGS-c1 y SAGS-c2. La inclusión de la relación $[\alpha / \mathrm{Fe}]$-masa estelar como una restricción adicional en el segundo conjunto impone más restricciones a los parámetros libres y sus valores cambian respecto a aquéllos obtenidos del primer conjunto de restricciones. Los valores de los parámetros obtenidos para cada calibración son presentados en la Tabla 4.1.

Es interesante notar cómo la calibración del modelo impacta sobre propiedades de las galaxias que no fueron utilizadas para su calibración. Una cantidad especialmente importante para la realización de este trabajo es la relación entre la masa del bulbo y la total (cociente $\mathrm{B} / \mathrm{T}$ ) de las galaxias, ya que ésta determina la fracción de galaxias elípticas presentes en el modelo. Para determinar cómo las calibraciones impactan en las propiedades morfológicas de las galaxias del modelo, comparamos los resultados con los resultados de fracciones morfológicas observadas. La fracción de galaxias con diferentes tipos morfológicos del modelo SAGS-c1 y SAGS-c2 son presentados en la Figura 4.1. En ambos casos, están en concordancia con la fracción observada en el Universo local, para el cual usamos datos de Conselice (2006). La relación $[\alpha / \mathrm{Fe}]-m a s a$ estelar para la población de galaxias elípticas, utilizada para la calibración del modelo SAG-c2, se muestra en la Figura 4.2 para SAGS-c1 (panel superior) y SAGS-c2 (panel 


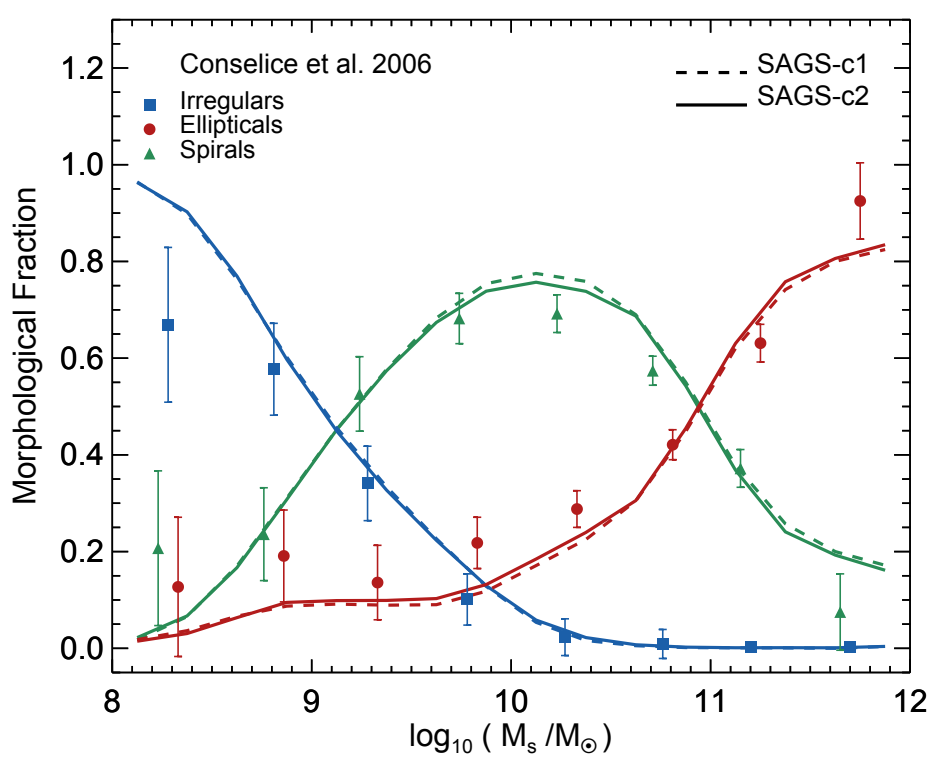

Figura 4.1: Fracción de galaxias de diferentes tipos morfológicos como función de la masa estelar para las dos variantes del modelo SAG con IMF de Salpeter resultantes de las calibraciones obtenidas a partir de dos grupos de restricciones observacionales: modelos SAGS-c1 (línea a rayas) y SAGS-c2 (línea sólida); las mismas son comparada con la mezcla morfológica observada en galaxias locales (Conselice, 2006). Las galaxias irregulares, espirales y elípticas en el modelo son representadas por diferentes colores, mientras las fracciones correspondientes inferidas de observaciones son representadas por triángulos, rombos y círculos con el mismo código de colores, como está indicado en las referencias del gráfico. Las galaxias elípticas en el modelo son aquéllas que satisfacen la condición $\mathrm{B} / \mathrm{T}>(\mathrm{B} / \mathrm{T})_{\text {thresh }} \equiv 0,8$.

inferior). Los resultados de los modelos son comparados con la relación $[\alpha / \mathrm{Fe}]$-masa dinámica de galaxias elípticas dada por T10, la cual está representada por contornos de color azul. El ajuste lineal a esta población de galaxias se muestra en la línea azul a rayas. La extrapolación de este ajuste a menores masas está en acuerdo con la tendencia mostrada por los datos de Arrigoni et al. (2010), representada por cuadrados azules.

Encontramos que, usando sólo la LF en la banda- $r$, la relación BHB y la evolución de las tasas de SNs como restricciones, el modelo no es capaz de reproducir la relación $[\alpha / \mathrm{Fe}]$-masa estelar observada. En la población de galaxias generada por SAGS-c1, las galaxias menos masivas alcanzan cocientes de abundancia de $[\alpha / \mathrm{Fe}]$ prácticamente iguales a las más masivas, dando lugar a un relación $[\alpha / \mathrm{Fe}]$-masa estelar con una ten- 


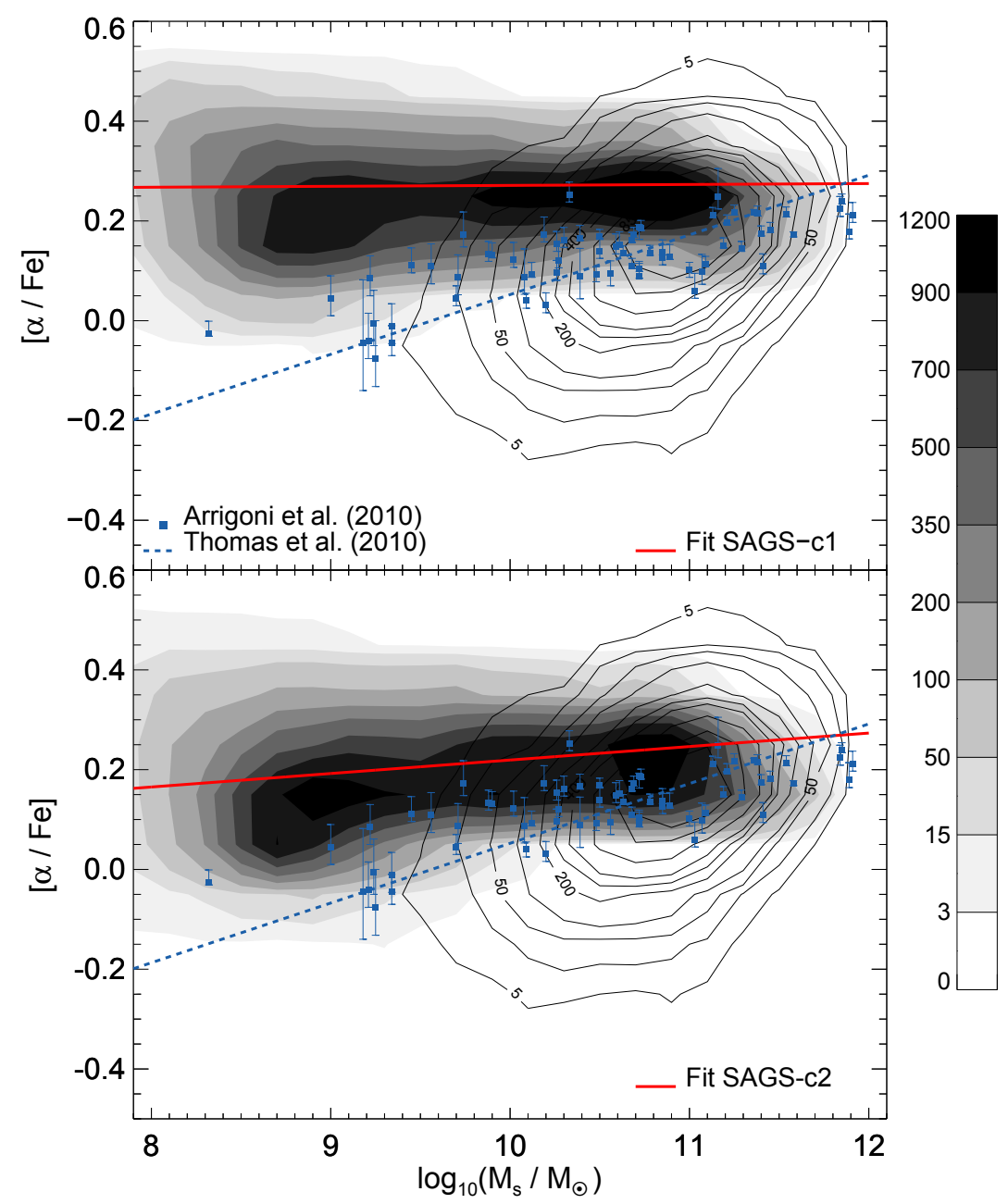

Figura 4.2: Relación $[\alpha / \mathrm{Fe}]$-masa estelar para galaxias elípticas en las versiones de SAG con una IMF de Salpeter resultantes de las calibraciones obtenidas a partir de dos grupos de restricciones observacionales: SAGS-c1 (panel superior) y SAGS-c2 (panel inferior). Las distribuciones de cocientes de abundancias son representadas por contornos de densidad en gris; las líneas sólidas rojas son los ajustes lineales correspondientes. Los contornos de líneas azules representan la relación trazada por la muestra de galaxias elípticas de T10; el ajuste a los cocientes de abundancia de $[\alpha / \mathrm{Fe}]$ para bines de masa estelar está marcado con una línea azul a rayas. Los símbolos azules corresponden a los datos de Arrigoni et al. (2010). 
dencia plana. Esto es reflejado en el ajuste lineal ${ }^{1}$ a la distribución de la población de galaxias tempranas completa, representado por la línea roja. Los contornos con código de grises denotan el número de galaxias elípticas del modelo.

Imponiendo la relación observada $[\alpha / \mathrm{Fe}]$-masa estelar como una restricción adicional permite restringir de manera correcta los valores de eficiencia de feedback de SN asociados a las estrellas del bulbo $\left(\varepsilon_{\text {bulge }}\right)$, y obtener una correlación positiva entre las abundancias $[\alpha / \mathrm{Fe}]$ y la masa estelar en el modelo SAGS-c2, en mejor acuerdo con la tendencia observada. El valor más grande de $\varepsilon_{\text {bulge }}$ obtenido del segundo conjunto de restricciones favorece la eyección de gas frío del bulbo en galaxias menos masivas. Este reservorio de gas puede ser reutilizado más tarde para la futura formación estelar en el bulbo con gas frío contaminado con una abundancia de hierro mayor producida por la contribución retardada de las SNs Ia.

Aunque la pendiente de la relación $[\alpha / \mathrm{Fe}]$-masa estelar para el modelo SAGS-c2 es positiva $(a=0,025)$, la misma sigue siendo casi plana; la pendiente del ajuste a los datos es de $a=0,1184$. Este resultado resalta la dificultad de los modelos semianalíticos (SAMs, por sus siglas en inglés) para reproducir adecuadamente la relación observada cuando se usa una IMF universal. Varios grupos de trabajo han intentado reproducir la pendiente de la relación $[\alpha / \mathrm{Fe}]$-masa estelar usando SAMs con una IMF universal durante la última década. Entre los más recientes, Arrigoni et al. (2010) encuentran que con una pendiente más top-heavy para la IMF, pueden lograr una pendiente positiva en la relación $[\alpha / \mathrm{Fe}]$-masa estelar, cercana a la observada. Más tarde, Calura \& Menci (2009) usan otro SAM y muestran que simplemente cambiando la pendiente de la IMF parece ser insuficiente para obtener la pendiente correcta en la relación observada. Ellos argumentan que la suposición de una IMF constante más plana que Salpeter sólo eleva el punto de cero de la relación. Reportamos aquí que cuando cambiamos la pendiente uniformemente (para todas las masas) de la IMF en SAG, también tenemos como resultado una variación en el punto de cero, sin un cambio apreciable en la pendiente, apoyando la última conclusión. Calura \& Menci (2011) incluyen los efectos de brotes estelares debido a interacciones de paso cercano a alto redshift estudiado por Menci et al. (2004) en su modelo semianalítico de formación de galaxias. Estas interacciones se supone que

\footnotetext{
${ }^{1}$ Usamos la rutina de IDL robust_linefit.pro (http://idlastro.gsfc.nasa.gov/ftp/pro/robust/robust_linefit.pro).
} 
activan brotes estelares por harrasment ${ }^{2}$ siguiendo el modelo desarrollado por Cavaliere $\&$ Vittorini (2000). Ellos encuentran que la pendiente de la relación [ $\alpha / \mathrm{Fe}]$-masa estelar se puede reproducir naturalmente cuando se agrega este efecto, aunque la pendiente que obtienen es más plana que la esperada por las observaciones (ver su Figura 1). El efecto de brotes estelares disparados por galaxias compañeras está presente en nuestro modelo SAG a través de nuestra implementación de inestabilidades de disco (ver Sección 5 del Capítulo 3). Forzando los parámetros para reproducir de la mejor manera la relación $[\alpha / \mathrm{Fe}]$-masa estelar con una IMF de Salpeter, esto es, considerando un grupo de restricciones observacionales que incluye la relación $[\alpha / \mathrm{Fe}]-$ masa estelar, obtenemos una pendiente similar a la del mejor modelo de Calura \& Menci (2011), aunque en ambos casos la pendiente es muy plana comparada con las observaciones. Aún restringiendo los parámetros con la relación misma, no es posible lograr una pendiente satisfactoria.

Este procedimiento ha mostrado la necesidad de incluir la relación $[\alpha / \mathrm{Fe}]-$ masa estelar como una restricción con el objetivo de limitar los valores adoptados por uno de los parámetros libres del modelo, De ahora en más, en el resto de este capítulo y los siguientes, las diferentes versiones de SAG resultantes de usar otras IMFs estarán calibradas usando el segundo conjunto de restricciones observacionales propuesto. Los resultados de estos modelos serán comparados con aquéllos del modelo SAGS-c2, al cual denominaremos simplemente SAGS.

\section{Calibraciones para la TH-IGIMF}

Probar nuevas recetas para representar procesos físicos usando un conjunto fijo de parámetros libres es una práctica común en el modelado semianalítico de propiedades de galaxias. Los parámetros libres son sintonizados para ajustar restricciones observacionales para una dada versión del modelo. Generalmente, los valores preferidos de estos parámetros son mantenidos para una versión modificada del modelo en el cual algunos aspectos físicos son cambiados con el objetivo de evaluar el impacto de las modificaciones introducidas en las propiedades de las galaxias resultantes. Esto es aceptable en la medida que la nueva versión arroje resultados en buen acuerdo con las restricciones observacionales elegidas. Sin embargo, dependiendo de las modificaciones introduci-

\footnotetext{
${ }^{2}$ Se denomina harrasment al fenómeno por el cual una galaxia ve intensificada su tasa de formación estelar debido a las perturbaciones dinámicas generadas por una galaxia satélite en un paso cercano a la galaxia central.
} 
Cuadro 4.2: Parámetros de calibración de los modelos SAGTH5B2, SAGTH5B21 y SAGTH100B2, los cuales fueron corridos con una TH-IGIMF caracterizada por distintas combinaciones de masa mínima de cúmulo estelar inmerso y pendiente de la función de masa de cúmulos estelares inmersos, $M_{\mathrm{ecl}}^{\min }=5 \mathrm{M}_{\odot}, \beta=2 ; M_{\mathrm{ecl}}^{\min }=5 \mathrm{M}_{\odot}, \beta=2,1 \mathrm{y}$ $M_{\mathrm{ecl}}^{\min }=100 \mathrm{M}_{\odot}, \beta=2$, respectivamente.

\begin{tabular}{cccc}
\hline \hline \multicolumn{3}{c}{ TH-IGIMF } \\
\hline Param & SAGTH5B2 & SAGTH5B21 & SAGTH100B2 \\
\hline$\alpha_{\mathrm{SF}}$ & 0.0312 & 0.0338 & 0.0619 \\
$\varepsilon$ & 0.3613 & 0.3532 & 0.2351 \\
$\varepsilon_{\text {bulge }}$ & 0.3163 & 0.413 & 0.0479 \\
frac $_{\mathrm{BH}}$ & 0.0516 & 0.0343 & 0.0765 \\
$k_{\mathrm{AGN}}$ & $7.92 \times 10^{-5}$ & $1.11 \times 10^{-4}$ & $9.09 \times 10^{-5}$ \\
$A_{\text {bin }}$ & 0.0386 & 0.04 & 0.0415 \\
$f_{\text {pert }}$ & 39.96 & 48.36 & 34.08 \\
\hline \hline
\end{tabular}

das, este procedimiento puede no llegar a resultar más válido. El estudio del impacto de diferentes IMFs en las propiedades químicas de galaxias elípticas llama al análisis de distintas versiones de SAG. La experiencia nos mostró que cuando la función inicial de masa es cambiada, las propiedades de las galaxias son modificadas fuertemente y es requerida una recalibración. En esta sección presentamos los valores de los parámetros libres resultantes de las calibraciones del modelo usando diferentes opciones de TH-IGIMF.

A partir de la implementación de la TH-IGIMF en la versión actualizada de SAG, tenemos la posibilidad de evaluar el impacto de considerar una variación de dos parámetros libres diferentes involucrados en la teoría de la IGIMF, que son la masa mínima de cúmulo inmerso y la pendiente de la función de masa de cúmulos estelares inmersos (ver Sección 8.2 del Capítulo 3). Así, tenemos tres diferentes nuevas versiones de SAG conrrespondientes a $M_{\mathrm{ecl}}^{\min }=5 \mathrm{M}_{\odot}, \beta=2 ; M_{\mathrm{ecl}}^{\min }=5 \mathrm{M}_{\odot}, \beta=2,1$ y $M_{\mathrm{ecl}}^{\min }=100 \mathrm{M}_{\odot}, \beta=2$, a los cuales nos referimos como modelos SAG1TH5B2, SAG1TH5B21 y SAG1TH100B2, respectivamente.

En todos los casos, cuando activamos la TH-IGIMF incluimos la relación $[\alpha / \mathrm{Fe}]-$ masa estelar como una restricción. La Tabla 4.2 muestra los valores de los parámetros adoptados en las nuevas calibraciones. 


\section{Relación $[\alpha / \mathrm{Fe}]-$ masa: Impacto de una TH-IGIMF}

El principal objetivo de este capítulo es evaluar el impacto de la TH-IGIMF variable en el desarrollo de la relación $[\alpha / \mathrm{Fe}]$-masa estelar en galaxias elípticas, motivados por el hecho de que con una IMF universal no somos capaces de reproducir la pendiente correcta de esta relación, aún cuando la relación misma es usada como restricción para la calibración del modelo.

\subsection{Impacto de una TH-IGIMF}

Nos enfocamos ahora en el impacto de la TH-IGIMF en la relación $[\alpha / \mathrm{Fe}]$-masa estelar para galaxias elípticas. La Fig. 4.3 muestra los resultados de los cocientes de abundancia $[\alpha / \mathrm{Fe}]$ de galaxias elípticas en función de su masa estelar para los modelos SAGTH5B2, SAGTH5B21 y SAGTH100B2 (paneles de la izquierda, medio y derecha, respectivamente); las correspondientes distribuciones de galaxias son mostradas en contornos de densidad grises. Las galaxias elípticas en el modelo son aquéllas que satisfacen la condición $\mathrm{B} / \mathrm{T}>(\mathrm{B} / \mathrm{T})_{\text {thresh }} \equiv 0,8$. Con este criterio, la fracción de galaxias elípticas está en muy buen acuerdo con la distribución observada en las tres versiones del modelo luego de ser calibrado.

Las distribuciones de $[\alpha / \mathrm{Fe}]$-masa estelar de nuestros modelos son comparados con la relación $[\alpha / \mathrm{Fe}]$-masa dinámica presentada por T10 (contornos de línea azul) y datos de Arrigoni et al. (2010) (cuadrados azules). La línea a rayas azul denota el ajuste lineal a la relación $[\alpha / \mathrm{Fe}]$-masa dinámica de T10, mientras que la línea sólida roja representa el ajuste lineal a la distribución de galaxias del modelo. Es importante señalar que la comparación de cocientes de abundancias obtenida a partir de modelos que incluyen una TH-IGIMF con los datos observados considerados aquí no es estrictamente justa. Los patrones de abundancia observados son obtenidos comparando los rasgos espectrales observados de galaxias con espectros sintéticos de modelos de síntesis de poblaciones. Estos modelos suponen una IMF estelar fija que no es dependiente de la SFR, como la TH-IGIMF. Los modelos de síntesis de poblaciones usados por T10 son aquellos de Thomas \& Maraston (2003), quienes suponen una IMF de Salpeter para calcular los parámetros de las poblaciones estelares. Arrigoni et al. (2010) usan abundancias recalculadas de Trager et al. (2008), quienes también usan los modelos de síntesis de 


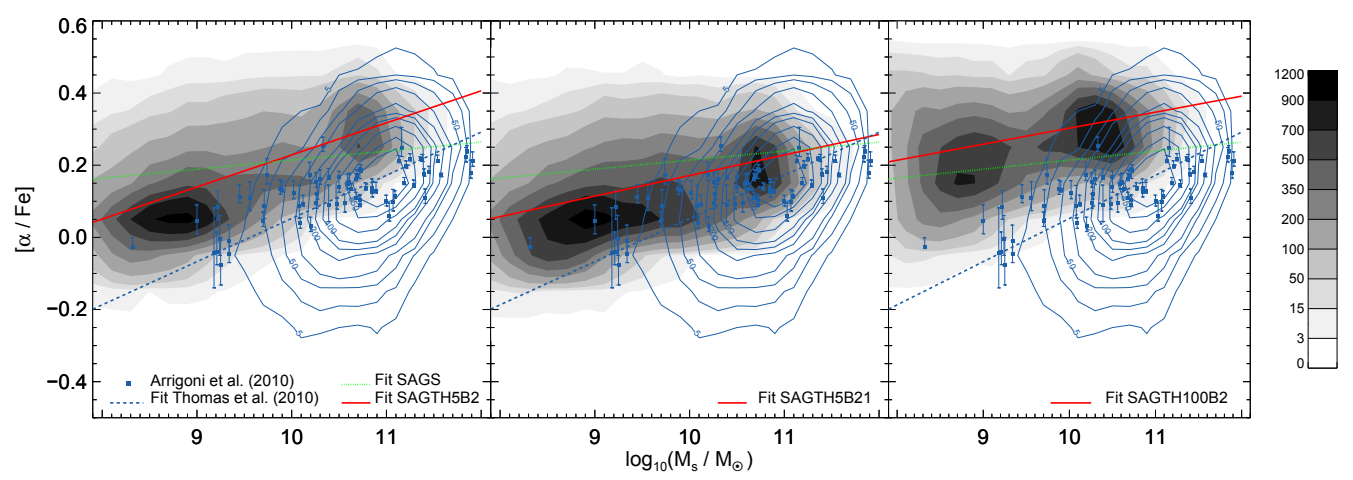

Figura 4.3: Relación $[\alpha / \mathrm{Fe}]-$ masa estelar en modelos SAG1STH5B2 (panel izquierdo), SAG1STH5B21 (panel del medio) y SAG1STH100B21 (panel derecho) representada por contornos de densidad grises; las líneas sólidas rojas son ajustes a las distribuciones de cocientes de abundancias. Los resultados de todos los modelos son comparados con el ajuste a la distribución de $[\alpha / \mathrm{Fe}]$-masa estelar del modelo SAG1S, representado por una línea verde punteada. Los contornos de línea azules muestran la relación trazada por la muestra de galaxias elípticas de T10; el ajuste a la distribución observada de cocientes de abundancia $[\alpha / \mathrm{Fe}]$ en función de la masa está representado por la línea azul a rayas. Los símbolos azules corresponden a datos de Arrigoni et al. (2010). 
poblaciones correspondientes a la IMF de Salpeter. Además, los cocientes de abundancia observados son calculados usando espectros integrados de galaxias en un intervalo de longitud de onda específico. Una comparación justa con los resultados del modelo debería incluir abundancias pesadas por luminosidad en el ancho de banda correspondiente de nuestro modelo. Sin embargo, Thomas (1999) investiga cómo las abundancias pesadas por luminosidad subestiman las abundancias pesadas por masa. Ellos encuentran que la diferencia es despreciable en el caso de una IMF fija. Más tarde, Recchi et al. (2009) encuentran que, usando una IGIMF, la diferencia entre abundancias pesadas por luminosidad y por masa permanece en el orden de 0,1 dex. A la luz de estos resultados, comparamos los datos observados directamente con las abundancias de SAG, que tienen en cuenta la masa total de elementos químicos en estrellas. Teniendo en mente estas inconsistencias, ahora analizamos las características de la relación $[\alpha / \mathrm{Fe}]$-masa estelar que emerge de nuestro modelo semianalítico para diferentes valores de masa mínima de cúmulo estelar inmerso, $M_{\mathrm{ecl}}^{\mathrm{min}}$, y la pendiente de la función de masa de cúmulos embebidos, $\beta$. Considerando los gráficos en la Fig. 4.3, vemos que la TH-IGIMF produce un cambio positivo en la tendencia de cocientes de abundancia $[\alpha / \mathrm{Fe}]$ con la masa estelar (contornos grises y ajustes representados por líneas rojas sólidas) respecto a la obtenida para SAGS, el cual considera una IMF de Salpeter (línea verde punteada). La IMF dependiente de la SFR permite recuperar la pendiente denotada por los datos observacionales. Aunque este es el caso para todos los valores considerados para $M_{\mathrm{ecl}}^{\mathrm{min}} \mathrm{y} \beta$, el acuerdo con la tendencia observacional es particularmente bueno para $M_{\mathrm{ecl}}^{\mathrm{min}}=5 \mathrm{M}_{\odot}$ y $\beta=2$ (panel de la izquierda). La pendiente del ajuste a los cocientes de abundancia $[\alpha / \mathrm{Fe}]$ mostrados por SAGTH5B2 $(a=0,088)$ es mas empinada que aquellas obtenidas de los modelos SAGTH5B21 y SAGTH100B2 $(a=0,0568$ and $a=0,044$, respectivamente). El primero está más cercano a la pendiente del ajuste a los datos de T10 ( $a=0,1184$ ), el cual fue obtenido teniendo en cuenta todas las galaxias de la muestra. El valor de 0,1 dado por T10 corresponde a su submuestra de galaxias rojas. Todos los ajustes de los modelos con distintas combinaciones de parámetros de la TH-IGIMF dan pendientes más cercanas a la observada que el modelo SAGS, que arroja una pendiente de $a=0,025$. Sin embargo, si consideramos los valores de las medianas, correspondientes a los contornos más oscuros, el modelo muestra un exceso de $[\alpha / \mathrm{Fe}]$ para todas las masas de galaxias. Esto está relacionado con una falta de Fe en las galaxias de todos nuestros modelos. La modificación de los tiempos de retardo de SN Ia (Yates et al., 
2013) en la implementación química podría mejorar este aspecto particular. Dejamos este cambio para desarrollos futuros.

Considerando que $M_{\mathrm{ecl}}^{\mathrm{min}}$ y $\beta$ son parámetros libres de la teoría de TH-IGIMF, los resultados que emergen del análisis de la Fig. 4.3 apoyan un valor tan chico como $5 \mathrm{M}_{\odot}$ y 2 para estas cantidades. Como mencionamos en la Sección 8.2 del Capítulo 3, hay evidencia de nubes de formación estelar con masas de hasta $5 \mathrm{M}_{\odot}$ y se observa que la pendiente de la función de masa de cúmulos inmersos es cercana a 2 (Lada \& Lada, 2003). Sin embargo, el valor de esta masa mínima puede cambiar con el redshift, corriéndose a valores más altos debido a condiciones de formación estelar más violentas. Weidner et al. (2013) también encuentran evidencia de que el índice de ley de potencia $\beta$ de la función de masa de cúmulos inmersos decrece cuando crece la SFR. A pesar de ello, hasta ahora no hay restricciones observacionales ni modelo físico que ayude a determinar una variación preferencial en galaxias elípticas.

Para comprender la manera en la cual opera una TH-IGIMF ayudando a lograr los correctos cocientes de abundancia de $[\alpha / \mathrm{Fe}]$ para galaxias de diferente masa, analizamos la evolución de la pendiente media de la TH-IGIMF ( $\left\langle\alpha_{\mathrm{TH}}\right\rangle$, ver ec. 3.16) pesada por la masa estelar formada en cada evento, los cuales están caracterizados por una dada SFR, para galaxias dentro de diferentes intervalos de masa; las galaxias en cada intervalo de masa son seleccionadas de acuerdo a sus masas estelares a $z=0$. Esto es mostrado en la Fig. 4.4 para los modelos SAGTH5B2, SAGTH5B21 y SAGTH100B2 (paneles de la arriba, medio y abajo, respectivamente). La tendencia evolutiva general de $\left\langle\alpha_{\mathrm{TH}}\right\rangle$ es muy similar para todos los modelos, pero los valores de $\left\langle\alpha_{\mathrm{TH}}\right\rangle$ para un intervalo de masa dado a $z=0$ son menores para valores más altos de $M_{\text {ecl }}^{\text {min }}$ y para valores más bajos de $\beta$. En general, a pesar del valor de estos parámetros libres, podemos ver que $\left\langle\alpha_{\mathrm{TH}}\right\rangle$ se vuelve progresivamente más bajo para galaxias más masivas, esto es, los eventos de formación estelar que tienen lugar en galaxias más masivas están caracterizados por IMFs más planas, lo cual refleja que galaxias más masivas tienen SFRs más altas. Estos niveles de tasa de formación estelar y su dependencia con la masa estelar lleva a valores adecuados de la SFR específica (sSFR, por su sigla en inglés) definida como SFR por unidad de masa estelar. Esto es mostrado en el panel interno en el gráfico de arriba, donde podemos ver que la sSFR a $z=0$ decrece con el incremento de la masa estelar, en acuerdo con la tendencia general encontrada por Feulner et al. (2005) para galaxias a redshifts más altos. 

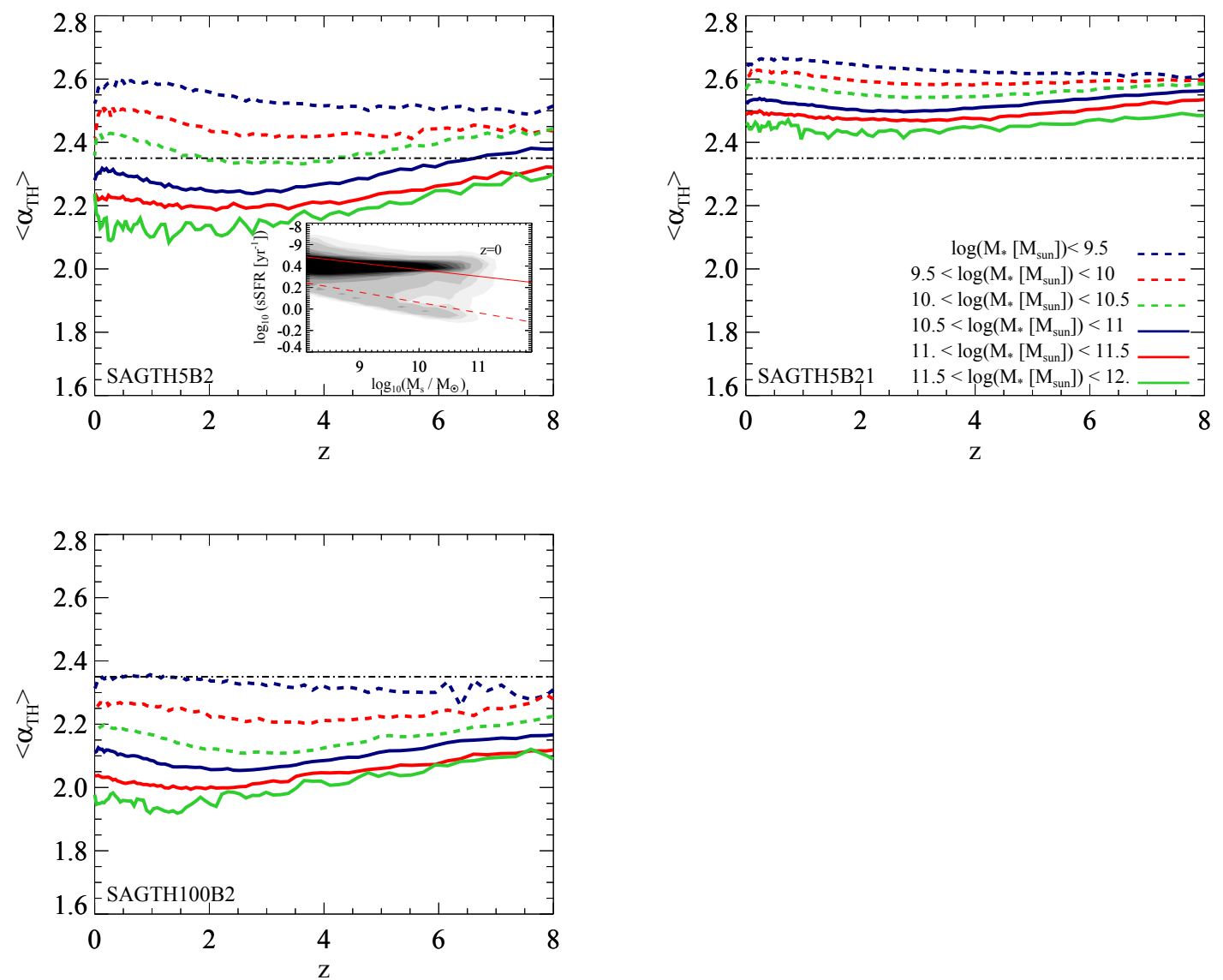

Figura 4.4: Evolución de la pendiente media de la TH-IGIMF $\left(\left\langle\alpha_{\mathrm{TH}}\right\rangle\right.$, ver ec. 3.16) pesada con la masa estelar formada en cada evento, la cual es caracterizada por una dada SFR, para galaxias en diferentes intervalos de masa, como está indicado en las referencias del gráfico; galaxias en cada intervalo de masa son seleccionadas de acuerdo a su masa estelar a $z=0$. Los resultados se muestran para los modelos SAGTH5B2 (panel superior izquierdo), SAGTH5B21 (panel superior derecho) y SAGTH100B2 (panel inferior), caracterizados por los parámetros $M_{\mathrm{ecl}}^{\min }=5 \mathrm{M}_{\odot}$ y $\beta=2 ; M_{\mathrm{ecl}}^{\min }=5 \mathrm{M}_{\odot}$ y $\beta=$ 2,1 y $M_{\text {ecl }}^{\min }=100 \mathrm{M}_{\odot}$ y $\beta=2$, respectivamente. La línea de rayas y puntos horizontal en cada panel representa el valor de la pendiente de la IMF de Salpeter. El gráfico interno en el panel de la izquierda contiene la distribución de la tasa de formación estelar específica (sSFR) a $z=0$ como función de la masa estelar (contornos grises). La líneas sólida y rayada representan el ajuste lineal a la distribución de SSFR para galaxias activas y pasivas, respectivamente; la división satisface la condición definida por Kimm et al. (2011) (ver su ec. 8). 
Los valores más bajos de la pendiente para cada intervalo de masa son alcanzados a épocas más tempranas para galaxias de masas menores, corriéndose desde $1 \lesssim$ $z \lesssim 2$ a $3 \lesssim z \lesssim 4$ para galaxias con masas estelares a $z=0$ de $\sim 5,6 \times 10^{11} \mathrm{M}_{\odot} \mathrm{y}$ $\sim 1,8 \times 10^{10} \mathrm{M}_{\odot}$, respectivamente. La forma de la dependencia de $\left\langle\alpha_{\mathrm{TH}}\right\rangle$ con el redshift para cada intervalo de masa indica que progenitores de menor masa tienen menores niveles de formación estelar y entonces sus eventos de formación están caracterizados por IMFs más empinadas. Así, los progenitores de las galaxias más masivas tienen un valor de $\left\langle\alpha_{\mathrm{TH}}\right\rangle$ a $z \approx 7$ bastante similar al valor correspondiente de galaxias dentro del intervalo de masa $3,1 \times 10^{10} \mathrm{M}_{\odot}<M_{\star}<1 \times 10^{11} \mathrm{M}_{\odot}$ a $z \approx 3$. Es interesante notar que el modelo SAGTH5B2, el cual da una relación $[\alpha / \mathrm{Fe}]-$ masa estelar en mucho mejor acuerdo con las observaciones que los otros dos modelos (SAGTH5B21 and SAGTH100B2), tienen eventos de formación estelar caracterizados por IMFs más empinadas que la IMF de Salpeter para galaxias de baja masa y más planas para galaxias masivas, ya que la discusión general encontrada en la literatura considera que una IMF más plana que Salpeter es necesaria para reproducir la relación $[\alpha / \mathrm{Fe}]$-masa estelar observada (Nagashima et al., 2005; Calura \& Menci, 2009; Arrigoni et al., 2010). Calura \& Menci (2009) proponen que una IMF plana debe ser considerada cuando la SFR de las galaxias excede un valor de $100 \mathrm{M}_{\odot}$. Ellos obtienen una pendiente positiva de la relación $[\alpha / \mathrm{Fe}]-\sigma$, aunque la dependencia se vuelve plana para galaxias con altas dispersiones de velocidades y también para galaxias con baja dispersión de velocidades, cuando no se acciona ningún brote estelar por interacciones (ver su figura 20). Este resultado, en contraste con los resultados en este trabajo, resalta la importancia en la progresividad de la variación en la pendiente de la IMF en galaxias, avalada por un modelo testeado empíricamente de IMF para toda la galaxia como la TH-IGIMF.

A partir de nuestra investigación, encontramos que el aspecto clave para lograr la correcta dependencia de los cocientes de abundancias de $[\alpha / \mathrm{Fe}]$ con la masa estelar es la diferencia relativa de la pendiente de la IMF para galaxias con diferentes masas. En otras palabras, la pendiente de la IMF misma no es fundamental mientras se vuelva progresivamente más plana para galaxias más masivas.

En los modelos con una TH-IGIMF caracterizada por valores más altos de $M_{\mathrm{ecl}}^{\min }$ (SAGTH100B2) y un valor más alto de $\beta$ (SAGTH5B21), la diferencia relativa de $\left\langle\alpha_{\mathrm{TH}}\right\rangle$ para intervalo de masa distintos se preserva, dando lugar así a una relación $[\alpha / \mathrm{Fe}]$-masa estelar con una pendiente positiva. Sin embargo, la diferencia relativa entre diferentes in- 
tervalos de masa en el caso de un $\beta$ más alto es más suave. En el caso de $M_{\text {ecl }}^{\min }$ más altos, las pendientes son más planas que Salpeter para todos los bines de masa, y la pendiente resultante de la relación $[\alpha / \mathrm{Fe}]$-masa estelar es más plana que en SAGTH5B2, apoyando la noción de que el valor absoluto de las pendientes medias no son tan importantes como el diferente comportamiento de las galaxias con diferentes masas, y señalando en la dirección de que galaxias de masa baja deberían tomar pendientes en su IMF más empinadas que Salpeter, mientras que galaxias de mayor masa deberían tomar pendendientes más planas que la de Salpeter para alcanzar las abundancias químicas correctas. Esto también explica las sobre-abundancias de elementos $\alpha$ en el modelo SAGTH100B2; virtualmente, todos los eventos de formación estelar contienen un número grande de estrellas masivas con masas mayores a $8 \mathrm{M}_{\odot}$, estableciéndose una mayor producción de SN II.

Nuestros resultados nos permiten restringir los posibles valores de los parámetros libres involucrados en la teoría de la TH-IGIMF, esto es, la masa mínima de cúmulo estelar inmerso, apoyando un valor tan chico como $M_{\mathrm{ecl}}^{\mathrm{min}}=5 \mathrm{M}_{\odot}$, y la pendiente de la función de masa de cúmulos inmersos $\beta$, para el cual la mejor opción parece ser $\beta=2$.

\section{Brotes extendidos y escala temporal de explosión de SNs}

Para poder entender el patrón particular desarrollado por los cocientes de abundancia $[\alpha / \mathrm{Fe}]$ de galaxias elípticas deben ser analizados la duración de los brotes estelares en las galaxias en diferentes intervalos de masa y el tiempo de vida de los progenitores de los diferentes tipos de SN.

\subsection{Brotes extendidos: diferentes suposiciones sobre la duración inicial del brote}

En la sección 9 del Capítulo 3, introdujimos la implementación de brotes extendidos en el modelo SAG. Ahora analizamos los resultados y sus implicaciones en el enriquecimiento químico de las galaxias elípticas. También investigamos el rol de los brotes extendidos en el desarrollo de la relación $[\alpha / \mathrm{Fe}]$-masa estelar. La Fig. 4.5 presenta la 
duración media de los brotes estelares que tienen lugar en galaxias en diferentes intervalos de masa como función del redshift; las galaxias se agrupan de acuerdo a sus masas estelares a $z=0$. El redshift indica el tiempo en el cual el brote fue disparado. Consideramos aquí sólo brotes que terminaron como resultado de la consumisión del gas frío del bulbo. Hay varios aspectos para notar en este gráfico. Primero, la escala de tiempo media estimada de los brotes (líneas finas) crece a medida que el redshift decrece, con una tendencia muy similar para galaxias de diferente masa estelar. Esto surge por el crecimiento natural de las galaxias que están caracterizadas por tiempos dinámicos más largos a épocas más recientes. Segundo, la duración real de los brotes (líneas gruesas) son más cortas que las escalas de tiempo estimadas de brotes para galaxias en todo el intervalo de masas. Esto es un resultado de la competición de varios procesos. La reserva de gas frío del bulbo es común a todos los brotes que tienen lugar simultáneamente en una dada galaxia. Estos brotes pueden haber sido disparados en diferentes momentos, contribuyendo inicialmente a incrementar la reserva de gas frío del bulbo. Esta reserva es, a su vez, disminuida por retroalimentación de SNs de estrellas en el bulbo ya formadas en brotes anteriores finalizados o en curso. El gas frío del bulbo también es reducido por el crecimiento gradual del agujero negro central. Estos procesos hacen que los brotes alcancen su final antes que lo estimado originalmente; esta diferencia es más pronunciada para galaxias menos masivas para las cuales el efecto acumulativo de la retroalimentación por SN tiene un impacto más fuerte.

Considerando brotes disparados a $z \approx 0$ en el modelos SAGS (panel superior), encontramos que su duración media real son del orden de $9 \times 10^{7} \mathrm{yr}, 2 \times 10^{8}$ yr, y $5 \times 10^{8} \mathrm{yr}$ para masas estelares en el intervalo $10^{8}-10^{9} \mathrm{M}_{\odot}, 10^{9}-10^{10} \mathrm{M}_{\odot}$, y mayores a $10^{10} \mathrm{M}_{\odot}$, respectivamente. Estos valores pueden ser comparados con las escalas de tiempo exponenciales de brotes a $z=0$ dadas por el modelo semianalítico utilizado por Granato et al. (2000) (ver su fig. 6), el cual se supone que se compara con la escala de tiempo dinámico de un esferoide formado en una fusión mayor; este proceso es el único canal de formación de bulbo que ellos consideran. Sus valores de escalas de tiempo de brotes estelares van desde $5 \times 10^{6}$ yr to $1 \times 10^{8}$ yr para galaxias en el intervalo $10^{8}-3 \times 10^{11} \mathrm{M}_{\odot}$. La duración media estimada de brotes en el modelo SAGS son del mismo orden de magnitud para todas las galaxias y son más largas que la escala de tiempo exponencial de brote obtenida por Granato et al. (2000). 

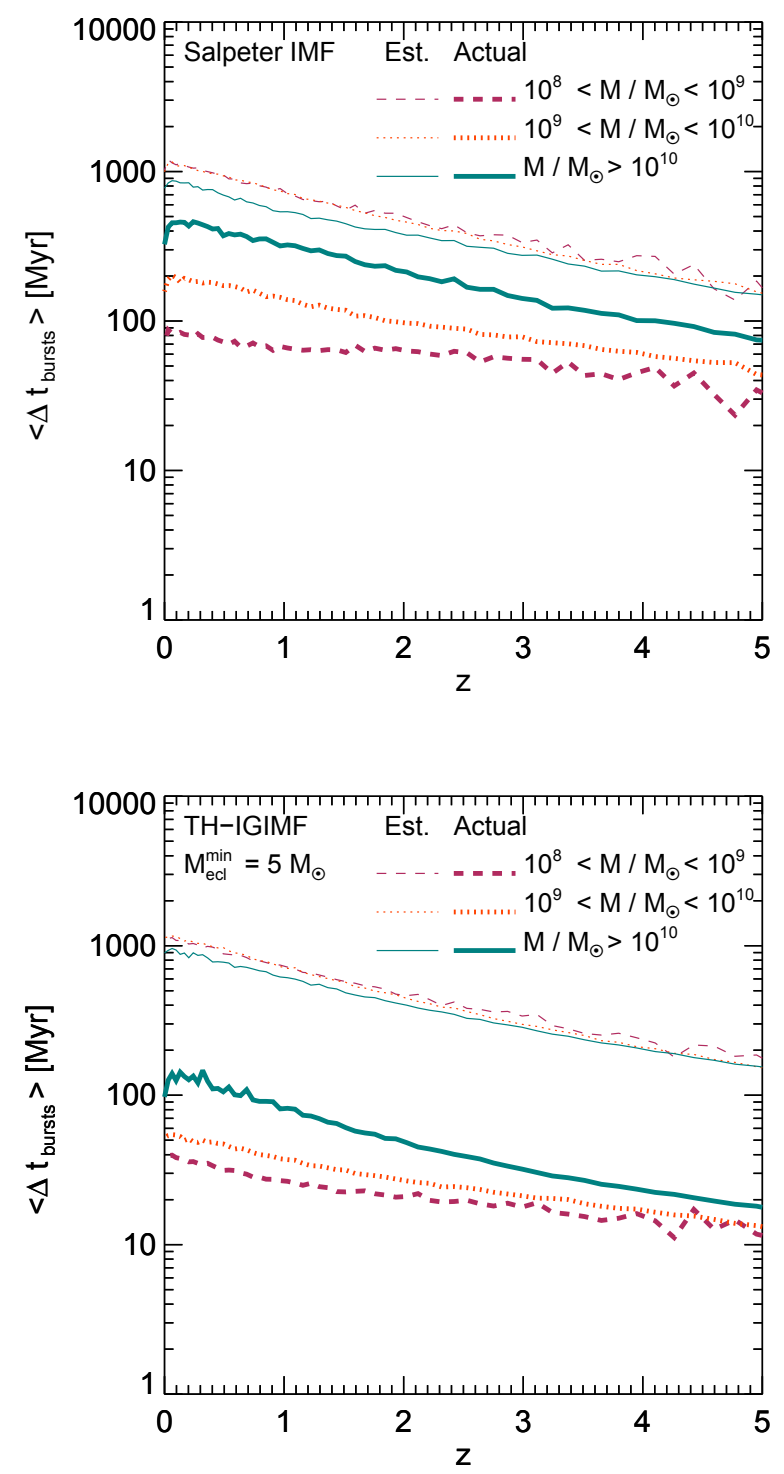

Figura 4.5: Panel superior: Duración media de brotes como función del redshift para galaxias generadas por el modelo SAGS en diferentes intervalos de masa, tal como está indicado en la etiqueta; las galaxias son agrupadas de acuerdo a su masa estelar a $z=0$. Las duraciones medias de brotes y las duraciones reales están representadas por líneas finas y gruesas, respectivamente. Panel inferior: Igual al panel superior, pero para el modelo SAGTH5B2. 
El hecho de que, sin importar el redshift en el cual los brotes son disparados, la duración media de los brotes se incrementa con la masa estelar, contribuye a explicar la pendiente tímidamente positiva en el modelo SAGS. Sin embargo, enfatizamos aquí que la escala de tiempo de formación galáctica que, de acuerdo a numerosos trabajos, se supone que conduce a la relación $[\alpha / \mathrm{Fe}]$-masa estelar en galaxias elípticas, no está ligada a la duración de brotes individuales. Esa escala es una integración sobre tiempos cósmicos de múltiples eventos de formación estelar.

El argumento de escalas de tiempo que explica el comportamiento de la relación $[\alpha / \mathrm{Fe}]$-masa estelar en el modelo es válido mientras consideremos una IMF universal. Una IMF variable, cuya pendiente depende de la SFR da diferentes números relativos de SN Ia y progenitores de SN II para cada evento de formación estelar. Este aspecto juega un importante rol en la construcción de la relación $[\alpha / \mathrm{Fe}]$-masa estelar, como vimos en la sección 4.1. La dependencia con el redshift de brotes estelares en el caso del modelo SAGTH5B2 (panel inferior de la Fig. 4.5) es bastante similar al modelo con una IMF de Salpeter, pero la duración del brote es un factor 2 más corto.

La duración más corta de los brotes en SAGTH5B2 es explicada si tenemos en cuenta que las pendientes medias de la IMF, $\left\langle\alpha_{\mathrm{TH}}\right\rangle$, se vuelven progresivamente mayores que la pendiente de una IMF de Salpeter a medida que consideramos galaxias menos masivas (ver panel izquierdo de la Fig. 4.4). Este hecho conduce a una baja tasa de SN II para galaxias de baja masa que tienen que ser compensadas por valores grandes de la eficiencia de retroalimentación por $\mathrm{SN}$ para el disco y el bulbo ( $\varepsilon$ y $\varepsilon_{\text {bulbo, }}$, respectivamente), que son parámetros libres del modelo, para reproducir el extremo débil de la LFs. Estos valores son listados en la tabla 4.2; podemos ver que son más grandes que los correspondientes valores para el modelo calibrado SAGS (tabla 4.1), especialmente $\varepsilon_{\text {bulbo }}$.

Entonces, las galaxias en el modelo SAGTH5B2 sufren, en promedio, un efecto mucho más fuerte de retroalimentación por SN proveniente de estrellas en el bulbo que las galaxias en el modelo SAGS. Este efecto más fuerte contribuye a consumir más rápidamente la reserva de gas frío del bulbo para brotes estelares, reduciendo considerablemente su duración real. Contrario a lo que es obtenido del modelo SAGS, la duración real del brote obtenida para el modelo SAGTH5B2 y su dependencia con la masa estelar está en buen acuerdo con la escala de tiempo exponencial de brotes a $z=0$ obtenidas por Granato et al. (2000), cuyos resultados han sido descriptos anteriormente en esta 
sección. Este buen acuerdo es logrado por la consideración de una TH-IGIMF en nuestro modelado. Los valores obtenidos de nuestro modelo para las galaxias más masivas también son consistentes con los resultados para simulaciones numéricas dados por Di Matteo et al. (2008), quien encontró que la actividad de brotes estelares activados por fusiones tienen una duración típica de unos cuantos $10^{8} \mathrm{yr}$. Luego de haber analizado las características de las duraciones medias estimadas y reales de brotes resultantes del modelado adoptado en este trabajo, investigamos el impacto de una suposición diferente para la estimación inicial de la escala de tiempo en la duración media real de los brotes estelares y en la relación $[\alpha / \mathrm{Fe}]$-masa estelar. La combinación de esta cantidad con la cantidad de gas frío disponible define su SFR. Así, los modelos de formación de galaxias con una IMF dependiente de la SFR, como los modelos con TH-IGIMF analizados aquí, van a ser afectados por la elección de este valor inicial. La retroalimentación por $\mathrm{SN}$ y el crecimiento de $\mathrm{BH}$ determinan la duración real del brote, pero mantienen la SFR inalterada.

Consideramos el modelo SAGTH5B2 y modificamos la estimación de la duración inicial del brote, adoptando el caso más simple en el cual todas las galaxias tienen el mismo valor inicial estimado para la duración de los brotes, $\Delta \mathrm{T}_{\text {burst }}^{\text {estimated }}=50 \mathrm{Myr}$. La duración media estimada y la duración real de los brotes como función del redshift para este modelo modificado son mostrados en la Fig. 4.6 para galaxias en diferentes intervalos de masa. La línea fina denota el valor estimado de duración de brote, el cual es idéntico para todas las galaxias a todos los tiempos. Los valores de duración real media son caracterizados por una evolución con el redshift que es bastante similar para todas las galaxias, y sólo muestran una dependencia débil con la masa estelar a altos redshifts. Estos valores se acercan a los inicialmente estimados a medida que decrece el redshift, indicando que el pozo de potencial de las galaxias se vuelve mayor a redshifts más chicos con el consecuente menor efecto de la retroalimentación por SNs. Como las escalas de tiempo inicialmente estimadas son más pequeñas que aquéllas estimadas considerando las escalas de tiempo dinámico del disco (ver el panel inferior de la Fig. 4.5), las duraciones reales de brote resultantes también son menores.

La Fig. 4.6 muestra el impacto de estos brotes más cortos y su evolución en la relación $[\alpha / \mathrm{Fe}]$-masa estelar obtenida para el modelo modificado SAGTH5B2. La línea sólida roja denota el ajuste lineal a la distribución de cocientes de abundancia $[\alpha / \mathrm{Fe}]$ con la masa estelar. Ésta tiene una pendiente de $a=0,076$, un poco más baja que aquélla 

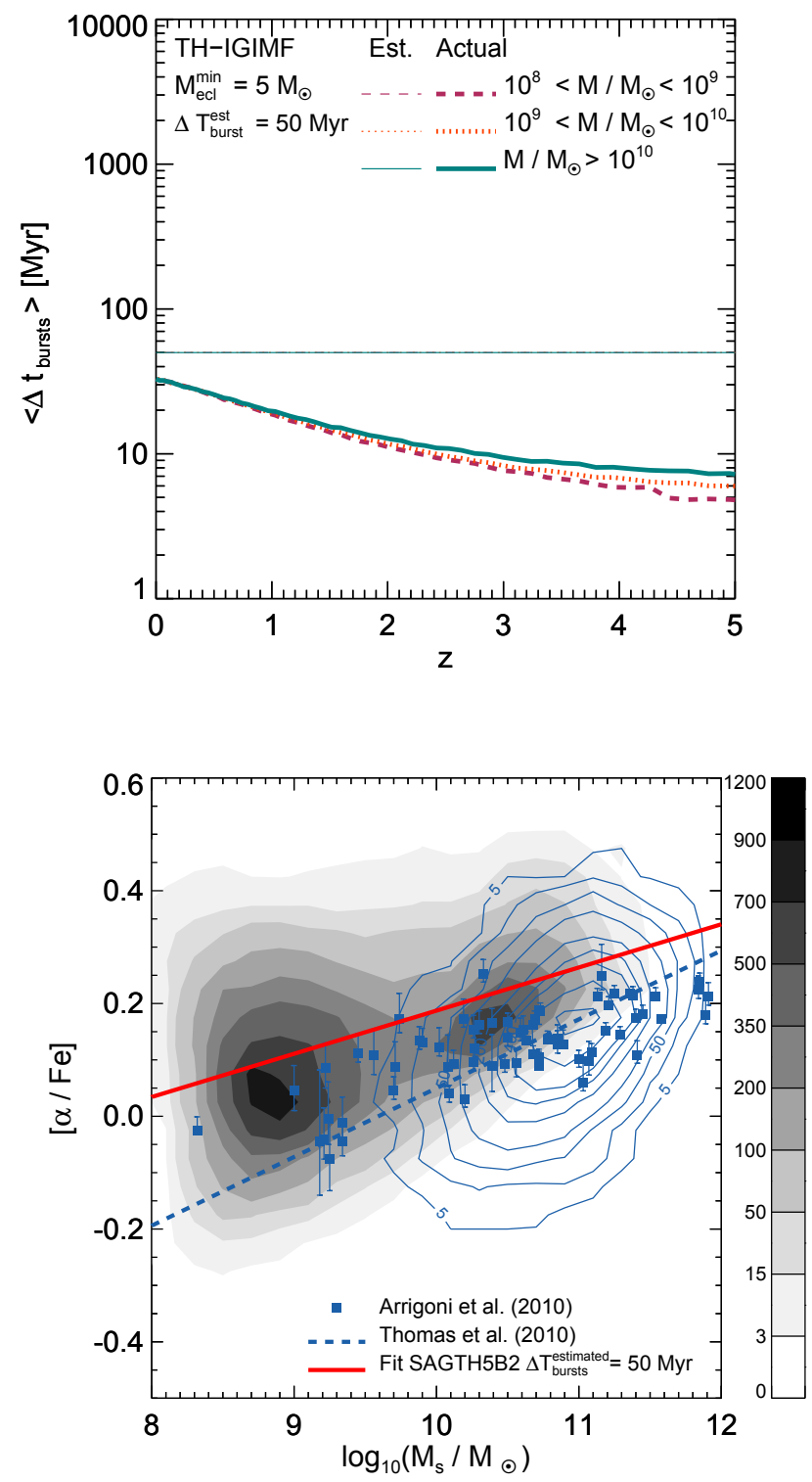

Figura 4.6: Panel superior: Duración media de los brotes finalizados como función del redshift en el cual fueron disparados para galaxias en diferentes intervalos de masa para el modelo SAGTH5B2, como está indicado en las etiquetas; las galaxias son seleccionadas de acuerdo a su masa estelar a $z=0$. Se supone un valor fijo de $50 \mathrm{Myr}$ para la estimación inicial de escala de tiempo de brote para todas las galaxias. Panel inferior: Relación $[\alpha / \mathrm{Fe}]$-masa estelar para el modelo SAGTH5B2 con un valor de brote inicial estimado de $50 \mathrm{Myr}$ para todas las galaxias. 

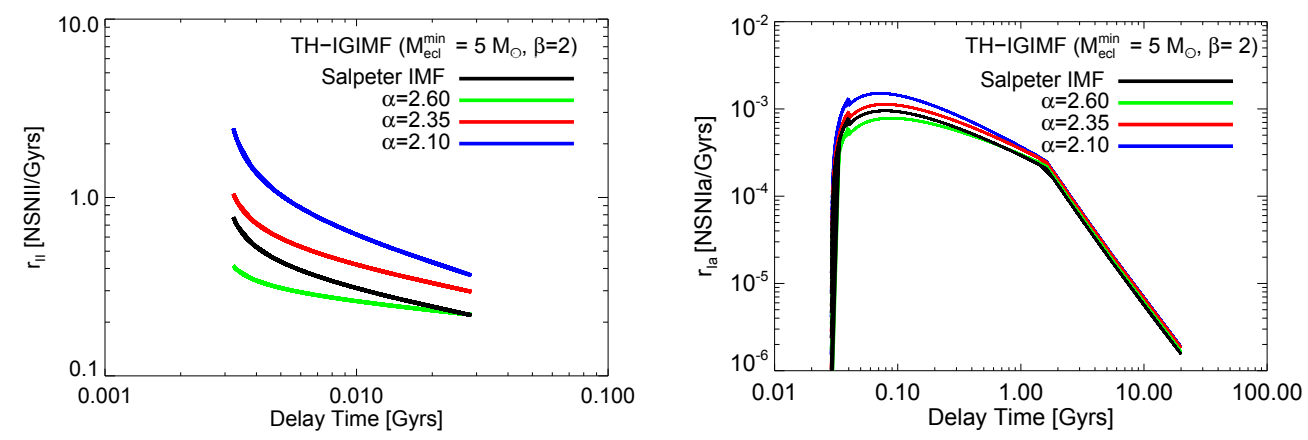

Figura 4.7: Tasas de SNs II y SN Ia como función de sus tiempos de retardo para la explosión (delay times) para tres valores posibles de $\alpha_{\mathrm{TH}}$ para una TH-IGIMF con $M_{\mathrm{ecl}}^{\min }=5 \mathrm{M}_{\odot} \mathrm{y} \beta=2$.

correspondiente al modelo SAGTH5B2 que usa el tiempo dinámico como estimación inicial de la duración del brote $(a=0,088)$. Es importante notar que estamos usando el mismo conjunto de parámetros que aquéllos correspondientes al modelo SAGTH5B2. En definitiva, diferentes suposiciones sobre la duración inicial de los brotes no producen un cambio significativo en el patrón de las abundancias químicas de las galaxias elípticas. Estos resultados proveen soporte adicional a nuestras conclusiones de que el principal responsable para el desarrollo de la tendencia adecuada de cocientes de abundancia $[\alpha / \mathrm{Fe}]$ con la masa estelar es una TH-IGIMF dependiente de la SFR, con valores preferidos de sus parámetros libres $M_{\mathrm{ecl}}^{\min }=5 \mathrm{M}_{\odot} \mathrm{y} \beta=2$.

\subsection{Escala temporal de explosión de SNs}

El panel izquierdo de la Fig. 4.7 muestra las tasas de SNs CC (núcleo colapsante, por sus siglas en inglés) como función de sus tiempos de retardo para la explosión o delay times, esto es, la escala temporal de las explosiones. Líneas de diferentes colores corresponden a las tasas obtenidas para una TH-IGIMF con $M_{\mathrm{ecl}}^{\min }=5 \mathrm{M}_{\odot}$ y $\beta=2$ y tres diferentes valores posibles de $\alpha_{\mathrm{TH}}$. Como es de esperarse, IMFs más planas dan lugar a mayores tasas de SNs II. Estas tasas son comparadas con aquéllas obtenidas de una IMF de Salpeter (línea negra). Las tasas de SN Ia correspondientes a estos cuatro casos son presentadas en el panel inferior de la Fig. 4.7.

Como mencionamos en la sección 8.1 la tasa de SNs Ia es estimada de acuerdo 
al formalismo descripto en Lia et al. (2002), que supone que una SN Ia se origina en sistemas binarios (Greggio \& Renzini, 1983). La fracción de estos sistemas binarios, $A_{\text {bin }}$, es uno de los parámetros libres de SAG. Para una IMF supuesta, el valor adoptado por $A_{\text {bin }}$ determina la tasa de SNs Ia. En el caso de una IMF Universal, como la IMF de Salpeter adoptada en el modelo SAGS, el valor de $A_{\text {bin }}$ está ligado a esa IMF, restringido por el requerimiento de reproducir la evolución observada de las tasas de SN Ia. Para un modelo con TH-IGIMF, la pendiente de la IMF para masas estelares más grandes que $1,3 M_{\odot}$ (ver ec. 3.16) puede variar dentro de un intervalo, de acuerdo a la SFR de un dado evento de formación estelar. Sin embargo, nosotros consideramos una única fracción de sistemas binarios sin importar la pendiente de la IMF, $\alpha_{\mathrm{TH}}$. Así, las tasas de SNs Ia para cada población estelar difieren para diferentes valores de $\alpha_{\mathrm{TH}}$, como puede verse en el panel inferior de la Fig. 4.7, donde las tasas son dadas como una función de los tiempos de retardo de SNs Ia, los cuales son establecidos por el tiempo de vida de la estrella secundaria del sistema binario. Los valores más bajos (altos) de $\alpha_{\mathrm{TH}}$ (IMF más planas, empinadas), determinadas por los eventos de formación estelar con SFR más altas (bajas), dan lugar a tasas de SNs Ia más altas (bajas). El valor adoptado por $A_{\text {bin }}$ en el proceso de calibración es el resultado de mantener el balance entre la tasa de SNs Ia resultantes para todos los valores posibles de $\alpha_{\mathrm{TH}}$ que pueden ocurrir en todos los eventos de formación estelar tales que el comportamiento promedio de la evolución de las tasas de SNs Ia para toda la población de galaxias emule la evolución observada.

La Figura 4.8 muestra las tasas de SNs calculadas como función del redshift para los modelos SAGS (línea a rayas) y SAGTH5B2 (línea sólida). Los datos observacionales corresponden a la compilación de Melinder et al. (2012). La evolución en las tasas de SNs II son mostradas en el panel superior. Casi no surgen diferencias en los resultados de ambos modelos calibrados. Las tasas observadas muestran una dicotomía a bajo redshift; mientras los trabajos de Botticella et al. (2012) y Mattila et al. (2012) estiman tasas de SNs II de casi $2\left(10^{-4}\right) \mathrm{yr}^{-1} \mathrm{Mpc}^{-3} h_{70}^{3}$, los trabajos de Cappellaro et al. (1999) y Li et al. (2011) dan tasas de SNs II del orden de $0,6\left(10^{-4}\right) \mathrm{yr}^{-1} \mathrm{Mpc}^{-3} h_{70}^{3}$. Las salidas de los modelos son consistentes con los valores más altos de tasas de SNs II a $z=0$. Las tasas de SN Ia mostradas en la Fig. 4.7 para diferentes valores de $\alpha_{\mathrm{TH}}$ son comparados con aquéllas que emergen de una IMF de Salpeter. Podemos ver que para un valor de $\alpha_{\mathrm{TH}}$ igual a la pendiente de la IMF de Salpeter, la TH-IGIMF da valores más altos de SNs Ia, ya que las diferencias entre ambas IMF para masas estelares menores que $1,3 \mathrm{M}_{\odot}$ llevan 

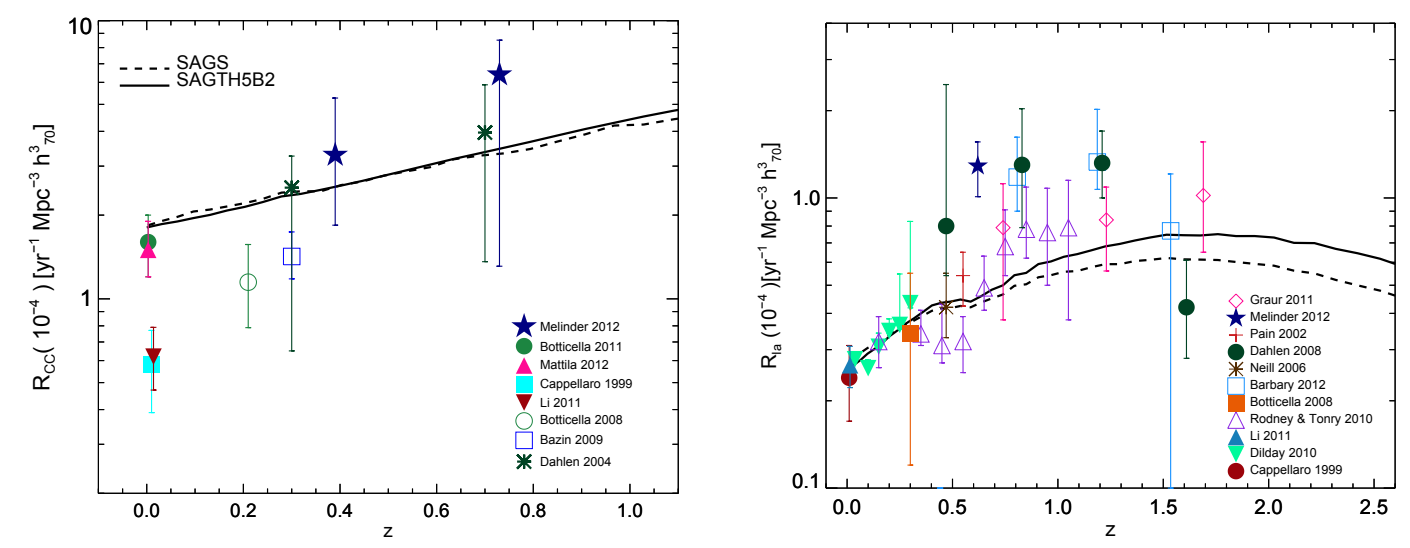

Figura 4.8: Evolución de tasas de SNs II (panel izquierdo) y tasas de SNs Ia (panel derecho) dada por los modelos SAGS (línea a rayas) y SAGTH5B2 (línea sólida), comparadas con la compilación de determinaciones observacionales de Melinder et al. (2012).

a diferentes valores de normalización. Una vez que ambos modelos SAGS y SAGTH5B2 han sido calibrados, las tasas de SNs Ia son similares en los dos modelos, como puede verse de la comparación de líneas de rayas y sólidas en la Fig. 4.8.

Las SNs II en el modelo semianalítico tienen tiempos de retardo entre 2 y $30 \mathrm{Myr}$ (panel superior de la Fig. 4.7), suficientemente cortos para las estrellas que se forman en un brote para encerrar la mayoría de los elementos $\alpha$ producidos durante las explosiones, ya que la duración real de los brotes tienen escalas de tiempo mayores, del orden de las decenas de Myr (ver Fig. 4.5). Por otro lado, los tiempos de retardo de SNs Ia están en el intervalo comprendido entre las decenas de Myr y algunos Gyr, con la mayoría de las SNs Ia que tienen tiempos de retardo entre $20 \mathrm{Myr}-2 \mathrm{Gyr}$ con un pico en aproximadamente 100 Myr. Esta escala de tiempo es del orden del valor máximo de la duración de los brotes estelares, por lo tanto muchos brotes no van a formar estrellas altamente enriquecidas con el principal producto de las SNs Ia, que es el hierro. Sin embargo, ya que los brotes no consumen el gas frío del bulbo instantáneamente, es frecuente que varios brotes coexistan en la misma galaxia y que un brote disparado tardíamente pueda formar estrellas ricas en hierro a partir del gas contaminado por una generación previa de estrellas formadas en el bulbo. Entonces, el cociente de abundancias $[\alpha / \mathrm{Fe}]$ final de una galaxia elíptica es el resultado de la combinación de la duración de los brotes, los tiempos de retardo de SNs y la escala de tiempo de formación de las galaxias. La última 
es analizada en detalle para ambos modelos SAGS and SAGTH5B2 en la Sección 6.

\subsection{Otras pruebas: cocientes $M / L$ y funciones luminosidad}

Matteucci (1994) sugirieron que la hipótesis de una IMF variable para explicar la relación $[\alpha / \mathrm{Fe}]-$ masa estelar en galaxias elípticas debería ser probada en las luminosidades y cocientes de masa-luminosidad $(M / L)$.

En una serie de trabajos, los miembros de la colaboración ATLAS3D estudiaron las propiedades de una muestra de 260 galaxias de tipo temprano (see Cappellari et al., 2011). En el trabajo XV (Cappellari et al., 2013) y XX (Cappellari et al., 2013), ellos se concentran en el plano de masa $\left(\mathrm{M}, \sigma_{e}, R_{e}\right)$, sus proyecciones y la distribución de propiedades de las galaxias en los mismos. Ellos derivan las masas totales (bariónica más materia oscura) dinámicas y cocientes masa-luminosidad ( $\left.M_{\mathrm{JAM}}, M / L_{J A M}\right)$ dentro de una esfera de 1 radio efectivo de galaxias de tipo temprano en su muestra. Ellos señalan que esta cantidad se asemeja y es directamente comparable a la masa estelar total usada en otros estudios, dado que la fracción calculada de MO dentro de ese radio es usualmente baja, $13 \%$ en promedio. Ellos eligen la banda $r$ para medir luminosidades. Vale la pena notar que estas cantidades no están afectadas por suposiciones de la IMF de las galaxias, porque están derivadas usando consideraciones dinámicas y no involucran modelos de síntesis de poblaciones estelares. En la Figura 4.9 comparamos el cociente masa-luminosidad como función de la masa estelar en galaxias elípticas resultantes de nuestro modelo con IMF de Salpeter (panel superior) y SAGTH5B2 (panel inferior), con los datos extraídos de la Tabla 1 de Cappellari et al. (2013). Para este propósito, consideramos la masa estelar de las galaxias dividida por la luminosidad total en la banda- $r$. El error a $1 \sigma$ de las estimaciones de M/L de Cappellari et al. (2013) es de 0.027 dex. Podemos ver que en el caso de nuestra mejor elección de los parámetros de la TH-IGIMF, esto es, $\beta=2$ y $M_{\mathrm{ecl}}^{\mathrm{min}}=5$, el punto de cero de la relación es mejor reproducido que para el caso de SAGS, con un buen acuerdo con los cocientes M/L observados en un amplio intervalo de masas estelares. El apartamiento de las observaciones ocurre para masas menores a $2 \times 10^{10} \mathrm{M}_{\odot}$, para las cuales las pendientes de la IMF son más empinadas que la pendiente de una IMF de Salpeter (ver Fig. 4.4). La forma de los cocientes de $M / L$ es similar para ambos modelos SAGS y SAGTH5B2, con valores menores para el último caso, y una dispersión más alta hacia valores más bajos, como resultado 
de los procesos de calibración que regulan de diferente manera la formación estelar y la retroalimentación energética y química. Esto es, parece que la TH-IGIMF ayuda a recobrar los valores correctos de los cocientes de $M / L$ para galaxias masivas, pero no puede resolver el problema del exceso a masas más bajas con respecto a las observaciones, el cual está presente para cualquier IMF. Este resultado apoya la hipótesis de una TH-IGIMF en galaxias elípticas.

Realizamos una prueba más a la población de galaxias. Comparamos los resultados de los modelos SAGS y SAGTH5B2 con la LF observada en la banda-r de Blanton et al. (2005), usada como restricción para calibrar el modelo. Las LFs resultantes de ambos modelos se muestran en la Fig. 4.10. Como puede verse, no hay mayores diferencias entre ellas y el acuerdo con la LF observada es bastante bueno. Un pequeño exceso de galaxias luminosas está presente en ambos modelos, siendo más notorio en el modelo SAGS. La supresión de formación estelar en las galaxias más masivas no puede ser lograda con el presente modelado de retroalimentación de AGN. En lugar de esto, un mejor acuerdo con las observaciones puede ser obtenido si los brotes durante fusiones de galaxias son disparados sólo cuando la galaxia remanente se vuelve inestable, como es propuesto por Padilla et al. (2013). La LF junto con los cocientes M/L representan una buena prueba a la implementación de una IMF variable, dado que diferentes poblaciones estelares están caracterizados por señales únicas en su luz que deben ser restringidas junto con la relación $[\alpha / \mathrm{Fe}]$-masa para obtener resultados consistentes en las propiedades de las galaxias cuando se modifica una función de distribución importante como lo es la IMF de las estrellas.

\section{6. $[\alpha / \mathrm{Fe}]-m a s a$ estelar: influencia de las escalas de tiem- po de formación}

En este capítulo hemos demostrado que se necesita de una TH-IGIMF para reproducir la tendencia de cocientes de abundancia de $[\alpha / \mathrm{Fe}]$ con la masa estelar mostrada por galaxias elípticas. Sin embargo, un aspecto también considerado para explicar la construcción de esta relación son las escalas de tiempo de formación de las galaxias. A través de este argumento, usualmente se sostiene que las galaxias más masivas son formadas en una escala de tiempo menor que las galaxias menos masivas. (e.g. Thomas 

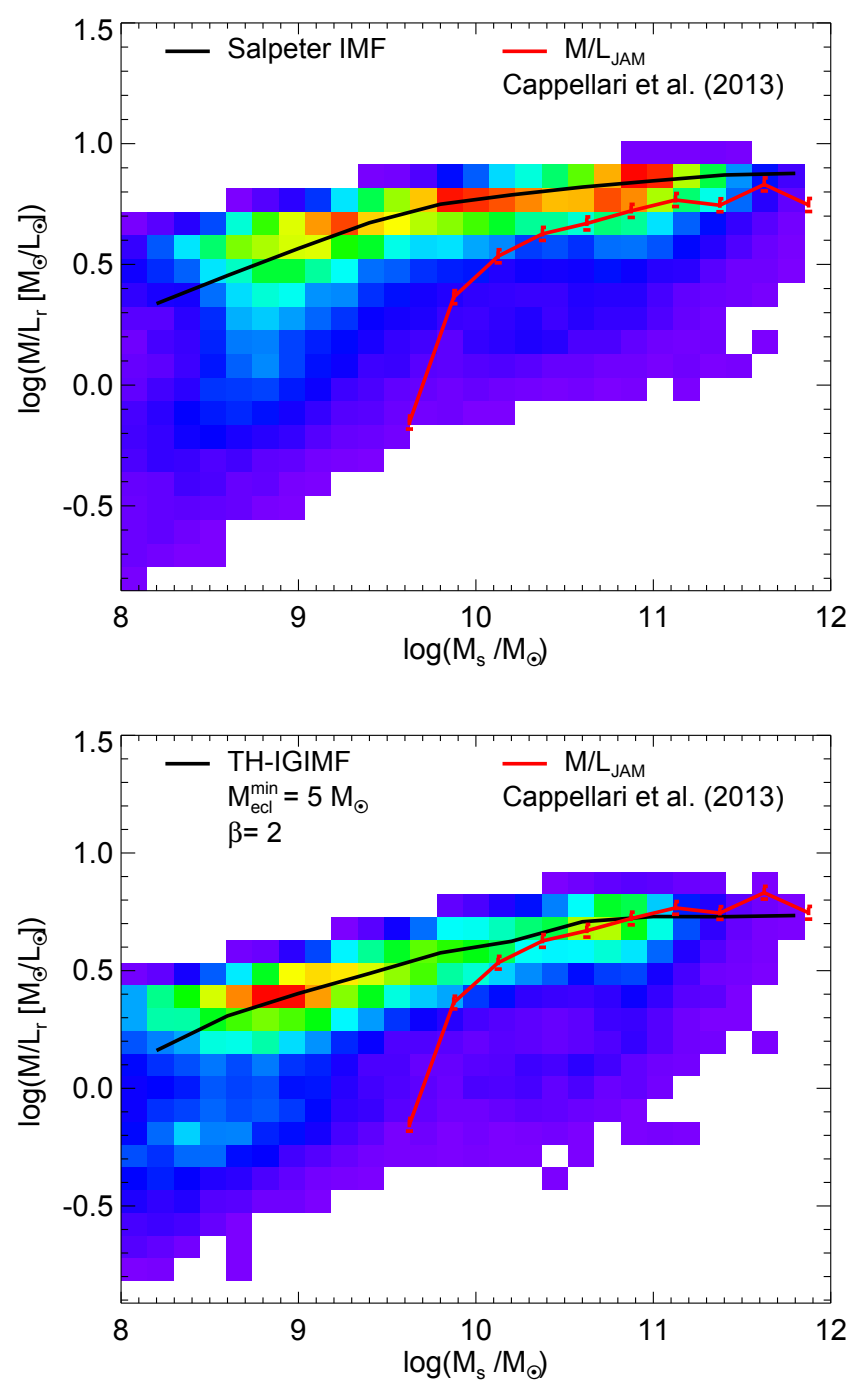

Figura 4.9: Cocientes masa-luminosidad como función de la masa estelar de galaxias elípticas. Los resultados de los modelos SAGS and SAGTH5B2 son mostrados en los paneles superior e inferior, respectivamente. Los mismos son comparados con determinaciones observacionales de Cappellari et al. (2013). 


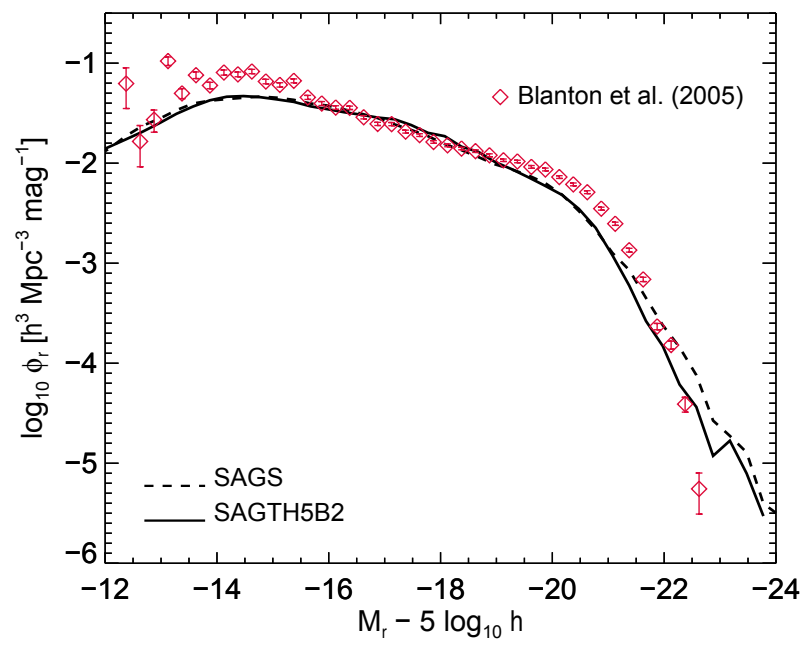

Figura 4.10: Función de luminosidad en la banda- $r$ de la población de galaxias generada por los modelos SAGS (línea a rayas) y SAGTH5B2 (línea sólida) comparadas con las determinaciones observacionales de Blanton et al. (2005) (símbolos abiertos con barras de error).

et al., 2005; Recchi et al., 2009). En este sentido, esta relación es usualmente considerada como otra razón del downsizing. La evidencia observacional indica que las galaxias elípticas reúnen la mayor parte de su masa a alto redshift. Una técnica común utilizada en estos días es la arqueología estelar (Thomas et al., 2005; Neistein et al., 2006). En esta línea de trabajo se miden índices espectrales y son usados para calcular parámetros de poblaciones estelares de galaxias observadas con modelos de síntesis de poblaciones. Un resultado común de aplicar este método es que las poblaciones estelares en galaxias masivas resultan más viejas que en sus contrapartes menos masivas, sin importar la morfología de la galaxia (Panter et al., 2007). Además, las galaxias elípticas presentan las poblaciones estelares más viejas, dado que muestran poca o ninguna actividad de formación estelar, aunque T10 encuentran que aproximadamente 10 por ciento de su muestra de elípticas están formando estrellas activamente y definen un pico de edades jóvenes en su distribución de edades. Neistein et al. (2006) encontraron que este aspecto del comportamiento de downsizing puede ser explicado naturalmente en el marco del agrupamiento jerárquico en términos de los progenitores de los halos principales, definiendo una masa mínima de progenitores que son capaces de formar estrellas. Sin embargo, otros aspectos también considerados como facetas del downsizing como el co- 
rrimiento con el tiempo en la masa característica de las galaxias formadoras de estrellas (Cowie \& Barger, 2008), o el downsizing quemo-arqueológico (Fontanot et al., 2009) no pueden ser explicados con esta línea de razonamiento. Aquí, nos estamos enfocando en el downsizing quemo-arqueológico. El objetivo de esta sección es analizar las escalas de tiempo de formación de las galaxias de nuestra muestra para determinar si las mismas son responsables parcialmente en conducir a una pendiente positiva en la relación $[\alpha / \mathrm{Fe}]$-masa estelar, o no.

Para cada galaxia seleccionada de acuerdo a su masa estelar a $z=0$, definimos la escala de tiempo de formación, $\Delta T_{50}$, como el período de tiempo transcurrido desde el redshift al cual el primer evento de formación estelar tuvo lugar en la galaxia, hasta el

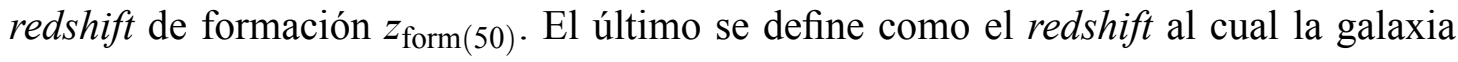
adquiere el 50 por ciento de su masa estelar total formada in-situ, donde el término insitu se refiere a la masa estelar formada en una galaxia por formación estelar quiescent (que ocurre en el disco) o en brotes estelares, sin tener en cuenta las estrellas acretadas de satélites. Esta definición asegura que las galaxias analizadas y sus poblaciones estelares son formadas de gas enriquecido perteneciente a sus progenitores principales, tornando más fácil la interpretación de la influencia de las escalas de tiempo y la eyección de productos de $\mathrm{SNs}$ en la relación $[\alpha / \mathrm{Fe}]$-masa estelar. Notar que la masa estelar total formada in-situ considerada para el presente análisis es rastreada en una variable separada dentro del código y no tiene en cuenta el efecto de pérdida de masa debido al proceso de reciclado de masa estelar que tiene lugar a lo largo y al final de la vida de las estrellas.

Fig. 4.11 muestra la escala de tiempo de formación, $\Delta T_{50}$, como función del redshift de formación, $z_{\text {form(50) }}$, para nuestra muestra entera de galaxias de tipo temprano obtenida de modelos con dos IMFs diferentes. Consideramos los modelos SAGTH5B2 (panel superior) y SAGS (panel inferior) que involucran una TH-IGIMF con $M_{\mathrm{ecl}}^{\mathrm{min}}=5 \mathrm{M}_{\odot}$ y $\beta=2$ y una IMF de Salpeter, respectivamente. La primera es nuestro mejor caso seleccionado de TH-IGIMF, como discutimos en la sección previa. Podemos ver que, a pesar de la IMF considerada, hay una clara tendencia en la cual $\Delta T_{50}$ decrece a medida que $z_{\text {form(50) }}$ crece, como muestra el gráfico de densidad en colores. Esto indica que las galaxias que se forman a más alto redshift completan el proceso de formación en escalas de tiempo más cortas, consistentemente con los resultados mostrados por Lagos, Padilla \& Cora (2009). Más específicamente, las galaxias que fueron formadas a $z_{\text {form(50) }} \approx 2$ 
o antes necesitaron un tiempo menor a 2 Gyr para alcanzar el 50 por ciento de su masa estelar formada in-situ.

A redshifts más bajos, la dispersión en las escalas de tiempo de formación se vuelve mayor. Los histogramas arriba y hacia la derecha del panel central, en los dos gráficos, representan las distribuciones de frecuencias del redshift de formación y las escalas de tiempo de formación, respectivamente. Vemos que la mayoría de las galaxias de tipo temprano se forman entre $z_{\text {form }(50)} \approx 1$ y 0 y en escalas de tiempo dentro de un intervalo $1 \mathrm{Gyr} \lesssim \Delta T_{50} \lesssim 4 \mathrm{Gyr}$.

Si consideramos la población global de galaxias formadas a diferentes redshifts, encontramos que la mayor parte de las galaxias tienen escalas de tiempo de formación menores que $2 \mathrm{Gyr}$, como es evidente de los histogramas hacia la derecha. Los valores medios y las medianas de las distribuciones de $\Delta T_{50}$ (líneas punteadas y a rayas en la figura, respectivamente) son 2,32 Gyr y 1,94 Gyr para el modelo SAGTH5B2, y 2,51 Gyr y 2,02 Gyr para el modelo SAGS, respectivamente.

Las distribuciones de $z_{\text {form(50) }}$ para galaxias de diferente masa son mostradas en la Fig. 4.12 para los modelos SAGTH5B2 y SAGS (paneles de arriba y abajo, respectivamente). De acuerdo con un trabajo previo de Lagos et al. (2009), ambos modelos presentan similares formas de sus distribuciones de $z_{\text {form }(50)}$ para diferentes intervalos de masa. Podemos ver que las galaxias menos masivas $\left(M_{\star}<10^{9} \mathrm{M}_{\odot}\right)$ logran el 50 por ciento de su masa estelar formada in-situ a redshifts más bajos que las galaxias más masivas, con la mayoría de ellas formándose entre $z \approx 0$ and $z \approx 0,5$. La tendencia de downsizing es menos pronunciada para galaxias más masivas que $\approx 10^{9} \mathrm{M}_{\odot}$. Los valores medios y medianas de las distribuciones (denotados por líneas punteadas y a rayas, respectivamente) son bastante similares para galaxias con masas dentre de los intervalos $10^{9}-10^{10} \mathrm{M}_{\odot}$ y $10^{10}-10^{11} \mathrm{M}_{\odot}, \mathrm{y}$ mayores que $10^{11} \mathrm{M}_{\odot}$, con valores abarcados dentro del intervalo $1 \lesssim z_{\text {form }(50)} \lesssim 1,5$. Sin embargo, el pico de las correspondientes distribuciones se corre del intervalo $0 \lesssim z_{\text {form(50) }} \lesssim 0,5$ a $0,5 \lesssim z_{\text {form(50) }} \lesssim 1$, haciendo evidente una tendencia de downsizing leve. El corrimiento del pico de las distribuciones es más evidente en el modelo SAGS (panel inferior).

Estos resultados están en acuerdo cualitativo con Lagos et al. (2009) y De Lucia et al. (2006). En este último trabajo, se muestra que las galaxias más masivas forman sus estrellas de manera más temprana que las menos masivas, pero que a su vez, ensamblan sus estrellas más tarde. Ellos definen el redshift de formación como el redshift cuando 

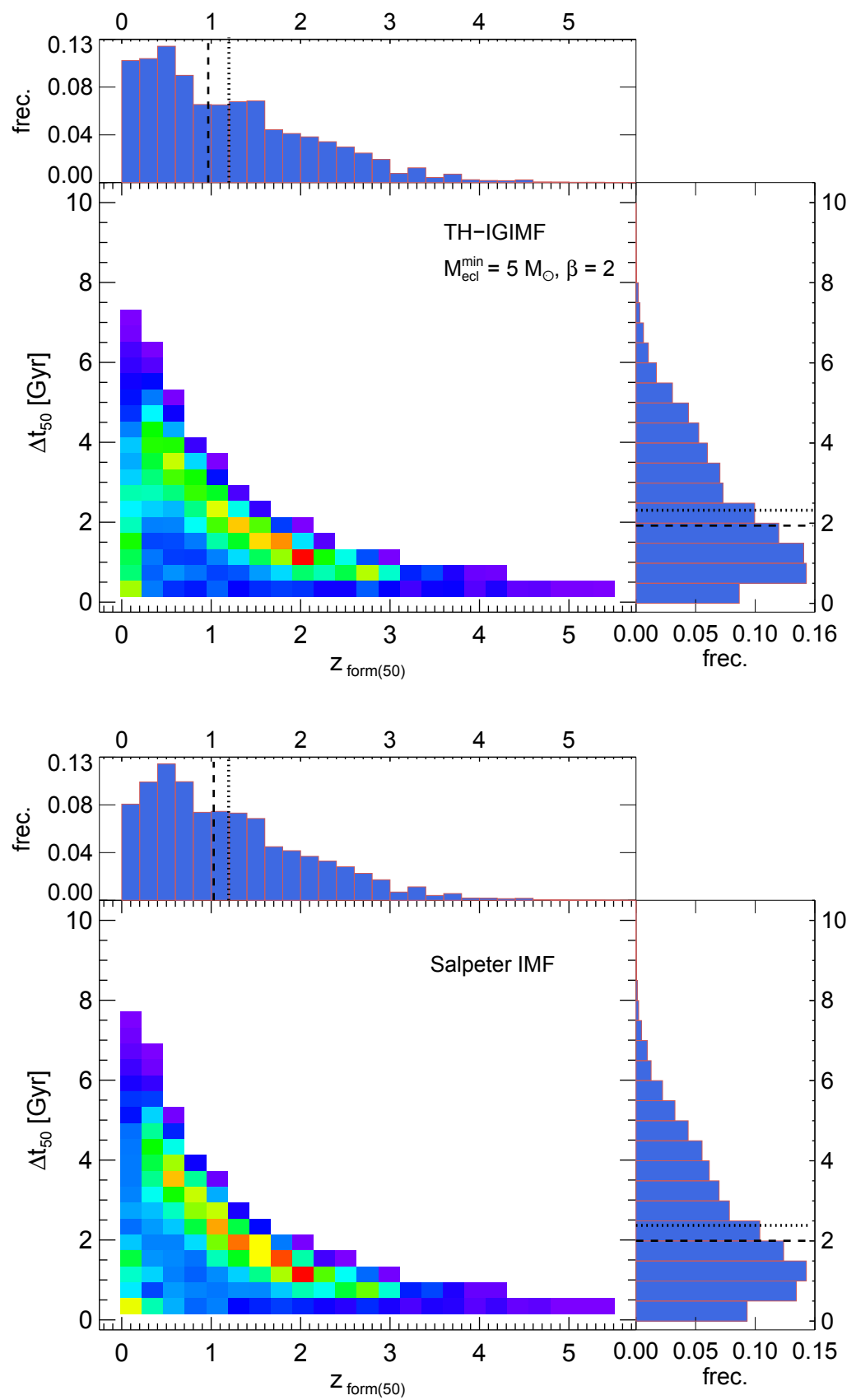

Figura 4.11: Escalas de tiempo de formación, $\Delta T_{50}$, de galaxias elípticas de los modelos versus sus respectivos redshifts de formación, $z_{\text {form(50) }}$ (ver el texto para su definición) para galaxias del modelo SAGTH5B2 (panel superior) y SAGS (panel inferior) Los histogramas a la derecha de los paneles centrales son las distribuciones de frecuencias de las escalas de tiempo de formación de la población de galaxias, y aquéllas mostradas por encima de los paneles centrales corresponden a los redshifts de formación. Líneas punteadas y a rayas representan la media y mediana de las distribuciones, respectivamente. 

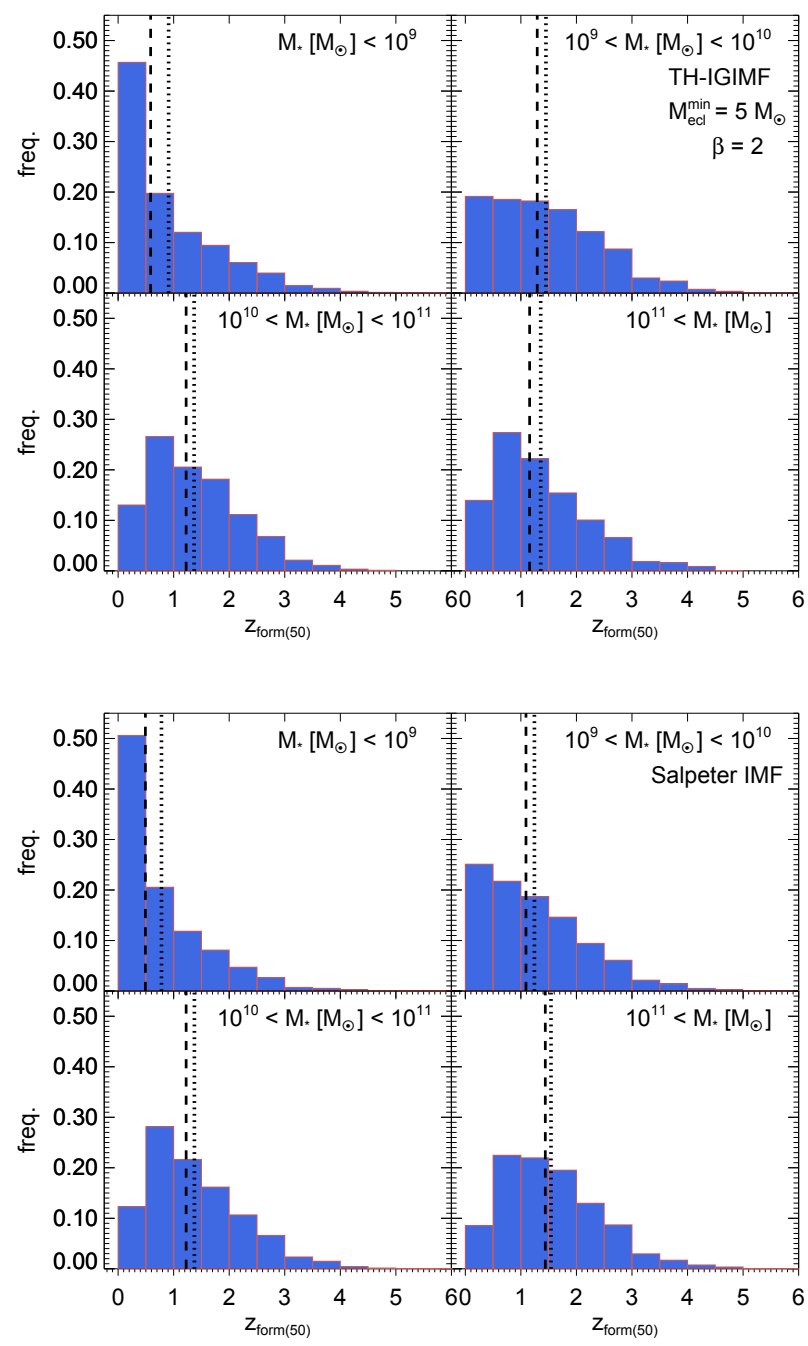

Figura 4.12: Distribución de redshifts de formación, $z_{\text {form(50) }}$, para galaxias de diferente masa estelar, como está indicado en las referencias del gráfico para los modelos SAGTH5B2 (panel superior) y SAGS (panel inferior). Líneas punteadas y a rayas representan la media y la mediana de las distribuciones, respectivamente. 
el 50 por ciento de las estrellas que forman la galaxia a $z=0$ ya están formadas, y el tiempo de ensamblado como el redshift cuando el 50 por ciento de la masa estelar final está contenida en un objeto solo. En nuestra definición de tiempo de formación no estamos considerando la contribución de la masa estelar formada en satélites acretados. Hemos evaluado el impacto de esta masa acretada en los histogramas mostrados en la Fig. 4.12, y no encontramos ningún cambio significativo, ni para el modelo SAGTH5B2 ni tampoco para SAGS. Ésto es esperable teniendo en cuenta que la masa contribuida por satélites es una fracción pequeña de la masa total final de la galaxia, siendo del orden de $\approx 10$ por ciento para la vasta mayoría de galaxias, de acuerdo con el análisis hecho por Jiménez et al. (2011).

Ahora nos concentramos en las distribuciones de $\Delta \mathrm{T}_{50}$ considerando galaxias en diferentes intervalos de masa. Esto es mostrado en la Fig. 4.13 para los modelos SAGTH5B2 y SAGS (paneles superior e inferior, respectivamente). Como en las distribuciones para la población entera de galaxias (Fig. 4.11), los valores de la media y la mediana (líneas punteada y a rayas, respectivamente) están muy cercanas una de la otra. El principal aspecto a destacar es que son muy similares a los valores correspondientes de la muestra completa de galaxias $(\approx 2-2,5 \mathrm{Gyr})$ y no presentan una gran dependencia con la masa estelar. Sólo las galaxias más masivas $\left(M_{\star}>10^{11}\right)$ tienen apenas mayores valores de media y mediana de $\Delta \mathrm{T}_{50}$. Esta situación es bastante similar para ambos modelos, indicando que las escalas de tiempo de formación de galaxias no están fuertemente afectadas por la elección de la IMF. Para poner en contexto estas escalas de tiempo de formación galáctica, realizamos una comparación con las escalas de tiempo necesarias para reproducir la correcta relación $[\alpha / \mathrm{Fe}]$-masa en el modelo de Pipino \& Matteucci (2004). Sus modelos reproducen pendientes correctas de $[\alpha / \mathrm{Fe}]$ como función de la masa de las galaxias suponiendo una caída de gas temprana con escalas de tiempo del orden de 0.4 Gyr y vientos galácticos que duren no más de $0.7 \mathrm{Gyr}$, dentro del paradigma de colapso monolítico, lo cual explica las diferencias con nuestras escalas de tiempo de formación galáctica.

La dependencia leve de las escalas de tiempo de formación con la masa estelar que emerge de nuestros modelos, con una IMF de Salpeter o una TH-IGIMF, muestran una tendencia opuesta a aquélla encontrada por De Lucia et al. (2006). Basados en el análisis de las historias de formación estelar de galaxias obtenidas de un modelo semianalítico, ellos muestran que las galaxias elípticas más masivas tienen escalas de tiempo de for- 

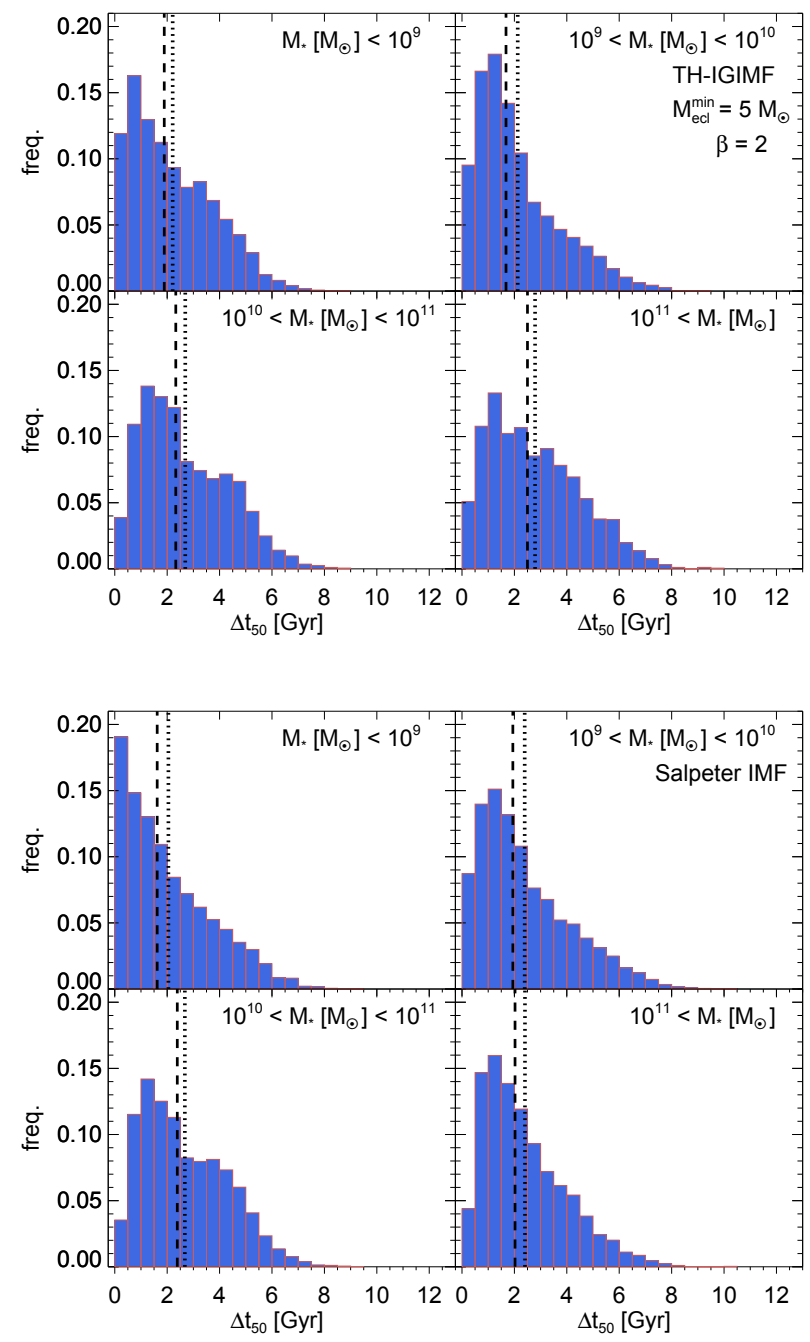

Figura 4.13: Distribución de escalas de tiempo de formación, $\Delta T_{50}$, para galaxias de diferente masa estelar, como está indicado en la referencia del gráfico, de los modelos SAGTH5B2 (panel superior) y SAGS (panel inferior). Las líneas punteadas y a rayas representan la media y mediana de las distribuciones, respectivamente 
mación más cortas que las menos masivas, en acuerdo cualitativo con el escenario de downsizing (Cowie et al., 1996) y con la dependencia masa estelar-escala de tiempo requerida por Thomas et al. (2005) para explicar la relación observada $[\alpha / \mathrm{Fe}]-$ masa estelar. Las diferentes tendencias obtenidas de nuestros modelos y de aquéllos de De Lucia et al. (2006) no son explicadas por el hecho de que no estamos considerando la formación estelar que tiene lugar en satélites acretados en nuestra definición de escala de tiempo de formación; como en las distribuciones de redshift de formación, evaluamos la influencia de la masa estelar acretada en satélites, sin encontrar un cambio significativo. Es de notar, sin embargo, que las extensiones del intervalo de tiempo en el cual la formación estelar tiene lugar presenta pequeñas diferencias entre las galaxias en diferentes intervalos de masa, tanto en nuestra muestra de galaxias modeladas como en aquéllas de De Lucia et al. (2006).

Esta leve tendencia de downsizing que emerge de nuestro modelo, como se muestra

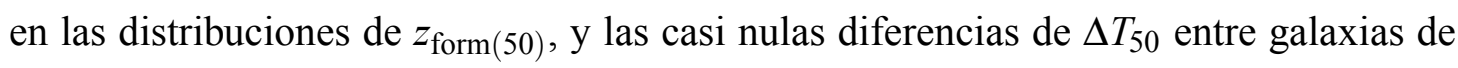
diferente masa, minimizan la relevancia de la explicación mas aceptada para interpretar los mayores cocientes de abundancias de $[\alpha / \mathrm{Fe}]$ en galaxias más masivas, lo cual es en general atribuido a su relativamente corta escala de tiempo de formación, como es inferido del análisis de datos observacionales (e.g. Thomas et al., 2005). Si esta diferencia en las escalas de tiempo de formación estuvieran presentes en las galaxias reales, contribuiría al desarrollo de una pendiente positiva en la relación, como es mostrado en otros modelos de formación galáctica (e.g. Pipino \& Matteucci, 2004). Sin embargo, esta explicación del downsizing quemo-archeológico puede ser descartada si tenemos en cuenta la falta de evidencia fuerte en términos de la evolución diferencial de la aglomeración de masa estelar o de evolución diferencial de la densidad de SFR de galaxias de diferentes masas, como surge del análisis de varios conjuntos de datos (Fontanot et al., 2009; Sobral et al., 2014). Nuestros modelos contemplan la posibilidad de que galaxias masivas alcancen mayores cocientes de abundancia de $[\alpha / \mathrm{Fe}]$ porque están caracterizados por IMFs más planas. Nuestro estudio refuerza esta idea por la similitud de las distribuciones de $z_{\text {form(50) }}$ y $\Delta \mathrm{T}_{50}$ y por la relación entre ellas para modelos con una IMF de Salpeter y una TH-IGIMF, indicando que las escalas de tiempo de formación de las galaxias no juegan un rol decisivo en determinar la pendiente de la relación $[\alpha / \mathrm{Fe}]-$ masa estelar seguida por las galaxias generadas por estos dos modelos. El resultado más relevante de nuestro trabajo es que una TH-IGIMF es necesaria para reproducir la ten- 
dencia observada. Tenemos que tener presente que aunque nuestro modelo presenta una tendencia de downsising leve en términos del redshift característico de formación de la galaxia y escalas de tiempo de formación, la última es la cantidad que importa en la interpretación de la relación $[\alpha / \mathrm{Fe}]$-masa estelar. Sin embargo, la principal evidencia observacional del downsising en términos de estas escalas de tiempo es inferida de la relación misma.

A partir de este importante resultado, consideramos galaxias del modelo SAGTH5B2 y analizamos la conexión entre la distribución de cocientes de abundancia $[\alpha / \mathrm{Fe}] \mathrm{y}$ las escalas de tiempo de formación $\Delta \mathrm{T}_{50}$ para comprender el origen de las principales características de las relaciones $[\alpha / \mathrm{Fe}]$-masa estelar de galaxias con una TH-IGIMF caracterizada por $M_{\mathrm{ecl}}^{\min }=5 \mathrm{M}_{\odot}$. Esto se muestra en la Fig. 4.14, donde los diferentes paneles corresponden a galaxias en diferentes intervalos de masa. En todos los casos, podemos ver que los valores más grandes de cocientes de abundancia de $[\alpha / \mathrm{Fe}]$ son logrados para escalas de tiempo de formación más cortas. El aspecto importante para remarcar es que el argumento de escalas de tiempo nos permite explicar la dispersión de valores de $[\alpha / \mathrm{Fe}]$ para galaxias de una dada masa, pero no es la razón del desarrollo de una pendiente en la relación $[\alpha / \mathrm{Fe}]$-masa estelar. Esta dependencia entre los cocientes de abundancia y la masa estelar es sólo posible lograrla en nuestro modelo considerando una IMF dependiente de la SFR como la TH-IGIMF probada aquí.

Relacionando el resultado mostrado en la Fig. 4.14, el cual indica que las galaxias con escalas de tiempo de formación más cortas tienen valores más grandes de $[\alpha / \mathrm{Fe}]$, con lo que se aprecia de la Fig. 4.11, donde resulta claro que las galaxias más viejas (aquéllas con más alto $z_{\text {form(50) }}$ ) son formadas en tiempos más cortos, concluimos que las galaxias más viejas logran mayores valores de $[\alpha / \mathrm{Fe}]$. Esto es consistente con los datos observacionales analizados por Sánchez-Blázquez et al. (2006), que encuentran que los modelos que mejor reproducen las pendientes observadas son aquéllos en los cuales los elementos $\alpha$ varían más que los elementos del pico del Fe a lo largo de la secuencia de dispersión de velocidades; además, galaxias más viejas a una dada dispersión de velocidades muestran mayores cocientes de abundancias $[\alpha / \mathrm{Fe}]$. Estos resultados apoyan descubrimientos previos de Trager et al. (2000), quienes muestran que elípticas más jóvenes tienen mayores abundancias $[\mathrm{Fe} / \mathrm{H}]$ que las más viejas. 


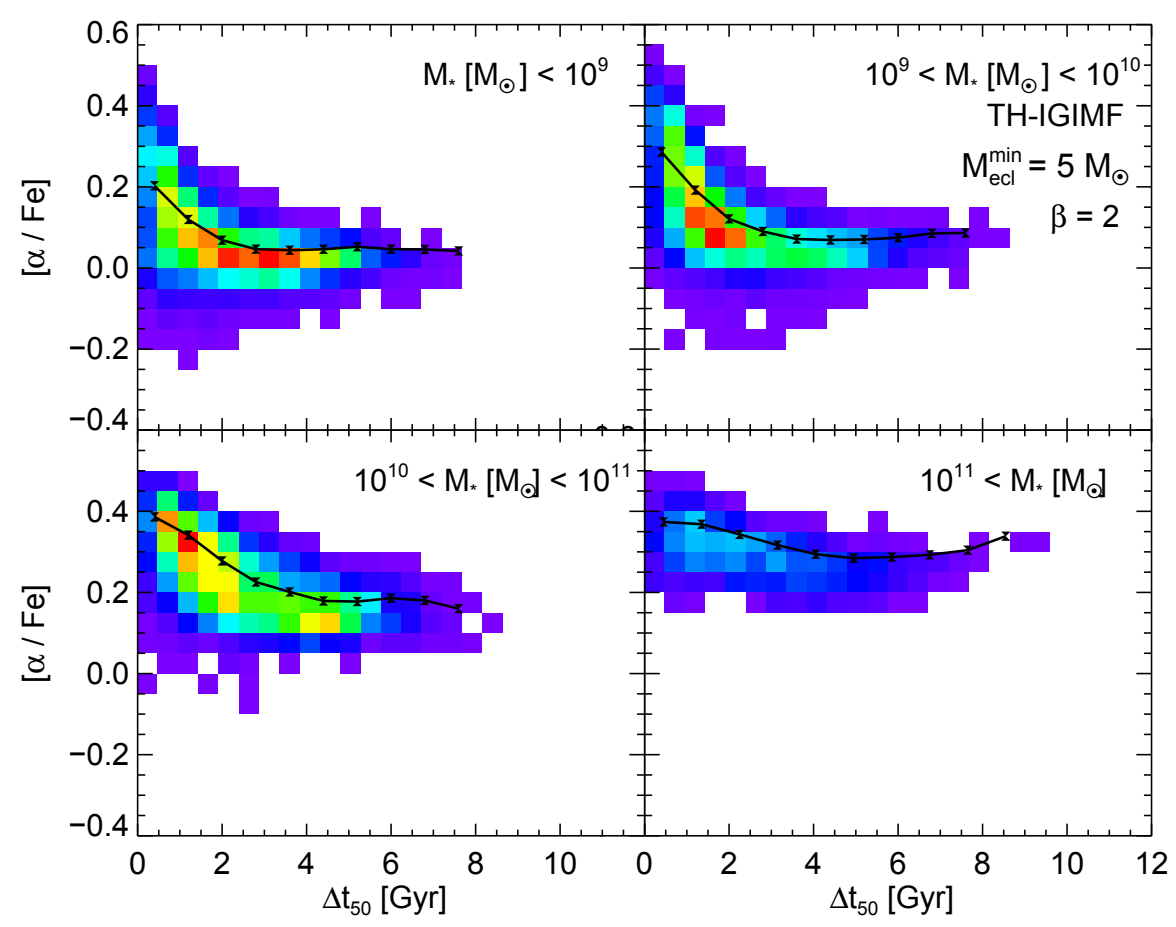

Figura 4.14: Distribuciones de cocientes de abundancia de $[\alpha / \mathrm{Fe}]$ como función de la escala de tiempo de formación, $\Delta \mathrm{T}_{50}$, para galaxias dentro de diferentes intervalos de masa, como está indicado en las referencias del gráfico, para el modelo SAGTH5B2, caracterizado por $M_{\mathrm{ecl}}^{\min }=5 \mathrm{M}_{\odot}$ y $\beta=2$. La línea sólida en cada panel conecta los valores medios de los cocientes de abundancia para diferentes bines de $\Delta \mathrm{T}_{50}$.

\section{Discusión}

La tendencia de $[\alpha / \mathrm{Fe}]$ con la masa estelar en galaxias de tipo temprano ha sido estudiada en el pasado, tanto observacionalmente como teóricamente. Sin importar los métodos observacionales, existe un acuerdo en que las abundancias relativas de elementos livianos (producidos casi exclusivamente por SNs II) con respecto al hierro (producido principalmente por SNs Ia) se incrementa monotónicamente con la dispersión de velocidades central (por lo tanto, masa de la galaxia). En el lado teórico, una considerable cantidad de trabajo ha sido dedicado a estudiar este aspecto de evolución química dentro del marco de modelo cosmológico $\Lambda \mathrm{CDM}$, principalmente porque ha sido señalado como un problema del modelo, mostrando tensión entre el esperado crecimiento jerárquico 
de estructura, de menor a mayor, y el comportamiento de downsizing de las galaxias.

Dos principales hipótesis han sido propuestas para explicar la tendencia observada, las escalas de tiempo de formación de las galaxias y una IMF variable. De acuerdo al argumento de las escalas de tiempo, se supone que las poblaciones estelares en galaxias con mayores masas estelares se forman en escalas de tiempo más cortas, evitando el enriquecimiento de sus medios interestelares por SNs Ia que son caracterizadas por tiempos de retardo de explosión largos. En el caso de la hipótesis de una IMF variable, el cociente variable entre estrellas de alta masa y baja masa en la distribución inicial de estrellas en diferentes galaxias se supone que juega un rol crucial en la construcción del patrón de abundancias observado en galaxias de tipo temprano, dado que este cociente determina el cociente entre SNs II y SNs Ia.

En los últimos años, han habido varios estudios de galaxias de tipo temprano y masivas sugiriendo que sus poblaciones estelares pueden haber sido formadas con diferentes IMFs que aquéllas estimadas a partir del vecindario solar. Hay indicaciones de que la luz de estrellas en galaxias masivas de tipo temprano es consistente con poblaciones formadas con una IMF bottom-heavy (con dominio de estrellas de baja masa), determinado por el análisis de sus rasgos espectrales (van Dokkum \& Conroy, 2010, 2012; Ferreras et al., 2013; La Barbera et al., 2013; Conroy et al., 2013). En otros trabajos, el cociente masa-luminosidad de galaxias masivas de tipo temprano es restringido usando herramientas dinámicas (Cappellari et al., 2012; Dutton et al., 2012; Tortora et al., 2013) o lentes gravitacionales fuertes (Auger et al., 2010; Treu et al., 2010).

Sin embargo, estos métodos presentan una degeneración en el sentido de que un cociente de estrellas poco masivas mayor implica tanto la existencia de una IMF bottom heavy o una IMF top heavy, ya que las estrellas masivas terminan su vida en escalas de tiempo cortas y contribuyen a la luz total emitida sólo durante eventos de formación estelar y poco tiempo después. Weidner et al. (2013) estudian el problema con un toy model que propone una IMF top heavy a alto redshift durante condiciones de brotes estelares tempranos y una IMF bottom heavy en la evolución posterior. En su modelo, el régimen de alta formación estelar conduce a la fragmentación del ISM favoreciendo la formación de estrellas de baja masa. Un aspecto importante que ellos remarcan es que la IMF bottom heavy propuesta por varios trabajos, como Ferreras et al. (2013), puede subestimar severamente la cantidad de metales presente en galaxias elípticas masivas, dado que la mayor parte de los metales son eyectados al ISM por estrellas con masas 
sobre $1 \mathrm{M}_{\odot}$.

En general, cuando el argumento de una IMF variable es considerado para explicar la relación $[\alpha / \mathrm{Fe}]$-masa estelar, la IMF propuesta es tratada como un parámetro libre (Arrigoni et al., 2010), o es modificada con fines exploratorios, (Thomas, 1999; Nagashima et al., 2005; Calura \& Menci, 2009), sin seguir ninguna teoría particular y dejando inexplorado una vasta región del correspondiente espacio de parámetros. En este trabajo, nosotros probamos la teoría bien definida que tiene en cuenta la función inicial de masa integrada de estrellas en galaxias con IMFs top heavy en cúmulos estelares durante brotes estelares (ver Sección 8.2 del Capítulo 3 para una descripción breve y referencias a los trabajos originales). Hemos mostrado que el cociente de abundancias de $[\alpha / \mathrm{Fe}]$ es bien reproducido suponiendo una TH-IGIMF dependiente de la SFR; nuestros resultados apoyan un valor de $5 \mathrm{M}_{\odot}$ para la masa mínima de cúmulo inmerso y $\beta=2$ para la pendiente de la función de masa de cúmulos inmersos, los cuales son parámetros libres de su teoría. Recchi et al. (2009, R09 de aquí en más) ya habían considerado la teoría de IGIMF para evaluar el impacto en el desarrollo de la relación $[\alpha / \mathrm{Fe}]-\sigma$ y explorar diferentes valores para la pendiente de la función de masa de cúmulos embebidos ( $\beta$ 1,2 and 2,35). Ellos hacen uso de una versión previa de la IGIMF, en la cual el efecto de altos valores de formación estelar en galaxias no era considerado. Cuando estos altos valores de SF son abarcados, la IMF dentro de cúmulos se puede volver top heavy debido a efectos de crowding o sobrepoblación. En tales condiciones, la SFR cambia con la consecuente reducción en los valores de las pendientes de la IGIMF, en la versión THIGIMF. Hay importantes diferencias entre los modelos usados en R09 y aquéllos usados en este trabajo. Nosotros utilizamos un modelo semianalítico con árboles de fusión que considera la evolución de las galaxias elípticas en el marco de un universo $\Lambda$-CDM. El modelo analítico usado en R09 considera la evolución de las galaxias elípticas desde el punto de vista del colapso monolítico. Otro aspecto fundamental es el tratamiento de la retroalimentación de SN y AGN. Nuestro modelo tiene en cuenta la retroalimentación producida por las estrellas consistentemente con la elección de la pendiente de la TH-IGIMF. Esto significa que valores más bajos de las pendientes de la TH-IGIMF conducen a numeros más altos de SNs II que recalientan el gas, regulando la formación de nuevas generaciones de estrellas y poniendo restricciones fuertes a los parámetros libres de la teoría de la TH-IGIMF. Por ejemplo, para $M_{\text {ecl }}^{\min }=100 \mathrm{M}_{\odot}$ o hasta para la pendiente de la función de masa de los cúmulos embebidos $\beta=2,1$, encontramos 
difícil calibrar el modelo dados los altos niveles de retroalimentación que sufren. La retroalimentación de AGNs en galaxias, el cual no es considerado en los modelos de R09, es también un aspecto importante porque previene que las galaxias masivas continúen formando estrellas a bajos redshifts. Esto contribuye a determinar la leve tendencia de downsizing vista en nuestro modelo, aunque se esperaría un efecto más fuerte.

Un problema asociado a las abundancias de elementos $\alpha$ en galaxias de tipo temprano está relacionado a las abundancias de calcio $(\mathrm{Ca})$. Comúnmente, se espera que todos los elementos ligeros producidos por captura- $\alpha$ se tracen entre sí como resultado de su origen común. Sin embargo, la abundancia de Ca respecto al hierro no sigue a la abundancia del magnesio (Mg) o el titanio (Ti) relativa al hierro como función de la dispersión central de velocidades o masa estelar en galaxias de tipo temprano. Mientras los índices espectrales sensibles al Mg o al Ti muestran un incremento de sus abundancias con el incremento de la masa, algunos índices sensibles al Ca muestran una disminución de la abundancia de este elemento con la masa (Saglia et al., 2002; Cenarro et al., 2003, 2004). Recientemente, Worthey et al. (2013) confirman esta tendencia engañosa con nuevas observaciones y evalúan diferentes hipótesis para explicarlo. Ellos encuentran que una mayor pendiente en la IMF a masas de estrellas bajas es descartada por las restricciones en colores de las galaxias. Es interesante notar que una IMF favoreciendo la formación de estrellas masivas está en acuerdo con sus resultados, siempre y cuando los yields de Ca dependan de la masa del progenitor de SN II. Los yields de Woosley \& Weaver (1995) muestran una disminución del Ca con respecto al hierro a medida que se incrementa la masa de la estrella progenitora. Sin embargo, Nomoto et al. (2006) and Kobayashi et al. (2006) muestran que no hay dependencia del yield de Ca con la masa del progenitor. Si esta tendencia de los yields de SNs II para el Ca es real, una IMF top-heavy, como la probada en este trabajo sería necesaria para explicar la correlación del calcio con la masa de galaxias de tipo temprano, ya que la formación de estrellas masivas sería favorecida durante la formación de galaxias más masivas.

Un trabajo reciente de Conroy et al. (2013) muestra más tendencias interesantes entre otros elementos individuales. Ellos estudian los patrones de abundancias del bario (Ba) y el estroncio ( $\mathrm{Sr}$ ) y encuentran que el cociente de abundancias relativas $[\mathrm{Ba} / \mathrm{Mg}]$ muestra un decrecimiento respecto al cociente $[\mathrm{Mg} / \mathrm{Fe}] \mathrm{y}$, aunque no lo muestran en su trabajo, declaran una fuerte correlación entre $[\mathrm{Mg} / \mathrm{Ba}]$ y la dispersión de velocidades en una muestra de galaxias de tipo temprano extraídas del SDSS. La importancia de 
este descubrimiento radica en el hecho de que el $\mathrm{Ba}$ es un elemento que es producido por procesos-s en estrellas de masa baja e intermedia durante la fase de la rama gigante asintótica (AGB) de su evolución. Así, el cociente de abundancias $[\mathrm{Mg} / \mathrm{Ba}]$ puede ser usado como prueba de escalas de tiempo de formación cortas como también como indicador de cociente de estrellas de alta masa y baja masa, de igual manera que el cociente de abundancias $[\mathrm{Mg} / \mathrm{Fe}]$. Pero esto es más que una medida alternativa, porque permite dejar de lado la influencia de las SNIa. Consecuentemente, si es real, este nuevo patrón de abundancias permitirá descartar algunas explicaciones para la relación $[\alpha / \mathrm{Fe}]$-masa estelar en galaxias de tipo temprano que involucran diferentes distribuciones de tiempos de retardo para las SNs Ia (e.g. Yates et al., 2013). 


\section{Capítulo 5}

\section{Poblaciones estelares del bulbo de galaxias tipo Vía Láctea ${ }^{1}$}

En el presente capítulo se desarrolla un trabajo de investigación orientado a estudiar las propiedades químicas de las poblaciones estelares (PEs) de bulbos de galaxias tipo Vía Láctea (VL). El objetivo general es contribuir al entendimiento de los mecanismos de formación de los bulbos y de la historia de formación del bulbo de la VL en particular.

\section{Introducción}

El problema de la formación del bulbo de la VL permanece vigente. Hoy es bien sabido que las fusiones y las interacciones de galaxias juegan un rol importante en la formación de los bulbos (Kormendy \& Kennicutt, 2004). Esto ocurre predominantemente a altos redshifts debido al hecho de que en un universo $\Lambda \mathrm{CDM}$, el número relativo de fusiones es mayor a tiempos más tempranos, mientras que a redshifts más bajos, la tasa de fusiones decrece y las galaxias evolucionan secularmente (Athanassoula, 2013); las escalas de tiempo de formación son más largas que el tiempo de colapso.

Durante la evolución secular, los discos galácticos con altas densidades superficiales pueden volverse inestables frente a pequeñas perturbaciones (Mo et al., 1998) o fusiones

\footnotetext{
${ }^{1}$ El presente capítulo está basado en un trabajo en preparación: Resolving stellar populations in a SAM: bulges of MW type galaxies. Autores: Gargiulo, I. D, Cora, S. A., Padilla, N. D., González, O., Zoccali, M., González, R.
} 
(Padilla et al., 2013), produciendo una barra que transfiere gas y estrellas del disco al bulbo. A partir de la disipación del gas se forman estrellas en brotes (Kormendy, 2013).

En este complejo contexto, el caso del bulbo de la VL representa nuestra mejor oportunidad para traer algo de luz sobre el problema de la formación de los bulbos. En los últimos años, han habido esfuerzos para observar el bulbo en el infrarojo cercano, como es el caso del relevamiento de Variables Vista en la Vía Láctea (VVV Minniti \& et al., 2010), donde los efectos de extinción son mucho menores que en longitudes de onda ópticas. Ahora podemos observar estrellas individualmente y tener acceso a toda la información que contienen en sus propiedades. Aunque la mayor parte de la luz en infrarrojo es aportada por gigantes rojas, ha sido posible observar estrellas en el turnoff de digramas color-magnitud (CMD, por sus siglas en inglés, Bensby et al., 2011, 2013) del bulbo con técnicas de micro-lente gravitacionales. Un relevamiento anterior en el infrarrojo de todo el cielo como 2MASS (Two Micron All-Sky Survey, Skrutskie et al., 2006) permitió establecer la forma de maní del bulbo de la VL (López-Corredoira et al., 2005). La existencia de una división en la nube de gigantes rojas metálicas en latitudes altas del bulbo parece confirmar que el bulbo presenta una forma de $\mathrm{X}$ consistente con un bulbo tipo boxy/maní (McWilliam \& Zoccali, 2010). Estos tipos de bulbo se caracterizan por ser dinámicamente más fríos que los bulbos generados por fusiones, dinámicamente calientes y conocidos como bulbos clásicos, que fueron descriptos como "elliptical galaxies that happen to have a disc around them"2(Gadotti, 2009). Sin embargo, es muy probable que las fusiones sufridas por la VL en el pasado hayan contribuido a la formación de su bulbo, y se ha afirmado que existen poblaciones estelares que tienen diferentes orígenes. Algunos trabajos aseveran que coexisten dos poblaciones estelares diferenciadas en edad, metalicidad, escalas temporales de formación o enriquecimiento de elementos $\alpha$ y cinemática (Feltzing \& Gilmore, 2000; Groenewegen \& Blommaert, 2005; Zoccali et al., 2008; Soto et al., 2007, e.g.). Trabajos más recientes van más lejos y afirman que el bulbo está compuesto por poblaciones estelares múltiples originadas en diferentes eventos de inestabilidad del disco (Ness et al., 2012, 2013). Aunque las determinaciones de edad de las estrellas del bulbo de la VL utilizando CMDs dan como resultado que la mayoría son más viejas que $10 \mathrm{Gyr}$ y apoyan la idea de una única población estelar (Zoccali et al., 2003), las observaciones de Bensby et al. $(2011,2013)$ de estrellas enanas con micro-lentes dan muestra de que la edad de estas estrellas ocupan

\footnotetext{
${ }^{2}$ Galaxias elípticas que ocurre que tienen un disco a su alrededor
} 
un intervalo entre 2 y 13 Gyr. Las simulaciones de formación de bulbos pueden explicar la existencia de estrellas jóvenes bajo la hipótesis de formación por inestabilidad gravitacional del disco (Ness et al., 2014).

Las PEs contienen una vasta cantidad de información sobre la formación y evolución de los sistemas estelares. En particular, las abundancias químicas de las PEs son una herramienta fundamental para validar las hipótesis de formación de los bulbos.

En este trabajo, nuestro objetivo es ganar conocimiento sobre los mecanismos de formación de los bulbos haciendo un uso novedoso del modelo semianalítico de formación de galaxias SAG, alcanzando la escala de PEs de galaxias tipo VL, y comprender aspectos de las observaciones de estrellas existentes en el bulbo de la VL. La inclusión del seguimiento de las propiedades de las PEs individualizadas en un modelo semianalítico de formación de galaxias se presenta como una herramienta novedosa y muy útil para estudiar las características de la formación de las componentes de las galaxias como el bulbo y el halo estelar. Esta nueva capacidad permite estudiar, por ejemplo, desde un punto de vista teórico, cómo se distribuyen las PEs de distintos orígenes en el espacio de las abundancias químicas, ayudando a interpretar las observaciones disponibles.

Así, el presente trabajo contribuye a dar un marco teórico a la técnica conocida como "etiquetado químico" de estrellas (del inglés chemical tagging). La idea del etiquetado químico de estrellas fue concebida por Freeman \& Bland-Hawthorn (2002) y consiste en medir las abundancias químicas de la mayor cantidad posible de estrellas de una dada componente de nuestra galaxia, como el disco, bulbo o halo, o bien de una galaxia satélite de nuestra Vía Láctea, para luego ubicarlas en el espacio de abundancias químicas. De esta manera, las estrellas que comparten un origen, como una agregación estelar particular, o un cúmulo disuelto, poblarán regiones cercanas en diagramas de abundancias químicas. Si tomamos, por ejemplo, el caso del bulbo de la Vía Láctea, un conjunto de estrellas que fueron acretadas de una dada galaxia en una fusión pasada, o que provienen del disco inestabilizado de algúna época en la historia de la galaxia, compartirán abundancias similares. Nuestro modelo permite transitar el camino inverso. Conociendo el origen de las PEs, podemos estudiar por qué ocupan el lugar que ocupan en un diagrama de abundancias.

En este capítulo analizamos las propiedades químicas de las PEs de bulbos de galaxias tipo VL. Una vez más, como en el caso de las galaxias elípticas, distintas suposiciones sobre la IMF adoptada durante la formación estelar del bulbo puede proveer 
interpretaciones alternativas sobre la historia de formación de esta componente. Aceptamos como válidas para este caso las suposiciones realizadas para aplicar una IMF integrada galáctica (ver Sección 8.2). Consideramos que el bulbo, al igual que una galaxia, está formado por múltiples eventos de formación estelar que forman cúmulos, muchos de ellos disueltos dinámicamente con posterioridad. Comparamos resultados utilizando la IMF de Salpeter y la mejor elección de TH-IGIMF encontrada en el Cap. 4, caracterizada por los parámetros $\beta=2$ y $M_{\mathrm{ecl}}^{\min }=5 \mathrm{M}_{\odot}$ correspondientes a la masa mínima de cúmulo estelar inmerso y a la pendiente de la función de masa de cúmulos inmersos, respectivamente. Nos referiremos a esta IMF simplemente como TH-IGIMF a lo largo del capítulo. Las calibraciones de estos dos modelos, SAGS y SAGTH5B2, se corresponden con aquéllas utilizadas en el Capítulo 4, las cuales reproducen de manera correcta el conjunto de observables presentado en la Sección 3 de ese capítulo y con los cuales se logra modelar las propiedades químicas de las galaxias elípticas. Este hecho cobra especial relevancia ya que los mecanismos de formación de las galaxias elípticas en el modelo son los mismos que los de los bulbos.

\section{Selección de galaxias tipo VL}

La selección de galaxias tipo VL fue realizada siguiendo dos criterios diferentes. Por un lado, utilizamos el criterio de selección definido por Mutch et al. (2011, M11 de aquí en adelante) en el que una galaxia es considerada como tipo VL si se cumplen 3 condiciones:

- Ser la galaxia más masiva en el halo de MO que la contiene, lo que se traduce en términos de la simulación a ser una galaxia central de un halo FOF, es decir, de tipo 0 (Ver Sección 1) en el Capítulo 3.

- Tener una masa estelar en el intervalo $10^{10,5} \leq M\left[M_{\odot}\right] \leq 10^{11,2}$. Esta condición resulta equivalente al que define a la muestra del galaxy Zoo de galaxias de canto tipo VL / M31 en el mismo trabajo de M11.

- Tener una morfología aproximada a una galaxia $\mathrm{Sb} / \mathrm{c}$. Esta condición se puede considerar cumplida si la diferencia entre la magnitud media del bulbo y la total 
está en el intervalo $1,5 \leq M_{\mathrm{B}-\text { bulbo }}-M_{\mathrm{B} \text {-total }} \leq 2,6$, siguiendo una definición de Simien \& de Vaucouleurs (1986).

La muestra final obtenida aplicando este criterio es de 398 galaxias tipo VL para el modelo con una IMF de Salpeter y de 2835 galaxias VL para el modelo con la THIGIMF, sobre un total de 812,409 y 812,228 galaxias, respectivamente.

El segundo criterio de identificación de galaxias tipo VL considera la pertenencia de galaxias a sistemas análogos al Grupo Local (LG, por su sigla en inglés). Los sistemas análogos al LG son identificados considerando varias restricciones, de acuerdo al trabajo de González et al. (2014, G14 de aquí en más):

- Primero, se buscan pares de halos host (del inglés de anfitrión), referido a los halos que contienen a las galaxias tipo VL o M31 en este caso, en los cuales ambos miembros tienen masas en un amplio intervalo de masas desde $M_{200 c}=$ $1 \times 10^{11} \mathrm{M}_{\odot}$ a $1 \times 10^{13} \mathrm{M}_{\odot}{ }^{3}$ y están separadas por $0,5-1,3 \mathrm{Mpc}$.

- Segundo, para seleccionar pares con cierto aislamiento y para evitar pares en tripletes o grupos más grandes, se define un criterio cuantitativo de aislamiento usando la restricción de fuerza $F_{i . c o m}<\kappa F_{12}$, donde $F_{i \text {,com }}$ es la fuerza gravitacional entre el par y cualquier halo vecino $i$ dentro de un radio de $5 h^{-1} \mathrm{Mpc}$ del centro de masa del par, $F_{12}$ es la fuerza entre el par, y $\kappa$ es un parámetro constante. El criterio de aislamiento se vuelve cada vez más estricto para valores decrecientes de $\kappa$. La VL y M31 no tienen halos vecinos y masivos cercanos dentro de $5 \mathrm{Mpc}$, y deben, por lo tanto, tener $\kappa<0,1$. El valor real de $\kappa$ es, sin embargo, incierto, y se usa un valor más relajado de $\kappa=0,25$ basados en pruebas previas reportadas en González et al. (2013).

- Con el tercer criterio de selección, se tiene la intención de emular la ausencia de cúmulos masivos en la vecindad inmediata del LG. Se requiere que los halos en la muestra de sistemas análogos al LG no tenga un halo vecino con masa

\footnotetext{
${ }^{3}$ Definida como la masa contenida dentro de un radio que encierra una densidad media de 200 veces la densidad crítica en el redshift en el cual se realiza el análisis. Para halos del tamaño correspondiente a la VL, esta cantidad está relacionada con la comúnmente usada definición de masa virial basada en la sobredensidad dependiente de la cosmología y del redshift como $M_{200 c} / M_{\mathrm{vir}} \approx 1,2$ (Bryan \& Norman, 1998).
} 
$M_{200 c}>1,5 \times 10^{14} \mathrm{M}_{\odot}$ dentro de $12 \mathrm{Mpc}$. Los límites de masa y de distancia son de alguna manera más bajos que los valores reales del cúmulo de Virgo.

- Cuarto, se imponen restricciones en la velocidad radial galactocéntrica, la velocidad tangencial y la separación de los pares, basados en la literatura, pero con errores amplificados a $V_{\mathrm{RAD}}=-109,3 \pm 80 \mathrm{~km} \mathrm{~s}^{-1}, V_{\mathrm{TAN}}<65 \mathrm{~km} \mathrm{~s}^{-1}$ y $\Delta r=770 \pm 100 \mathrm{kpc}$, respectivamente, para obtener una muestra más relajada.

Más detalles sobre la identificación de los sistemas análogos al LG y valores de referencia se pueden encontrar en el trabajo de G14.

De los 909 pares aislados encontrados usando los tres primeros criterios de referencia, sólo 19 pares sobreviven el cuarto criterio. Es importante notar que ambas galaxias de cada par son consideradas como de tipo VL, dando un total de 38 galaxias tipo VL para analizar, y que los halos seleccionados son los mismos en los modelos con la IMF de Salpeter y con la TH-IGIMF.

La selección de galaxias tipo VL considerando las condiciones de entorno del grupo local es más consistente con la historia de formación galáctica que sufrió la VL que el método de M11 y está menos sujeta a errores sistemáticos que pueden tener su origen en bias causados por incluir distintos tipos de ambientes. Sin embargo, el rango dinámico de la selección realizada utilizando el criterio de M11 hace que la muestra esté menos condicionada por errores estadísticos. Ambas presentan ventajas, de manera que consideramos los dos tipos de selecciones para estudiar las PEs en los bulbos.

En el panel superior de la Fig. 5.1 puede observarse la diferencia de magnitudes, o cociente de luminosidades, en el filtro B en función de la masa estelar de las galaxias del modelo SAGS que utiliza una IMF de Salpeter. Con líneas rojas se delimita la región de este espacio ocupado por las galaxias tipo VL según el criterio de M11; las mismas se señalan con círculos de color verde oscuro. Las galaxias seleccionadas con el criterio de G14 son identificadas con círculos de rojos. Como puede observarse, los dos conjuntos no presentan elementos en común, con excepción de una de las galaxias que resulta seleccionada por el criterio de G14 y se encuentra dentro del cuadrado delimitado por líneas rojas correspondiente al criterio de M11. Si nos concentramos en las galaxias contenidas en grupos tipo LG, muchas están en el intervalo adecuado de masas estelares galácticas definido por M11 e indicado con líneas verticales rojas en la figura, pero con valores de la diferencia de magnitud del bulbo y la magnitud total cercanos a cero, 

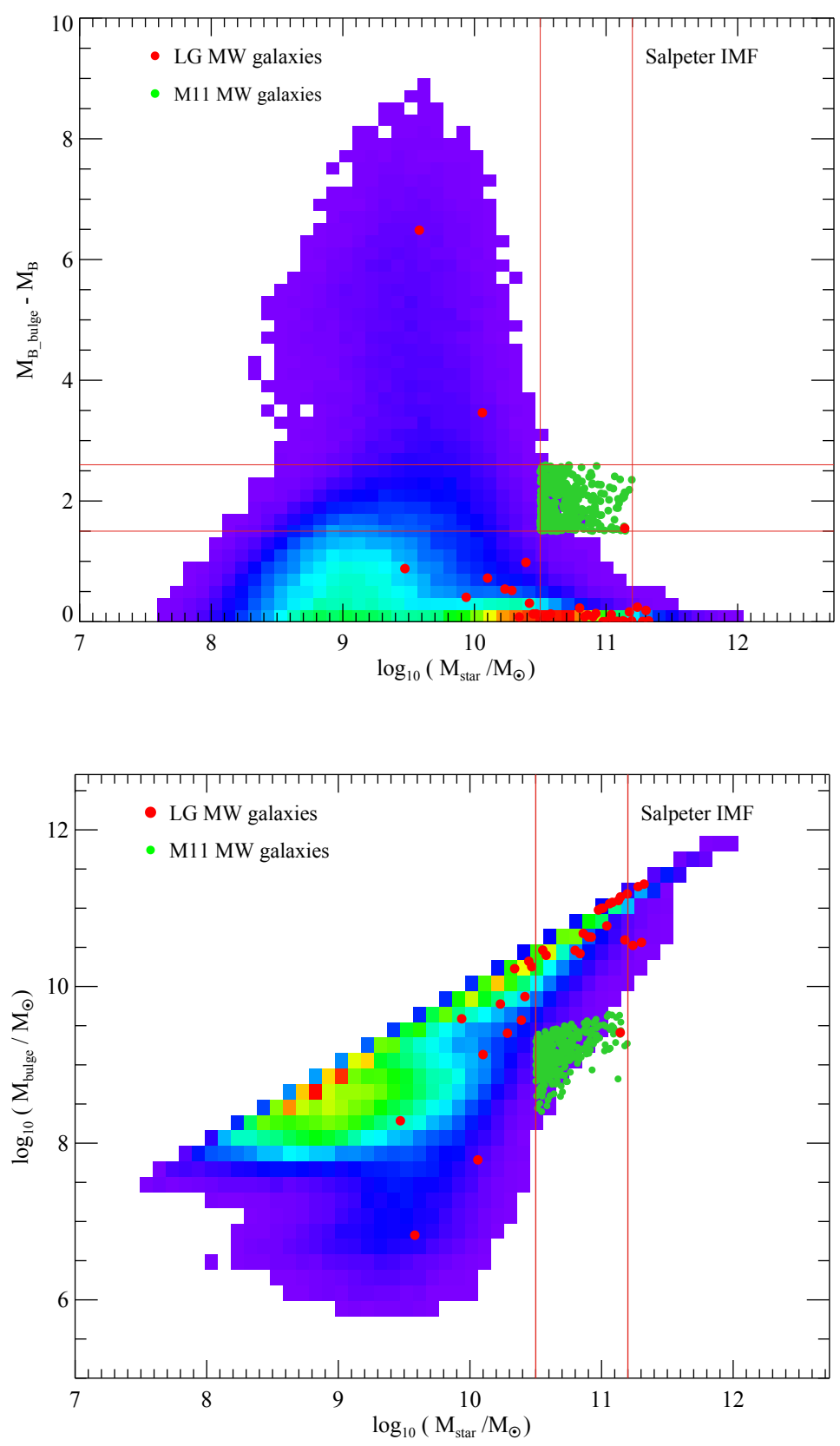

Figura 5.1: Panel superior: Diferencia de magnitudes (cociente de luminosidades) en el filtro B como función de las masas estelares de las galaxias del modelo SAGS utilizando una IMF de Salpeter. Se delimita con líneas rojas el intervalo de masas estelares definido en el criterio de M11. Con círculos verde oscuro se señalan las galaxias que cumplen con ese criterio. Con círculos rojos se señalan las galaxias tipo VL que respetan el criterio de grupos locales de G14. Panel inferior: Masa de bulbo en función de la masa estelar de las galaxias de la simulación utilizando una IMF de Salpeter, modelo SAGS. Se respeta el mismo código de colores que en el panel superior. Con líneas rojas continuas se delimita el intervalo de masas estelares definido en el criterio de M11. 

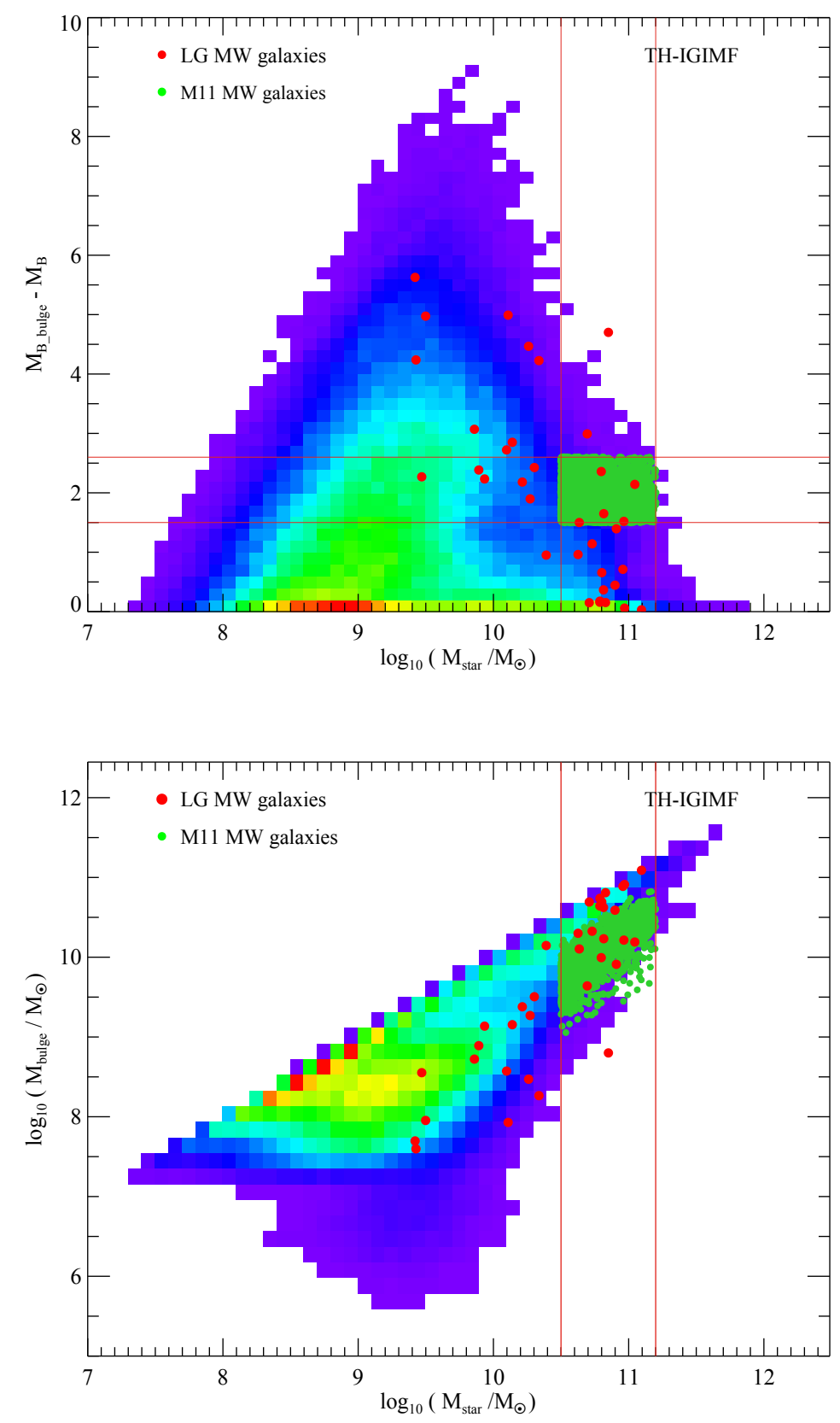

Figura 5.2: Panel superior: Similar al panel superior de la Fig. 5.1 pero para la muestra de galaxias del modelo SAGcon la TH-IGIMF, SAGTH5B2. Panel inferior: Similar al panel inferior de la Fig. 5.1 pero para la muestra de galaxias del modelo SAGTH5B2 con la TH-IGIMF. 
indicando que la masa estelar de estas galaxias se encuentra dominada por el bulbo. Las galaxias con masas menores al límite inferior según el criterio de M11 se corresponden con las galaxias tipo M31 de los pares seleccionados de acuerdo al criterio de G14, en acuerdo con las menores masas de halo de MO utilizado para su búsqueda. En el panel inferior de esta misma figura, se puede ver la masa de los bulbos de las galaxias de la simulación en función de su masa estelar. Las galaxias tipo VL están señaladas con el mismo código de colores que en el panel superior. Aquí se puede ver que los bulbos resultantes de seleccionar las galaxias tipo VL con el criterio de M11 tienen masas entre $5 \times 10^{8} \mathrm{M}_{\odot}$ y $5 \times 10^{9} \mathrm{M}_{\odot}$. Estos valores resultan más bajos en casi un orden de magnitud que las estimaciones observacionales de masas dinámicas del bulbo de la VL. En nuestro caso, suponemos que la masa dinámica es comparable a la masa estelar ya que la componente estelar domina la densidad total cerca del centro galáctico. Para comparar, consideramos los resultados obtenidos por Kent (1992) que indican que el bulbo de la VL tiene una masa dinámica de $10^{10} \mathrm{M}_{\odot}$, valor confirmado por el grupo de investigación de Manuela Zocalli utilizando datos del VVV (comunicación privada). Otros grupos encuentran masas dinámicas más altas, en un factor 2 (e.g. Sofue et al., $\left.2009, M_{\text {bulge-din }}=1,8 \times 10^{10} M_{\odot}\right)$. Por otro lado, las galaxias seleccionadas utilizando el criterio de pertenencia a sistemas análogos a grupos locales de G14 tienen bulbos en un rango muy amplio de masas. Un subconjunto de ellas tiene masas del orden de las estimaciones observacionales y 10 de ellas presentan bulbos con masas cercanas a la masa estelar total de la galaxia.

La Fig. 5.2 muestra los mismos resultados que en la Fig. 5.1 pero para la muestra de galaxias del modelo SAGTH5B2 que considera la TH-IGIMF. En este caso, como puede verse en el panel superior de la figura, hay una mayor cantidad de galaxias en sistemas análogos al LG y que además comparten el criterio de M11. A su vez, del análisis del gráfico en el panel inferior se desprende que las galaxias tipo VL seleccionadas a partir del criterio de M11 tienen bulbos con masas en el intervalo $5 \times 10^{9} \mathrm{M}_{\odot}$ y $5 \times 10^{10} \mathrm{M}_{\odot}$, consistentes en promedio con las estimaciones observacionales. Las galaxias tipo VL que cumplen el criterio de G14 nuevamente presentan bulbos en un intervalo amplio de masas. Sin embargo, un subgrupo de alrededor de 12 galaxias tiene masas muy similares a las estimaciones observadas. Las diferencias entre las masas estelares totales y de los bulbos de las galaxias tipo VL seleccionadas por distintos criterios, que se pueden apreciar en la Fig. 5.1, reflejan que la composición de la luz integrada del bulbo cuando se 
utiliza una IMF de Salpeter es diferente que cuando se utiliza la TH-IGIMF. El cociente de luminosidades (diferencia de magnitudes) exigido por el criterio de M11 selecciona galaxias con bulbos que no resultan tan masivos como la masa estimada del bulbo de la VL. En cambio, la composición estelar resultande de considerar una TH-IGIMF es diferente, con un mayor contenido de estrellas más masivas, lo que lleva a que los bulbos dentro del intervalo de magnitudes definido por M11 tengan una masa mayor y más acorde con la masa observada del bulbo.

\section{Poblaciones estelares en un modelo semianalítico}

Los modelos semianalíticos de formación de galaxias son muy versátiles para modificar implementaciones de fenómenos físicos y evaluar su impacto en las propiedades de las galaxias y sus componentes. Sin embargo, cuando se trata de la resolución de las componentes estelares, históricamente sólo se han considerado las componentes de las galaxias de manera monolítica. Se estudian las propiedades de los discos, de los bulbos y hasta halos estelares de las galaxias, pero siempre considerando el conjunto de estrellas que la componen y sus propiedades medias. En el presente trabajo, consideramos una manera novedosa de estudiar las poblaciones estelares (PEs) constitutivas del bulbo de galaxias tipo VL. La implementación que presentamos consiste en hacer el seguimiento de la masa y de las propiedades químicas de cada evento de formación estelar o acreción que ocurre y que produce una población estelar que pasa a formar parte del bulbo de las galaxias. Un evento de formación o acreción es singular en cuanto a las propiedades de sus estrellas, y acarrea la información de su lugar de origen.

Típicamente, cada galaxia del modelo con cociente entre la masa del bulbo y del disco (B/D) distinto de cero puede experimentar desde unos pocos a algunas decenas de eventos (brotes estelares o acreciones de estrellas) que contribuyen a la formación de su bulbo por todos los procesos posibles involucrados (ver Sección 5 del Capítulo 3). Una vez seleccionadas las galaxias tipo VL, apilamos la información de las propiedades de sus PEs, contenidas en arreglos de variables introducidas en el código, para obtener un mejor rango dinámico. Estas cantidades son pesadas estadísticamente considerando la masa de las PEs involucradas en cada evento. Diferenciamos las poblaciones según su origen para conocer con mayor detalle los procesos de formación del bulbo. De esta manera, podemos estudiar las propiedades de poblaciones acretadas por inestabilidad de 
disco (acretadas en ID), poblaciones formadas en brotes originados en inestabilidades de disco (brotes por ID), poblaciones acretadas en fusiones de galaxias (acretadas en fusiones) y poblaciones formadas en brotes disparados por fusiones de galaxias (brotes por fusiones). Los cuatro procesos mencionados son los únicos procesos modelados en SAG que dan lugar a la formación de los bulbos. Los dos primeros involcucran las estrellas y el gas de la propia galaxia que sufre la inestabilidad de disco, mientras que en los procesos de fusión también intervienen las estrellas y el gas de la galaxia satélite. Al considerar las PEs que pasan a formar parte del bulbo que son acretadas en fusiones o generadas en brotes por fusiones, no distinguimos entre fusiones mayores o menores, es decir, no tenemos en cuenta la relación entre las masas bariónicas de las galaxias involucradas, ni tampoco identificamos los casos de fusiones menores que dan lugar a brotes estelares, lo cual depende del contenido de gas frío de la galaxia central. En el caso de ocurrir una fusión mayor, cuando las masas estelares de la galaxia satélite acretada y la galaxia central son del mismo orden, el disco del remanente, que contiene el disco de la propia galaxia central, sufre un proceso de relajación dinámica y pasa a formar parte del esferoide formado en el evento. En estos casos, las PEs son computadas como originadas por acreción por fusión, aún cuando una fracción de las estrellas ya era parte de la galaxia.

\section{Resultados}

Una vez definidas las poblaciones estelares que forman los bulbos de galaxias tipo VL en el modelo SAG, comparamos resultados del modelo con datos observacionales para aportar información que ayude a la comprensión de la historia de formación del bulbo de nuestra galaxia y de cómo se relaciona ésto con las propiedades químicas del bulbo.

\subsection{Distribución de metalicidades de PEs del bulbo}

La distribución de metalicidades (DM) de las estrellas del bulbo es un observable fundamental para reproducir con el modelo de las PEs del bulbo de las galaxias implementado en SAG. Las DMs pueden ser obtenidas a partir de mediciones espectroscópicas de estrellas individuales del bulbo o, como detallaremos más adelante, a 
partir de mediciones fotométricas de las estrellas. El trabajo pionero en la determinación de abundancias de hierro en estrellas del bulbo fue realizado por Rich (1988). En los años posteriores fue posible obtener espectros de alta resolución (McWilliam \& Rich, 1994; Fulbright et al., 2006) de un reducido número de estrellas K gigantes, y ya en la siguiente década, con la construcción de instrumentos multi-objeto de alta resolución como FLAMES (Pasquini et al., 2003), ese número pudo ser extendido a $\sim 50$ (Zoccali et al., 2006). Paralelamente, se realizaron esfuerzos por obtener metalicidades de las estrellas M gigantes de menor temperatura. Rich \& Origlia (2005) reportan un análisis de abundancias de 14 estrellas de este tipo utilizando el instrumento NIRSPEC en el observatorio Keck y obtienen una metalicidad media similar a las obtenidas para las estrellas $\mathrm{K}$ gigantes del bulbo. El relevamiento VVV permitió tomar espectros de media resolución de 720 estrellas $\mathrm{K}$ gigantes en 4 campos a diferentes latitudes galácticas, $b=-12^{\circ}$, $b=-6^{\circ}$, la ventana de Baade y un campo que contiene al cúmulo globular NGC 6553 (Zoccali et al., 2008, Z08 de aquí en más). Más recientemente, con el relevamiento ARGOS (Freeman et al., 2013) se logró medir abundancias de alrededor de 14.000 estrellas gigantes pertenecientes al bulbo mediante el uso del espectrógrafo AAOmega en el telescopio Keck, aunque con una resolución más baja que con VVV $(R=11,000)$. Aunque la medición de estrellas gigantes en el rango del infrarrojo se presenta como la técnica más ampliamente utilizada, existen métodos para estudiar las propiedades de estrellas de menor masa en el bulbo. En una serie de trabajos, Bensby y colaboradores (e.g. Bensby et al., 2013) estudian las propiedades de 58 estrellas enanas y subgigantes del bulbo a partir de eventos de microlente gravitacional y obtienen distribuciones de metalicidad, entre otros observables. En esta sección, describimos las comparaciones realizadas entre las DMs de las PEs resultantes del modelo semianalítico SAG y dos distribuciones de metalicidades estimadas mediante espectroscopía y mediante un método fotométrico, y estudiamos el impacto del cambio de la IMF implementada en el modelo.

\section{Comparación con DMs espectroscópica}

Presentamos a continuación las comparaciones entre las DMs dadas por el modelo semianalítico SAG con observaciones espectroscópicas de Z08. Utilizamos los datos de los tres primeros campos de Z08 ya descriptos y dejamos de lado el campo de NGC 6553, ya que nos concentramos en las poblaciones estelares dispersas del bulbo. Esto 
reduce el número de estrellas con mediciones espectroscópicas a 507.

Los paneles superiores de la Fig. 5.3 muestran las DMs obtenida de las PEs apiladas de galaxias tipo VL del modelo SAGS, que considera una IMF de Salpeter, seleccionadas mediante el criterio de M11. En el panel superior izquierdo puede verse la DM total, sin discriminar por proceso de formación. La línea roja punteada representa el valor medio de la distribución del modelo, con un valor de $[\mathrm{Fe} / \mathrm{H}]=-1,2$, mientras que la línea punteada gris está ubicada en el valor medio de la distribución observada, con un valor de $[\mathrm{Fe} / \mathrm{H}]=-0,15$. La diferencia en los valores de cocientes de metalicidad es considerable. En el panel superior derecho se puede observar la DM desglosada en los diferentes mecanismos que dan lugar a las PEs del bulbo. Se observa que la mayor contribución a las PEs del bulbo corresponden a aquéllas acretadas en ID (histograma en línea azul), representando en promedio un $65,2 \%$ de la masa de los bulbos de las galaxias seleccionadas. Cabe destacar que las masas de los bulbos han sufrido efecto de reciclado de material estelar, mientras que ese efecto no es tenido en cuenta al diseñar los arreglos de variables con las PEs generadas por distintos procesos. El valor medio de la distribución de metalicidad de todas las PEs (línea punteada roja) no coincide con el máximo de la DM dada por PEs acretadas en ID sino que se encuentra corrido hacia valores levemente mayores de metalicidad debido a la contribución de las PEs generadas en brotes estelares tanto durante ID (histograma en línea celeste) como en fusiones (histograma en línea verde claro). Las PEs de estos procesos representan en promedio un $12,7 \%$ y un $13,7 \%$ de la masa total del bulbo de estas galaxias, respectivamente. Los mayores valores de metalicidad alcanzados por las PEs generadas en brotes estelares se debe a que el gas frío del disco que es transferido hacia el bulbo es consumido gradualmente (brotes estelares extendidos en el tiempo, ver Sección 9 del Capítulo 3), con lo cual puede ser contaminado por el material reciclado por estrellas formadas en sucesivos eventos de formación estelar. De este modo, se alcanzan metalicidades más altas que en PEs acretadas las cuales fueron originadas con anterioridad a partir de un gas menos enriquecido químicamente. En particular, las PEs generadas a partir de acreción en fusiones contienen la contribución de la masa estelar de galaxias satélites y su DM (histograma de línea verde oscuro) tiene el máximo ubicado hacia los valores de metalicidad más bajos presentes en las PEs $([\mathrm{Fe} / \mathrm{H}] \simeq-1,5)$ y representa una proporción marginal de la masa de las PEs en el bulbo, de 8,4\% del total.

Los paneles inferiores de la Fig. 5.3 muestran las DMs de las PEs de galaxias ob- 
tenidas del modelo SAGS que utiliza una IMF de Salpeter pero para galaxias tipo VL seleccionadas por el criterio de pertenencia a sistemas análogos al LG, según G14. Se puede observar que la metalicidad de las PEs de galaxias con similares condiciones de ambiente que las de una galaxia tipo VL, lo cual indicaría que están caracterizadas por un origen cosmológico en común con la VL, es mucho mayor que la obtenida para las PEs de galaxias seleccionadas con el criterio de M11. La metalicidad media de $[\mathrm{Fe} / \mathrm{H}]=0,057$ es incluso mayor a la observada en estrellas del bulbo por Z08.

Al comparar la importancia relativa de los distintos procesos en las DMs de galaxias seleccionadas por el criterio de G14 con aquéllas que satisfacen el critero de M11 (paneles inferior derecho y superior derecho, respectivamete), se observa un comportamiento similar en ambos casos, con un dominio de las PEs acretadas en ID, y una tendencia de las PEs generadas en brotes por fusión a alcanzar valores mayores de metalicidad. La diferencia más notoria se aprecia en la DM de PEs acretadas en fusiones que, en el caso de galaxias seleccionadas por el criterio de G14, presentan un máximo hacia valores más altos de metalicidad, opuesto a lo que sucede para galaxias seleccionadas por el criterio de M11. Teniendo en cuenta la contribución de la masa estelar de galaxias satélites a este grupo de PEs, este aspecto constituye una prueba más en favor a la distinta historia de formación y evolución que caracteriza a los dos grupos de galaxias.

La Fig. 5.4 muestra la DMs de las PEs en galaxias tipo VL del modelo SAGTH5B2 que considera la TH-IGIMF, seleccionadas mediante el criterio de M11 (paneles superiores) y el criterio de G14 (paneles inferiores). En el caso de galaxias tipo VL seleccionadas por el criterio de M11, la DM (histograma en línea roja) se encuentra en mejor acuerdo con la obtenida a partir de los datos espectroscópicos de Z08 que galaxias con una IMF de Salpeter, tal como puede apreciarse de comparar los paneles superiores izquierdos de las Fig. 5.4 y Fig. 5.3. La metalicidad media de esta distribución es $[\mathrm{Fe} / \mathrm{H}]=-0,49$ (línea punteada roja), mucho más cercana a la media de la distribución observada que en el modelo con una IMF de Salpeter $([\mathrm{Fe} / \mathrm{H}]=-0,14)$. En el panel superior derecho, se observan las contribuciones de cada proceso. La principal característica a notar es que, como en el caso de una IMF de Salpeter, existe una proporción mayoritaria de PEs provenientes del disco de la galaxia que han sido ya formadas y acretadas en ID (64,97\%), seguida de la contribución de estrellas generadas en brotes durante este mismo proceso $(23,19 \%)$. A diferencia de lo que ocurre para las galaxias tipo VL resultantes del modelo con IMF de Salpeter y seleccionadas con el 
criterio de M11, las distribuciones correspondientes a estos dos procesos comparten valores similares de metalicidades. Los valores más altos de metalicidad están dados por las PEs generadas durante brotes por fusión (histograma en línea verde claro, 10,56\%), que constituye una proporción aún menor de las PEs del bulbo.

Si bien la muestra de galaxias tipo VL seleccionadas por el criterio de M11 favorecen notoriamente la inclusión de una TH-IGIMF, en concordancia con los resultados descriptos en el Capítulo 4 al analizar la relación $[\alpha / \mathrm{Fe}]$-masa estelar de galaxias elípticas, la situación es claramente distinta al considerar el conjunto de galaxias seleccionadas por el criterio de G14. Los paneles inferiores de la Fig. 5.4 muestran las DM de las PEs de galaxias tipo VL obtenidas del modelo SAGTH5B2 que considera la TH-IGIMF pero seleccionadas según el criterio de G14; en el panel derecho, las PEs están diferenciadas por los procesos que les dan origen. En este caso se alcanza un mejor acuerdo con los datos observados de Z08, similar a lo que ocurre al considerar galaxias con IMF de Salpeter seleccionadas mediante el mismo criterio (ver paneles inferiores de la Fig. 5.3). Los resultados con selección G14 son más consistentes con las observaciones, pero cabe aclarar que con masas de bulbo con dispersión alta respecto a estimas para el bulbo de la VL. A primera vista, parecería que las historias de formación de las galaxias seleccionadas por el criterio de G14 son tales que se vuelven menos sensibles a la IMF adoptada. Sin embargo, al considerar los valores medios de las DMs, vemos que en el caso de un modelo con TH-IGIMF, el valor de $[\mathrm{Fe} / \mathrm{H}]=-0,09$ es más cercano al observado por Z08, favoreciendo también la inclusión de la TH-IGIMF.

La diferencia observada en las abundancias de hierro de las PEs en los modelos con diferente IMF se puede explicar por el mayor enriquecimiento químico experimentado por las galaxias en un modelo con TH-IGIMF, debido a las pendientes determinadas por la tasa de formación estelar de las mismas.

En el panel inferior de la Fig. 5.5 puede verse la tasa de formación estelar promedio de las galaxias tipo VL en función del tiempo en unidades de $\left[\mathrm{M}_{\odot} / \mathrm{yr}\right]$, y en la parte superior del gráfico se indica la pendiente $\alpha_{3}$ de la TH-IGIMF (Ver Sección. 8.2 del Capítulo 3). Cabe señalar que la pendiente $\alpha_{3}$ es válida para estrellas más masivas que $1 \mathrm{M}_{\odot}$, las cuales aportan la mayor parte de los metales al medio. Tanto las SNs Ia como las SNs II son más abundantes en una población estelar para pendientes más planas adoptada por las galaxias tipo VL seleccionadas por los criterios de M11 (línea verde oscuro sólida) y de grupos análogos al LG (línea roja punteada). La línea gris a rayas 

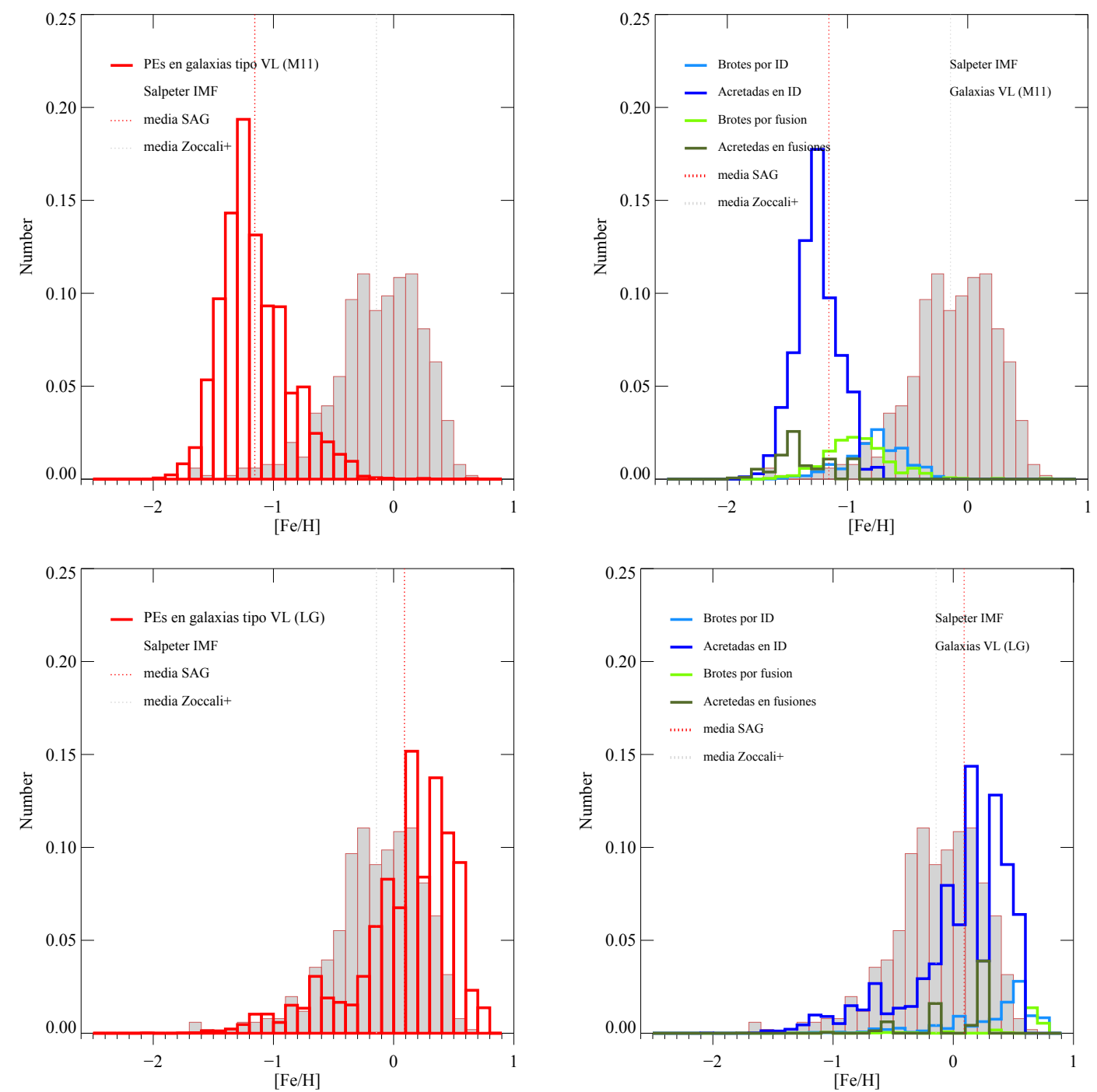

Figura 5.3: Panel superior izquierdo: Distribución de metalicidades de las PEs de galaxias tipo VL obtenidas del modelo SAGS que considera una IMF de Salpeter (histograma de líneas rojas); las galaxias tipo VL son seleccionadas de acuerdo al criterio de M11. Esta DM es comparada con la obtenida por Z08 a partir de datos espectroscópicos (histograma sombreado en gris). Las líneas roja y gris punteadas representan el valor medio de la metalicidad de las PEs del modelo y de la media de la distribución observada por Z08, respectivamente. Panel superior derecho: Similar al panel superior izquierdo pero con las DMs de las PEs del modelo diferenciadas por su origen según los colores indicados en las etiquetas. Paneles inferiores izquierdo y derecho: Similares a los paneles superiores izquierdo y derecho, respectivamente, pero para las galaxias seleccionadas con el criterio de de pertenencia a sistemas análogos a LG de G14 (ver Sección. 2). 

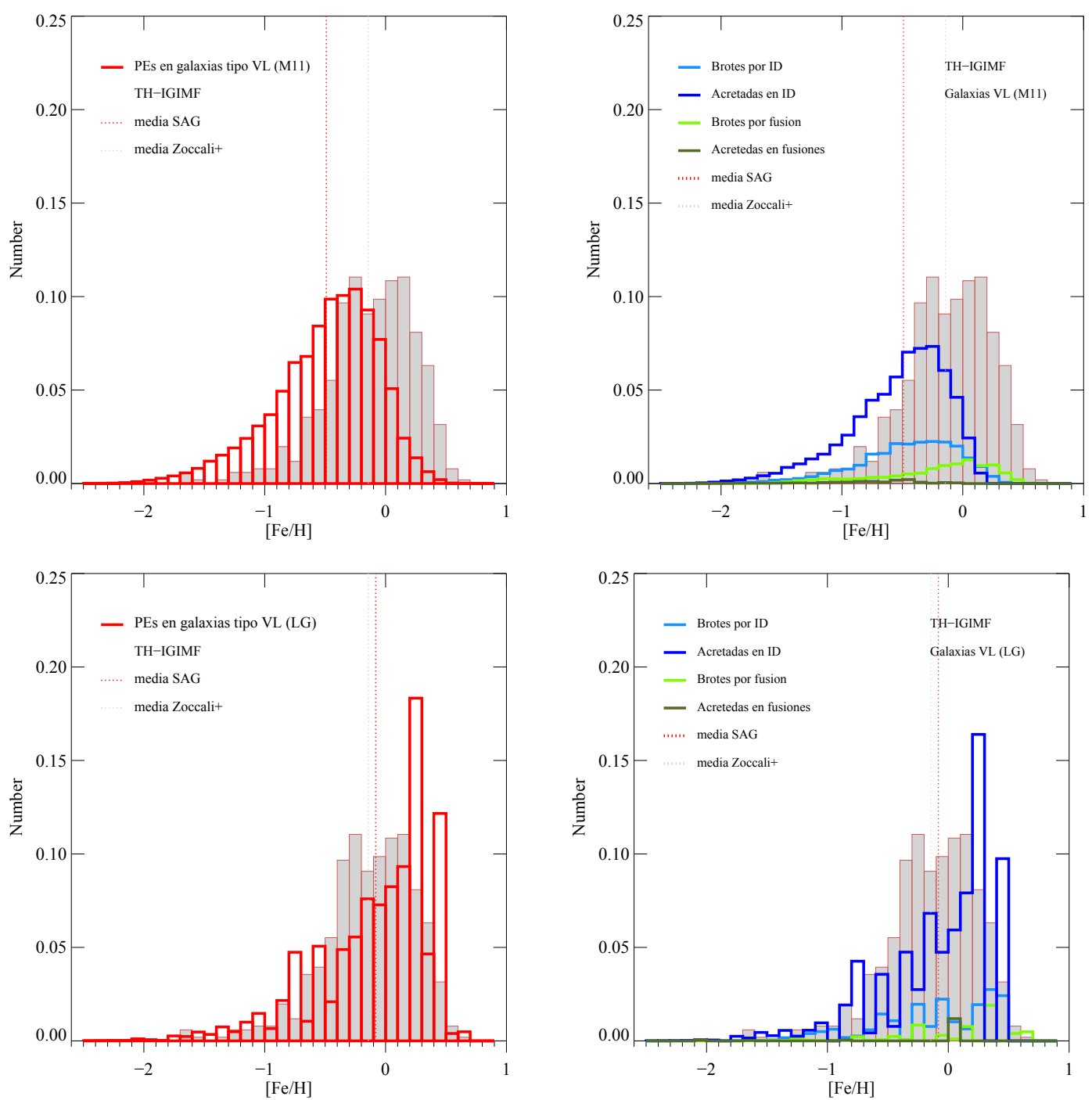

Figura 5.4: Panel superior izquierdo: Distribución de metalicidades de las PEs de galaxias tipo VL obtenidas del modelo SAGTH5B2 que considera la TH-IGIMF (histograma de líneas rojas); las galaxias tipo VL son seleccionadas de acuerdo al criterio de M11. Esta DM es comparada con la obtenida por Z08 a partir de datos espectroscópicos (histograma sombreado en gris). La líneas roja y gris punteadas representan el valor medio de la metalicidad de las PEs del modelo y la media de la distribución observada por Z08, respectivamente. Panel superior derecho: Similar al panel superior izquierdo pero con las DMs de las PEs del modelo diferenciadas por su origen según los colores indicados en las etiquetas. Paneles inferiores izquierdo y derecho: Similares a los paneles superiores izquierdo y derecho, respectivamente, pero para las galaxias seleccionadas con el criterio de de pertenencia a sistemas análogos a LG de G14 (ver Sección 2). 


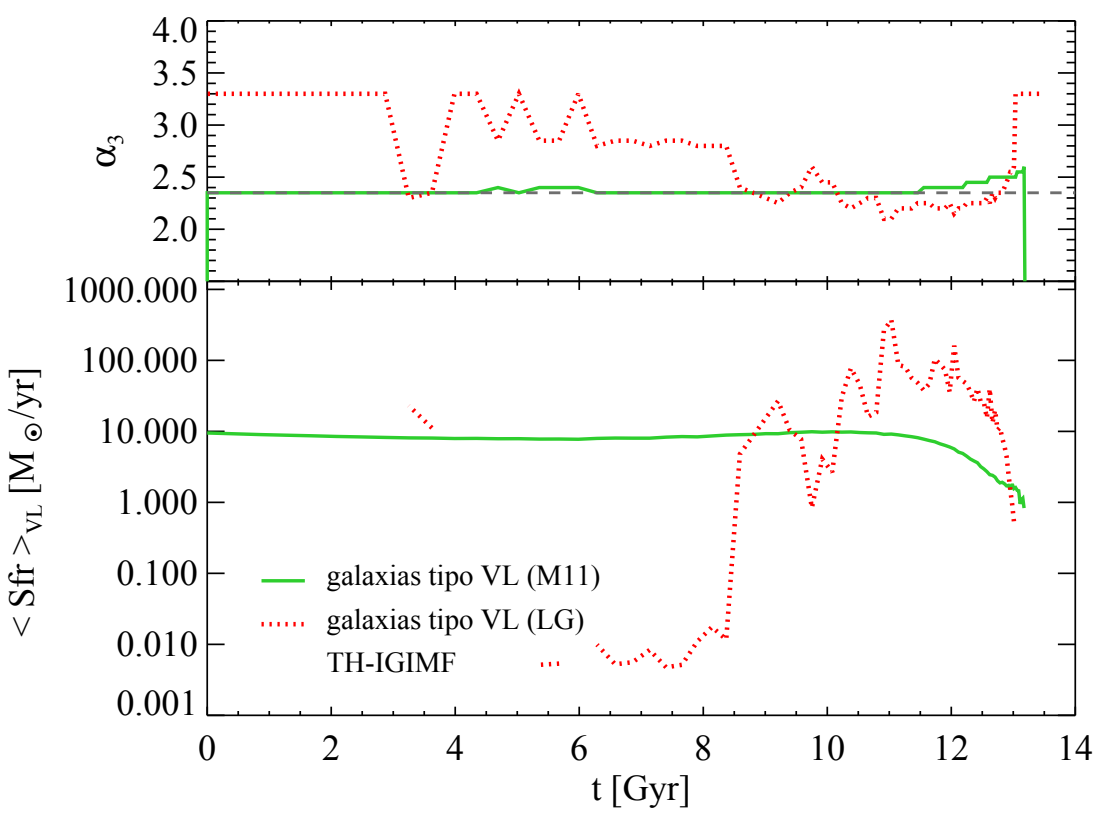

Figura 5.5: Panel superior: Pendiente $\alpha_{3}$ promedio de la TH-IGIMF adoptada por las galaxias tipo VL en el modelo SAGTH5B2 en función del lookback time. Se señala la pendiente de la IMF de Salpeter, con un valor de 2,35, con una línea gris. Las líneas roja punteada y sólida de color verde oscuro corresponden a galaxias tipo VL seleccionadas con el criterio de de pertenencia a grupos análogos al LG (G14) y con el criterio de M11, respectivamente. Panel inferior: Tasa de formación estelar promedio de las galaxias tipo VL seleccionadas con el criterios de M11 y de G14 (líneas verde oscuro y roja punteada, respectivamente) generadas por el modelo SAGTH5B2.

en el panel superior marca la pendiente de la IMF universal de Salpeter. Se puede ver que la pendiente adoptada por galaxias con TH-IGIMF y seleccionadas con el criterio de M11 tienen una pendiente $\alpha_{3}$ con un valor igual al valor de Salpeter durante la mayor parte del tiempo. Sin embargo, esto no indica que la distribución de masas inicial sea la misma que en el caso de Salpeter. La TH-IGIMF presenta un caída para estrellas de baja masa (ver Sección. 8.2 del Capítulo 3). La normalización de la TH-IGMF lleva a que exista un exceso de estrellas de mayor masa respecto a la IMF de Salpeter si $\alpha_{3}=2,35$, que producen una mayor eyección de metales al medio, generando un mayor grado de enriquecimiento químico de las estrellas y en particular, de hierro. En el caso de las galaxias tipo VL en grupos análogos al LG, puede observarse que en las épocas de mayor 
formación estelar la pendiente $\alpha_{3}$ es notoriamente más plana que la correspondiente a una IMF de Salpeter. Es interesante notar las diferentes historias de formación experimentadas por las galaxias tipo VL seleccionadas por los diferentes criterios. Por otro lado, las diferencias en las DM para los modelos con distinta IMF también se pueden atribuir a los diferentes valores de los parámetros libres obtenidos al calibrar los modelos con una IMF de Salpeter y con la TH-IGIMF. El valor de la eficiencia de feedback por $\mathrm{SN}$ en el bulbo es $\varepsilon_{\text {bulge }}=0,3163$ para el modelo con TH-IGIMF, un factor 4 veces mayor que el valor correspondiente al modelo con la IMF de Salpeter, caracterizado por $\varepsilon_{\text {bulge }}=0,0778$. Este hecho provoca que el gas frío en el bulbo disponible para la formación estelar en brotes sea eyectado y acretado con posterioridad, retrasando el proceso de formación estelar en el bulbo y alcanzando metalicidades cada vez mayores al ser contaminado por los sucesivos eventos de formación estelar. Esto se ve finalmente reflejado en las mayores metalicidades de las estrellas del bulbo, en mejor acuerdo con las abundancias observadas.

\section{Diagrama Color-Magnitud y DMs fotométrica}

Es un hecho establecido en la actualidad que existe un gradiente de metalicidades en el bulbo en dirección perpendicular al disco. Gonzalez et al. (2011, G11 de aquí en adelante) estudian la abundancia de elementos- $\alpha$ de la muestra de estrellas de Z08 descripta en la sección 4.1 y las tendencias que se evidencian con la latitud de los campos. Encuentran que las estrellas de mayor metalicidad y menor cociente $[\alpha / \mathrm{Fe}]$ se encuentran en el campo de latitud $b=-4^{\circ}$ y desaparecen para los campos de latitudes $b=-6^{\circ}$ y $b=-12^{\circ}$. En cambio, las estrellas de baja metalicidad están distribuidas uniformemente en los tres campos. Por otra parte, la densidad de estrellas es mucho más elevada a medida que nos acercamos al eje mayor del bulbo, o al disco de la galaxia. La muestra de estrellas con mediciones espectroscópicas, que tiene un número de estrellas similar en cada campo, presenta entonces un efecto de selección hacia estrellas de menor metalicidad, ya que no tiene en cuenta las densidades relativas de estrellas a diferentes latitudes. Donde se encuentra la mayor cantidad de estrellas en el bulbo, las estrellas son más metálicas. Si bien la DM espectroscópica obtenida por Z08 es precisa en cuanto a las mediciones, no representa de la mejor manera al bulbo como un todo. Teniendo en cuenta este defecto, decidimos realizar una comparación de las metalicidades del mo- 
delo con una muestra observada del bulbo más numerosa. Para realizarla, hicimos uso de la muestra fotométrica del VVV, que abarca alrededor de 320 grados cuadrados del bulbo de manera uniforme y contiene alrededor de $1,3 \times 10^{6}$ estrellas con mediciones de magnitudes $\mathrm{K}$ y J. El método para obtener metalicidades fotométricas consiste en realizar interpolaciones de los colores de las estrellas en la rama de las gigantes rojas entre líneas teóricas conocidas como "ridgelines", derivadas a partir de metalicidades precisas de estrellas de cúmulos globulares medidas con espectroscopía..

Mediante este método, desarrollado por Gonzalez et al. (2013, G13 de aquí en más), se obtuvieron las metalicidades para la muestra total de estrellas del VVV (Gonzalez et al., 2013). En el presente trabajo, se muestra por primera vez la DM de la muestra fotométrica total. La comparación con metalicidades del modelo no puede realizarse directamente con los datos obtenidos ya que, si bien el método se muestra efectivo para obtener metalicidades, los diagramas color-magnitud (DCM) dependen de otros parámetros de las estrellas, como la edad. Para realizar una correcta comparación, construimos DCMs sintéticos a partir de datos del modelo semianalítico SAG. Utilizamos el programa IAC-STAR (Aparicio \& Gallart, 2004), para el cual es necesario ingresar como datos de entrada la evolución de la metalicidad en el tiempo y la tasa de formación estelar. Calculamos estas cantidades para las PEs apiladas de galaxias tipo VL originadas en cada proceso. Seleccionamos las librerías de evolución estelar de TERAMO (Pietrinferni et al., 2004) y la librería de correcciones bolométricas de Girardi et al. (2002) como parámetros de entrada en IAC-STAR. El número total de estrellas que ingresamos para el cálculo fue de $10^{6}$. Usamos los valores por default en los parámetros de pérdida de masa en la rama de las gigantes rojas y en la rama asintótica. Para una mejor descripción del método de construcción de un DCM sintético, que excede el alcance de este trabajo, se dirige al lector al trabajo original de Aparicio \& Gallart (2004).

Es importante notar que, debido a las limitaciones de IAC-STAR, el diagrama colormagnitud sintético sólo puede ser generado para el caso de una IMF constante en el tiempo. No contamos con DCM para el modelo con una TH-IGIMF, sin embargo obtuvimos el DCM para el caso de IMF de Salpeter, con el objetivo de evaluar la capacidad del método fotométrico para obtener las metalicidades del modelo semianalítico en vez de considerar las provistas directamente por el código. Luego, aplicamos el mismo método que a las observaciones y recuperamos una distribución de metalicidades para las PEs de las galaxias tipo VL del modelo. En la Fig. 5.6 se muestra, como ejemplo, el 
DCM obtenido para las PEs de galaxias tipo VL seleccionadas por el criterio de M11. obtenidas del modelo SAGS caracterizado por una IMF de Salpeter. Se distinguen con distintos colores los CMDs correspondientes a PEs generadas por cada uno de los cuatro procesos de formación de bulbo considerados en SAG.

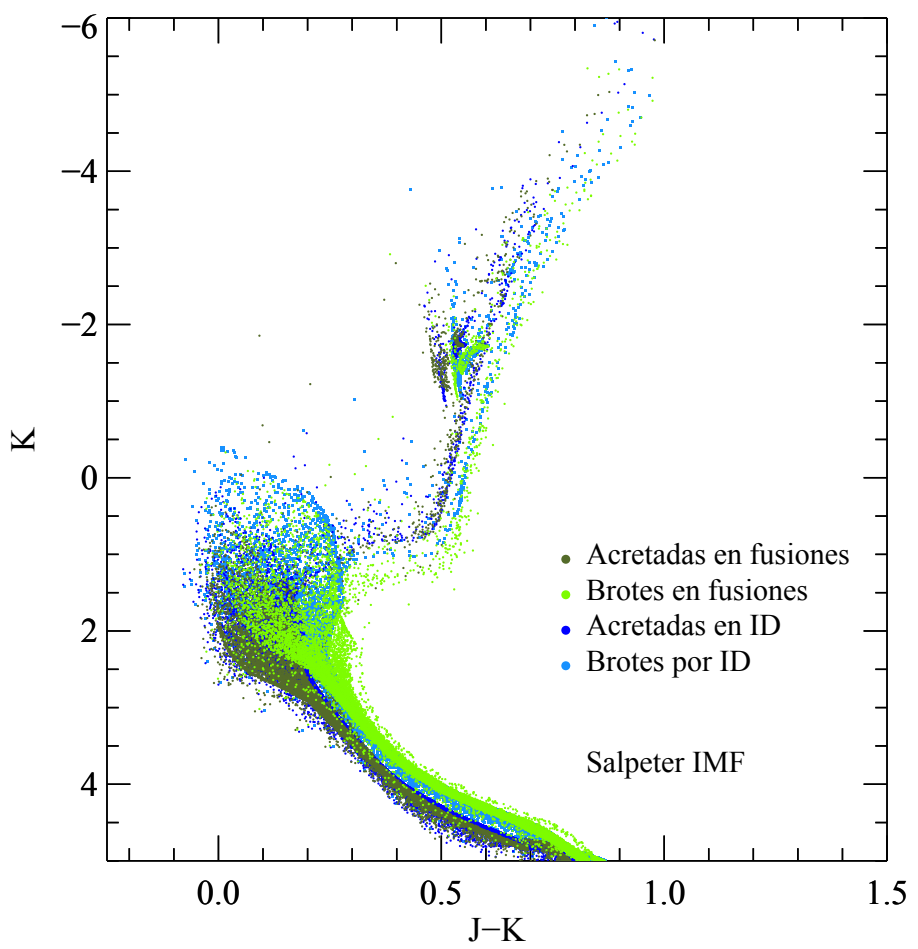

Figura 5.6: Diagrama color-magnitud para galaxias tipo VL obtenidas a partir del modelo SAGS que considera la IMF de Salpeter seleccionadas según el criterio de M11. Este DCM sintético es generado a partir de datos de evolución de la tasa de formación estelar y de acreción, y de la evolución de la metalicidad de los distintos procesos de formación de bulbo en SAG.

En la Fig. 5.7 mostramos, con un histograma sombreado gris, la distribución de metalicidades fotométricas cortesía de Oscar Gonzalez, coautor del trabajo en preparación cuyo contenido refleja los resultados de este capítulo de la tesis. La distribución fue estimada con datos fotométricos de más de un millón de estrellas del bulbo obtenidas mediante el survey de galaxias VVV. Como era de esperarse por los argumentos esgrimidos al comienzo de la sección, al considerar un gran número de estrellas en el extenso campo de VVV encontramos que la DM está más corrida hacia estrellas con mayor me- 

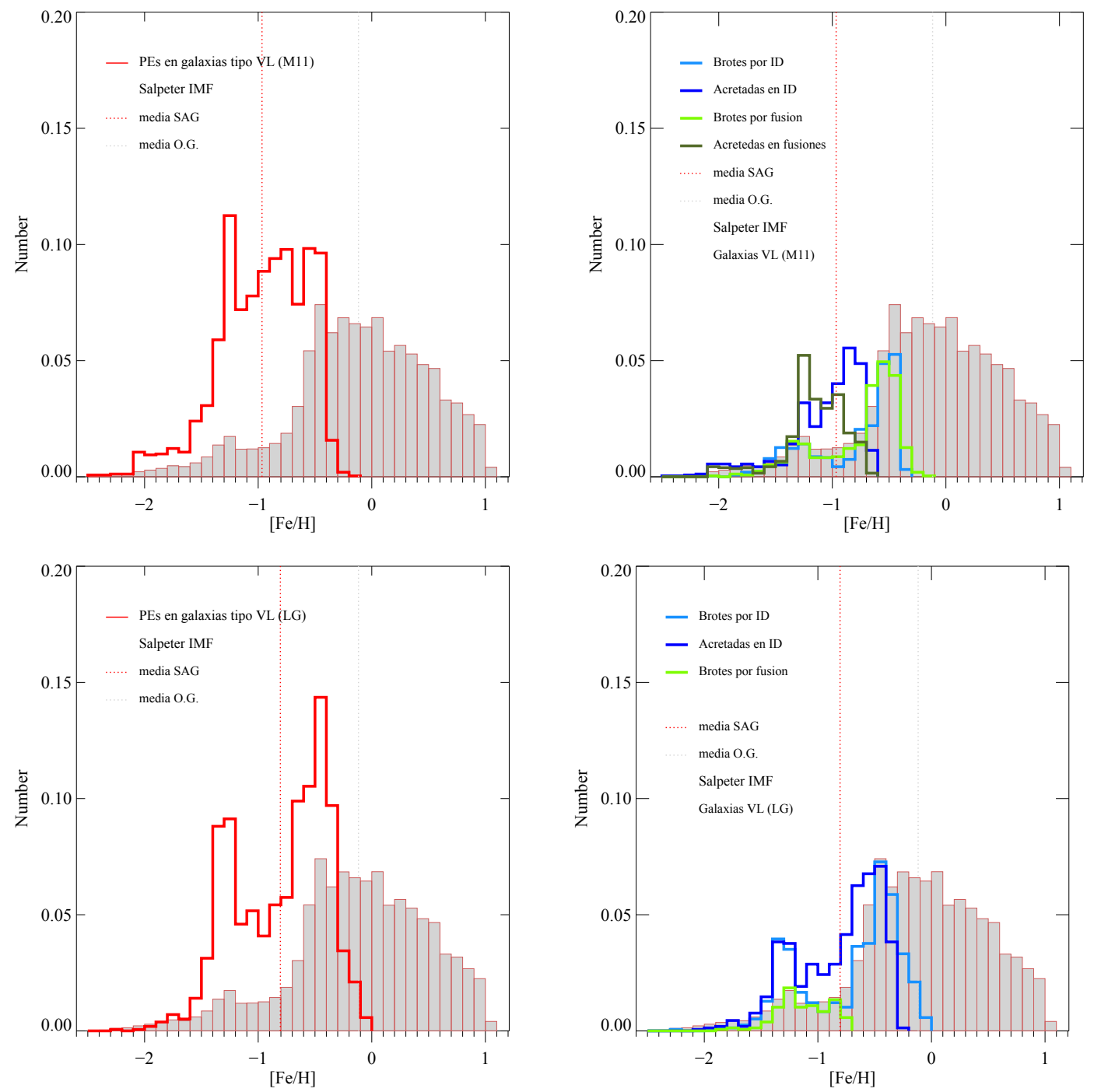

Figura 5.7: Panel superior izquierdo: Distribución de metalicidades de las poblaciones estelares de galaxias tipo VL seleccionadas con el criterio de M11 generadas a partir del modelo SAGS que considera una IMF de Salpeter (histograma en línea roja). La DM es obtenida a partir de la aplicación del método de G13 y es comparada con los resultados observacionales fotométricos procesados por Oscar González (histograma sombreado gris; comunicación privada). Panel superior derecho: Distribución de metalicidedes de PEs con distinto origen obtenidas del modelo SAGS (histogramas de diferentes colores) a partir de la aplicación del método de G13, comparadas con la DM obtenida a partir de los datos fotométricos de O. González (histograma sombreado gris). Panel inferior izquierdo: Similar al panel superior izquierdo, pero para las galaxias tipo VL del modelo SAGS seleccionadas con el criterio de pertenencia a grupos análogos al LG. Panel inferior derecho: Similar al panel superior derecho, pero para galaxias tipo VL del modelo SAGS seleccionadas con el criterio de pertenenecia grupos análogos al LG. No incluimos las PEs acretadas en fusiones en el análisis por contribuir con una proporción despreciable al total de las PEs. 
talicidad que la DM espectroscópica (ver Fig. 5.3). En el panel superior izquierdo mostramos la distribución de metalicidad total, es decir, de todas las PEs de los bulbos, de galaxias tipo VL generadas por el modelo SAGS caracterizado por una IMF de Salpeter y seleccionadas por el criterio de M11. En el panel superior derecho identificamos con el mismo código de colores que en la Fig. 5.3 las metalicidades fotométricas de las PEs originadas mediante los cuatro procesos de formación del bulbo considerados en el modelo semianalítico. Las líneas punteadas rojas y grises identifican los valores medios de las DMs modeladas $([\mathrm{Fe} / \mathrm{H}]=-0,97)$ y observadas $([\mathrm{Fe} / \mathrm{H}]=-0,18)$, respectivamente. Si bien la DM fotométrica se extiende hacia valores más altos de metalicidad, el valor medio de la distribución es similar al correspondiente a la DM espectroscópica. Por otro lado, la DM modelada obtenida a partir del método de metalicidades fotométricas de G13 tiene una media con un valor de metalicidad mayor que aquél correspondiente a la media de la DM dada directamente por el modelo semianalítico, siendo menos concentrada alrededor de este valor, tal como puede apreciarse al comparar los paneles superiores de las Figs. 5.7 y 5.3. Esta diferencia entre las DM modeladas puede deberse a las suposiciones inherentes al método de G13, tales como las librerías de evolución estelar y de correcciones bolométricas, y tasas de pérdidas de masa de las estrellas. Sin embargo, estas diferencias no cambian las conclusiones generales ya obtenidas. La aplicación del método fotométrico evidencia un desfasaje en valores de metalicidad entre las DM modeladas y observadas cuando se consideran galaxias obtenidas a partir de un modelo con IMF de Salpeter y seleccionadas con el criterio de M11. En el panel superior derecho se pueden observar las contribuciones de las estrellas generadas en los distintos procesos de formación del bulbo. El método descripto para obtener metalicidades fotométricas del modelo no puede reproducir las contribuciones relativas reales, ya que la distribución de metalidades de cada proceso es normalizada por separado al construir el CMD sintético.

En la Fig. 5.7 también se muestran las distribuciones para el total de las PEs de galaxias VL seleccionadas con el criterio de G14 y dividida por los diferentes procesos (paneles inferior izquierdo e inferior derecho, respectivamente). No se incluyen las PEs originadas en acreciones por fusiones, ya que la masa relativa es muy pequeña. Se observa en el panel inferior izquierdo que la metalicidad media de las estrellas del CMD sintético generado con galaxias tipo VL seleccionadas por el criterio de pertenencia a sistemas análogos al $\mathrm{LG}([\mathrm{Fe} / \mathrm{H}]=-0,8)$, es mayor que la metalicidad media 
de las estrellas del CMD sintético generado con datos del modelo SAGS con galaxias VL seleccionadas utilizando el criterio de M11, como se puede ver comparando con el panel superior izquierdo. Sin embargo, al comparar con el panel inferior izquierdo de la Fig. 5.3, se aprecia que el valor medio de las metalicidades obtenidas con el método fotométrico a partir de datos del modelo es más bajo que aquél obtenido directamente de las metalicidades del modelo. Además, no se logra recuperar la media de la metalicidad observada derivada de los datos fotométricos, como ocurre para este mismo grupo de galaxias cuando se comparan las metalicidades espectroscópicas y las provistas directamente por el modelo (paneles inferiores de la Fig. 5.3). A pesar de esta discrepancia, es interesante notar que, si bien las metalicidades obtenidas por el método fotométrico de G13 no alcanzan los valores de metalicidad más altos observados, la DM presenta una estrucutura bimodal donde los valores de los máximos coinciden con las metalicidades correspondientes a la DM observada.

El método fotométrico presentado en G13 fue probado mediante comparaciones estadísticas sobre un mismo conjunto de estrellas dando como resultado que podía considerarse equivalente al método espectroscópico (Gonzalez et al., 2013). Los detalles del modelado del IAC-STAR escapan al desarrollo de esta tesis, pudiéndose hallar en ellos la razón de la discrepancia entre ambos métodos para el conjunto de galaxias seleccionadas con el critero de G14. Una posible interpretación es que las edades de las poblaciones acretadas por inestabilidades de disco estén subestimadas y produzcan una mala determinación de la metalicidad fotométrica. Se cuenta con cotas inferiores de las mismas, porque la edad de estas PEs se calcula a partir del momento de la acreción de estrellas, cuando es posible guardar sus propiedades, y no del momento en que las mismas se forman. Las estrellas generadas podrían ocupar lugares incorrectos en los DCM y conducir a determinaciones de metalicidad más bajas. Este bias en las edades podría estar presente también en las galaxias tipo VL seleccionadas con el criterio de M11, sin embargo, las mismas no muestran una diferencia tan notoria entre el valor medio de la metalicidad dada directamente por el modelo y la derivada por el método fotométrico de G13. Si aceptamos que las metalicidades derivadas del método fotométrico de G13 son correctas, la inconsistencia entre los resultados encontrados para un modelo con IMF de Salpeter podría proceder de las diferencias evolutivas de las propias galaxias tipo VL pertenecientes a dos conjuntos distintos según el criterio de selección adoptado, tal como puede apreciarse de la evolución de sus tasas de formación estelar mostrada en la 
Fig. 5.5. Estas diferencias en la evolución de sus propiedades son evidentes al considerar las metalicidades provistas directamente por el modelo semianalítico, pero se diluyen al pasar por el "filtro" del cálculo de las metalicidades por el método fotométrico. Estos resultados ponen en tela de juicio la interpretación de resultados observacionales, ya que de una muestra de galaxias con propiedades químicas claramente distintas pueden obtenerse DMs similares al derivar las mismas mediante el método fotométrico que considera como datos de entrada otras propiedades de las galaxias, junto con una serie de suposiciones adicionales inherentes al modelo.

\subsection{Abundancias de elementos- $\alpha$ en las PEs del bulbo}

Las abundancias de elementos $\alpha$ de las estrellas pertenecientes al bulbo de nuestra galaxia pueden resultar una fuente de información valiosa a la hora de estudiar la historia de formación de esta componente. Como se desarrolló en el Capítulo 4, los cocientes de abundancias de estos elementos respecto al hierro pueden utilizarse como indicadores de escalas de tiempo de formación de un sistema estelar si suponemos una IMF universal, o bien pueden interpretarse como resultado de la ocurrencia de una IMF con diferentes pendientes durante la formación estelar del mismo. Distintos trabajos observacionales destinados a las abundancias de estrellas del bulbo se dedican en parte al estudio de las abundancias de elementos $\alpha$. McWilliam \& Rich (1994) encuentran, en su muestra de gigantes $\mathrm{K}$ del bulbo, sobreabundancias de elementos $\alpha$ respecto a las solares para el $\mathrm{Mg}$ y el Ti para todo el intervalo medido de $[\mathrm{Fe} / \mathrm{H}]$ mientras que el $\mathrm{Ca}$ y el Si muestran abundancias solares para cocientes $[\mathrm{Fe} / \mathrm{H}]>-0,2$, de manera similar a las estrellas del disco. Ellos especulan que el exceso de elementos $\alpha$ puede reflejar un proceso de enriquecimiento común de las galaxias elípticas con el bulbo. Zoccali et al. (2006) miden las abundancias de oxígeno de 50 estrellas $\mathrm{K}$ gigantes del bulbo y encuentran que las estrellas tienen cocientes $[\mathrm{O} / \mathrm{Fe}]$ suprasolares y más altos que aquéllos de las estrellas del disco grueso, que a su vez están más enriquecidas en elementos $\alpha$ que las estrellas del disco fino (Bensby et al., 2004). Ellos también consideran que la evidencia encontrada le da apoyo a la visión del bulbo como un esferoide viejo de formación rápida, similar a como se suele considerar a las galaxias elípticas y asimilable a la condición de bulbo "clásico". G11 estudian los cocientes de abundancias de $[\alpha / \mathrm{Fe}]$ de 474 estrellas de la rama de las gigantes en el bulbo de la VL y 176 más pertenecientes 
a NGC 6553 utilizando el instrumento FLAMES-GIRAFFE en el VLT. La muestra es aquélla analizada en Z08. A la vez, reanalizaron la muestra del disco grueso y fino de Alves-Brito et al. (2010) y obtuvieron abundancias para estas componentes también, de manera homogénea, para investigar el origen de las diferentes componentes. Encuentran que las estrellas de alta metalicidad tienen bajos cocientes de $[\alpha / \mathrm{Fe}]$ y se encuentran exclusivamente a valores bajos de latitud $\left(b=-4^{\circ}\right)$, mientras que las estrellas de baja metalicidad están distribuidas de manera homogénea en el bulbo y tienen propiedades idénticas a las estrellas del disco grueso. Concluyen que la población de estrellas pobres en metales tienen cocientes de abundancias $[\alpha / \mathrm{Fe}]$ muy suprasolares y mayores a los del disco fino, y que tendrían origen en una formación rápida consistente con un bulbo clásico. Consideran además que la existencia de un gradiente de metalicidades observado en los campos a diferentes latitudes del bulbo apoya esta interpretación. Luego, afirman que la presencia de estrellas muy metálicas a valores de latitud bajos y su desaparición para otras latitudes más alejadas del eje mayor del bulbo, como también su bajo enriquecimiento en elementos $\alpha$, indican que el origen de esta población es consistente con la formación de un pseudo-bulbo por evolución secular. G11 también buscan un gradiente en las abundancias de elementos $\alpha$ y curiosamente no encuentran ninguno, como cabría esperar si existieran dos poblaciones netamente diferenciadas.

El cálculo de las abundancias de elementos $\alpha$ en el modelo se realizó como se detalla en la Sección 2 del Capítulo 3, pero en este capítulo sólo consideramos en la suma de la ec. 4.1 a las abundancias de los elementos $\mathrm{Mg}, \mathrm{Ca}$, Si. La comparación de los resultados del modelo se realiza con los datos de G11, que sólo miden las abundancias de estos tres elementos y el Ti. No consideramos este último elemento debido a la falta de yields de Ti en nuestro modelo.

En la Fig. 5.8, mostramos el cociente de abundancias $[\alpha / \mathrm{Fe}]$ en función del cociente $[\mathrm{Fe} / \mathrm{H}]$ de las PEs del modelo con distintas IMFs. En la figura superior mostramos las abundancias de las PEs en el modelo SAGS, caracterizada por una IMF de Salpeter. En círculos rojos están representados las abundancias estimadas por G11 de la muestra de estrellas del bulbo de Z08. Los diferentes colores en cada uno de los paneles de la figura (verde oscuro, verde claro, azul y celeste) indican los distintos orígenes de las PEs del modelo, cuyas galaxias fueron seleccionadas con el criterio de M11; las mismas son comparadas con las PEs de galaxias tipo VL seleccionadas de acuerdo al criterio que considera la pertenencia a grupos análogos al LG, las cuales se representan en gris. Con- 

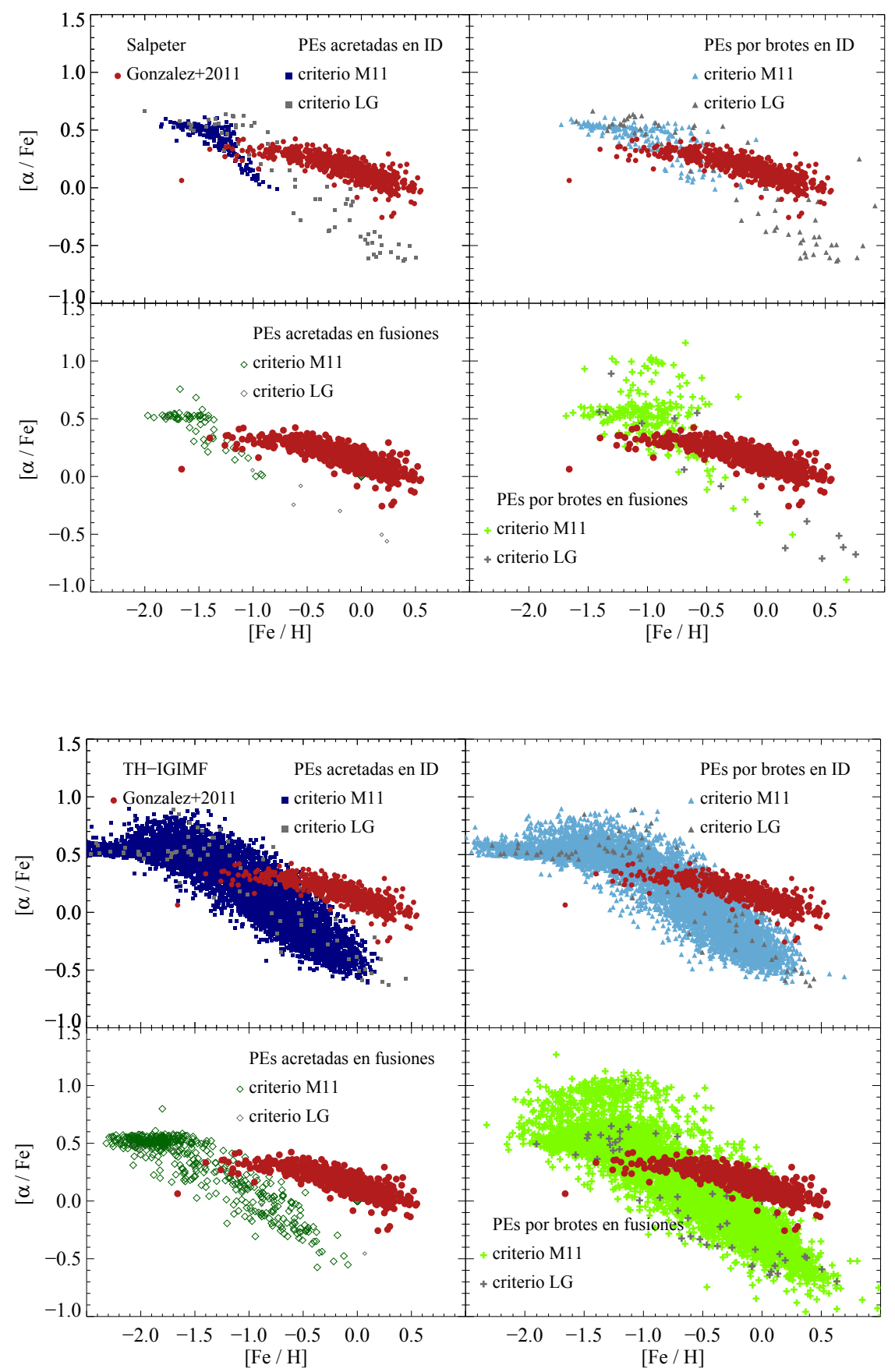

Figura 5.8: Figura superior: Abundancias de elementos $\alpha$ respecto a las abundancias de hierro en función de las metalicidades $[\mathrm{Fe} / \mathrm{H}]$ para $\mathrm{PEs}$ del modelo utilizando una IMF de Salpeter, SAGS. En rojo se muestran las abundancias de estrellas medidas por Gonzalez et al. (2011) en la muestra de estrellas de Z08 utilizada. Los diferentes paneles muestran las PEs con distinto origen, destacando con diferentes colores (verde oscuro, verde claro, azul y celeste) las galaxias tipo VL seleccionadas con el criterio de M11, y en gris aquéllas seleccionadas por el criterio de pertenenecia a sistemas análogos de grupos locales de G14 (LG). Figura inferior: Igual a la figura superior, pero para las PEs de galaxias tipo VL del modelo SAGTH5B2 que considera una TH-IGIMF. 
siderando las PEs de las galaxias tipo VL seleccionadas según el criterio de M11, puede verse que el desfasaje de las secuencias de abundancias modelada y observada evidencia la falta de hierro en las PEs, que ya se había notado en las DMs mostradas en la Sección 4.1. Sin embargo, puede observarse un comportamiento similar de las abundancias de PEs en el modelo con respecto a las abundancias observadas, en el sentido de que existe una secuencia plana de enriquecimiento de elementos $\alpha$ para PEs (estrellas) de menor metalicidad que luego decrece a medida que las PEs son más metálicas. Usualmente, este tipo de diagramas se interpreta como una secuencia evolutiva de las estrellas. Las estrellas de menor metalicidad son las primeras en formarse, y se enriquecen de los elementos $\alpha$ que las estrellas masivas eyectan al medio interestelar al terminar su vida, en escalas de tiempo cortas. A medida que pasa el tiempo, el gas se vuelve cada vez más químicamente enriquecido, no sólo por elementos $\alpha$ sino también por Fe y elementos del pico de hierro, dado que comienzan a explotar las SNs Ia, de mayor tiempo de vida $\mathrm{y}$ en cuyos yields predominan estos últimos elementos; las nuevas generaciones de estrellas van acumulando así una mayor cantidad de hierro. Si bien el comportamiento es similar, con un período de enriquecimiento de elementos $\alpha$, la pendiente mostrada por las PEs del modelo resulta más empinada que lo indicado por las observaciones para cualesquiera de los procesos de formación de bulbo considerados, como se puede apreciar de los distintos paneles de la figura. Esto puede indicar que: o bien, la formación estelar en el bulbo de las galaxias tipo VL del modelo en cada evento de formación es más prolongada que lo que fue la formación del bulbo de la VL, dando tiempo a que un número mayor de SNs Ia exploten, o los tiempos de retardo en la explosión (delay times) de las SNs Ia que resultan de la simulación son muy cortos y la rapidez con que las estrellas se enriquecen de hierro está sobrestimada. Ahora bien, esta última posibilidad va en contra de lo sugerido tanto por la falta general de hierro en las subpoblaciones de galaxias tipo VL seleccionadas por el criterio de M11 (para ambas suposiciones de IMF, IMF de Salpeter o TH-IGIMF), como por la falta de hierro en galaxias elípticas, que se evidencia en valores más altos de lo observado de los cocientes $[\alpha / \mathrm{Fe}]$ analizados en el Capítulo 4. En ese capítulo, se estudiaron las escalas de tiempo de formación de las galaxias elípticas y se llegó a la conclusión de que las escalas de tiempo de formación de estas galaxias es mayor que la esperada para este tipo de galaxias, y su patrón de enriquecimiento de elementos $\alpha$ no se puede explicar por la celeridad en su formación, y sí podía reproducirse gracias a la implementación de la TH-IGIMF. Dado que, por 
definición en el modelo, los bulbos de las galaxias comparten los mecanismos de formación con las galaxias elípticas, una formación estelar más lenta para los bulbos sería esperable, dados los resultados encontrados para galaxias elípticas. Por otro lado, la implementación de diferentes delay times de SNs Ia es un paso obligado en el modelado químico que, sin embargo, está fuera del alcance de esta investigación y será incluido en trabajos posteriores.

Enfocándonos en el comportamiento de la relación $[\alpha / \mathrm{Fe}]$ vs $[\mathrm{Fe} / \mathrm{H}]$ de las PEs de las galaxias tipo VL seleccionadas por distintos criterios, en general, se observa que las PEs de las galaxias tipo VL en grupos análogos al LG (símbolos grises) están ubicadas en un intervalo mayor de $[\mathrm{Fe} / \mathrm{H}]$ que aquéllas seleccionadas con el criterio M11 (símbolos de colores), y no presentan el desfasaje en hierro con respecto a las observaciones, como ya podía verse en las DMs de la Sección 4.1. Sin embargo, también muestran una mayor pendiente de decrecimiento de $[\alpha / \mathrm{Fe}]$ con $[\mathrm{Fe} / \mathrm{H}]$ que las correspondientes a las observaciones, indicando que esta característica no depende del ambiente en el cual se encuentran las galaxias, como sí lo hace la metalicidad total de las PEs.

El gráfico inferior de la Fig. 5.8 es similar al superior, pero para las galaxias del modelo SAGTH5B2. La primer característica a notar es la mayor cantidad de PEs producidas en galaxias tipo VL para el modelo con una TH-IGIMF, seleccionadas tanto por el criterio de M11 (símbolos en colores) como por el de G14 (símbolos grises). Al analizar previamente la Fig. 5.2, podía observarse que la masa estelar de los bulbos en este modelo es mayor que cuando se utiliza una IMF de Salpeter (Fig. 5.1) y, por lo tanto, están formados por una mayor cantidad de PEs. Asimismo, la cantidad de galaxias tipo VL es mayor para el modelo con una TH-IGIMF que para el modelo con Salpeter. El segundo aspecto que caracteriza la relación modelada es que las metalicidad de las PEs de galaxias tipo VL seleccionadas según el criterio de M11 alcanzan valores más altos superponiéndose con las correspondientes a PEs de galaxias tipo VL seleccionadas por el criterio G14, tal como se aprecia de la DMs mostradas en la Fig. 5.4. Es importante notar que la pendiente de la relación $[\alpha / \mathrm{Fe}]$ vs. $[\mathrm{Fe} / \mathrm{H}]$ no depende de la IMF adoptada ni del criterio de selección usado para identificar galaxias tipo VL.

Volviendo al tema de la diferencia entre la masa de los bulbos obtenidas a partir de distintas IMFs, notamos que la misma radica en la historia de enriquecimiento químico de las galaxias cuando se utilizan las diferentes IMFs. Como ya fue mencionado anteriormente, las pendientes planas de la TH-IGIMF en las épocas de mayor formación 
estelar producen un mayor enriquecimiento en metales, que a su vez logra aumentar la formación estelar, ya que la tasa de enfriamiento del gas depende de la metalicidad del mismo. La otra razón, ya mencionada, es la existencia de una mayor cantidad de retroalimentación por SNs. La cantidad mayor de estrellas de gran masa presentes en el bulbo del modelo con TH-IGIMF, junto al valor más alto de eficiencia obtenido en la calibración de este modelo produce un efecto mucho más fuerte de retroalimentación, prolongando la formación estelar en el tiempo. Estos efectos se hacen gráficos en la siguiente sección, en la que estudiamos las historias de formación estelar y de acreción de los bulbos de las galaxias tipo VL, junto a la evolución de su metalicidad.

\subsection{Historias de formación y evolución de la metalicidad}

La capacidad de conocer las propiedades de las PEs en un modelo semianalítico de formación de galaxias permite estudiar la historia de formación del bulbo y la evolución en su metalicidad, ya que la información es guardada a cada paso de la simulación, cuando ocurre un evento de formación estelar o de acreción. Conocer esta información, junto con las propiedades de las subpoblaciones en el presente, permite validar las hipótesis sobre la formación del bulbo.

El panel superior izquierdo de la Fig. 5.9 muestra la tasa de formación estelar para los casos de PEs originadas en brotes, ya sea por inestabilidad de disco (azul claro) o por fusiones (verde claro), en función del lookback time, para galaxias tipo VL generadas a partir del modelo con la IMF de Salpeter, SAGS, y seleccionadas de acuerdo al criterio de M11. A su vez, está indicada la tasa de acreción de masa de PEs hacia el bulbo por inestabilidad de disco (azul oscuro) y fusiones (verde oscuro). En el caso de las galaxias acretadas, no se cuenta con la información detallada de las galaxias de las cuales provienen que permita conocer la tasa de formación estelar de la galaxia en la cual se formaron, sin embargo podemos conocer su tasa de acreción. En el caso de las inestabilidades de disco, podemos conocer el instante en que las estrellas pasaron a ser parte del bulbo, pero no cuándo se formaron, con lo cual sólo tenemos una cota inferior de su edad.

Se observa claramente en el gráfico el predominio de PEs provenientes del disco de la misma galaxia por inestabilidades gravitacionales. Vale la pena notar el intervalo de tiempo corto en el cual se forman las poblaciones estelares en este caso y la edad 

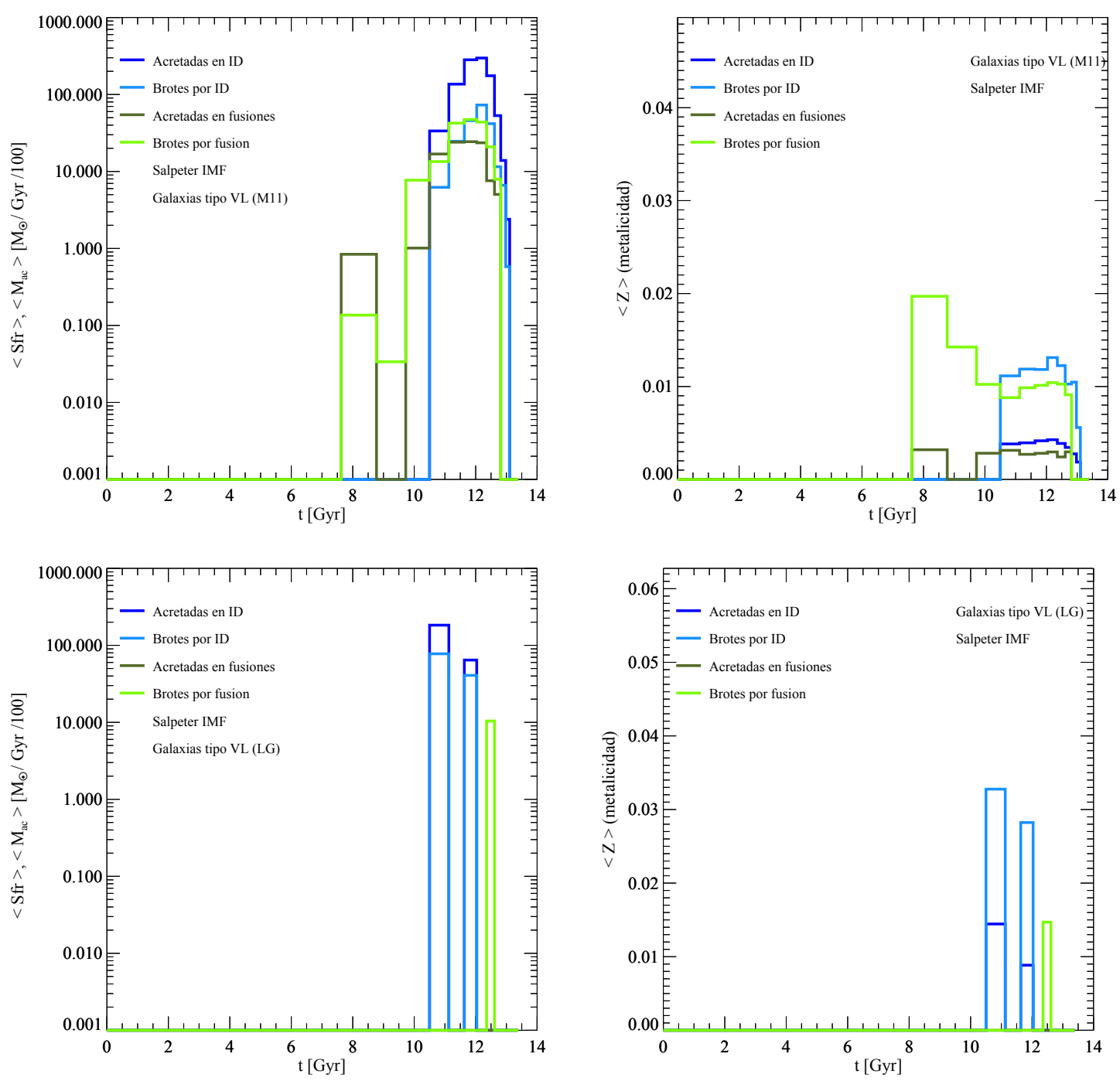

Figura 5.9: Panel superior izquierdo: Tasa de formación estelar promediada cada 5 instantes de salida de la simulación (snapshots) en brotes por fusiones e inestabilidades de disco, y cantidad de masa acretada promediada cada 5 snapshots de estrellas acretadas en fusiones e inestabilidades de disco. Todas estas cantidades son graficadas en función del lookback time. El código de colores está indicado en las etiquetas. Los resultados son para el modelo con una IMF de Salpeter, SAGS, y el criterio de selección de galaxias tipo VL de M11. Panel superior derecho: Metalicidad de las poblaciones estelares formadas, promediada cada 5 snapshots en función del lookback time. Los resultados son para galaxias tipo VL generadas por el modelo SAGS y seleccionadas según el criterio de M11. Paneles inferiores izquierdo y derecho: Similares a los paneles superiores izquierdo y derecho, respectivamente, pero para las galaxias tipo VL seleccionadas según el criterio de G14. 

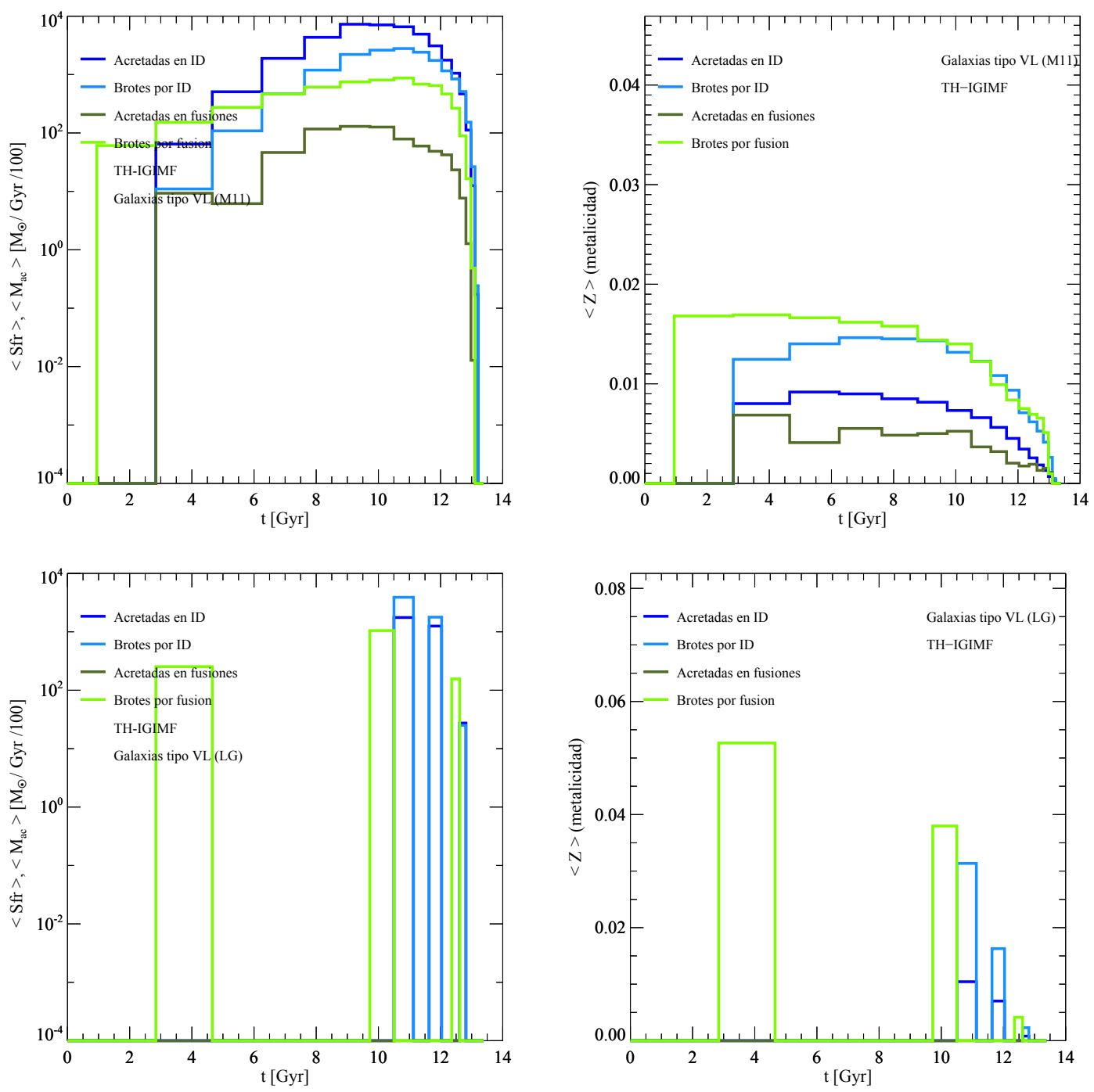

Figura 5.10: Panel superior izquierdo: Tasa de formación estelar promediada cada 5 snapshots en brotes por fusiones e inestabilidades de disco, y cantidad de masa acretada promediada cada 5 snapshots de estrellas acretadas en fusiones e inestabilidades de disco. Todas las cantidades son graficadas en función del lookback time. El código de colores está indicado en las etiquetas. Los resultados son para galaxias tipo VL generadas por el modelo con una TH-IGIMF, SAGTH5B2 y seleccionadas según el criterio de M11. Panel superior derecho: Metalicidad de las poblaciones estelares formadas, promediada cada 5 snapshots en función del lookback time. Los resultados son para galaxias tipo VL generadas por el modelo con SAGTH5B2 y seleccionadas según el criterio de M11. Paneles inferiores izquierdo y derecho: Similares a los paneles superiores izquierdo y derecho, respectivamente, pero para las galaxias tipo VL seleccionadas según el criterio de G14. 
de esas poblaciones, que superan los $10 \mathrm{Gyr}$. Las observaciones de edades de estrellas gigantes del bulbo indician que la gran mayoría de ellas son tan viejas como las PEs del modelo (Zoccali et al., 2003). El panel superior derecho muestra la evolución de la metalicidad en función del lookback-time. Se puede observar que aún hasta $8 \mathrm{Gyr}$ se forman PEs, aunque la masa de las mismas es extremadamente pequeña. La metalicidad de las PEs crece a medida que continúa la formación de sucesivas PEs, como es esperable que suceda. Los paneles inferiores izquierdo y derecho muestran los mismos resultados que los paneles superiores, pero para galaxias tipo VL seleccionadas con el criterio de pertenencia a grupos análogos al LG. Se ve que los gráficos son más ruidosos, debido al bajo número de galaxias de esta muestra. Puede verse, sin embargo, que el intervalo de edades en que se forman las PEs sigue siendo acotado, alrededor de los $10 \mathrm{Gyr}$. En la Fig. 5.10, se muestran resultados similares que en la Fig. 5.9 pero para el modelo SAGTH5B2 que utiliza la TH-IGIMF. En el panel superior izquierdo están representadas las tasas de formación estelar y de acreción de PEs originadas en brotes y en acreciones, respectivamente, de galaxias tipo VL seleccionadas según el criterio de M11. En este caso puede verse que la historia de formación de las PEs es distinta a la mostrada por las PEs en el modelo con la IMF de Salpeter y ocurre en un intervalo de tiempo prolongado, con PEs con un pico de edades en 10 Gyr y una cantidad no despreciable de PEs más jóvenes, de hasta 6 Gyr de edad, y otras tantas más viejas, de hasta 12 Gyr de edad. Aquí se observa el efecto de la retroalimentación en la historia de formación de los bulbos mencionado en la sección 4.2. En el caso de las PEs acretadas por inestabilidades de disco, la mayor proporción de estrellas masivas debido a la TH-IGIMF actúa prolongando la formación estelar en los discos, que crecen en masa a lo largo del tiempo para luego ser inestabilizados de manera que sus estrellas pasan a formar parte del bulbo. En el caso de los brotes, la retroalimentación produce una prolongación en el consumo del gas, generando la distribución amplia de la tasa de formación estelar en el tiempo. Se confirma analizando la historia de formación de las galaxias lo que ya habamos mencionado en la Sección 4.1. Las PEs estelares de los bulbos de las galaxias tipo VL seleccionadas en el modelo, por dos métodos diferentes, considerando el contexto cosmológico en un caso, y las propiedades fotométricas del bulbo de la VL en el otro, además de su masa estelar, resultan estar dominadas mayoritariamente por PEs acretadas o formadas en brotes estelares en eventos de inestabilidad del disco, de acuerdo a los resultados encontrados por Ness et al. (2012) y Ness et al. (2013) mediante el 
uso de simualciones numéricas. Sin embargo, no son despreciables los aportes de los otros orígenes posibles incluidos en el modelo, como los brotes originados en fusiones. Las acreciones de estrellas de galaxias satélites resultan mucho menos numerosas. Los diferentes modelos, que utilizan distintas IMFs, presentan algunas diferencias en la dispersión de las edades de las PEs, pero en ambos modelos las PEs tienen en promedio una edad de $10 \mathrm{Gyr}$, en concordancia con las estimaciones observacionales.

\subsection{Discusión}

En las últimas décadas, a medida que fueron publicados datos cada vez más precisos sobre las abundancias de estrellas del bulbo (ver Sección. 4.1), se realizaron esfuerzos desde el lado de la teoría para presentar modelos que pudieran explicar las características observadas de esta elusiva componente de la galaxia. Los primeros intentos en esta dirección fueron realizados por Koeppen \& Arimoto $(1989,1990)$, que suponían la caída de gas primordial en una escala de tiempo impuesta de 0,1 Gyr junto con IMFs de pendiente más plana que la de Salpeter. La rápida formación estelar se suponía frenada por la aparición de vientos de SN, logrando reproducir de manera adecuada las DMs, pero omitían considerar las SNs Ia y utilizaban la aproximación de reciclado instantáneo. Luego Matteucci \& Brocato (1990) y Matteucci \& Romano (1999) lograron relajar estas suposiciones y también propusieron IMFs más planas que Salpeter $(1,1 \lesssim x \lesssim 1,3)$. Ambos modelos suponían escalas muy cortas de formación, del orden de $0,1 \mathrm{Gyr}$ y lograban reproducir correctamente las DMs disponibles en la época. Más recientemente, Ballero et al. (2007) volvió a utilizar un modelo químico analítico para estudiar la formación del bulbo de la VL. Confirmaron con su estudio que el bulbo de la VL debería haberse formado en escalas de tiempo cortas $(\lesssim 1 \mathrm{Gyr})$ y con una alta eficiencia de formación, sumado a una pendiente de IMF plana. Resultados muy similares a los modelos anteriores, pero ahora basados en abundancias medidas con mucho mayor precisión.

Todos los modelos mencionados hasta aquí comparten la característica de no considerar efectos asociados al contexto cosmológico y de formación de estructura a gran escala. Este escenario es tenido en cuenta en el trabajo de De Lucia et al. (2014). Ellos utilizan un modelo semianalítico y lo aplican a 6 halos de MO simulados de alta resolución y formados por acreción de halos más pequeños, con masas similares a la VL, y un sistema de satélites también análogo, sin ningún vecino masivo, seleccionados especial- 
mente para tratar de reproducir las condiciones de formación del halo de la VL (Cooper et al., 2010). Ellos aplican una técnica similar a la utilizada en este trabajo para estudiar las PEs de las distintas componentes de la VL. Es interesante notar la similitud de la historia de formación estelar de los halos de MO estudiados en el trabajo mencionado (ver su Fig. 8) con la historia de formación estelar mostrada por los halos seleccionados por el criterio G14 en este capítulo (Fig. 5.5). Ellos llegan a resultados muy buenos para los discos de estas galaxias, que reproducen con mucha certeza los datos observados para esta componente, pero muestran resultados peor comportados para los esferoides, que sólo consideran que se forman por acreción de satélites, la componente que menos aporta a la formación de bulbos en nuestro caso. Otra característica que comparten es la necesidad de incluir una IMF con pendiente más plana que Salpeter. Parece una condición inevitable en los modelos químicos de formación de bulbo la existencia de una IMF de este tipo para lograr una contaminación química aceptable, lo cual es corroborado por las conclusiones que emergen de este trabajo de tesis.

Los modelos químicos analíticos descriptos, a excepción del de De Lucia et al. (2014) parten de la suposición de que la formación del bulbo se produjo por el colapso de gas frío en una etapa temprana del Universo. La existencia de un gradiente de metalicidades en la dirección del eje vertical del bulbo junto con un alto nivel de enriquecimiento de elementos $\alpha$ en estrellas son comúnmente considerados en combinación como un proxy de formación rápida por colapso disipativo de gas. Sin embargo, en un trabajo reciente de Ness et al. (2013) del relevamiento ARGOS, se encuentra que el gradiente de metalicidades puede ser explicado considerando dos poblaciones provenientes de eventos de inestabilidad de disco, que tienen distintas abundancias a diferentes latitudes. Esta opción está en línea con el hallazgo de que el bulbo tiene una forma de X, consistente con los bulbos mani/boxy, que se creen originados en eventos de inestabilidad. Esto fue confirmado recientemente midiendo la cinemática de las estrellas del bulbo (Vásquez et al., 2013), que observan las velocidades radiales en los brazos de la forma de X predicha para el bulbo. Los resultados encontrados en nuestro trabajo apoyan una interpretación sobre la formación del bulbo de la VL en la que la mayor parte de las estrellas proviene del disco temprano de la VL. 



\section{Capítulo 6}

\section{Conclusiones}

When I heard the learnd astronomer;

When the proofs, the figures, were ranged in columns

before me;

When I was shown the charts and the diagrams, to add,

divide, and measure them;

When I, sitting, heard the astronomer, where he lectured with much applause in the lecture-room, How soon, unaccountable, I became tired and sick;

Till rising and gliding out, I wanderd off by myself, In the mystical moist night-air, and from time to time, Lookd up in perfect silence at the stars.

Walt Whitman - Leaves of grass (1885)

Este tabajo de tesis es un trabajo de investigación teórico que contribuye al conocimiento en el área de formación y evolución de galaxias. El mismo ha sido desarrollado mediante el uso de técnicas numéricas. Más específicamente, utilizamos un modelo de formación y evolución de galaxias que combina una simulación cosmológica sin disipación de $N$-cuerpos con el modelo semianalítico de formación y evolución de galaxias SAG (Cora, 2006; Lagos et al., 2008a; Tecce et al., 2010) para estudiar las propiedades químicas de las componentes estelares de galaxias elípticas y de las poblaciones estelares de los bulbos de galaxias tipo VL. En particular, estudiamos los aspectos físicos detrás de la construcción de la relación $[\alpha / \mathrm{Fe}]$-masa estelar característica de galaxias 
elípticas, conocido como downsizing quemo-arqueológico (Fontanot et al., 2009). Por un lado, nos interesaba el problema en sí mismo, por su vigencia y la importancia en el marco del paradigma jerárquico de formación de galaxias y de estructura a gran escala del Universo. Por otro lado, resulta de interés porque ayuda a restringir el modelado de los procesos físicos que impactan en la evolución química de las componentes bariónicas de las galaxias generadas por el modelo semianalítico SAG, y más precisamente, de la componente esferoidal que constituye la componente estelar dominante de galaxias elípticas la cual se encuentra afectada por mismos procesos de formación que esta clase de galaxias. Nuestro análisis del bulbo estelar nos permite enfocarnos además en los bulbos de las galaxias tipo Vía Láctea (VL). La suposición subyacente a ambos aspectos involucrados en este trabajo es que las propiedades químicas de las poblaciones estelares de las galaxias elípticas y de los bulbos de las galaxias con condiciones similares a la VL no pueden ser explicadas aplicando una distribución estelar inicial de masas simple y universal, sino que nuevas consideraciones deben ser tenidas en cuenta. Los modelos modernos de formación y evolución de galaxias deben considerar la complejidad asociada a este aspecto de la formación estelar. Presentamos aquí las conclusiones específicas obtenidas en el desarrollo de las diferentes etapas del trabajo de tesis, detallando las conclusiones obtenidas en el desarrollo de los Capítulo 4 y 5.

Nos enfocamos primero en la relación $[\alpha / \mathrm{Fe}]$-masa estelar de galaxias elípticas. Una versión del modelo semianalítico SAG que involucra una IMF universal como la IMF de Salpeter no es capaz de reproducir la pendiente correcta de la relación $[\alpha / \mathrm{Fe}]-$ masa estelar una vez que el modelado de varios aspectos de SAG han sido mejorados (como las escalas de tiempo de brotes estelares).

Intentos tempranos de modelar la abundancia de $[\alpha / \mathrm{Fe}]$ descriptos en la literatura han fallado en reproducir la relación observada con la masa estelar, salvo cuando se invocan hipótesis ad hoc, como un cambio en la pendiente de la IMF global (Arrigoni et al., 2010), una IMF arbitraria dependiente de la SFR (Calura \& Menci, 2009), brotes en las galaxias progenitoras disparados por encuentros cercanos (Calura \& Menci, 2011) o tiempos de retardo de SNs Ia adoptados empíricamente (Yates et al., 2013). Motivados por el hecho de que una IMF universal no permite reproducir la tendencia observada entre el cociente de abundancias $[\alpha / \mathrm{Fe}]$ y su masa estelar en galaxias elípticas, exploramos el impacto de una IMF variable en su relación $[\alpha / \mathrm{Fe}]$-masa estelar.

Logramos cocientes de $[\alpha / \mathrm{Fe}]$ para diferentes masas estelares consistentes con los 
observados, implementando en nuestro modelo semianalítico una IMF dependiente de la SFR que resulta de una teoría físicamente motivada (TH-IGIMF, WKP11). La consistencia de esta implementación y el hecho de que muchas otras propiedades observadas de galaxias y sus relaciones son simultáneamente reproducidas, hacen más robustas nuestras conclusiones. Encontramos que el uso de una TH-IGIMF para representar la IMF de las galaxias como un todo, es necesaria para un tratamiento acorde del enriquecimiento químico de las galaxias. Resumimos los principales resultados específicos de este aspecto de nuestro trabajo:

- Un modelo de formación de galaxias con una TH-IGIMF puede recobrar la pendiente positiva de la relación $[\alpha / \mathrm{Fe}]$-masa estelar observada (Fig. 4.3). Este es el caso para todos los valores considerados para la masa mínima de cúmulo inmerso $M_{\text {ecl }}^{\min }=5$ y $100 \mathrm{M}_{\odot}$ y para la pendiente de la función de masa de cúmulos inmersos $\beta=2$ y 2,1 , los cuales son parámetros libres involucrados en la teoría de TH-IGIMF. Sin embargo, el acuerdo con la tendencia observada es particularmente bueno para $M_{\mathrm{ecl}}^{\min }=5 \mathrm{M}_{\odot}$ y $\beta=2$. Así, nuestros resultados favorecen un valor pequeño para la masa mínima de cúmulo embebido, y están de acuerdo con el valor estándar de la pendiente de la función de cúmulos inmersos, $\beta_{2}$.

- Sin importar el valor de los parámetros libres de la TH-IGIMF, la pendiente media de la TH-IGIMF $\left(\left\langle\alpha_{\mathrm{TH}}\right\rangle\right)$ se vuelve progresivamente menor para galaxias más masivas (Fig. 4.4). Ya que esta pendiente es dependiente de la SFR, este comportamiento es una manifestación del hecho de que galaxias más masivas tienen SFRs más altas. La diferencia relativa de la pendiente de la IMF para galaxias con diferentes masas es clave para reproducir la relación $[\alpha / \mathrm{Fe}]$-masa estelar. Esto es demostrado por nuestro mejor modelo, caracterizado por una $M_{\mathrm{ecl}}^{\min }=5 \mathrm{M}_{\odot} \mathrm{y}$ $\beta=2$. En este caso, la TH-IGIMF es más empinada que la IMF de Salpeter para los intervalos de masa más chicos y más plana para los intervalos de masa mayores, mostrando que no es una condición necesaria una IMF top-heavy para todos los intervalos de masa para la construcción de la relación $[\alpha / \mathrm{Fe}]$-masa estelar como se cree generalmente. Nuestros resultados indican que galaxias con masas menores a $\sim 3 \times 10^{10} \mathrm{M}_{\odot}$ deberían tener una IMF más empinada que la IMF de Salpeter, mientras que galaxias con mayores masas deberían tener una IMF más plana que la IMF de Salpeter para lograr tener las abundancias correctas. 
- Una tendencia de downsizing leve es evidente en las galaxias generadas por los modelos con diferente IMF. Las distribuciones de redshifts de formación $\left(z_{\text {form }(50)}\right)$ y de escalas de tiempo de formación $\left(\Delta T_{50}\right)$ de galaxias elípticas generadas por los modelos SAGTH5B2 y SAGS, caracterizados por una TH-IGIMF $\operatorname{con} M_{\mathrm{ecl}}^{\min }=$ $5 \mathrm{M}_{\odot}$ y $\beta=2$ y la IMF de Salpeter, respectivamente, tienen características y dependencias con la masa estelar similares (Figs. 4.12 y 4.13 ) apoyando el hecho de que la elección de la IMF tiene un efecto despreciable en las escalas de tiempo de formación. En ambos casos, las galaxias menos masivas $\left(M_{\star}<10^{9} \mathrm{M}_{\odot}\right)$ logran el 50 por ciento de sus masas estelares formadas in-situ a menores redshifts que las más masivas, con la mayoría de ellas formándose entre $z \approx 0$ y $z \approx 0,5$. El pico de las distribuciones para galaxias más masivas que $\approx 10^{9} \mathrm{M}_{\odot}$ se corre desde $0 \lesssim z_{\text {form }(50)} \lesssim 0,5$ a $0,5 \lesssim z_{\text {form(50) }} \lesssim 1$. Las distribuciones de $\Delta T_{50}$ son muy similares para galaxias de diferente masa, mostrando valores correspondientes a la muestra completa de galaxias $(\approx 2-2,5 \mathrm{Gyr})$. Esta tendencia de downsizing leve presente en los modelos tanto con una IMF universal como una IMF variable indica que las escales de tiempo de formación de las galaxias no juegan un rol decisivo en determinar la pendiente de la relación $[\alpha / \mathrm{Fe}]-$ masa estelar, las cuales son claramente diferentes cuando se usa una TH-IGIMF o la IMF de Salpeter. Debe ser tenido en cuenta, sin embargo, que esta tendencia leve es una característica de los modelos semianalíticos. Si a la escala de tiempo de formación estelar se le permitiera variar más libremente, entonces también contribuiría a producir una dependencia de las abundancias $[\alpha / \mathrm{Fe}]$ con la masa estelar. Sin embargo, en este caso, otras propiedades de la población de galaxias en un contexto cosmológico podrían no comportarse apropiadamente. Usando un modelo semianalítico somos capaces de ajustar la relación $[\alpha / \mathrm{Fe}]$-masa estelar al mismo tiempo que la función de luminosidad produce resultados concordantes con las observaciones. Este requerimiento agrega limitaciones adicionales a los SAMs, los cuales mostramos que aún así pueden proveer una relación $[\alpha / \mathrm{Fe}]$-masa estelar para galaxias elípticas razonable. Nuestra conclusión no descarta la influencia del downsizing en el desarrollo de esta relación; es sólo que este efecto no está manifestado fuertemente en los modelos de formación galáctica.

- Las galaxias más viejas (aquéllas con $z_{\text {form(50) }}$ más altos) son formadas en es- 
calas de tiempo de formación más cortas; las galaxias que se han formado a $z_{\text {form(50) }} \approx 2$, o antes, tienen $\Delta \mathrm{T}_{50} \lesssim 2 \mathrm{Gyr}$ (Fig. 4.11). Por otro lado, las galaxias con escalas de tiempo de formación más cortas tienen valores mayores de cocientes de abundancia $[\alpha / \mathrm{Fe}]$ (Fig. 4.14). Entonces, las galaxias más viejas logran mayores valores de $[\alpha / \mathrm{Fe}]$ en acuerdo con datos observacionales (SánchezBlázquez et al., 2006; Trager et al., 2000). Esta correlación está presente para galaxias de diferente masa, explicando así la dispersión de la relación $[\alpha / \mathrm{Fe}]-m a s a$ estelar para una dada masa.

- El cociente $M / L$ de galaxias masivas tiene formas similares en ambos modelos SAGTH5B2 y SAGS, mostrando un exceso para galaxias de baja masa $(\lesssim$ $2 \times 10^{10} \mathrm{M}_{\odot}$ ) respecto a las observaciones (Fig. 4.9). Sin embargo, el modelo SAGTH5B2 permite lograr los valores correctos de cocientes $M / L$ para galaxias masivas. Esto conduce a un mejor acuerdo con los datos observacionales. El extremo brillante de la función de luminosidad en la banda- $r$ también se comporta mejor en el modelo SAGTH5B2 (Fig. 4.10).

A partir de la interpretación de los datos observacionales (e.g. Thomas et al., 2005), una explicación generalmente aceptada para la construcción de la relación $[\alpha / \mathrm{Fe}]$-masa estelar ha sido las escalas de tiempo de formación relativamente cortas de galaxias masivas. Basados en este argumento de escalas de tiempo, deberíamos obtener valores de $[\alpha / \mathrm{Fe}]$ más altos para estas galaxias, ya que tienen menores posiblidades de formar estrellas del gas frío contaminado por los productos de $\mathrm{SNs}$ Ia. El análisis detallado realizado en este trabajo de tesis tuvo el propósito de determinar si las escalas de tiempo de formación son responsables de la pendiente positiva en la relación $[\alpha / \mathrm{Fe}]$-masa estelar. De esta investigación, podemos concluir que este argumento sólo permite explicar la dispersión de valores de $[\alpha / \mathrm{Fe}]$ para galaxias de una dada masa, pero no puede ser el responsable de la construcción de la dependencia con la masa. Nuestros resultados, basados en un modelo de formación de galaxias jerárquico, muestra que la presencia de una TH-IGIMF dependiente de la SFR es una explicación válida para la existencia de esta relación entre los cocientes de abundancia de $[\alpha / \mathrm{Fe}]$ y la masa estelar. Una IMF cuya pendiente depende de la SFR da diferentes números relativos de progenitores de SNs Ia y SNs II para cada evento de formación estelar, el cual es un aspecto clave en la construcción de la relación estudiada. Las galaxias masivas alcanzan cocientes de 
abundancias de $[\alpha / \mathrm{Fe}]$ más altos porque están caracterizados por IMFs integradas más planas, como resultado de sus SFRs más altas.

Los resultados obtenidos para las propiedades químicas de galaxias elípticas validan el uso de nuestro modelo semianalítico para profundizar el análisis de las propiedades de la componente esferoidal de las galaxias, que constituye como mínimo un 80 por ciento de la población estelar total de las elípticas. En particular, enfocamos nuestro estudio en los bulbos de galaxias con características equivalentes a la VL, las cuales fueron seleccionadas considerando dos conjuntos de criterios diferentes, uno que incluye propiedades fotométricas de las galaxias (M11) y el otro que considera la pertenencia de las galaxias a sistemas análogos al Grupo Local (LG; G14). Evaluamos el impacto de una IMF de Salteter y de la TH-IGIMF caracterizada por $M_{\mathrm{ecl}}^{\min }=5 \mathrm{M}_{\odot}$ y $\beta=2$ (a la que nos referimos simplemente como TH-IGIMF de aquí en más) en las propiedades químicas de las poblaciones estelares (PEs) de las galaxias en estas dos muestras, obteniendo las siguientes conclusiones:

- El modelo SAGTH5B2, caracterizado por la TH-IGIMF, presenta una mayor cantidad de galaxias tipo VL seleccionadas por el criterio M11 que el modelo SAGS. El número de galaxias tipo VL seleccionadas por el criterio de pertenencia a grupos análogos al LG es el mismo en los dos casos, ya que los halos de materia oscura se seleccionan sobre la simulación subyacente. Los criterios de selección aplicados a las poblaciones obtenidas de ambos modelos coinciden para un mayor número de galaxias en el caso del modelo SAGTH5B2. Los bulbos de las galaxias tipo VL seleccionadas por el criterio de M11 tienen masas estelares restringidas por el cociente de luminosidades requerido por el mismo. En el caso del modelo SAGS, las masas estelares de los bulbos de las galaxias tipo VL seleccionadas por este criterio son menores, en un orden de magnitud, en promedio, que las masas dinámicas obtenidas a partir de observaciones de la cinemática de las estrellas del bulbo. En el modelo SAGTH5B2, las masas estelares de los bulbos de galaxias seleccionadas con el criterio de M11 están en acuerdo con las masas estimadas observacionalmente en promedio. Los bulbos de galaxias tipo VL seleccionadas por el criterio de grupos análogos a LG tienen masas estelares en un intervalo muy amplio de masas para los dos modelos, pero existe un mayor número de bulbos con masas en acuerdo a las observaciones para el modelo SAGTH5B2. 
- Un modelo de formación de galaxias con una TH-IGIMF puede reproducir de mejor manera la distribución de metalicidades de estrellas del bulbo de la VL, derivadas a partir de observaciones espectroscópicas que el mismo modelo utilizando una IMF de Salpeter (Fig. 5.3 y Fig. 5.4). Esto se cumple para las galaxias tipo VL identificadas por los dos criterios considerados, el de M11 y el G14. Este hecho resulta más evidente para las galaxias tipo VL seleccionadas mediante el criterio de M11, cuyas PEs obtenidas del modelo con IMF de Salpeter, SAGS, presentan una distribución de metalicidades con un valor medio muy lejano y menor al calculado a partir de mediciones observacionales.

- Las historias de formación estelar de las galaxias tipo VL del modelo SAGTH5B2 seleccionadas por los dos criterios considerados en este trabajo presentan diferencias notables (Fig. 5.5, que determinan la adopción de diferentes pendientes de su IMF, y por lo tanto, determinan la historia de enriquecimiento químico de sus PEs $\mathrm{y}$, en particular, de las PEs de su bulbo. Las galaxias tipo VL seleccionadas de acuerdo al criterio de pertenencia a los grupos análogos al LG tienen un pico de formación estelar en épocas tempranas (lookback time $\approx 10 \mathrm{Gyr}$ ) y bajos niveles de formación posterior lo cual determina la formación de PEs del bulbo viejas con una distribución de edades sin dispersión (Fig. 5.10). El pico de formación estelar alcanza niveles muy altos y produce un gran enriquecimiento del gas de la galaxia debido a las pendientes planas alcanzadas por la IMF. Las características de la formación estelar de las galaxias tipo VL en el modelo SAGTH5B2 seleccionadas según este criterio que involucra el origen cosmológico de las galaxias se refleja en una distribución de metalicidades picuda y corrida hacia altas metalicidades con un valor medio de metalicidad muy cercano al observado (panel inferior izquierdo, Fig. 5.4). La historia de formación de las galaxias tipo VL seleccionadas con el criterio de M11 en el modelo SAGTH5B2 muestra una formación estelar prolongada en el tiempo, con pendientes $\alpha_{3}$ con valores cercanos al valor de la pendiente de la IMF de Salpeter, lo cual significa una mayor cantidad de estrellas masivas en la distribución, por efectos de la normalización de la TH-IGIMF. Esto se refleja en una distribución de metalicidades con un valor medio cercano al observado y con una dispersión de metalicidades similar a la observada (panel superior izquierdo, Fig. 5.4). 
- El análisis de las proporciones de PEs formadas por distintos mecanismos de formación de los bulbos de galaxias tipo VL lleva a la conclusión inequívoca de que los bulbos de este tipo de galaxias están formados por PEs originadas en eventos de inestabilidad de disco. Más aún, los resultados mostrados por los modelos SAGS y SAGTH5B2 para las galaxia tipo VL seleccionadas por el criterio de M11 y de G14 evidencian que, además, la mayor proporción de PEs son acretadas directamente del disco y en menor medida formadas en brotes originados en estos eventos de inestabilidad, en acuerdo con los últimos resultados de la cinemática de las estrellas del bulbo, que indican que el mismo estaría formado casi exclusivamente por procesos seculares. Las PEs acretadas en fusiones no representan una fracción considerable en ninguno de los casos estudiados, aunque los brotes generados por fusiones son más frecuentes. Del análisis detallado de las PEs formadas por diferentes orígenes se desprende que los brotes por fusiones forman PEs pobres en metales, mientras que las PEs acretadas en inestabilidades de disco tienen abundancias de hierro entre medias y altas. Los brotes producidos en eventos de inestabilidad presentan las metalicidades más altas.

- El análisis de los resultados de la aplicación del método fotométrico de obtención de metalicidades a las salidas del programa IAC-STAR, cuyos datos de entrada son los resultados provistos por el modelo semianalítico, arroja conclusiones que ponen en discusión el alcance de este método observacional para medir metalicidades. El método sólo pudo ser aplicado al modelo SAGS, ya que el programa IAC-STAR no cuenta con opciones para IMFs variables. Se encontró que los valores medios de las distribuciones de metalicidad calculadas mediante este método son similares a las metalicidades derivadas directamente del modelo cuando se consideran las galaxias tipo VL seleccionadas por el criterio de M11, mientras que el valor medio se ve reducido en aproximadamente 1 dex respecto a las metalicidades del modelo cuando se aplica el criterio G14 para identificar galaxias tipo VL (Fig. 5.7). La correcta interpretación de esta inconsistencia demandará un análisis más detallado de las suposiones involucradas en este método y las limitaciones de los programas utilizados.

- Los diagramas de abundancias de elementos $\alpha$ respecto al hierro, en función de las abundancias $[\mathrm{Fe} / \mathrm{H}]$ de las PEs de los modelos SAGS y SAGTH5B2 reflejan 
las características mencionadas anteriormente. Existe un mayor número de PEs de galaxias VL seleccionadas con el criterio de M11 para el modelo SAGTH5B2, producto del mayor número de galaxias tipo VL dentro del intervalo de selección. Existe un desfasaje mayor en el eje de $[\mathrm{Fe} / \mathrm{H}]$ para el modelo SAGS respecto a las observaciones, producto de la menor contaminación química exhibida en las distribuciones de metalicidades. Sin embargo, una característica resulta común a las PEs de los dos modelos. La pendiente de decrecimiento de $[\alpha / \mathrm{Fe}]$ a medida que crece el cociente de abundancias $[\mathrm{Fe} / \mathrm{H}]$ mostrada por las PEs, es similar en los modelos con diferente IMF y en todos los posibles orígenes de las PEs, siendo una pendiente más negativa que la mostrada por las observaciones (Fig. 5.8). Esto indica que el problema no proviene de las escalas de tiempo de formación consideradas en los mecanismos de formación. Se especula sobre la posibilidad de que la razón de esta discrepancia con las observaciones radique en las distribuciones de tiempo de retardo de las SNs Ia, responsables de eyección del hierro al medio intergaláctico.

Los resultados y conclusiones alcanzados a través de la técnica numérica aplicada, consistente en la combinación de simulaciones numéricas y un modelo semianalítico de formación y evolución de galaxias, junto con la implementación del seguimiento de poblaciones estelares en este último, representan un primer intento hacia el entendimiento de los procesos físicos involucrados en la formación y evolución de los esferoides, entendidos como sistemas estelares complejos y formados por múltiples poblaciones de estrellas con distinto origen, como son las galaxias elípticas y los bulbos de las galaxias. Este trabajo de investigación plantea numerosos interrogantes sobre las propiedades químicas de los esferoides. Es necesario investigar en profundidad el impacto de la aplicación de diferentes recetas de tiempos de retardo de SNs Ia y de la ya mencionada incorporación de elementos pesados en el modelo químico (Ver Discusión en Capítulo 4.) El estudio de abundancias químicas de elementos individuales puede ayudar a dirimir las diferentes suposiciones realizadas tanto por observadores como por teóricos.

La experiencia recolectada y las técnicas implementadas sientan las bases para llevar a cabo el estudio detallado de las propiedades de las demás componentes de la Vía Láctea. El estudio de la formación y evolución del halo de la Vía Láctea es un camino natural a seguir que se abre a partir de este trabajo de investigación y de una versión 
actualizada del modelo semianalítico que tiene en cuenta procesos de ambiente, tal como la disrupción por mareas que da lugar a la formación del halo estelar (Cora et al., en preparación). 


\section{Bibliografía}

Abadi M. G., Navarro J. F., Fardal M., Babul A., Steinmetz M., 2010, Mon. Not. R. Astron. Soc. , 407, 435

Adelman2006 2006, ApJS, 162, 38

Alves-Brito A., Meléndez J., Asplund M., Ramírez I., Yong D., 2010, Astron. Astrophys. , 513, A35

Anders E., Grevesse N., 1989, Geochimica Cosmochimica Acta, 53, 197

Aparicio A., Gallart C., 2004, AJ, 128, 1465

Arrigoni M., Trager S. C., Somerville R. S., Gibson B. K., 2010, MNRAS, 402, 173

Athanassoula E., 2013, Bars and secular evolution in disk galaxies: Theoretical input. p. 305

Auger M. W., Treu T., Gavazzi R., Bolton A. S., Koopmans L. V. E., Marshall P. J., 2010, ApJL, 721, L163

Babcock H. W., 1939, Lick Observatory Bulletin, 19, 41

Ballero S. K., Matteucci F., Origlia L., Rich R. M., 2007, Astron. Astrophys. , 467, 123

Balogh M. L., Navarro J. F., Morris S. L., 2000, Astrophys. J. , 540, 113

Baugh C. M., Lacey C. G., Frenk C. S., Granato G. L., Silva L., Bressan A., Benson A. J., Cole S., 2005, Mon. Not. R. Astron. Soc. , 356, 1191

Bensby T., Adén D., Meléndez J., et al. 2011, Astron. Astrophys. , 533, A134

Bensby T., Feltzing S., Lundström I., 2004, Astron. Astrophys. , 415, 155

Bensby T., Yee J. C., Feltzing e. a., 2013, Astron. Astrophys. , 549, A147

Benson A. J., Borgani S., De Lucia G., Boylan-Kolchin M., Monaco P., 2012, MNRAS, 419, 3590

Bernardi M., Nichol R. C., Sheth R. K., Miller C. J., Brinkmann J., 2006, AJ, 131, 1288 
Bertschinger E., 2001, ApJS, 137, 1

Bessell M. S., 1990, PASP, 102, 1181

Blanton M. R., Lupton R. H., Schlegel D. J., Strauss M. A., Brinkmann J., Fukugita M., Loveday J., 2005, ApJ, 631, 208

Blumenthal G. R., Faber S. M., Flores R., Primack J. R., 1986, Astrophys. J. , 301, 27

Bond J. R., Cole S., Efstathiou G., Kaiser N., 1991, Astrophys. J. , 379, 440

Bond J. R., Szalay A. S., 1983, Astrophys. J. , 274, 443

Botticella M. T., Smartt S. J., Kennicutt R. C., Cappellaro E., Sereno M., Lee J. C., 2012, A\&A, 537, A132

Boylan-Kolchin M., Springel V., White S. D. M., Jenkins A., Lemson G., 2009, Mon. Not. R. Astron. Soc. , 398, 1150

Bruzual G., 2007, in Vallenari A., Tantalo R., Portinari L., Moretti A., eds, From Stars to Galaxies: Building the Pieces to Build Up the Universe Vol. 374 of Astronomical Society of the Pacific Conference Series, Stellar Populations: High Spectral Resolution Libraries. Improved TP-AGB Treatment. p. 303

Bruzual G., Charlot S., 2003, MNRAS, 344, 1000

Bryan G. L., Norman M. L., 1998, Astrophys. J. , 495, 80

Bullock J. S., Kolatt T. S., Sigad Y., Somerville R. S., Kravtsov A. V., Klypin A. A., Primack J. R., Dekel A., 2001, Mon. Not. R. Astron. Soc. , 321, 559

Calura F., Menci N., 2009, MNRAS, 400, 1347

Calura F., Menci N., 2011, MNRAS, 413, L1

Cappellari M., Bacon R., Bureau M., Damen M. C., Davies R. L., de Zeeuw P. T., Emsellem E., FalcónBarroso J., Krajnović D., Kuntschner H., McDermid R. M., Peletier R. F., Sarzi M., van den Bosch R. C. E., van de Ven G., 2006, MNRAS, 366, 1126

Cappellari M., Emsellem E., Krajnović D., McDermid R. M., et al. 2011, MNRAS, 413, 813

Cappellari M., McDermid R. M., Alatalo K., Blitz L., Bois M., Bournaud F., Bureau M., Crocker A. F., et al. 2012, Nature, 484, 485

Cappellari M., McDermid R. M., Alatalo K., Blitz L., et al. 2013, MNRAS, 432, 1862

Cappellari M., Scott N., Alatalo K., Blitz L., et al. 2013, MNRAS, 432, 1709 
Cappellaro E., Evans R., Turatto M., 1999, A\&A, 351, 459

Cardelli J. A., Clayton G. C., Mathis J. S., 1989, Astrophys. J. , 345, 245

Carr B. J., Arnett W. D., Bond J. R., 1982, in Rees M. J., Stoneham R. J., eds, NATO Advanced Science Institutes (ASI) Series C Vol. 90 of NATO Advanced Science Institutes (ASI) Series C, Pregalactic very massive objects and their cosmological consequences. pp 313-318

Carroll S. M., Press W. H., Turner E. L., 1992, ARA\&A, 30, 499

Cavaliere A., Vittorini V., 2000, ApJ, 543, 599

Cenarro A. J., Gorgas J., Vazdekis A., Cardiel N., Peletier R. F., 2003, MNRAS, 339, L12

Cenarro A. J., Sánchez-Blázquez P., Cardiel N., Gorgas J., 2004, ApJL, 614, L101

Chabrier G., 2003, pasp, 115, 763

Chiosi C., Bressan A., Portinari L., Tantalo R., 1998, A\&A, 339, 355

Cole S., 1991, Astrophys. J. , 367, 45

Colless M., Dunn A. M., 1996, Astrophys. J. , 458, 435

Conroy C., Dutton A. A., Graves G. J., Mendel J. T., van Dokkum P. G., 2013, ApJL, 776, L26

Conroy C., van Dokkum P. G., Graves G. J., 2013, ApJL, 763, L25

Conselice C. J., 2006, MNRAS, 373, 1389

Cooper A. P., Cole S., Frenk C. S., White S. D. M., Helly J., Benson A. J., De Lucia G., Helmi A., Jenkins A., Navarro J. F., Springel V., Wang J., 2010, Mon. Not. R. Astron. Soc. , 406, 744

Cora S. A., 2006, MNRAS, 368, 1540

Correa C. A., Wyithe J. S. B., Schaye J., Duffy A. R., 2014, ArXiv e-prints

Cowie L. L., Barger A. J., 2008, ApJ, 686, 72

Cowie L. L., Songaila A., Hu E. M., Cohen J. G., 1996, AJ, 112, 839

Croton D. J., Springel V., White S. D. M., De Lucia G., Frenk C. S., Gao L., Jenkins A., Kauffmann G., Navarro J. F., Yoshida N., 2006, MNRAS, 365, 11

De Lucia G., Springel V., White S. D. M., Croton D., Kauffmann G., 2006, MNRAS, 366, 499 
De Lucia G., Tornatore L., Frenk C. S., Helmi A., Navarro J. F., White S. D. M., 2014, Mon. Not. R. Astron. Soc. , 445, 970

de Vaucouleurs G., de Vaucouleurs A., Corwin Jr. H. G., 1976, Second reference catalogue of bright galaxies. Containing information on 4,364 galaxies with references to papers published between 1964 and 1975.

Di Matteo P., Bournaud F., Martig M., Combes F., Melchior A.-L., Semelin B., 2008, A\&A, 492, 31

Dubinski J., Carlberg R. G., 1991, Astrophys. J. , 378, 496

Dunkley J., Komatsu E., Nolta M. R., Spergel D.Ñ., Larson D., Hinshaw G., Page L., Bennett C. L., Gold B., et al. 2009, ApJS, 180, 306

Dutton A. A., Mendel J. T., Simard L., 2012, MNRAS, 422, L33

Efstathiou G., Lake G., Negroponte J., 1982, MNRAS, 199, 1069

Einasto J., 2010, in Ruffini R., Vereshchagin G., eds, American Institute of Physics Conference Series Vol. 1205 of American Institute of Physics Conference Series, Large scale structure of the Universe. pp 72-81

Einasto J., Saar E., Kaasik A., Chernin A. D., 1974, Nature , 252, 111

Elmegreen B. G., 2004, MNRAS, 354, 367

Faber S. M., 1973, ApJ, 179, 731

Feltzing S., Gilmore G., 2000, Astron. Astrophys. , 355, 949

Ferreras I., La Barbera F., de la Rosa I. G., Vazdekis A., de Carvalho R. R., Falcón-Barroso J., Ricciardelli E., 2013, MNRAS, 429, L15

Feulner G., Gabasch A., Salvato M., Drory N., Hopp U., Bender R., 2005, ApJL, 633, L9

Fontanot F., De Lucia G., Monaco P., Somerville R. S., Santini P., 2009, MNRAS, 397, 1776

Foster A. R., Ji L., Smith R. K., Brickhouse N. S., 2012, ApJ, 756, 128

Freeman K., Bland-Hawthorn J., 2002, A\&AAR, 40, 487

Freeman K., Ness M., Wylie-de-Boer E., Athanassoula E., Bland-Hawthorn J., Asplund M., Lewis G., Yong D., Lane R., Kiss L., Ibata R., 2013, Mon. Not. R. Astron. Soc. , 428, 3660

Fukugita M., Ichikawa T., Gunn J. E., Doi M., Shimasaku K., Schneider D. P., 1996, Astron. J. , 111, 1748 
Fulbright J. P., McWilliam A., Rich R. M., 2006, Astrophys. J. , 636, 821

Gadotti D. A., 2009, Mon. Not. R. Astron. Soc. , 393, 1531

Gallazzi A., Charlot S., Brinchmann J., White S. D. M., Tremonti C. A., 2005, MNRAS, 362, 41

Gargiulo I. D., Cora S. A., Padilla N. D., Muñoz Arancibia A. M., Ruiz A.Ñ., Orsi A. A., Tecce T. E., Weidner C., Bruzual G., 2014, ArXiv e-prints

Geisler D., Wallerstein G., Smith V. V., Casetti-Dinescu D. I., 2007, pasp, 119, 939

Girardi L., Bertelli G., Bressan A., Chiosi C., Groenewegen M. A. T., Marigo P., Salasnich B., Weiss A., 2002, Astron. Astrophys. , 391, 195

Gonzalez O. A., Rejkuba M., Zoccali M., Hill V., Battaglia G., Babusiaux C., Minniti D., Barbuy B., Alves-Brito A., Renzini A., Gomez A., Ortolani S., 2011, Astron. Astrophys. , 530, A54

Gonzalez O. A., Rejkuba M., Zoccali M., Valent E., Minniti D., Tobar R., 2013, A\&A, 552, A110

González R. E., Kravtsov A. V., Gnedin N. Y., 2013, Astrophys. J. , 770, 96

González R. E., Kravtsov A. V., Gnedin N. Y., 2014, Astrophys. J. , 793, 91

Granato G. L., Lacey C. G., Silva L., Bressan A., Baugh C. M., Cole S., Frenk C. S., 2000, ApJ, 542, 710

Greggio L., 2005, A\&A, 441, 1055

Greggio L., Renzini A., 1983, A\&A, 118, 217

Grevesse N., Noels A., Sauval A. J., 1996, in Holt S. S., Sonneborn G., eds, Cosmic Abundances Vol. 99 of Astronomical Society of the Pacific Conference Series, Standard Abundances. p. 117

Groenewegen M. A. T., Blommaert J. A. D. L., 2005, Astron. Astrophys. , 443, 143

Gunn J. E., Tinsley B. M., 1975, Nature , 257, 454

Guo Q., Cole S., Eke V., Frenk C., 2011, Mon. Not. R. Astron. Soc. , 417, 370

Guo Q., White S., Angulo R. E., Henriques B., Lemson G., Boylan-Kolchin M., Thomas P., Short C., 2013, Mon. Not. R. Astron. Soc. , 428, 1351

Häring N., Rix H.-W., 2004, ApJL, 604, L89

Hayashi E., White S. D. M., 2008, Mon. Not. R. Astron. Soc. , 388, 2

Hirschi R., Meynet G., Maeder A., 2005, A\&A, 433, 1013 
Hogg D. W., 1999, ArXiv Astrophysics e-prints

Hubble E., 1929, Contributions from the Mount Wilson Observatory, vol. 3, pp.23-28, 3, 23

Hubble E., Humason M. L., 1931, Astrophys. J. , 74, 43

Hubble E. P., 1925, The Observatory, 48, 139

Iwamoto K., Brachwitz F., Namoto K., Kishimoto N., Umeda H., Hix W. R., Thielemann F., 1999, ApJS, 125,439

Jarosik N., Bennett C. L., Dunkley J., Gold B., Greason M. R., Halpern M., Hill R. S., Hinshaw G., et al. 2011, ApJS, 192, 14

Jiménez N., Cora S. A., Bassino L. P., Tecce T. E., Smith Castelli A. V., 2011, MNRAS, 417, 785

Jing Y. P., 2000, Astrophys. J. , 535, 30

Joeveer M., Einasto J., 1978, in Longair M. S., Einasto J., eds, Large Scale Structures in the Universe Vol. 79 of IAU Symposium, Has the universe the cell structure. pp 241-250

Kahn F. D., Woltjer L., 1959, Astrophys. J. , 130, 705

Karakas A. I., 2010, MNRAS, 403, 1413

Kauffmann G., Colberg J. M., Diaferio A., White S. D. M., 1999, Mon. Not. R. Astron. Soc. , 307, 529

Kent S. M., 1992, Astrophys. J. , 387, 181

Kimm T., Yi S. K., Khochfar S., 2011, ApJ, 729, 11

Kirk H., Myers P. C., 2011, ApJ, 727, 64

Klypin A. A., Trujillo-Gomez S., Primack J., 2011, Astrophys. J. , 740, 102

Kobayashi C., Umeda H., Nomoto K., Tominaga N., Ohkubo T., 2006, ApJ, 653, 1145

Koeppen J., Arimoto N., 1989, A\&AA, 156, 47

Koeppen J., Arimoto N., 1990, Astron. Astrophys. , 240, 22

Köppen J., Weidner C., Kroupa P., 2007, MNRAS, 375, 673

Kormendy J., 2013, ArXiv e-prints

Kormendy J., Kennicutt Jr. R. C., 2004, araa, 42, 603 
Kroupa P., 2001, MNRAS, 322, 231

Kroupa P., Boily C. M., 2002, MNRAS, 336, 1188

Kroupa P., Weidner C., 2003, ApJ, 598, 1076

Kroupa P., Weidner C., Pflamm-Altenburg J., Thies I., Dabringhausen J., Marks M., Maschberger T., 2013, The Stellar and Sub-Stellar Initial Mass Function of Simple and Composite Populations. Oswalt, T. D. and Gilmore, G., p. 115

Kuntschner H., 2000, MNRAS, 315, 184

La Barbera F., Ferreras I., Vazdekis A., de la Rosa I. G., de Carvalho R. R., Trevisan M., Falcón-Barroso J., Ricciardelli E., 2013, MNRAS, 433, 3017

Lacey C. G., Baugh C. M., Frenk C. S., Silva L., Granato G. L., Bressan A., 2008, MNRAS, 385, 1155

Lada C. J., Lada E. A., 2003, MNRAS, 41, 57

Lagos C. D. P., Cora S. A., Padilla N. D., 2008a, MNRAS, 388, 587

Lagos C. D. P., Cora S. A., Padilla N. D., 2008b, Mon. Not. R. Astron. Soc. , 388, 587

Lagos C. D. P., Padilla N. D., Cora S. A., 2009, MNRAS, 397, L31

Larsen S., 2002, AJ, 124, 1393

Larson R. B., Tinsley B. M., Caldwell C.Ñ., 1980, Astrophys. J. , 237, 692

Li W., Leaman J., Chornock R., Filippenko A. V., Poznanski D., Ganeshalingam M., Wang X., Modjaz M., Jha S., Foley R. J., Smith N., 2011, MNRAS, 412, 1441

Lia C., Portinari L., Carraro G., 2002, MNRAS, 330, 821

López-Corredoira M., Cabrera-Lavers A., Gerhard O. E., 2005, Astron. Astrophys. , 439, 107

Lucatello S., Gratton R. G., Beers T. C., Carretta E., 2005, ApJ, 625, 833

Malbon R. K., Baugh C. M., Frenk C. S., Lacey C. G., 2007, MNRAS, 382, 1394

Marigo P., Girardi L., 2007, A\&A, 469, 239

Marigo P., Girardi L., Bressan A., Groenewegen M. A. T., Silva L., Granato G. L., 2008, A\&A, 482, 883

Marks M., Kroupa P., Dabringhausen J., Pawlowski M. S., 2012, MNRAS, 422, 2246

Matteucci F., 1994, A\&A, 288, 57 
Matteucci F., Brocato E., 1990, Astrophys. J. , 365, 539

Matteucci F., Greggio L., 1986, A\&A, 154, 279

Matteucci F., Romano D., 1999, A\&AR, 265, 311

Mattila S., Dahlen T., Efstathiou A., Kankare E., Melinder J., Alonso-Herrero A., Pérez-Torres M. Á., Ryder S., Väisänen P., Östlin G., 2012, ApJ, 756, 111

McWilliam A., Rich R. M., 1994, Astrophys. J. (Supp.) , 91, 749

McWilliam A., Zoccali M., 2010, Astrophys. J., , 724, 1491

Melinder J., Dahlen T., Mencía Trinchant L., stlin G., Mattila S., Sollerman J., Fransson C., Hayes M., Kankare E., Nasoudi-Shoar S., 2012, A\&A, 545, 96

Menci N., Cavaliere A., Fontana A., Giallongo E., Poli F., Vittorini V., 2004, ApJ, 604, 12

Minniti D., et al. 2010, New Astronomy, 15, 433

Mo H., van den Bosch F. C., White S., 2010, Galaxy Formation and Evolution

Mo H. J., Mao S., White S. D. M., 1998, MNRAS, 295, 319

Mutch S. J., Croton D. J., Poole G. B., 2011, ApJ, 736, 84

Nagashima M., Lacey C. G., Baugh C. M., Frenk C. S., Cole S., 2005, MNRAS, 358, 1247

Nagashima M., Lacey C. G., Okamoto T., Baugh C. M., Frenk C. S., Cole S., 2005, MNRAS, 363, L31

Navarro J. F., Frenk C. S., White S. D. M., 1995, Mon. Not. R. Astron. Soc. , 275, 720

Navarro J. F., Frenk C. S., White S. D. M., 1996, Astrophys. J. , 462, 563

Navarro J. F., Frenk C. S., White S. D. M., 1997, Astrophys. J. , 490, 493

Navarro J. F., Hayashi E., Power C., Jenkins A. R., Frenk C. S., White S. D. M., Springel V., Stadel J., Quinn T. R., 2004, Mon. Not. R. Astron. Soc. , 349, 1039

Neistein E., van den Bosch F. C., Dekel A., 2006, MNRAS, 372, 933

Ness M., Debattista V. P., Bensby T., Feltzing S., Roškar R., Cole D. R., Johnson J. A., Freeman K., 2014, Astrophys. J. , 787, L19

Ness M., Freeman K., Athanassoula E., Wylie-de-Boer E., Bland-Hawthorn J., Asplund M., Lewis G. F., Yong D., Lane R. R., Kiss L. L., 2013, Mon. Not. R. Astron. Soc. , 430, 836 
Ness M., Freeman K., Athanassoula E., Wylie-De-Boer E., Bland-Hawthorn J., Lewis G. F., Yong D., Asplund M., Lane R. R., Kiss L. L., Ibata R., 2012, Astrophys. J. , 756, 22

Nomoto K., Tominaga N., Umeda H., Kobayashi C., Maeda K., 2006, Nuclear Physics A, 777, 424

Ogiya G., Mori M., 2014, Astrophys. J. , 793, 46

Orsi A., Padilla N., Groves B., Cora S., Tecce T., Gargiulo I., Ruiz A., 2014, ArXiv e-prints

Padilla N., Salazar S., Contreras S., Cora S., Ruiz A., 2013, arxiv:1311.3976

Padovani P., Matteucci F., 1993, ApJ, 416, 26

Panter B., Jimenez R., Heavens A. F., Charlot S., 2007, MNRAS, 378, 1550

Parijskij Y.Ñ., 1978, in Longair M. S., Einasto J., eds, Large Scale Structures in the Universe Vol. 79 of IAU Symposium, Search for Primordial Perturbations of the Universe: Observations with RATAN-600 Radio Telescope. p. 315

Pasquini L., Alonso J., Avila G., Barriga P., et al. 2003, in Iye M., Moorwood A. F. M., eds, Instrument Design and Performance for Optical/Infrared Ground-based Telescopes Vol. 4841 of Society of PhotoOptical Instrumentation Engineers (SPIE) Conference Series, Installation and first results of FLAMES, the VLT multifibre facility. pp 1682-1693

Peebles P. J. E., 1969, Astrophys. J. , 155, 393

Peebles P. J. E., 1971, Physical cosmology

Pérez-González P. G., Trujillo I., Barro G., Gallego J., Zamorano J., Conselice C. J., 2008, ApJ, 687, 50

Perlmutter S., Aldering G., Goldhaber G., et al. 1999, Astrophys. J. , 517, 565

Pietrinferni A., Cassisi S., Salaris M., Castelli F., 2004, Astrophys. J. , 612, 168

Pipino A., Devriendt J. E. G., Thomas D., Silk J., Kaviraj S., 2009, A\&A, 505, 1075

Pipino A., Matteucci F., 2004, MNRAS, 347, 968

Planck Collaboration Ade P. A. R., Aghanim N., Armitage-Caplan C., Arnaud M., Ashdown M., AtrioBarandela F., Aumont J., Baccigalupi C., Banday A. J., et al. 2014, Astron. Astrophys. , 571, A16

Recchi S., Calura F., Kroupa P., 2009, A\&A, 499, 711

Renzini A., Buzzoni A., 1986, in Chiosi C., Renzini A., eds, Spectral Evolution of Galaxies Vol. 122 of Astrophysics and Space Science Library, Global properties of stellar populations and the spectral evolution of galaxies. pp 195-231 
Rich R. M., 1988, Astron. J. , 95, 828

Rich R. M., Origlia L., 2005, Astrophys. J. , 634, 1293

Romano D., Karakas A. I., Tosi M., Matteucci F., 2010, A\&A, 522, A32

Ruiz A.Ñ., Cora S. A., Padilla N. D., Domínguez M. J., Tecce T. E., Orsi Á., Yaryura Y. C., García Lambas D., Gargiulo I. D., Muñoz Arancibia A. M., 2014, arxiv:1310.7034

Saglia R. P., Maraston C., Thomas D., Bender R., Colless M., 2002, ApJL, 579, L13

Salpeter E. E., 1955, ApJ, 121, 161

Sánchez-Blázquez P., Gorgas J., Cardiel N., González J. J., 2006, A\&A, 457, 787

Sandage A., Tammann G. A., 1981, A revised Shapley-Ames Catalog of bright galaxies

Sani E., Lutz D., Risaliti G., Netzer H., Gallo L. C., Trakhtenbrot B., Sturm E., Boller T., 2010, MNRAS, 403, 1246

Sarkar P., Yadav J., Pandey B., Bharadwaj S., 2009, Mon. Not. R. Astron. Soc. , 399, L128

Schmidt B. P., Suntzeff N. B., Phillips M. M., Schommer R. A., Clocchiatti A., et al. 1998, Astrophys. J. $, 507,46$

Shadmehri M., 2004, MNRAS, 354, 375

Sheth R. K., Tormen G., 1999, Mon. Not. R. Astron. Soc. , 308, 119

Simien F., de Vaucouleurs G., 1986, Astrophys. J. , 302, 564

Skrutskie M. F., Cutri R. M., Stiening R., Weinberg M. D., et al. 2006, Astron. J. , 131, 1163

Sobral D., Best P.Ñ., Smail I., Mobasher B., Stott J., Nisbet D., 2014, MNRAS, 437, 3516

Sofue Y., Honma M., Omodaka T., 2009, PASJ, 61, 227

Somerville R. S., Hopkins P. F., Cox T. J., Robertson B. E., Hernquist L., 2008, MNRAS, 391, 481

Somerville R. S., Lemson G., Sigad Y., Dekel A., Kauffmann G., White S. D. M., 2001, Mon. Not. R. Astron. Soc. , 320, 289

Somerville R. S., Primack J. R., 1999, Mon. Not. R. Astron. Soc. , 310, 1087

Soto M., Rich R. M., Kuijken K., 2007, Astrophys. J. , 665, L31

Spolaor M., Kobayashi C., Forbes D. A., Couch W. J., Hau G. K. T., 2010, MNRAS, 408, 272 
Springel V., 2005, MNRAS, 364, 1105

Springel V., Wang J., Vogelsberger M., Ludlow A., Jenkins A., Helmi A., Navarro J. F., Frenk C. S.,

White S. D. M., 2008, Mon. Not. R. Astron. Soc. , 391, 1685

Springel V., White S. D. M., Tormen G., Kauffmann G., 2001, MNRAS, 328, 726

Tecce T. E., Cora S. A., Tissera P. B., Abadi M. G., Lagos C. D. P., 2010, MNRAS, 408, 2008

Tegmark M., Strauss M. A., Blanton M. R., Abazajian K., Dodelson S., Sandvik H., Wang X., Weinberg D. H., Zehavi I., Bahcall N. A., et al. 2004, PhyR, 69, 103501

Thies I., Kroupa P., 2007, ApJ, 671, 767

Thies I., Kroupa P., 2008, MNRAS, 390, 1200

Thomas D., 1999, MNRAS, 306, 655

Thomas D., Maraston C., 2003, A\&A, 401, 429

Thomas D., Maraston C., Bender R., Mendes de Oliveira C., 2005, ApJ, 621, 673

Thomas D., Maraston C., Schawinski K., Sarzi M., Silk J., 2010, MNRAS, 404, 1775

Tinsley B. M., 1976, ApJ, 208, 797

Tinsley B. M., 1979, ApJ, 229, 1046

Tortora C., Romanowsky A. J., Napolitano N. R., 2013, ApJ, 765, 8

Trager S. C., Faber S. M., Dressler A., 2008, MNRAS, 386, 715

Trager S. C., Faber S. M., Worthey G., González J. J., 2000, AJ, 119, 1645

Tremaine S., Gunn J. E., 1979, Physical Review Letters, 42, 407

Treu T., Auger M. W., Koopmans L. V. E., Gavazzi R., Marshall P. J., Bolton A. S., 2010, ApJ, 709, 1195

van Dokkum P. G., Conroy C., 2010, NAT, 468, 940

van Dokkum P. G., Conroy C., 2012, ApJ, 760, 70

Vásquez S., Zoccali M., Hill V., Renzini A., González O. A., Gardner E., Debattista V. P., Robin A. C., Rejkuba M., Baffico M., Monelli M., Motta V., Minniti D., 2013, Astron. Astrophys. , 555, A91

Walsh D., Carswell R. F., Weymann R. J., 1979, Nature , 279, 381 
Wang B., Heckman T. M., 1996, Astrophys. J. , 457, 645

Wechsler R. H., Bullock J. S., Primack J. R., Kravtsov A. V., Dekel A., 2002, Astrophys. J., 568, 52

Weidner C., Ferreras I., Vazdekis A., La Barbera F., 2013, MNRAS, 435, 2274

Weidner C., Kroupa P., Larsen S. S., 2004, MNRAS, 350, 1503

Weidner C., Kroupa P., Pflamm-Altenburg J., 2011, MNRAS, 412, 979

Weidner C., Kroupa P., Pflamm-Altenburg J., 2013, MNRAS, 434, 84

Weidner C., Kroupa P., Pflamm-Altenburg J., Vazdekis A., 2013, MNRAS, 436, 3309

White S. D. M., Frenk C. S., 1991, Astrophys. J. , 379, 52

White S. D. M., Frenk C. S., Davis M., 1983, Astrophys. J. , 274, L1

White S. D. M., Frenk C. S., Davis M., Efstathiou G., 1987, Astrophys. J. , 313, 505

White S. D. M., Rees M. J., 1978a, Mon. Not. R. Astron. Soc. , 183, 341

White S. D. M., Rees M. J., 1978b, MNRAS, 183, 341

Woosley S. E., Weaver T. A., 1995, ApJS, 101, 181

Worthey G., Faber S. M., Gonzalez J. J., 1992, ApJ, 398, 69

Worthey G., Tang B., Serven J., 2013, arxiv:1303.2603

Yates R. M., Henriques B., Thomas P. A., Kauffmann G., Johansson J., White S. D. M., 2013, arxiv: 1305.7231

York D. G., Adelman J., Anderson Jr. J. E., Anderson S. F., SDSS Collaboration 2000, Astron. J. , 120, 1579

Zel'dovich Y. B., 1970, Astron. Astrophys. , 5, 84

Zhao D. H., Jing Y. P., Mo H. J., Borner G., 2003, Astrophys. J. , 597, L9

Zoccali M., Hill V., Lecureur A., Barbuy B., Renzini A., Minniti D., Gómez A., Ortolani S., 2008, A\&A, 486, 177

Zoccali M., Lecureur A., Barbuy B., Hill V., Renzini A., Minniti D., Momany Y., Gómez A., Ortolani S., 2006, Astron. Astrophys. , 457, L1 
Zoccali M., Renzini A., Ortolani S., Greggio L., Saviane I., Cassisi S., Rejkuba M., Barbuy B., Rich R. M., Bica E., 2003, Astron. Astrophys. , 399, 931

Zwicky F., 1933, Helvetica Physica Acta, 6, 110

Zwicky F., 1937a, Physical Review

Zwicky F., 1937b, Astrophys. J., 86, 217 
